\title{
Nutrition care and its education
}

\author{
Citation for published version (APA):
}

Mogre, V. (2018). Nutrition care and its education: medical students' and doctors' perspectives. [Doctoral Thesis, Maastricht University]. Datawyse / Universitaire Pers Maastricht. https://doi.org/10.26481/dis.20181107vm

Document status and date:

Published: 01/01/2018

DOI:

10.26481/dis.20181107vm

Document Version:

Publisher's PDF, also known as Version of record

\section{Please check the document version of this publication:}

- A submitted manuscript is the version of the article upon submission and before peer-review. There can be important differences between the submitted version and the official published version of record.

People interested in the research are advised to contact the author for the final version of the publication, or visit the DOI to the publisher's website.

- The final author version and the galley proof are versions of the publication after peer review.

- The final published version features the final layout of the paper including the volume, issue and page numbers.

Link to publication

\footnotetext{
General rights rights.

- You may freely distribute the URL identifying the publication in the public portal. please follow below link for the End User Agreement:

www.umlib.nl/taverne-license

Take down policy

If you believe that this document breaches copyright please contact us at:

repository@maastrichtuniversity.nl

providing details and we will investigate your claim.
}

Copyright and moral rights for the publications made accessible in the public portal are retained by the authors and/or other copyright owners and it is a condition of accessing publications that users recognise and abide by the legal requirements associated with these

- Users may download and print one copy of any publication from the public portal for the purpose of private study or research.

- You may not further distribute the material or use it for any profit-making activity or commercial gain

If the publication is distributed under the terms of Article $25 \mathrm{fa}$ of the Dutch Copyright Act, indicated by the "Taverne" license above, 


\section{Nutrition care and}

its education:

medical students' and doctors' perspectives

Victor Mogre 
The research reported here was carried out at:

DI Maastricht University in Learning!

In the School of Health Professions Education

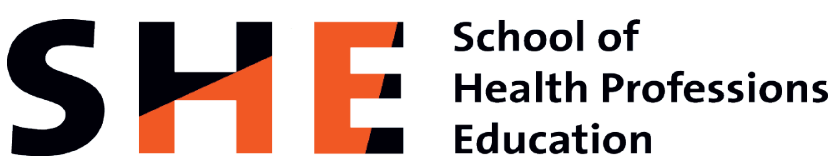

(C) copyright Victor Mogre, Maastricht 2018

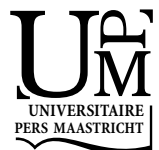

ISBN 9789463800068 


\title{
Nutrition care and its education: medical students' and doctors' perspectives
}

\author{
DISSERTATION \\ to obtain the degree of Doctor at Maastricht University, \\ on the authority of the Rector Magnificus, Prof. Dr. Rianne M. Letschert \\ in accordance with the decision of the Board of Deans, \\ to be defended in public \\ on Wednesday, $7^{\text {th }}$ November 2018 at 10.00 hours
}

by

Victor Mogre 


\section{Supervisor}

Prof. dr. A.J.J.A. Scherpbier

\section{Co-supervisors}

Dr. F.C.J. Stevens

Dr. P. A. Aryee, University for Development Studies, Tamale, Ghana

\section{Assessment Committee}

Prof. dr. W.N.K.A van Mook (Chairman)

Prof. dr. G. Croiset, UMCG, Groningen

Prof. dr. A.D.C. Jaarsma, UMCG, Groningen

Prof. dr. S.P.J. Kremers

Dr. J. de Nooijer 


\section{Contents}

Chapter 1 General Introduction

Chapter 2 Medical students' perspectives on health professionals'

responsibility regarding nutrition care and why doctors should

learn about nutrition: A qualitative study

(Under review) Education for Health

Chapter 3 Nutrition in medicine: Medical students' satisfaction, perceived relevance and preparedness for practice Health Professions Education 2018; 4(1):31-38. DOI:

https://doi.org/10.1016/j.hpe.2017. 02.003

Chapter 4 Future doctors' nutrition-related knowledge, attitudes and selfefficacy regarding nutrition care in the general practice setting: A cross-sectional survey Medical Science Educator 2017; 27(3):481-488.

DOI: 10.1007/s40670-017-0413-5

Chapter 5 Nutrition care practices, barriers, competencies and education in nutrition: A survey among Ghanaian medical doctors Medical Science Educator 2018; 1-10. DOI: https://doi.org/10.1007/s40670-018-0591-9

Chapter 6 Why nutrition education is inadequate in the medical curriculum:

A qualitative study of students' perspectives on barriers and strategies

BMC Medical Education 2018; 18(1):26. DOI: 10.1186/ s12909-018-1130-5.

Chapter 7 PROTOCOL: A realist review of educational interventions to improve the delivery of nutrition care by doctors and future doctors Systematic Reviews 2014; 3:148. DOI: 10.1186/2046-4053-3-148

Chapter 8 Realist synthesis of educational interventions to improve nutrition care competencies and delivery by doctors and other healthcare professionals

BMJ Open 2016; 6(10): e01008. DOI: 10.1136/bmjopen-2015-010084. 115

Chapter 9 General Discussion 
Summary

Samenvatting

Valorisation

205

Acknowledgement

213

Curriculum Vitae

215

List of Publications

217 
Chapter

General Introduction 



\section{Introduction and scope of the thesis}

This chapter provides a background of the role of nutrition in health and illness, and of nutrition as a significant contributor to morbidity and mortality in the world, sub-Saharan Africa and specifically Ghana. The chapter continues with a description of the nutrition care process and associated competencies. Further on in the chapter we provide a justification of why it is important for healthcare professionals, especially doctors, to provide nutrition care. Then we describe nutrition education in the medical curriculum. We conclude the chapter by stating our research questions and providing an overview of the research projects included in the thesis.

\section{Background}

Translating into the loss of 3 million young lives every year, under nutrition is the cause of about $50 \%$ of all deaths in children under $5^{1}$. In the first 1,000 days of a child's life, under nutrition can cause irreversible stunted growth, impaired cognitive ability and poor school and work performance ${ }^{2}$. Under nutrition also makes a child susceptible to common infections, increases the frequency and severity of such infections and prolongs recovery from such infections. Apart from Asia, Africa bears the greatest burden of all forms of malnutrition in the world. According to the UNICEF/WHO/World Bank Group Joint Child Malnutrition Estimates for 2017, 59 million children are stunted (i.e. children's height is low for their age); 14 million are wasted (i.e. children's weight is low for their height) and 10 million are overweight ${ }^{1}$. According to the 2014 Ghana Demographic and Health Survey (GDHS) findings on the nutritional status of Ghanaian children, $19 \%$ were stunted; $5 \%$ were wasted and $11 \%$ were underweight (i.e. children's weight is low for their age) ${ }^{3}$. With one-third and $82 \%$ of its children being stunted and anaemic respectively, the Northern part of Ghana (the setting of this thesis) has the highest prevalence of child under nutrition ${ }^{3}$.

While under nutrition and infectious diseases affect a large proportion of children in sub-Saharan Africa, adults are hit with rising prevalence of non-communicable diseases (NCDs), such as obesity, diabetes, cardiovascular diseases, cancers, etc. NCDs are estimated to be the leading cause of mortality in sub-Saharan Africa by the year $2050^{4}$. Currently, the sub-region has the highest prevalence of hypertension, a precursor of stroke, and other cardiovascular diseases.

The management of these conditions at both clinical and public health levels require nutrition care. The role of nutrition in health promotion, disease prevention and treatment of chronic disease has also been well documented ${ }^{5,6}$. It has been shown that nutrition interventions can decrease morbidity, mortality, human suffering, and medical costs ${ }^{7-10}$. In comparison with pharmacological interventions to patients, there is increasing evidence that the benefits of nutrition, diet and physical activity interventions are either 
comparable or better, with attended benefits of low side effects, reduced risk and lower cost ${ }^{11-13}$. Nutrition care may thus be more appropriate and cost effective than other treatment options (e.g., pharmacological intervention) for the challenged healthcare systems of Ghana and other sub-Saharan Africa countries.

\section{Concept of nutrition care and competency}

Nutrition care is the application of nutrition principles to the prevention and treatment of patients' conditions by health care providers ${ }^{14}$. Depending on the condition of the patient, it could include nutrition screening/assessment (e.g., food/nutrition history, biochemical data, medical tests and procedures, body mass index, etc.), nutrition diagnosis, nutrition intervention (education and counselling), nutrition monitoring and evaluation. Nutrition care competency is a broad concept that encompasses knowledge, skills, selfefficacy, interest and attitudes. It is the capability to apply or use nutrition knowledge and skills/abilities needed to successfully perform "critical work functions" or tasks (delivering nutrition care to patients) in a defined workplace (e.g., at the hospital). The delivery of nutrition care by healthcare providers, and for the purposes of this study the medical doctor, is a capability that is influenced by cognitive and affective processes of the individual as well as the social, cultural and physical environmental context in which the behaviour is executed. The nutrition care process is considered as a cyclic process ${ }^{15}$ (See Figure 1). It consists of four distinct, but interrelated and connected steps: nutrition assessment, nutrition diagnosis, nutrition intervention and nutrition monitoring and evaluation ${ }^{15}$. The nutrition care process also has applicability in both individuals and groups, in community-based programmes aimed at promoting health and disease prevention 15. It is important to note that the role of the doctor is very critical in each of the steps of the nutrition care process. More so, the healthcare process requires a multidisciplinary approach, and every provider should be clearly aware of the role of the other team member. Therefore, nutrition care in the context of this thesis refers to not only taking a nutrition history, but also providing dietary counselling, health education, and referring patients to qualified dieticians and/or nutrition specialists ${ }^{16}$. 


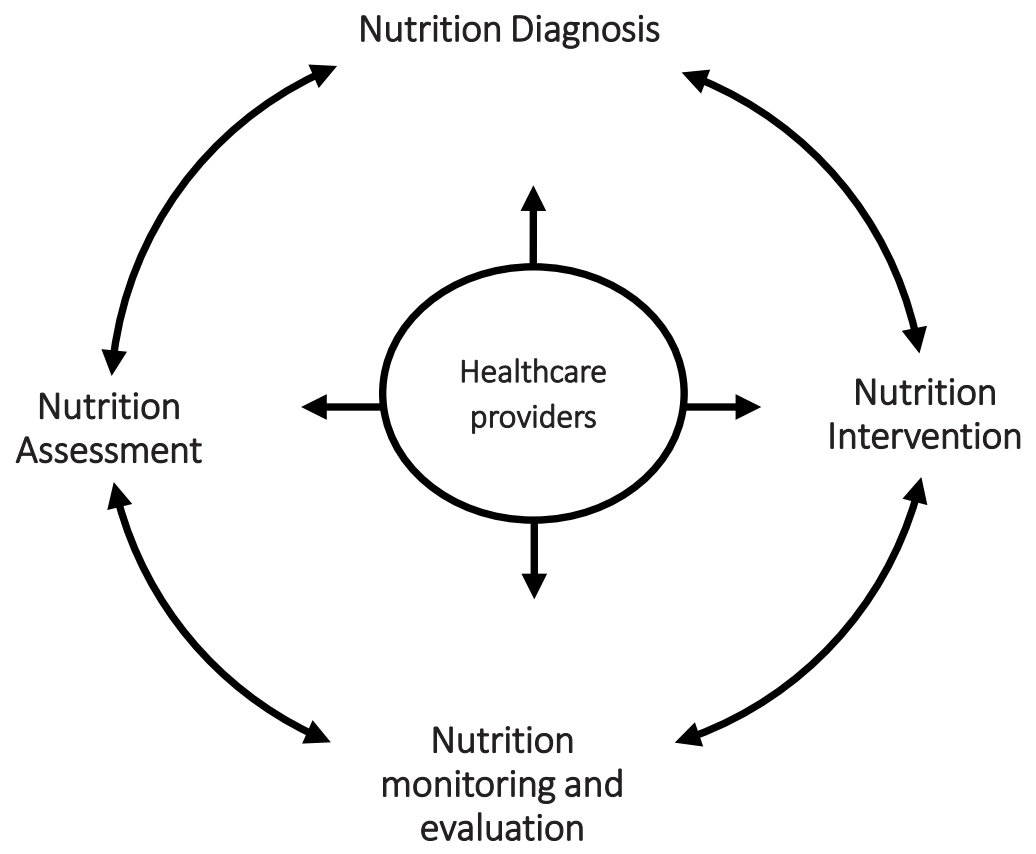

Figure 1: The Nutrition care model

\section{Why should doctors provide nutrition care?}

There is consensus among healthcare professionals, including doctors that nutrition care is central to healthcare and also important to their practice ${ }^{17}$.

In recent times, an increasing number of individuals are becoming more concerned and conscious of what they eat, and will seek for nutrition advice and care from doctors and other healthcare providers. Making it possible for individuals to seek for nutrition information and advice from doctors and other healthcare providers may discourage them from seeking such information from potentially unsubstantiated sources that may not be supported by evidence ${ }^{18-20}$. This may result in helping to reduce nutrition fraud and food faddism ${ }^{17}$. The WHO also recommends that medical professionals should be supported to take an active role in helping individuals to adopt healthy dietary habits ${ }^{21}$.

In developing countries like Ghana, doctors drive the healthcare system and are considered as important and credible sources of nutrition information. It is thus recognized that doctors play an important role in providing nutrition care. The big question, however, is whether they are adequately prepared to provide such care. 


\section{Nutrition education in medical training}

Nutrition education may not convey the same meaning to all nutrition and health professionals $^{22}$. However, two distinctions can be made: (1) education about nutrition and (2) education in nutrition for action that focuses on practices. Using the definition provided by the American Dietetic Association, nutrition education refers to the "learning experiences designed to facilitate the voluntary adoption of eating and other nutrition-related behaviours conducive to health and well-being" ${ }^{23}$. Focusing on individuals, their lifestyles, motivations and social context, this approach has been designated as action oriented. This form of nutrition education has several names such as health promotion, awarenessraising, social marketing, information, education and communication (IEC), communication for behaviour change $(\mathrm{CBC})$, demonstration, behaviour change communication (BCC), among others ${ }^{22}$. Nutrition education can also be used to describe professional nutrition training program to equip healthcare professionals with nutrition competencies to enable them to provide nutrition care which may include nutrition education. This form of nutrition education is commonly used by the US government and universities to describe professional courses in nutrition and has been adopted in several publications ${ }^{22}$. Although this form of nutrition education may result in helping health professionals to adopt healthy dietary habits, the terminology nutrition education, as adopted in this thesis, is aligned with the deliberate inclusion of nutrition syllabi or topics into the medical curriculum, with the aim of helping medical students to acquire nutrition-related competencies and skills and to affect nutrition practice behaviour. It also includes continuing education programmes/interventions that are aimed at improving the nutrition practice behaviour of medical doctors. The two differing definitions of nutrition education outlined above are interrelated in that one is a precursor of the other. The doctor requires nutrition education during his/her training in order to help the patient adopt and practice healthy nutrition and dietary habits.

Undergraduate medical education lays the foundation for future medical practice and over the years nutrition has been inadequately represented in many medical schools in the world ${ }^{24}$. In the U.S. for instance, a 1985 study from the National Academy of Sciences on Nutrition Education of Medical Schools reported that only 21 hours of required nutrition instruction was provided for an entire four-year undergraduate medical education ${ }^{25}$. The report found this to be woefully inadequate and recommended a minimum of 25 hours of nutrition instruction to adequately trained medical students to meet the nutrition care needs of their patients. Although the 1985 report resulted in several professional groups issuing statements, publications and analyses as well as a congress issuing a mandate for improved nutrition education ${ }^{24-26}$, medical schools have not met the requirement, and a downward trend in the number of hours of required nutrition instruction is still ubiquitous in the US ${ }^{24,27}$. Publications from other parts of the world, such as 
from the UK, Australia, Canada, and New Zealand, also report doctors' receipt of inadequate nutrition education during medical school. Both medical students and doctors alike report receiving little or no formal nutrition education in medical school.

Inadequate nutrition education may partly be due to barriers such as: low priority for nutrition training, the curriculum emphasizing disease treatment but not prevention, lack of physician nutrition specialists to provide nutrition education to medical students, and resistance to the inclusion of new courses or lectures to the curriculum ${ }^{28-31}$. In addition, many medical schools do not have separate departments for nutrition to employ faculty that could provide nutrition education to medical students. Inadequate nutrition education during undergraduate medical education has led to many doctors frequently reporting their inability to provide the needed nutrition care to their patients. Studies that have assessed the nutrition practice behaviour of doctors have found them to recognize the importance of nutrition in patient care, but they do not feel confident, self-efficacious or adequately prepared to provide nutrition care to their patients ${ }^{32-37}$. Doctors also report inadequate nutrition-related knowledge and may sometimes have less nutrition knowledge than their patients ${ }^{24,38}$. This is substantiated by doctors routinely reporting that they would benefit from further training in a number of nutrition topics ${ }^{39-41}$. Several studies have also found doctors reporting low self-efficacy in the provision of nutrition care to patients ${ }^{37,42}$. If doctors do not feel competent or comfortable to provide nutrition care, patients may not receive the nutrition care they may require.

\section{Problem statement and research questions}

With regard to the peculiar manpower and resource-limited situation of sub-Saharan Africa, including countries like Ghana, practicing physicians (including specialists/consultants), medical undergraduates/officers and senior medical students provide health care to the general population. The current literature regarding nutrition education and the nutrition practice behaviour of medical students and doctors originate from Western countries, such as the USA, Canada, Australia, and the UK, among others. Studies from sub-Saharan Africa, such as Ghana, are limited. Taking note of the peculiarity of the healthcare system and the medical education environment of the sub-region, it is imperative to undertake a needs assessment of the situation of medical nutrition education using Ghana as a case study. This research serves as a first step towards providing information on nutrition education, nutrition care competency and practice behaviour in subSaharan Africa. It also adds to the already existing literature of nutrition education and nutrition care practice behaviour and provides evidence on how educational interventions could be developed to improve nutrition practice behaviour. Thus, the general research question of this thesis is:

What is the current practice of a) nutrition education for medical students and b) nutrition care of practicing doctors in Ghana? 
In order to answer this question, the following specific research questions were investigated as part of the thesis:

1. How do medical students perceive the role of nutrition care in their training, and what do they perceive to be the roles and responsibilities of doctors in the provision of nutrition care? (Chapters 2 \& 6 )

2. What are the nutrition care competence and self-efficacy of medical students and practicing doctors? (Chapters 3, 4, 5 \& 6)

3. What are practicing doctors' nutrition care practices and referral behaviours in the general practice setting, and what are the barriers to the provision of nutrition care? (Chapters 5 \& 6)

4. What are the barriers to effective nutrition education and how could nutrition education be improved in the medical curriculum? (Chapters 3 and 6)

5. How do practicing doctors perceive the effectiveness of the nutrition-related training and learning resources that they are currently using and may use in the future? (Chapter 5)

6. How to design a protocol to undertake a realist review of educational interventions to improve nutrition care competence and delivery? (Chapter 7)

7. How, for whom, why and in what circumstances do educational interventions work to improve nutrition care competence and nutrition practice behaviour among doctors and other healthcare professionals? (Chapter 8)

\section{Studies addressing the research questions}

The answers to the research questions have been contributed in diverse ways by the chapters of this thesis.

Chapter 2 is a qualitative study of senior medical students' perception of why medical students should learn about nutrition and why doctors should provide nutrition care as part of their daily practice. Having considered students' views of why doctors should provide nutrition care, chapters 3, 4, 5 and 6 focuses on a needs assessment of the situation of nutrition education, nutrition care competencies and barriers to effective nutrition education.

Chapter 3 is a cross-sectional study of students' perception of the adequacy of and satisfaction with nutrition education as well as their preparedness to provide nutrition care.

Chapter 4 is another cross-sectional study that considers students' nutrition care competency levels such as knowledge, attitude and self-efficacy.

Another cross-sectional study, chapter 5 presents the nutrition care competency levels of practicing medical doctors, their nutrition practice behaviour as well as their perception of the adequacy and satisfaction with their nutrition education during medical 
school. Doctors' perception of the barriers to the provision of nutrition care is also presented. Finally, the chapter provides data on practicing doctors' perception of nutritionrelated training and learning resources that could be effective in continuing education in nutrition during practice.

Chapter 6 is a qualitative study that presents students' views of why nutrition education is inadequate, and strategies that could be implemented to improve nutrition education. Again, students' perception of the roles and responsibilities of doctors regarding nutrition care is also presented.

Chapters 7 and 8 are closely related as they focus on a realist review of educational interventions to improve the nutrition care competency and practice behaviour of doctors and other healthcare professionals.

Chapter 9 draws conclusions, discussions and implications of the results of the previous chapters for improving nutrition education and nutrition practice behaviour among medical doctors. 


\section{References}

1. UNICEF, WHO and World Bank Group. Levels and Trends in Child Malnutrition. UNICEF/WHO/World Bank Group Joint Child Malnutrition Estimates Key Findings of the 2016 edition. New York: UNICEF; Geneva: WHO; Washington DC: World Bank 2017.

2. UNICEF. Undernutrition contributes to nearly half of all deaths in children under 5 and is widespread in Asia and Africa. 2017. http://data.unicef.org/nutrition/malnutrition.html. Accessed 20/11/2017, 2017.

3. Ghana Statistical Service, Ghana Health Service, ICF International. Ghana Demographic and Health Survey 2014. Ghana Demographic and Health Survey 2014. Rockville, Maryland, USA: GSS, GHS, and ICF International. 2015.

4. World Health Organization. New data highlight increases in hypertension, diabetes incidence. 2012; http://www.who.int/mediacentre/news/releases/2012/world_health_statistics_20120516/en/.

5. Hu SP, Wu MY \& Liu JF. Nutrition knowledge, attitude and practice among primary care physicians in Taiwan. J Am College Nutr 1997;16(5):439-442.

6. Schaller C, James EL. The nutritional knowledge of Australian nurses. Nurse Educ Today 2005;25(5):4054412.

7. Gibbs J, Cull W, Henderson W, Daley J, Hur K, Khuri SF. Preoperative serum albumin level as a predictor of operative mortality and morbidity: results from the National VA Surgical Risk Study. Arch Surg 1999;134(1):36-42.

8. Montgomery DL, Splett PL. Economic benefit of breast-feeding infants enrolled in WIC. J Am Diet Assoc 1997;97(4):379-385.

9. van Weel C. Morbidity in family medicine: the potential for individual nutritional counseling, an analysis from the Nijmegen Continuous Morbidity Registration. Am J Clin Nutr 1997;65(6 Suppl):1928S-1932S.

10. Halsted CH. Clinical nutrition education--relevance and role models. Am J Clin Nutr 1998;67(2):192-196.

11. Estruch R, Ros E, Salas-Salvadó J, et al. Primary prevention of cardiovascular disease with a Mediterranean diet. N Engl J Med 2013;368(14):1279-1290.

12. Sacks FM, Svetkey LP, Vollmer WM, et al. Effects on blood pressure of reduced dietary sodium and the Dietary Approaches to Stop Hypertension (DASH) diet. N Engl J Med 2001;344(1):3-10.

13. Look AHEAD Research Group. Cardiovascular effects of intensive lifestyle intervention in type 2 diabetes. N Engl J Med 2013;2013(369):145-154.

14. Young EA, Weser E, McBride H, Page C, Littlefield J. Development of core competencies in clinical nutrition. Am J Clin Nutr 1983;38(5):800-810.

15. Lacey K, Pritchett E. Nutrition care process and model: ADA adopts road map to quality care and outcomes management. J Am Diet Assoc 2003;103(8):1061-1072.

16. Pearson TA, Bloch R, Dorantes J, et al. The rationale for renewed interest by physicians in nutrition. Curr Atheroscler Rep 1999;1(3):173-175.

17. Schulman J. Nutrition education in medical schools: trends and implications for health educators. Med Educ Online 1999;4(1).

18. American Dietetic Association. Position of The American Dietetic Association: Nutrition-an essential component of medical education. J Am Diet Assoc 1994;94(5):555-557.

19. Eisenberg DM, Kessler RC, Foster C, Norlock FE, Calkins DR, Delbanco TL. Unconventional medicine in the United States--prevalence, costs, and patterns of use. N Engl J Med 1993;328(4):246-252.

20. McLaren DS. Nutrition in medical schools: a case of mistaken identity. Am J Clin Nutr 1994;59(5):960.

21. World Health Organization. WHO Technical Report Series-Diet, nutrition and the prevention of chronic diseases. WHO 2003. Geneva.

22. McNulty J. Challenges and issues in nutrition education. Nutrition Education and Consumer Awareness Group, Food and Agriculture Organization of the United Nations 2013. Rome

23. American Dietetic Association. International Dietetics and Nutritional Terminology - Reference Manual: Standard Language for the Nutrition Care Process. 3rd Edition. Chicago, IL: American Dietetic Association 2011. 
24. Adams KM, Kohlmeier M, Powell M, Zeisel SH. Nutrition in medicine: nutrition education for medical students and residents. Nutr Clin Prac 2010;25(5):471-480.

25. National Research Council. Nutrition education in US medical schools. Washington, DC: National Academies Press 1985.

26. Davis $\mathrm{CH}$. The report to Congress on the appropriate federal role in assuring access by medical students, residents, and practicing physicians to adequate training in nutrition. Public Health Rep. 1994;109(6):824.

27. Adams KM, Butsch WS, Kohlmeier M. The state of nutrition education at US medical schools. J Biomed Educ 2015;2015.

28. Walker WA. Innovative teaching strategies for training physicians in clinical nutrition: an overview. J Nutr 2003;133(2):541S-543S.

29. Makowske M, Feinman RD. Nutrition education: a questionnaire for assessment and teaching. Nutr J 2005;4(1):2.

30. Touger-Decker R. Nutrition education of medical and dental students: innovation through curriculum integration. Am J Clin Nutr 2004;79(2):198-203.

31. Wong V, Millen BE, Geller AC, Rogers AE, Maury J-J, Prout MN. What's in store for medical students? Awareness and utilization of expert nutrition guidelines among medical school preceptors. Prev Med 2004;39(4):753-759.

32. Temple NJ. Survey of nutrition knowledge of Canadian physicians. J Am Coll Nutr 1999;18(1):26-29.

33. Weinsier RL, Boker JR, Morgan SL, et al. Cross-sectional study of nutrition knowledge and attitudes of medical students at three points in their medical training at 11 southeastern medical schools. Am J Clin Nutr 1988;48(1):1-6.

34. Levine BS, Wigren MM, Chapman DS, Kerner J, Bergman R, Rivlin R. A national survey of attitudes and practices of primary-care physicians relating to nutrition: strategies for enhancing the use of clinical nutrition in medical practice. Am J Clin Nutr 1993;57(2):115-119.

35. Soltesz KS, Price JH, Johnson LW, Tellijohann SK. Family physicians' views of the preventive services task force recommendations regarding nutritional counseling. Arch Fam Med 1995;4(7):589.

36. Cimino JA. Why can't we educate doctors to practice preventive medicine? Prev Med 1996;25(1):63-65.

37. Kushner RF. Barriers to providing nutrition counseling by physicians: a survey of primary care practitioners. Prev Med 1995;24(6):546-552.

38. Lazarus K. Nutrition practices of family physicians after education by a physician nutrition specialist. Am J Clin Nutr 1997;65(6):2007S-2009S.

39. Coleman VH, Laube DW, Hale RW, Williams SB, Power ML, Schulkin J. Obstetrician-gynecologists and primary care: training during obstetrics-gynecology residency and current practice patterns. Acad Med 2007;82(6):602-607.

40. Mihalynuk TV, Knopp RH, Scott CS, Coombs JB. Physician informational needs in providing nutritional guidance to patients. Fam Med 2004;36(10):722-726.

41. Roberts KB, Starr S, DeWitt TG. The University of Massachusetts Medical Center office-based continuity experience: are we preparing pediatrics residents for primary care practice? Pediatrics. 1997;100(4):e2e2.

42. Vetter ML, Herring SJ, Sood M, Shah NR, Kalet AL. What do resident physicians know about nutrition? An evaluation of attitudes, self-perceived proficiency and knowledge. J Am Coll Nutr 2008;27(2):287-298. 



\section{Chapter}

\section{Medical students' perspectives on health professionals' responsibility regarding nutrition care and why doctors should learn about nutrition: A qualitative study}

Victor Mogre, Fred C.J. Stevens, Paul A. Aryee, Anthony Amalba, and Albert J.J.A Scherpbier 


\section{Abstract}

Background: Improved dietary and nutrition behaviour may help reduce the occurrence of non-communicable diseases which have become global public health emergencies in recent times. However, doctors do not readily provide nutrition counselling to their patients. We explored medical students' perspectives on health professional's nutrition care responsibility, and why doctors should learn about nutrition and provide nutrition care in the general practice setting.

Methods: Semi-structured interviews were conducted among 23 undergraduate clinical level medical students (referred to as medical students). All interviews were recorded and transcribed verbatim with data analysis following a comparative, coding and thematic process.

Results: Medical students were of the view that all health professionals who come into contact with patients in the general practice setting are responsible for the provision of nutrition care to patients. Next to nutritionists/dieticians, medical students felt doctors should be more concerned with the nutrition of their patients than any other health care professional in the general practice setting. Reasons why doctors should be more concerned about nutrition were: patients having regular contacts with the doctor; doctors being the first point of contact; patients having more trust in the doctors' advice; helping to meet the holistic approach to patient care and the fact that nutrition plays an important role in health outcomes of the patient.

Discussion: Medical students perceived all health professionals to be responsible for nutrition care and underscored the need for doctors to learn about nutrition and to be concerned about the nutrition of their patients.

Key words: Nutrition education, Medical students, Nutrition care, General practice setting, Doctors 


\section{Background}

Poor nutrition is a key risk factor implicated in the pathogenesis of several diseases of global public health importance including obesity, diabetes, cancer, stroke, hypertension among others ${ }^{1}$. The WHO estimates these non-communicable diseases (NCDs) as among the leading causes of mortality globally, accounting for $60 \%$ of all deaths ${ }^{2}$.

Improving the nutrition of individuals is very important and a major step in the prevention and management of these NCDs. Doctors serve as trusted and reliable sources of nutrition and health information ${ }^{3}$ and have the opportunity to play a significant role in the nutrition care and wellbeing of their patients.

However, doctors do not frequently provide such nutrition care to their patients partly due to inadequate nutrition education during medical training, lack of time ${ }^{4,5}$ and a number of other barriers. Medical students also frequently report inadequate nutrition education.

Although it is generally accepted that nutrition is important to healthcare, studies exploring medical students' perspectives of why they (students) should learn about nutrition, and as doctors should provide nutrition care are lacking. It is imperative to explore medical students' views on these in order to help them know their limits regarding nutrition, helping them to recognise the multidisciplinary nature of nutrition care and providing opportunities to gauge their acceptability or attitudes regarding the provision of nutrition care during practice. We thus explored medical students' perspectives of which healthcare professional is responsible for the provision of nutrition care and why doctors should learn about nutrition and provide nutrition care to patients in the general practice setting?

\section{Methods}

We recruited participants from the University for Development Studies, School of Medicine and Health Sciences (UDS-SMHS) using a purposive sampling approach. Our participants included only clinical level medical students (referred to as medical students). All participants were contacted by VM through face-to-face meetings. The purpose of the research was explained to the students and informed consent was sought during such meetings. The research was approved by the Navrongo Health Research Institutional Review Board (Ethics Approval ID: NHRCIRB 209). All data was collected using semi-structured interviews informed by open ended questions that explored participants' values, attitudes, experiences, and opinions. All interviews were transcribed verbatim, without identifying information and transcriptions were entered into MAXQDA version 12 , a qualitative data analysis software. Subsequent data analyses were conducted using a comparative strategy. 


\section{Results}

From the 23 students who agreed to participate, $61 \%$ were male students, $39 \%(n=9)$ in clinical year 2 and 30\% (n=7) each in clinical years 1 and 3.Two major themes emerged from the data: whose responsibility it is to provide nutrition care and why doctors should learn and provide nutrition care in the general practice setting.

Whose responsibility is it to provide nutrition care in the general practice setting?

Generally, medical students' opined that all health professionals (i.e. doctors, nutritionists, dieticians, nurses, physician assistants, nurse practitioners, etc) who come into contact with patients in the general practice setting have a role to play and should be responsible for the provision of nutrition care to patients.

"I think all health professionals should give nutritional care to patients including doctors in particular, nutritionist and any other health provider within the hospital should have the knowledge of nutrition and be capable of assisting as the need arises."

In terms of ranking, medical students rated doctors next to nutritionists and dieticians to be more concerned in the provision of nutrition care to patients.

Why should doctors learn about nutrition and provide nutrition care to patients in the general practice setting?

Medical students had the opinion that because doctors have regular/frequent contacts with patients and usually has a good overview of the patient's medical history, it is important they play an important and active role in the nutrition care of the patient.

"The doctors treat the patients directly with the diseases they present to them in the consulting room. So it's also important that the doctor who knows the kind of diseases that the patient is having can also advise the patient on the type of food they should eat. After that the patient can go and see the nutritionists. But the doctor can also play a major role in advising the patient on what to take because they are taking care of them on a daily or monthly basis."

Some medical students had the opinion that doctors are the first point of contact and should be involved in providing nutrition care such as diagnosing and referring patients to nutritionists and dieticians. They are the gate keepers of the healthcare system and determine the kind of treatment needed for every patient. And as such should have sufficient nutrition-related competencies. 
"Doctors have a huge role because usually at the OPD they see the patient first and some of them do not make it to the specialist to get that information. So I think if the doctors are adequately versed with nutrition information, I think they can do more work as in educating the patients about nutrition."

Furthermore, medical students opined that patients have trust in the advice of doctors and are more likely to adhere to the doctor's advice. This is illuminated by a typical experience of a student below.

"What I realize is that patients tend to listen to what doctors say much more. Even more than the nutritionist! So when you refer them there, they go alright. But they come back and they are like wanting you to see 'oh this is what he has done'. They want you to approve of it as to whether 'is it the right thing?' should they go on with it?"

Interestingly, one student opined that providing nutrition care to patients will also help to build and maintain patients' trust in the doctor.

"Yes they should. As I said we are the first point of call so if a patient comes and you have to refer the patient he or she loses some sort of trust in you. He or she knows that you are the one to take care of him."

Also medical students recognized that in providing nutrition care to a patient a holistic, multidisciplinary approach is required and the doctor, an important stakeholder should be involved in the process.

"Doctors should provide nutrition care. In managing the patient, we believe in the synergistic approach. All hands on deck. So doctors should put a hand in it and should not be like this is a nutritionist job or this is not a doctor's job."

Some medical students also had the opinion that the mere fact that nutrition plays an important role in the treatment outcomes of the patient, it is only ordinary and important for the doctor to be concerned with the nutrition needs of the patient.

"Because in the management of certain conditions, if the nutrition is not taken into consideration, whatever treatment you might be giving won't help. So it is very important". "With medicine, I think nutrition should be a major component because most of the diseases especially here in Ghana are connected to nutritional status."

Furthermore, medical students reported that since doctors are responsible for all aspects of care of the patient, nothing prevents them from providing nutrition care which in their opinion, is also part of routine care.

"The other reason is that as a physician you will be managing the patient on all aspects of their health not just a disease condition. Because we know how nutrition plays in disease. You need all that information that's why I think physicians should be involved." 


\section{Discussion}

It is quite heartening that medical students in this study have a perception that all health professionals who come into contact with patients in the general practice setting should be concerned about the nutrition care of their patients. Furthermore, their recognition of the fact that nutritionists and dieticians have a more and specialized role to play in the provision of nutrition care to patients deserves commendation. These views show that they recognize the multidisciplinary nature of nutrition care and value the specialized role of the nutritionists/dietician ${ }^{6}$.

The fact that medical students felt that doctors (except for nutritionists and dieticians) should be more interested in the nutrition of their patients, demonstrates a positive attitude of these medical students to the provision of nutrition care. Students' perspectives that doctors see patients more regularly and are the first point of contact in the general practice setting and as a result should provide nutrition care is consistent with previous reports ${ }^{7,8}$.

In accordance with previous studies, students opined that patients consider doctors to be credible sources of nutrition information and prefer to receive nutrition advice from doctors $^{9}$

Medical students' recognition of nutrition care as being an opportunity to ensure holistic care and inter-professional collaboration for patient care is rather stimulating as the general practice setting presents a good opportunity to provide preventive care ${ }^{9,10}$. Our study is not without limitations. Recruitment of students from a single medical school may limit the generalizability of our findings. The findings of this study serve as a proxy for medical students' readiness to embrace educational interventions to improve nutrition education and to provide nutrition care when they become medical doctors.

\section{Acknowledgements}

Authors wish to thank all students who agreed and consented to participate in the study.

\section{Funding}

This research did not receive any specific grant from funding agencies in the public, commercial, or non-for-profit sectors.

\section{Competing interests}

None declared 


\section{Ethical approval}

Ethical approval for this study was granted by the Navrongo Health Research Centre Institutional Review Board (NHRCIRB) (Ethics Approval ID: NHRCIRB209), Ghana. Students gave a written consent prior to participating in the study. The data was handled in anonymously and confidentially.

\section{Authors' contribution}

VM conceived and designed the study; collected and performed data analysis and interpretation, and drafting of the manuscript. FCJS, PAA, and AJJAS jointly undertook critical revision of the manuscript. All authors approved the manuscript for publication. 


\section{References}

1. Bipartisan Policy Center. Teaching nutrition and physical activity in medical school: training doctors for prevention oriented care: Bipartisan Policy Center, Washington, DC; 2014.

2. World Health Organization. Global strategy on diet, physical activity and health. Geneva 2004.

3. Abramson S, Stein J, Schaufele M, Frates E, Rogan S. Personal exercise habits and counseling practices of primary care physicians: a national survey. Clin J Sport Med 2000;10(1):40-48.

4. Kolasa KM, Rickett K. Barriers to providing nutrition counseling cited by physicians: a survey of primary care practitioners. Nutr Clin Pract 2010;25(5):502-509.

5. Crowley J, Ball L, Han DY, Arroll B, Leveritt M, Wall C. New Zealand medical students have positive attitudes and moderate confidence in providing nutrition care: a cross-sectional survey. J Biomed Educ 2015;2015:7

6. Ball L, Desbrow B, Leveritt M. An exploration of individuals' preferences for nutrition care from Australian primary care health professionals. Aust J Primary Health 2014;20(1):113-120.

7. Bonevski B, Campbell E, Sanson-Fisher R. Primary care practitioners and health promotion: a review of current practices. Health Promot J Aust 1996;6(1):22.

8. Ball LE, Hughes RM, Leveritt MD. Nutrition in general practice: role and workforce preparation expectations of medical educators. Aust J Prim Health 2010;16(4):304-310.

9. American Dietetic Association. Nutrition and you: trends 2008. American Dietetic Association,. Chicago 2011.

10. Brotons C, Ciurana R, Piñeiro R, Kloppe P, Godycki-Cwirko M, Sammut MR. Dietary advice in clinical practice: the views of general practitioners in Europe. Am J Clin Nutri 2003;77(4):1048S-1051S. 


\section{Chapter}

\section{Nutrition in medicine: Medical students' satisfaction, perceived relevance and preparedness for practice}




\begin{abstract}
Purpose: Doctors play a critical role in providing nutrition care and supporting patients to adopt healthy dietary habits. Improving the quality of nutrition education in medical schools is necessary to build the capacity of doctors to deliver effective nutrition care to help reduce malnutrition especially for sub-Saharan Africa. This study investigated Ghanaian clinical medical students' satisfaction with their current nutrition education, preparedness to provide nutrition care, perceived relevance of nutrition education to their future practice and the associations among these variables.
\end{abstract}

Method: A survey among 207 clinical students was conducted. An 11-item questionnaire with subscales was used to assess students' demographic characteristics, satisfaction with current nutrition education, preparedness to provide nutrition care and perceived relevance of nutrition education to their future practice.

Results: Ninety-two percent $(n=187)$ of the students considered nutrition education to be relevant to their future practice. However, the majority of the students (70\%) were dissatisfied with the amount of time dedicated to nutrition education in their curriculum; integration of nutrition into organ-system based modules (62.0\%); inclusion of nutrition materials to promote independent study (62.8\%) and nutrition course content (59.0\%). Only $22.2 \%$ of the students felt adequately prepared by their current nutrition education to provide nutrition care in the general practice setting. Satisfaction with current education in nutrition was positively related to students' preparedness to provide nutrition care in the general practice setting.

Discussion: Students were dissatisfied with their current education in nutrition, felt inadequately prepared to provide nutrition care but considered nutrition education to be highly relevant to their future practice. The findings of this study provide additional evidence that suggests changes in the current format and content of nutrition education in medical education.

Key words: Nutrition education, Medical students, Satisfaction, Ghana, Sub-Saharan Africa 


\section{Introduction}

Malnutrition is a global public health problem. As affluent societies are grappling with overweight/obesity, diabetes and other chronic and non-communicable diseases; lowincome countries are confronted with rising prevalence of these chronic diseases and/or non-communicable diseases in addition to under nutrition and infectious diseases. In $2013,36.9 \%$ of men and $38.0 \%$ of women aged $\geq 20$ years were overweight globally ${ }^{1}$. Recent studies estimate the prevalence of overweight and obesity in adults to range from $10 \%$ to $40 \%$ in Ghana and Nigeria ${ }^{2-5}$. In 2011, one in seven Ghanaian children under the age of five was moderately or severely underweight; $23 \%$ moderately or severely stunted; and $6 \%$ moderately or severely wasted ${ }^{6}$. Studies report that these diseases may decline if medical doctors provide nutrition and dietary advice to their patients ${ }^{7,8}$.

Ghana is one of the countries that have signed unto the Scaling up Nutrition (SUN) movement that have outlined strategic processes to overcome malnutrition in member countries $^{9}$. Interventions to reduce micronutrient deficiencies and to tackle maternal and child undernutrition have also been outlined and widely known ${ }^{10}$. Paramount to the success of these approaches is the availability of adequately trained healthcare professionals including doctors. Evidence from the US and other high income countries consider doctors to be important and credible sources of information on health and nutrition and possess the ability to motivate their patients to adopt healthy lifestyle behaviours ${ }^{11-13}$. Doctors in the general practice setting can be effective in enhancing patients' dietary and nutrition behaviour through nutrition counselling ${ }^{14-17}$. However, the delivery of nutrition care by doctors has been reported to be less frequent ${ }^{18-21}$. Studies have indicated a disparity between doctor's beliefs in the role diet and nutrition play in the maintenance of health and the actual delivery of nutrition care ${ }^{22-25}$.

Evidently, most doctors report receiving inadequate nutrition education from medical school and as a result feel inadequately prepared and less self-efficacious to provide nutrition care ${ }^{20-28}$. Several studies also indicate that majority of medical students and incoming interns are unsatisfied with their medical nutrition education and feel unprepared to provide nutrition care to patients ${ }^{29-31}$.

Although the situation of nutrition education in medical education has been explored extensively in high income countries, it has not been frequently investigated in Ghana and other parts of sub-Saharan Africa ${ }^{32,33}$. In our search of the literature we only came across two studies investigating this phenomenon. The Sodjinou et al ${ }^{32}$ study evaluated nutrition education in medical and other health professional schools in West Africa but did not consider medical schools separately and did not also evaluate the views of medical students regarding their nutrition education. Oyewole and colleagues ${ }^{33}$ evaluated strategies through which nutrition education could be incorporated into the medical curricula in Nigeria but did not sought the views of students. Thus, studies evaluating students' perception of their nutrition education in medical education in Ghana and the rest of the sub-region are non-existent. It is also unclear to what extend the evidence reported 
from high income countries could be applied to healthcare and educational systems in Ghana and other countries of the sub region, faced with both infrastructural and human resource constraints ${ }^{34,35}$. An evaluation of this potential gap is a necessary step to designing educational interventions for nutrition education in medical education. It is also needed to build the capacity of future doctors with the needed tools to implement effective nutrition interventions to help reduce the burden of malnutrition in Ghana and in other parts of Sub-Saharan Africa. This study intends to answer the following research questions.

i. What are students' level of satisfaction with their current nutrition education and preparedness to provide nutrition care in the general practice setting?

ii. What are students' perceptions of the relevance of nutrition education to their future practice?

iii. Does students' satisfaction relate to their preparedness to provide nutrition care and perceived relevance of nutrition education?

iv. Do students' satisfaction, preparedness and relevance differ by level of training?

\section{Methods}

\section{Setting and participants}

The University for Development Studies, School of Medicine and Health Sciences (UDSSMHS) follows a problem-based learning/Community-based Education and Service (PBL/COBES) curriculum for the teaching and learning of its medical students ${ }^{36}$. Teaching and learning is organised through integrated theme-based, problem-based learning blocks. Nutrition does not have a dedicated block and is mostly taught as integrated topics during preclinical year 2 and 3 and less frequent during the clinical years. Students spend the first three years learning normal anatomy and functioning of the human body and pathophysiology of diseases in the $4^{\text {th }}$ year. Students then start a coordinated discipline-based clinical training from $5^{\text {th }}$ to $7^{\text {th }}$ year of medical school. The community-based education and service component allows students to live and work for at least 4 weeks per year in a rural community in Ghana during medical year 2 to 4 . During these periods, students work with community members, health personnel and volunteers to undertake community health diagnosis, profiling, problem identification and intervention strategies. Details of how teaching and learning activities is undertaken for the entire curriculum is published elsewhere ${ }^{36}$.

Participants of this study included only undergraduate clinical level medical students (clinical year 1-3). Our choice of clinical students was premised on the assumption that these groups of students have experienced more than $50 \%$ of the entire curriculum and will be in a better position to assess the situation of nutrition education in the curriculum. 
Ethical approval for this study was granted by the Navrongo Health Research Centre Institutional Review Board (NHRCIRB) (Ethics Approval ID: NHRCIRB209), Ghana.

\section{Recruitment and data collection procedures}

Prior to the commencement of data collection, students were informed of the study and were recruited to participate through a series of announcements that were made before or at the end of usual lecture times. Data was collected using a paper-based, self-administered questionnaire. The questionnaire was distributed to all students after an end of rotation examination. Students were required to complete and submit the questionnaire before leaving the examination room. Students were informed that their participation in the study was voluntary and they were at liberty to stop at any stage of the process. A consent form and an information sheet detailing the purpose of the study were included in the questionnaire. Students were given two pieces of candy if they returned a completed questionnaire. From a total of 215 questionnaires distributed, 207 were returned (response rate $=96 \%$ )

\section{Measures}

All data was collected using an 11-item questionnaire that covering the following:

a. Satisfaction with current education in nutrition: Students' satisfaction with the quality and quantity of their current nutrition education was assessed using six items on a 5point Likert scale in which 1 indicated very dissatisfied; $2=$ Dissatisfied; $3=$ neither satisfied nor dissatisfied; 4 = satisfied and 5 = very satisfied. Items were derived from a previously validated and widely used survey instrument ${ }^{37,38}$. This scale yielded a Cronbach's alpha of 0.79 , indicating a good level of internal consistency.

b. Perceived preparedness to provide nutrition care: Students were asked to indicate the extent to which they felt adequately prepared by their current nutrition education to provide nutrition care using a 5 -point Likert scale (i.e. $1=$ very inadequate; $2=$ inadequate; 3 = neither adequate nor inadequate; 4 =adequate and 5=very adequate).

c. Perceived relevance of nutrition education to future practice: Students were asked to what extent they perceived nutrition education to be relevant to their future practice as medical doctors using a 5-point Likert scale (1=very irrelevant; 2=irrelevant; 3=neither relevant nor irrelevant; $4=$ relevant and $5=$ very relevant).

Questions relating to format of learning about nutrition, unmet nutrition-related educational needs age, sex and level of clinical training were also included into the questionnaire. The questionnaire was reviewed by a panel of experts in nutrition and health professions education and was found to be content valid. It was also pretested on a sample of 10 students to assess understanding and comprehensibility. 


\section{Statistical analysis}

Statistical analyses were performed using IBM SPSS Statistics 21.0 and GraphPad prism version 5.0. Relationship between continuous variables and categorical variables were determined using independent t-test and one-way ANOVA where appropriate. Pearson product-moment correlation was used to examine associations between all continuous variables. A p-value of less than 0.05 was considered significant in all statistical tests of significance.

\section{Results}

\section{Demographics}

With a mean (SD) age of 25.13 (2.56) years, 59.9\% ( $n=124)$ were males, 38.2\% $(n=79)$ in clinical year 2 and $30.9 \%(n=64)$ each in both clinical year 1 and 3.

\section{Satisfaction with the quality and quantity of current education in nutrition}

The majority of students were dissatisfied with all aspects of their nutrition education assessed (Shown in Table 1). Clinical year three (10.25 (4.08)) students were more satisfied $(F(1,196)=5.01, p=0.01, \eta 2=0.05))$ with the quality and quantity of their current nutrition education than clinical year one $(8.70(3.20))$ and two $(8.21$ (3.92)) students.

Table 1: Students' perceived satisfaction with the quality and quantity of their current education in nutrition

\begin{tabular}{|c|c|c|c|c|}
\hline Perceived satisfaction (Max. score $=20$ ) & Mean (SD) & Dissatisfied & $\begin{array}{l}\text { Neither satisfied } \\
\text { or dissatisfied }\end{array}$ & Satisfied \\
\hline $\begin{array}{l}\text { Amount of time dedicated for nutrition } \\
(n=202)\end{array}$ & $2.15(1.06)$ & $141(69.8 \%)$ & $32(15.8 \%)$ & $29(14.4 \%)$ \\
\hline $\begin{array}{l}\text { Integration of nutrition content into organ- } \\
\text { system based blocks }(n=200)\end{array}$ & $2.32(1.11)$ & $124(62.0 \%)$ & $39(19.5 \%)$ & $37(29.8 \%)$ \\
\hline $\begin{array}{l}\text { Inclusion of materials to promote } \\
\text { independent study of nutrition }(n=199)\end{array}$ & $2.20(1.09)$ & $125(62.8 \%)$ & $47(23.6 \%)$ & $27(13.6 \%)$ \\
\hline Nutrition course content $(n=200)$ & $2.32(1.09)$ & $118(59.0 \%)$ & $50(25.0 \%)$ & $32(16.0 \%)$ \\
\hline $\begin{array}{l}\text { Mean (SD) perceived satisfaction score } \\
(n=197)\end{array}$ & $8.95(3.83)$ & & & \\
\hline
\end{tabular}

Frequencies do not add up to 207 due to missing responses.

\section{Students' perceived preparedness to provide nutrition care}

Reporting a mean (SD) preparedness score of 2.55 (1.08), only $22.2 \%(n=45)$ of the students said they felt adequately prepared to provide nutrition care in the general practice setting, $51.7 \%(n=105)$ felt inadequately prepared, and $26.1 \%(n=53)$ were unsure. These 
results did not differ by level of clinical training $(F(2,200)=2.43, p=0.09, \eta 2=0.02))$. A large majority (86.4\%) of the students said they will benefit from further training in nutrition education. Clinical year one (90.6\%) and two (91.0\%) students were more likely $(\eta 2$ $=0.18, p=0.04)$ than clinical year three $(75.4 \%)$ students to say they will benefit from further training in nutrition.

\section{Perceived relevance of nutrition education to future practice}

Students recorded a mean (SD) score of 4.18 (0.97) (maximum score $=5$ ) with less than 10 percent saying nutrition education was irrelevant or were unsure. Students' responses did not differ by level of clinical training $(F(2,201)=1.60, \eta 2=0.02, p=0.20)$.

\section{Format of learning nutrition and preferred format of nutrition education}

As shown in Table 2, majority (86.4\%) of the students said they will benefit from further training in nutrition education if the opportunity with $60 \%$ saying they will prefer such training from a nutritionists/dietician. Clinical year one (90.6\%) and two (91.0\%) students were more likely $(\eta 2=0.18, p=0.04)$ than clinical year three $(75.4 \%)$ students to say they will benefit from further training in nutrition.

Table 2: Format of nutrition education and students' preferred format of nutrition education

\begin{tabular}{ll}
\hline Variable & Frequency (\%) \\
\hline Current format of learning about nutrition & \\
Separate course in nutrition $(\mathrm{n}=205)$ & $26(12.7 \%)$ \\
Lectures on selected topics in nutrition ( $\mathrm{n}=203)$ & $147(72.4 \%)$ \\
Nutrition concepts integrated into course work/block ( $\mathrm{n}=203)$ & $120(59.1 \%)$ \\
Nutrition-related educational needs & \\
Has unmet nutrition-related educational need & $143(71.9 \%)$ \\
Will benefit from further training in nutrition & $172(86.4 \%)$ \\
Students' preferred format of learning about nutrition ( $\mathrm{n}=196)$ & $122(62.2 \%)$ \\
Training provided by a nutritionists/dietician in the general practice setting & $64(32.7 \%)$ \\
Dedicated courses for nutrition & $10(5.1 \%)$ \\
Online training programmes
\end{tabular}

Relationship between satisfaction, perceived preparedness and relevance of nutrition education

Using Pearson correlation analysis satisfaction correlated with preparedness to provide nutrition care $(r=0.489, p<0.001)$ (Shown in table 3$)$. However, there was no significant correlation between perceived preparedness and relevance $(r=0.046, p=0.356)$ as well as satisfaction and relevance $(r=-0.032, p=0.485)$. 


\section{Discussion}

\section{General discussion}

In this study we assessed clinical medical students' satisfaction with their current nutrition education, perceived preparedness to provide nutrition care and their perceptions of the relevance of nutrition education to their future practice.

In agreement with previous studies majority of the students considered their nutrition education to be inadequate. ${ }^{24,29,30,38-41}$ Given the current situation one may recommend increasing the instruction time and content of nutrition education in the curriculum, however this may be problematic due to complaints of the medical curriculum being overloaded ${ }^{32}$ and matters of priorities. Adoption of a multifaceted curriculum for nutrition education that brings to bear the basic principles of nutrition and their application to clinical practice and the development of a dedicated nutrition course supported by a comprehensive integration of nutrition content throughout the curriculum may be a better option ${ }^{42}$.

Similar to findings from other parts of the world, a large proportion of the students felt unprepared by their current nutrition education to provide nutrition care ${ }^{43-45}$. This is a concern because we may be producing doctors who feel inadequate to provide nutrition counselling to their patients and to make appropriate clinical decisions on nutritionrelated issues ${ }^{28,46,47}$.

Unsurprisingly, and in consonance with previous studies $30,31,38,41,48$, most of the students regarded nutrition education to be highly relevant to their future practice. This demonstrates the high value students place on nutrition education and may utilize every opportunity given them to learn about nutrition. Curriculum planners and medical educators appear not to make use of this opportunity to improve nutrition education as the status of nutrition education in medical education is still questionable. ${ }^{32,49,50}$

Giving credence to inter-professional collaboration in nutrition education, most of the students said they preferred training provided by a dietician/nutritionist in the hospital setting to help meet their unmet nutrition-related educational needs. Inter-professional collaboration to provide nutrition education to medical students is very critical towards improving the delivery of nutrition care. ${ }^{25,51,52}$ This is however confronted with barriers such as the lack of faculty trained in nutrition, lack of physician nutrition specialists or other nutrition educators on faculty as these professionals serve as role models to both medical students and residents for addressing nutrition in patient interactions. ${ }^{53-55}$

Importantly, we found that students who were more satisfied with their current nutrition education felt more adequately prepared to provide nutrition care in the general practice setting. This is similar to the findings reported by Mihalynuk et al ${ }^{38}$ who found positive correlations between perceived quality of nutrition education and self-reported nutrition proficiency in a sample of practicing family physicians in Washington State. 
Thus, improving students' satisfaction in nutrition education may be important towards improving preparedness and confidence to provide nutrition care.

Although, students were generally unsatisfied with their current nutrition education, their satisfaction differed by level of clinical training. Clinical year three students compared to clinical year one and two students reported being more satisfied with their current nutrition education. Notwithstanding the absence of a linear trend, students in the junior years of clinical training might have been less satisfied with their current nutrition education so far because they were yet to be exposed to some aspects of the curriculum that those in clinical year three have already experienced.

Contrary to the findings of Spencer et al ${ }^{30}$ students perception of the relevance of nutrition education to their future practice did not differ by level of clinical training. The lack of differences in this study could be due to the inclusion of only clinical level students who may be sharing similar perceptions or to the more urgent and visible need for nutrition care in African countries than in high income countries.

\section{Implications to practice and future studies}

Our findings add to the evidence that nutrition is inadequate in the medical curriculum. It provides important insights into avenues that could inform future curriculum planning and development. Improving students' satisfaction and adequacy of nutrition education are some of the avenues curriculum planners could utilise. Given that this is the first study in Ghana and in the sub-region to evaluate the nutrition education of medical students; its findings serve as a basis for future studies in this subject. They may stimulate discussions and research regarding this topic among medical educators in Ghana and sub-Saharan Africa. Future research should explore the influence of the current findings on students' nutrition-related knowledge, attitudes towards, and self-efficacy in nutrition care. In addition, studies should explore qualitatively students' opinions on the factors that may be contributing to the inadequacy of nutrition education. Meanwhile, innovative teaching and learning methodologies should be adopted for nutrition education. Interprofessional collaboration in the teaching and learning of nutrition should also be encouraged.

\section{Strengths and Limitations}

The use of previously validated survey items and nutrition experts to examine the content validity of the survey items enhanced confidence in the findings of the study. Furthermore, using an instrument that is based on items relevant to nutrition issues of the study setting may help facilitate the recognition and prioritization of nutrition content in medical education.

Our study is not without limitations. Its cross-sectional nature makes it difficult to establish causality. Nonetheless it gives a snapshot of the situation of nutrition education in Ghana and in the sub-region. This study reports on the nutrition education of a single 
medical school. This makes it difficult to generalize its findings. As an obvious limitation of survey-based studies, the findings of this study may be subject to social desirability bias. However, the questionnaires were self-administered and most students gave selfcritical responses to the survey items, thereby, minimising the effect of this bias on the findings.

\section{Conclusion}

Students regarded nutrition to be relevant to their future practice, felt unsatisfied with the quality and quantity of their current nutrition education and inadequately prepared to provide nutrition care. Satisfaction with the quality and quantity of nutrition education may be important in making students feel adequately prepared to provide nutrition care. Level of clinical training may also be important in determining students' satisfaction with their nutrition education.

\section{Disclosure}

None

\section{Ethical Approval}

Ethical approval has been granted by the Navrongo Health Research Centre Institutional Review Board (NHRCIRB) (Ethics Approval ID: NHRCIRB209), Ghana.

\section{Other disclosure}

None

\section{Acknowledgement}

Authors wish to thank the students of the University for Development Studies, School of Medicine and Health Sciences for their support and acceptance to take part in the research. 


\section{References}

1. Ng M, Fleming $\mathrm{T}$, Robinson $\mathrm{M}$, et al. Global, regional, and national prevalence of overweight and obesity in children and adults during 1980-2013: a systematic analysis for the Global Burden of Disease Study 2013. Lancet 2014; 384(9945):766-81

2. Mogre V, Mwinlenaa P, Oladele J, Amalba A. Impact of physical activity levels and diet on central obesity among civil servants in Tamale metropolis. J. Med Biomed Sci 2012;1(2):1-9

3. Mogre V, Atibilla J, Kandoh B. Association between breakfast skipping and adiposity status among civil servants in the Tamale metropolis. J Med Biomed Sci.2013;2(3):1-7.

4. Benkeser R, Biritwum R, Hill A. Prevalence of overweight and obesity and perception of healthy and desirable body size in urban, Ghanaian women. Ghana Med J 2012;46(2):66-75.

5. Chukwuonye II, Chuku A, John C, et al. Prevalence of overweight and obesity in adult Nigerians-a systematic review. Diabetes Metab Syndr Obes 2013;6: 43-47.

6. Ghana Statistical Service.Ghana Multiple Indicator Cluster Survey with an Enhanced Malaria Module, 2011, Final Report and Biomarker.Ghana Statistical Service, Accra, Ghana. 2011

7. Horrocks P, Blackmore R, Wright A. A Long-term Follow-up of Dietary Advice in Maturity Onset Diabetes: The Experience of One Centre in the UK Prospective Study. Diabet Med 1987;4(3):241-244.

8. Ammerman AS, Devellis RF, Carey TS, et al. Physician-based diet counseling for cholesterol reduction: current practices, determinants, and strategies for improvement. Prev. Med 1993;22(1):96-109.

9. Sun Movement. The SUN Movement Strategy and Roadmap (2016-2020) Available at : (http://scalingupnutrition.org/sun-countries/ghana/); Accessed 23092016

10. Bhutta ZA, Das JK, Rizvi A, et al. Evidence-based interventions for improvement of maternal and child nutrition: what can be done and at what cost? Lancet 2013 ;382(9890):452-477.

11. American Dietetic Association. Nutrition and you: Trends 2008. Report of Results. Chicago, American Dietetic Association. 2008.

12. American Dietetic Association. Nutrition and you: Trends 2011. Report of Results.Chicago, American Dietetic Association. 2011.

13. Abramson S, Stein J, Schaufele M, Frates E, Rogan S. Personal exercise habits and counseling practices of primary care physicians: a national survey. Clin J Sport Med 2000;10(1):40-48.

14. Keyserling TC, Ammerman AS, Davis C, Mok MC, Garrett J, Simpson Jr R. A randomized controlled trial of a physician-directed treatment programme for low-income patients with high blood cholesterol: the Southeast Cholesterol Project. Arch Fam Med 1997;6(2):135.

15. Ockene JK, Ockene IS, Quirk ME, et al. Physician training for patient-centered nutrition counseling in a lipid intervention trial. Prev. Med 1995;24(6):563-570.

16. Ockene IS, Hebert JR, Ockene JK, et al. Effect of physician-delivered nutrition counseling training and an office-support programme on saturated fat intake, weight, and serum lipid measurements in a hyperlipidemic population: Worcester Area Trial for Counseling in Hyperlipidemia (WATCH). Arch Intern Med 1999;159(7):725-731.

17. Ball L, Johnson C, Desbrow B, Leveritt M. General Practitioners can offer effective nutrition care to patients with lifestyle related chronic disease. J Prim Health Care 2013;5(1):59-69.

18. Mowe M, Bosaeus I, Rasmussen HH, Kondrup J, Unosson M, Irtun $\varnothing$. Nutritional routines and attitudes among doctors and nurses in Scandinavia: a questionnaire based survey. Clin Nutr 2006;25(3):524-532.

19. Collier R. Canadian medical students want more nutrition instruction. CMAJ 2009;181(3-4):133-134.

20. Temple NJ. Survey of nutrition knowledge of Canadian physicians. J Am Coll Nutr 1999;18(1):26-29.

21. Weinsier RL, Boker JR, Morgan SL, et al. Cross-sectional study of nutrition knowledge and attitudes of medical students at three points in their medical training at 11 southeastern medical schools. Am J Clin Nutr 1988;48(1):1-6.

22. Kolasa K. Developments and challenges in family practice nutrition education for residents and practicing physicians: an overview of the North American experience. Eur J Clin Nutr 1999;53:S89-S96. 
23. Leslie FC, Thomas S. Symposium 9: Competent to care Are all doctors competent in nutrition? Proc Nutr Soc 2009;68(3):296.

24. Adams KM, Kohlmeier M, Zeisel SH. Nutrition Education in US Medical Schools: Latest Update of a National Survey. Acad Med 2010;85(9):1537-1542.

25. Glanz K. Review of nutritional attitudes and counseling practices of primary care physicians. Am J Clin Nutr 1997;65(6):2016S-2019S.

26. Cimino JA. Why can't we educate doctors to practice preventive medicine? Prev. Med 1996;25(1):63-65.

27. Soltesz KS, Price JH, Johnson LW, Tellijohann SK. Family physicians' views of the preventive services task force recommendations regarding nutritional counseling. Arch Fam Med 1995;4(7):589.

28. Kushner RF. Barriers to providing nutrition counseling by physicians: a survey of primary care practitioners. Prev Med 1995;24(6):546-552.

29. Weinsier RL, Boker JR, Feldman EB, Read MS, Brooks CM. Nutrition knowledge of senior medical students: a collaborative study of southeastern medical schools. Am J Clin Nutr 1986;43(6):959-968.

30. Spencer EH, Frank E, Elon LK, Hertzberg VS, Serdula MK, Galuska DA. Predictors of nutrition counseling behaviors and attitudes in US medical students. Am J Clin Nutr 2006;84(3):655-662.

31. Vetter ML, Herring SJ, Sood M, Shah NR, Kalet AL. What do resident physicians know about nutrition? An evaluation of attitudes, self-perceived proficiency and knowledge. J Am Coll Nutr 2008;27(2):287-298.

32. Sodjinou R, Bosu WK, Fanou N, et al. Nutrition training in medical and other health professional schools in West Africa: the need to improve current approaches and enhance training effectiveness. Glob Health Action 2014; 7:24827

33. Oyewole O, Atinmo T. Nutrition education in medical training: the need to reconsider the sacrosanctity of medical education in Nigeria. Afr J Med Med Sci 2008;37(3):219-224.

34. Dare L, Buch E. The future of health care in Africa. BMJ 2005;331(7507):1-2.

35. Willis-Shattuck M, Bidwell P, Thomas S, Wyness L, Blaauw D, Ditlopo P. Motivation and retention of health workers in developing countries: a systematic review. BMC Health Serv Res 2008;8(1):247.

36. Mogre V, Amalba A, Saaka M, Kyei-Aboagye K. Medical students' achievement on the Bachelor of Medicine, Bachelor of Surgery/Chirurgery (MBChB) part I and II licensing examination: a comparison of students in problem-based learning community-based education and service and conventional curricula in Ghan. J Educ Eval Health 2014;11:10.

37. Walsh CO, Ziniel SI, Delichatsios HK, Ludwig DS. Nutrition attitudes and knowledge in medical students after completion of an integrated nutrition curriculum compared to a dedicated nutrition curriculum: a quasi-experimental study. BMC Med Educ 2011;11:58.

38. Mihalynuk TV, Coombs JB, Rosenfeld ME, Scott CS, Knopp RH. Survey correlations: Proficiency and adequacy of nutrition training of medical students. J Am Coll Nutr 2008;27(1):59-64.

39. Walker WA. Advances in nutrition education for medical students - Overview. Am J Clin Nutr 2000;72(3):865S-867S.

40. Gramlich LM, Olstad DL, Nasser R, et al. Medical students' perceptions of nutrition education in Canadian universities. Appl Physiol Nutr Metab 2010;35(3):336-343.

41. Hyska J, Mersini E, Mone I, et al. Assessment of knowledge, attitudes and practices about public health nutrition among students of the University of Medicine in Tirana, Albania. South East Euro J Public Health 2014;1

42. DiMaria-Ghalili RA, Edwards M, Friedman G, et al. Capacity building in nutrition science: revisiting the curricula for medical professionals. Ann N Y Acad Sci 2013;1306(1):21-40.

43. Hardman WE, Miller BL, Shah DT, Nowson C. Student Perceptions of Nutrition Education at Marshall University Joan C. Edwards School of Medicine: A Resource Challenged Institution. J Biomed Educ 2015;2015:8

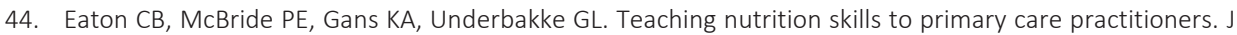
Nutr 2003;133(2):563S-566S.

45. Jay M, Gillespie C, Ark T, et al. Do internists, pediatricians, and psychiatrists feel competent in obesity care? J Gen Intern Med 2008;23(7):1066-1070. 
46. Mihalynuk TV, Scott CS, Coombs JB. Self-reported nutrition proficiency is positively correlated with the perceived quality of nutrition training of family physicians in Washington State. Am J Clin Nutr 2003;77(5):1330-1336.

47. Mihalynuk TV, Knopp RH, Scott CS, Coombs JB. Physician informational needs in providing nutritional guidance to patients. Fam Med 2004;36(10):722-726.

48. Crowley J, Ball L, Han DY, Arroll B, Leveritt M, Wall C. New Zealand Medical Students Have Positive Attitudes and Moderate Confidence in Providing Nutrition Care: A Cross-Sectional Survey. J Biomed Educ 2014.

49. Adams KM, Kohlmeier M, Powell M, Zeisel SH. Nutrition in Medicine: Nutrition Education for Medical Students and Residents. Nutr Clin Pract 2010;25(5):471-480.

50. Devries S, Dalen JE, Eisenberg DM, et al. A Deficiency of Nutrition Education in Medical Training. Am J Med 2014;127(9):804-806.

51. Eisenberg DM, Burgess JD. Nutrition Education in an Era of Global Obesity and Diabetes: Thinking Outside the Box. Aca Med 2015; 90(7):854-60.

52. Eisenberg DM, Miller AM, McManus K, Burgess J, Bernstein AM. Enhancing medical education to address obesity: "See one. Taste one. Cook one. Teach one.". JAMA Intern Med 2013;173(6):470-472.

53. Touger-Decker R. Nutrition education of medical and dental students: innovation through curriculum integration. Am J Clin Nutr 2004;79(2):198-203.

54. Wong V, Millen BE, Geller AC, Rogers AE, Maury J, Prout MN. What's in store for medical students? Awareness and utilization of expert nutrition guidelines among medical school preceptors. Prev. Med 2004;39(4):753-759.

55. Intersociety Professional Nutrition Education Consortium. Bringing physician nutrition specialists into the mainstream: rationale for the Intersociety Professional Nutrition Education Consortium. Am J Clin Nutr 1998;68(4):894-898. 



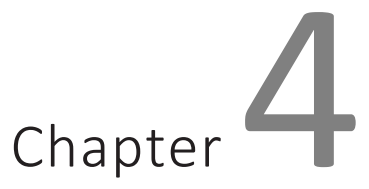

Future doctors' nutrition-related knowledge, attitudes and self-efficacy regarding nutrition care in the general practice setting: A crosssectional survey 


\section{Abstract}

Background: Doctors are in a good position to provide nutrition advice to patients. However, doctors and medical students find their nutrition education to be inadequate. We evaluated nutrition-related knowledge, attitudes and self-efficacy in a sample of future doctors. Furthermore, we investigated the association between nutrition-related knowledge, attitude and self-efficacy. We also compared nutrition-related knowledge, attitudes and self-efficacy with level of clinical training.

Methods: Following a cross-sectional design, the nutrition-related knowledge, attitudes and self-efficacy of 207 undergraduate clinical level medical students (referred to as future doctors) was measured using a questionnaire. Items of the questionnaire were derived from previously validated survey instruments. Descriptive statistics of mean and standard deviation was used to describe the data.

Results: Future doctors had a mean knowledge score of $64 \%$. Their mean knowledge scores in the nutrition topics assessed were 41\% for 'malnutrition in children', 59\% for 'diabetes and obesity' and 73\% for 'nutrients, energy and their deficiencies'. Future doctors' attitudes towards nutrition care were generally positive but were they uncertain in the effectiveness of health education in changing the lifestyle of patients. They felt inadequate in their self-efficacy to provide nutrition care. Attitudes towards nutrition-care correlated $(r=0.371, p<0.001)$ positively with self-efficacy to provide nutrition care. Level of clinical training was associated to nutrition-related knowledge of the students.

Conclusion: Future doctors had positive attitudes towards nutrition care but showed important knowledge gaps and also felt inadequate in their confidence to provide nutrition care. Attitudes may be important in nutrition care self-efficacy.

Key words: Attitudes, Confidence, Medical education, Nutrition education, Ghana and Self-efficacy. 


\section{Introduction}

There is growing concern for the rising trend of nutrition and diet related diseases in many sub-Saharan African countries. Under nutrition, diabetes, overweight and obesity are diet-related are now diseases of public health concern in the sub-region ${ }^{1,2}$. Many children (28 million) in Central and West Africa are stunted, underweight or wasted ${ }^{3,4}$.On the other hand, diabetes, overweight and obesity have also been estimated to be rising in the sub-region with close to 14.2 million people affected and estimated to rise to 34 million by $2040^{1}$.

Under nutrition in the first 1000 days of a child's life is associated with mortality, morbidity, impaired cognitive ability and poor school performance ${ }^{4-6}$ and is strongly associated with the development of non-communicable diseases such as overweight and obesity, diabetes and hypertension.

In a sub-region where rising prevalence of diet-related diseases could increase the health burden in terms of morbidity and costs related to the treatment or care of patients, improving nutrition could lead to a reduction in the situation. Nutrition educators, specialists, and multidisciplinary support teams are necessary to cater for the needs of patients in sub-Saharan Africa in order to reduce the ever increasing epidemics. Nevertheless, among all the identifiable human resources, medical doctors are regularly considered as credible sources of nutrition advice ${ }^{7-10}$ and are better placed to provide dietary and nutrition advice to patients.

In evaluating the status of nutrition education in the medical curricula, a number of studies in the US ${ }^{11-13}$ and other high income countries ${ }^{10,14,15}$ have found it to be inadequate. This has been corroborated by medical doctors reporting inadequate training in nutrition education during medical school, inadequate knowledge and skills in nutrition and feeling less efficacious in their ability to effectively provide nutrition care to patients 10,16-19. A number of factors have been noted for this situation including time limitations in the medical curriculum, resistance to the addition of new courses or lectures, and low priority for nutrition education ${ }^{20-23}$. Other factors include the curriculum emphasizing on disease treatment but not disease prevention, and lack of physician nutrition specialists or other nutrition educators on faculty as these professionals serve as role models to both medical students and residents for addressing nutrition in patient interactions ${ }^{24-26}$.

Unlike in the USA and other developed countries, the issue of nutrition in medical education is scarcely explored in countries of sub-Saharan Africa, where increased prevalence of diet and nutrition-related diseases are persistent. In such settings, it would be most imperative to conduct an evaluation of the situation of nutrition education, the confidence, knowledge and attitudes of future medical doctors as a necessary step towards empowering them to be able to provide nutrition care to prevent and reduce the burden of diet-related diseases ${ }^{27-33}$. The findings of a recent realist review of educational interventions to improve nutrition care competencies and delivery by doctors and other 
healthcare professionals highlighted the importance of undertaking a needs assessment in this direction ${ }^{34}$.

Previous findings have been equivocal in relating attitudes with knowledge and selfefficacy in nutrition ${ }^{18,35-37}$. Although one study has evaluated the association between attitudes, self-perceived proficiency and knowledge among a sample of internal medicine residents in the US ${ }^{35}$ no study have evaluated the relationship between knowledge, attitudes and self-efficacy in nutrition among undergraduate medical students. Thus studies evaluating how these are related among future doctors are lacking.

Undertaking a needs assessment of future doctors' nutrition-related knowledge, attitudes and self-efficacy in sub-Saharan Africa using Ghana as a case example is likely to increase our understanding of the situation of nutrition education in sub-Saharan Africa. It will also provide baseline data to teachers, nutrition practitioners, researchers in health professions education and policy makers to inform efforts towards reforms in the medical curricula in the sub-region.

We thus intend to answer the following research questions.

1. What are future doctors' nutrition-related knowledge, attitudes towards, and self-efficacy in nutrition care?

2. Does future doctors' nutrition-related knowledge relate to their attitudes towards, and self-efficacy in nutrition care?

3. Does level of clinical training relate to future doctors' nutrition-related knowledge, attitudes towards, and self-efficacy in nutrition care?

\section{Methods}

\section{Ethics Statement}

All data collection methods and procedures complied with the guidelines of the Navrongo Health Research Centre Institutional Review Board (NHRCIRB) (Ethics Approval ID: NHRCIRB209) in Ghana, which subsequently approved the study. Each participant signed an informed consent form included in the questionnaire. All informed consent procedures were approved by the NHRCIRB.

\section{Study design and setting}

Following a cross-sectional design, participants of the study included undergraduate clinical level medical students (referred to as future doctors in this study) of the University for Development Studies, School of Medicine and Health Sciences (UDS-SMHS), Ghana. Teaching and learning is organised around a problem-based learning, community-based education and service (PBL/COBES) curriculum. The PBL/COBES curriculum is implemented through a series of theme-based PBL blocks for the preclinical years and coordi- 
nated discipline-based clerkship rotations for the clinical years of training. Topics in nutrition are usually covered in the preclinical years and sparsely taught in the clinical years. Further information on the structure of medical education of the UDS-SMHS is published elsewhere ${ }^{38,39}$.

\section{Recruitment and data collection procedures}

All future doctors were eligible to participate in the study. Prior to the commencement of data collection, students were informed about the study through announcements that were made before or at the end of usual lecture times depending on the time convenient for the announcement to be made. All data was collected using a paper-based anonymous survey instrument. Future doctors completed the survey after an end of rotation examination and returned it. The survey included information about the study and an informed consent to be signed before consenting to participate in the study. Voluntary participation was encouraged and students were assured of the confidentiality of their responses. Future doctors were incentivised with two pieces of candy upon the return of a completed survey instrument.

\section{Measures}

The survey instrument was used to assess future doctors' nutrition-related knowledge, attitudes towards, and self-efficacy in nutrition care (shown in table 1). Nutrition-related knowledge was assessed using 10 multiple choice questions. Topics covered were 'nutrients, energy and their deficiencies' ( 6 items); 'malnutrition in children' ( 2 items) and 'diabetes and obesity' ( 2 items). Items were adapted from previously validated nutrition knowledge tests ${ }^{35,40}$ and informed by common nutrition issues in Ghana. Each respondent was assigned a score of 1 for every correct answer, summed and computed out of $100 \%$. Future doctors' attitudes and self-efficacy regarding nutrition were evaluated using 11-item and 9-item measures respectively. Items for the attitude measure were derived from a previously validated Nutrition in Patient Care Survey (NIPS) ${ }^{41}$. This measure had a Cronbach's alpha of 0.76. Possible responses spanned from strongly disagree to strongly agree on a 5-point Likert Scale. Items for the self-efficacy measure were also adapted from previously published surveys ${ }^{35,42,43}$. This scale had a Cronbach's alpha of 0.84 . Possible responses ranged from being very unconfident to being very confident on a 5-point Likert scale. From a list of three nutrition education resources (undergraduate medical curriculum, seminars/conferences and reading and self-directed learning), students were asked to determine the extent to which they felt those training resources contributed to their current nutrition-related knowledge. This item was derived from a previous survey ${ }^{44}$. Possible responses ranged from none to maximum on a 5-point Likert scale. Demographic characteristics such as gender and age were also assessed using the survey instrument. The final list of items was reviewed by a panel of experts and researchers in nutrition and health professions education and was found to be content valid. It 
was also pretested on a sample of 10 future doctors to assess understanding and comprehensibility. The responses of these participants were excluded from the final analysis.

Table 1: Description of survey sections

\begin{tabular}{ll}
\hline Section & Description of questions \\
\hline Knowledge & 10 questions evaluating future doctors' knowledge in nutrition \\
Attitudes & 11 questions exploring future doctors' attitudes towards nutrition \\
Self-efficacy & 9 questions evaluating self-perceived confidence in providing nutrition \\
& care \\
Nutrition education resources & 1 question exploring nutrition education resources commonly used by \\
& the future doctors \\
Demographics & 3 questions assessing students age, sex and level of clinical training \\
\hline
\end{tabular}

\section{Statistical analysis}

Statistical analyses were performed using IBM SPSS Statistics 21.0. Descriptive statistics of mean and standard deviation were used to explore and describe the data. Relationship between continuous variables and categorical variables were determined using independent $t$ test and one-way ANOVA where appropriate. Pearson product-moment correlation was used to examine associations among continuous variables. A $p$ value of less than 0.05 was considered significant in all statistical tests of significance. Figure was drawn using GraphPad prism.

\section{Results}

\section{Demographics}

From a total of 215 questionnaires distributed, 207 were returned (response rate $=96 \%$ ). With a mean age of $25.13 \pm 2.56$ years, $59.9 \%(n=124)$ were males, 38.2\% ( $n=79)$ in clinical year two and $30.9 \%(n=64)$ each in clinical year one and three.

\section{Nutrition-related knowledge}

Future doctors had an average knowledge score of 64\% (shown in Table 2). Except for the 'malnutrition in children' topic, future doctors' mean scores in all of the individual topics were above average. Future doctors' scores in all the three nutrition topics differed by level of clinical training. In the 'nutrients, energy and their deficiencies' topic, future doctors in clinical year one $(77.6 \pm 13.0)$ scored significantly $(p=0.017)$ higher (vs and,) than those in clinical year two $(73.3 \pm 13.0)$ and three $(69.6 \pm 20.0)$. Clinical year 3 students had higher mean scores than those in clinical year 1 (74.6 \pm 35.5 vs $28.0 \pm 33.2$ and 28.2 $\pm 32.8, p<0.001$ ) and 2 in the 'malnutrition in children' topic. In addition, clinical year 3 
students had higher mean scores than clinical year 1 ( $69.3 \pm 31.0$ vs $55.5 \pm 30.0$ and 55.1 $\pm 30.8, p=0.015$ ) and 2 students in the 'diabetes and obesity' topic.

Table 2: Future doctors mean scores based on nutrition-related knowledge domains ( $n=199)$

\begin{tabular}{ll}
\hline Nutrition topic & Mean \pm SD) $(n=199)$ \\
\hline Nutrients, energy and their deficiencies (6 items) & $73 \pm 15.5$ \\
Malnutrition in children (2 items) & $41 \pm 39.5$ \\
Diabetes and obesity (2 items) & $59 \pm 31.0$ \\
All topics (10 items) & $64.3 \pm 16.3$ \\
\hline
\end{tabular}

Responses do not add up to 207 due to non-response for some of the items.

\section{Attitudes towards nutrition care}

Future doctors had a mean \pm SD attitude score of $41.13 \pm 5.39$ (maximum score $=55$ ). Almost $50 \%$ of the future doctors either disagreed or were uncertain of the effectiveness of health education in promoting patients' adherence to healthy lifestyle recommendations (Shown in Table 3). Also, a substantial proportion of the future doctors were uncertain or disagreed in their competence to provide nutrition advice to patients. Future doctors' attitudes did not differ by level of clinical training $(p=0.243)$. 
Table 3: Future doctors' attitudes towards nutrition care

\begin{tabular}{|c|c|c|c|c|}
\hline Nutrition care attitude items & Mean \pm SD & Disagree & $\begin{array}{l}\text { Neither agree } \\
\text { nor disagree }\end{array}$ & \\
\hline $\begin{array}{l}\text { Discussing physical activity and nutrition information } \\
\text { with patients in the general practice is my } \\
\text { responsibility } \\
(n=200)^{\text {a }}\end{array}$ & $3.71 \pm 0.95$ & $27(13.5 \%)$ & $26(13.0 \%)$ & $147(73.5 \%)$ \\
\hline $\begin{array}{l}\text { Nutrition assessment should be included in any } \\
\text { routine appointment, just like diagnosis and treatment } \\
(\mathrm{n}=198)\end{array}$ & $3.73 \pm 0.87$ & $20(10.1 \%)$ & $36(18.2 \%)$ & $142(71.7 \%)$ \\
\hline $\begin{array}{l}\text { Nutrition counselling should be part of routine care } \\
\text { for all physicians }(n=198)\end{array}$ & $3.76 \pm 0.91$ & $20(10.1 \%)$ & $36(18.2 \%)$ & $142(71.7 \%)$ \\
\hline $\begin{array}{l}\text { Nutrition counselling is not effective use of my } \\
\text { professional time }(n=195)^{b}\end{array}$ & $3.64 \pm 1.08$ & $130(66.7 \%)$ & $29(14.9 \%)$ & $36(18.5 \%)$ \\
\hline $\begin{array}{l}\text { Severe acute malnutrition represents a medical } \\
\text { emergency }(n=198)\end{array}$ & $4.22 \pm 0.87$ & $9(4.5 \%)$ & $24(12.1 \%)$ & 165(83.3\%) \\
\hline $\begin{array}{l}\text { Patient motivation is essential to achieving dietary } \\
\text { change }(n=199)\end{array}$ & $3.98 \pm 0.87$ & $14(7.0 \%)$ & $18(9.0 \%)$ & $167(83.9 \%)$ \\
\hline $\begin{array}{l}\text { Patient will adopt a healthier lifestyle if counselled to } \\
\text { do so }(n=198)\end{array}$ & $3.83 \pm 0.84$ & $12(6.1 \%)$ & $35(17.7 \%)$ & $151(76.3 \%)$ \\
\hline $\begin{array}{l}\text { Physicians can have an effect on patients' dietary } \\
\text { behaviour if they take the time to discuss the problem } \\
(n=200)\end{array}$ & $3.98 \pm 0.72$ & $7(3.5 \%)$ & $17(8.5 \%)$ & $176(88.0 \%)$ \\
\hline $\begin{array}{l}\text { Most patients will try to change their lifestyle if I } \\
\text { advise them to do so }(n=200)\end{array}$ & $3.87 \pm 0.75$ & $10(5.0 \%)$ & $32(16.0 \%)$ & $158(79.0 \%)$ \\
\hline $\begin{array}{l}\text { I believe I have adequate competence to provide } \\
\text { nutrition advice to patients }(n=199)\end{array}$ & $3.05 \pm 0.97$ & $56(28.1 \%)$ & $74(37.2 \%)$ & $69(34.7 \%)$ \\
\hline $\begin{array}{l}\text { For most patients, health education does little to } \\
\text { promote adherence to a healthy lifestyle }(n=198)^{\text {a }}\end{array}$ & $3.35 \pm 1.05$ & $100(50.5 \%)$ & $51(25.8 \%)$ & $47(23.7 \%)$ \\
\hline Total attitude score $(n=190)$ & $41.13 \pm 5.39$ & & & \\
\hline
\end{tabular}

In computing frequencies, strongly disagree and disagree were combined to yield 'Disagree' and strongly agree and agree were combined to yield 'Agree'. Responses do not add up to 207 due to non-response to some of the items

${ }^{a}$ Response scale: 1 = strongly disagree; 2 =disagree; 3 = Neither agree nor disagree; 4 = Agree; and $5=$ Strongly agree. ${ }^{b}$ Reverse scored $=5=1 ; 4=2 ; 4=2 ; 1=5$

\section{Self-efficacy in nutrition care}

Future doctors had a mean \pm SD self-efficacy score of $29.88 \pm 5.67$ (maximum score $=45$ ). As shown in Table 4, the majority of the future doctors were either less confident or uncertain in their ability to: recommend dietary patterns for patients with type 2 diabetes (54.6\%, $n=107)$; manage severely malnourished children according to the WHO guidelines $(61.9 \%, n=122)$; provide examples of serving sizes, discussing essential fatty acids and heart health $(71.9 \%, \mathrm{n}=141)$; discuss calories per gram of protein, carbohydrates and fat and their basic metabolic roles with patients $(65.0 \%, n=128)$ and provide nutrition education for a patient recently diagnosed with HIV infection $(62.4 \%, n=123)$. Nutrition care self-efficacy scores did not differ by level of clinical training $(p=0.086)$. 
Table 4: Future doctors' self-efficacy in providing nutrition care in the general practice setting

\begin{tabular}{|c|c|c|c|c|}
\hline Nutrition care self-efficacy items & Mean \pm SD & Unconfident & $\begin{array}{l}\text { Neither } \\
\text { confident nor } \\
\text { unconfident }\end{array}$ & Confident \\
\hline $\begin{array}{l}\text { Calculating my patients BMI and WHR based on gender } \\
(\mathrm{n}=197)\end{array}$ & $3.81 \pm 0.83$ & $13(6.6 \%)$ & $41(20.8 \%)$ & $143(72.6 \%)$ \\
\hline $\begin{array}{l}\text { Recommending dietary patterns for patients with } \\
\text { type } 2 \text { diabetes }(n=196)\end{array}$ & $3.26 \pm 0.90$ & $44(22.4 \%)$ & $63(32.1 \%)$ & $89(45.4 \%)$ \\
\hline $\begin{array}{l}\text { Managing severely malnourished children according } \\
\text { WHO guidelines }(n=197)\end{array}$ & $3.18 \pm 1.02$ & $53(26.9 \%)$ & $69(34.5 \%)$ & $75(38.1 \%)$ \\
\hline $\begin{array}{l}\text { Explaining the role of dietary cholesterol and saturated } \\
\text { fat elevating blood lipids to patients }(n=197)\end{array}$ & $3.75 \pm 0.84$ & $15(7.6 \%)$ & $45(22.8 \%)$ & $137(69.5 \%)$ \\
\hline $\begin{array}{l}\text { Providing examples of a serving size of meat or dairy } \\
\text { from the food guide pyramid to patients }(n=196)\end{array}$ & $2.88 \pm 0.98$ & $68(34.7 \%)$ & $73(37.2 \%)$ & $55(28.1 \%)$ \\
\hline $\begin{array}{l}\text { Discussing with lactating mothers the maternal and } \\
\text { infant benefits and anticipated challenges with } \\
\text { breastfeeding }(n=198)\end{array}$ & $3.82 \pm 0.92$ & $19(9.6 \%)$ & $27(13.6 \%)$ & $152(76.8 \%)$ \\
\hline $\begin{array}{l}\text { Discussing with patients the role of omega- } 3 \text { and } \\
\text { omega- } 6 \text { fatty acids in heart health }(n=197)\end{array}$ & $3.19 \pm 1.01$ & $48(24.4 \%)$ & $67(34.0 \%)$ & $82(41.6 \%)$ \\
\hline $\begin{array}{l}\text { Discussing calories per gram of protein, carbohydrates } \\
\text { and fat and their basic metabolic roles with patients } \\
(n=197)\end{array}$ & $3.02 \pm 1.01$ & $64(32.5 \%)$ & $64(32.5 \%)$ & $69(35.0 \%)$ \\
\hline $\begin{array}{l}\text { Providing nutrition education for a patient recently } \\
\text { diagnosed with HIV infection }(n=197)\end{array}$ & $3.09 \pm 1.01$ & $62(31.5 \%)$ & $61(31.0 \%)$ & $74(37.6 \%)$ \\
\hline Total mean $(n=194)$ & $29.88 \pm 5.67$ & & & \\
\hline
\end{tabular}

Response scale: $5=$ Very unconfident; $4=$ unconfident; 3 = neither confident nor unconfident; 4 = confident; $5=$ very confident. Very unconfident and unconfident were combined to yield 'Unconfident' and very confident and confident were combined to yield 'Confident'. Responses do not add up to 207 due to non-response for some of the items.

\section{Types of nutrition education resources that contributed to future doctors' current nutrition-related knowledge, attitudes towards, and self-efficacy in nutrition care}

Reading and self-directed learning were the commonly used strategies to acquire information on nutrition (Figure 1). 


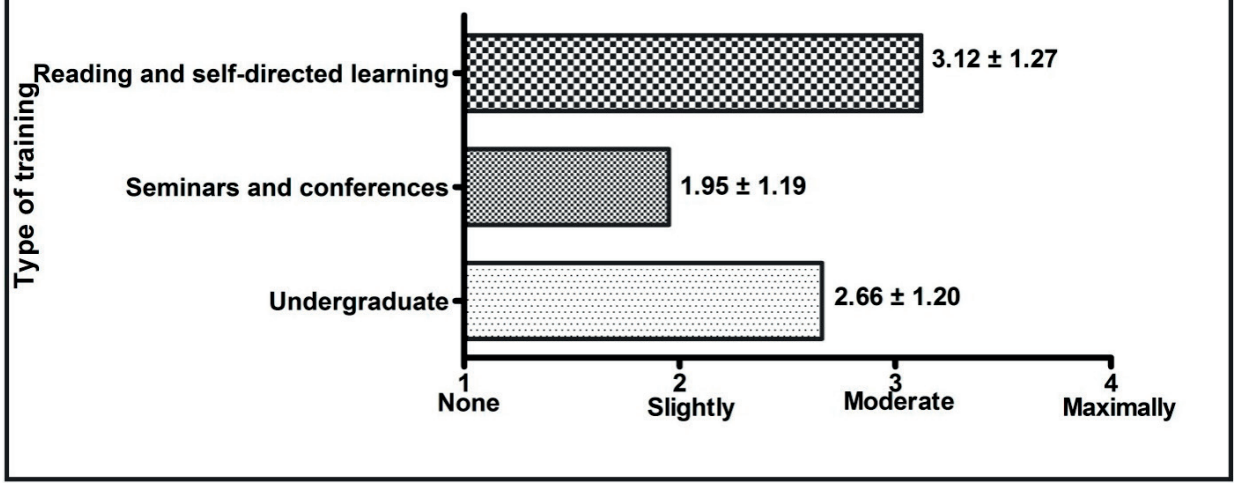

Figure 1: Types of nutrition education that have contributed to the future doctors' current nutrition-related competencies. Students were asked to indicate the extent to which each type of nutrition education had contributed to their current level of nutrition competency on a 4-point Likert scale. $1=$ None, $2=$ Slightly, $3=$ Moderate, and $4=$ Maximal

\section{Association between knowledge, attitudes and self-efficacy}

Future doctors' attitude towards nutrition care correlated positively with their self-efficacy scores $(r=0.371, p<0.001)$. However, their nutrition-related knowledge did not correlate with their attitudes $(r=-0.014, p>0.05)$ and self-efficacy in nutrition care $(r=0.073$, $p>0.05)$.

\section{Discussion}

We evaluated future doctors' nutrition-related knowledge, attitudes and self-efficacy in nutrition care. Associations among these variables were also investigated. Future doctors' nutrition-related knowledge levels were generally good but important knowledge gaps were noted. Their attitudes towards nutrition care were generally positive but they were unsure of their confidence in providing nutrition care to patients. Attitudes toward and self-efficacy in nutrition care correlated positively with each other, but neither correlated with nutrition-related knowledge.

Comparable to previous studies future doctors answered $64 \%$ of all the knowledge items $35,36,45$.

An important finding of this study was that future doctors' nutrition-related knowledge was associated with their level of clinical training. In both the 'malnutrition in children' and 'diabetes and obesity' topics, clinical year three students performed better than clinical year 1 and 2 students. Given the fact that these topics are clinically-oriented, 
clinical year three students might have had greater exposure than clinical year 1 and 2 students.

Conversely, students in clinical year 1 had higher scores than those in clinical year 2 and 3 in the 'nutrients, energy and their deficiencies' topic. This is a basic nutrition concept topic usually taught in preclinical years 1 and 2 . It is thus plausible that as the students' progressed through their training; they might have forgotten some of these basic nutrition concepts learned during the early part of their training. This finding gives credence to previous reports that students may fail to integrate basic concepts of nutrition with its clinical relevance if it is solely taught in the preclinical years of medical school ${ }^{35}$. Nutrition education should thus be integrated as a theme throughout the medical curriculum.

In examining future doctors' scores in the three nutrition topics, we found that their mean scores in the 'malnutrition in children nutrition' topic were the lowest compared to those scored in the other topics assessed. In addition future doctors' self-efficacy in the management of severely malnourished children was also among the lowest reported. This finding is concerning due to the fact that malnutrition is prevalent in Ghana but future doctors have inadequate knowledge in malnutrition in children and also lack confidence in their ability to manage malnourished children. Inadequacies in nutrition education and students being unsatisfied with their current nutrition education in the curriculum as reported previously ${ }^{39}$ may be responsible for future doctors' poor competencies regarding nutrition. A revision of the entire curriculum to identify gaps and avenues to include nutrition topics of this nature is suggested.

In agreement with several studies future doctors' attitudes towards nutrition care

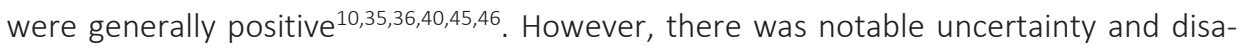
greement regarding the effectiveness of health education in changing patient behaviour. Almost $50 \%$ of the students felt that health education may not be effective in promoting patients' adherence to healthy lifestyles. Future doctors' apparent uncertainty in the effectiveness of health education has also been reported previously ${ }^{10,47}$. Taking note of the fact that health professionals' beliefs in the effectiveness of treatment guidelines could influence their practice behaviour ${ }^{47}$, future doctors may not readily provide nutrition advice to patients at all times if they do not believe in the efficacy of such advice. Patient poor adherence/compliance, provider lack of counselling skills, lack of support systems for providers, and among others could be responsible for future doctors' uncertainty in the effectiveness of health education.

Although the future doctors' attitudes towards nutrition care were positive, same could not be described for their self-efficacy in nutrition care. Out of nine aspects of providing nutrition care in the general practice setting; future doctors were generally confident in their ability to providing nutrition care in only 3 areas but reported being unconfident or uncertain in their ability to provide nutrition care for the rest, suggesting a low level of nutrition self-efficacy. These findings are similar to those reported by Crowley et al among a sample of medical students from New Zealand ${ }^{10}$. 
The relatively low level of self-efficacy in nutrition care could be due to the format of nutrition training they received. Evidence from a previous study among these participants found lectures on selected topics to be the commonest and most frequent format of nutrition instruction ${ }^{39}$. Lectures may be a good way of introducing new concepts to students but lectures alone might not enhance self-efficacy as its opportunities for practical and clinical experiences are limited ${ }^{48}$. In identifying teaching and learning methods that could promote self-efficacy as well as attitudes in nutrition care, Mogre et al ${ }^{34}$ found the following to be important: role modelling by practicing physicians ${ }^{49}$; role playing using either simulated or real patients ${ }^{50-53}$, encouraging clinical bedside nutritional teaching ${ }^{48}$, demonstrations and hands on practice sessions ${ }^{49-52,54-56}$, viewing and discussion of videos and web-based cases ${ }^{51,57}$.

In this study we found that future doctors' attitudes correlated positively with their self-efficacy in nutrition care. Alluding to Bandura's social learning theory ${ }^{58}$; future doctors' feelings of self-efficacy in nutrition care are necessary to promote favourable attitudes and nutrition practice behaviour. Evidence from previous findings found self-efficacy in nutrition care to be associated to nutrition practice behaviour in both medical students and practicing doctors $51,53,54,56,59$.

Self-directed learning being the highest contributor to future doctors' current nutrition-related competencies gives credence to previous findings that medical students value nutrition education and are ready to learn it if the opportunity is granted them $35,46,60$.

\section{Strengths and Limitations}

The findings of this study increase our understanding of the situation of nutrition training in medical education in Ghana and the larger sub-Saharan Africa region. The use of previously validated survey items and the use of nutrition experts to examine the content validity of the survey items enhanced confidence in our findings. Furthermore, using an instrument that is based on items relevant to global nutrition issues may help facilitate the recognition and prioritization of nutrition content in medical education and also makes the findings relevant to other countries in similar nutrition situations like Ghana.

However, our study is not without limitations. Its cross-sectional nature makes it difficult to establish causality. Nonetheless it gives a snapshot of the state of the nutrition competencies of future doctors and the situation of nutrition training in Ghana and probably the rest of sub-Saharan Africa. Our study reports on the nutrition competencies of students from a single medical school. This makes it difficult to generalize our findings. However, given the fact that this is the first study in Ghana as well as in several sub-Saharan African countries to investigate nutrition care competencies of future doctors, our findings serve as a basis and lay the foundation for future research in this subject. Another limitation of this study is the use of self-reports to measure nutrition competence making our findings liable to social desirability. Despite this observation, it is widely accepted that 
self-report measures of nutrition competence could be used as a proxy for actual measure of competence ${ }^{42}$. Findings of self-reports are usually as valid as are more elaborate and expensive tests ${ }^{61}$.

\section{Future research directions}

Future studies should explore factors contributing to the current status of future doctors' nutrition care competencies and nutrition education in general identifying barriers and facilitators. Also, future studies should consider the effects of students' attitudes and selfefficacy on future nutrition practice behaviour and clinical outcomes of patients.

\section{Conclusion}

Future doctors' had favourable attitudes towards nutrition care but had inadequate knowledge in some key nutrition topics. They were also uncertain in their confidence to provide nutrition care in the general practice setting. Enhancing doctors' attitudes and self-efficacy during medical school may be important in improving their nutrition practice behaviour.

\section{Acknowledgements}

Authors wish to thank the students of the University for Development Studies, School of Medicine and Health Sciences for their support and acceptance to take part in the research.

Authors also wish to thank Benjamin Anane-Asamoah of the ICT Directorate of the University for Development Studies for helping with the development of some of the items of the questionnaire.

\section{Conflict of interest statement}

No conflicts of interest.

\section{Funding}

No funding was received. 


\section{References}

1. IDF. Diabetes Atlas 7th Edition. IDF 2015.

2. UNICEF. Improving child nutrition: The achievable imperative for global progress: United Nations Children's Fund; 2013.

3. UNICEF, WHO, World Bank. Levels and trends in child malnutrition: UNICEF-WHO-World Bank joint child malnutrition estimates. New York 2015.

4. UNICEF. Undernutrition contributes to nearly half of all deaths in children under 5 and is widespread in Asia and Africa. 2017; http://data.unicef.org/nutrition/malnutrition.html. Accessed 20/11/2017, 2017.

5. United Nations Children's Fund. Improving Child Nutrition:The achievable imperative for global progress. New York 2013.

6. Ainsworth M. What Can We Learn from Nutrition Impact Evaluations?: Independent Evaluation Group Studies. Washington, D.C: The World Bank; 2010.

7. American Dietetic Association. Nutrition and you: Trends 2008. Report of Results. American Dietetic Association. 2008.

8. American Dietetic Association. Nutrition and you: Trends 2008. Report of Results. 2011.

9. Abramson S, Stein J, Schaufele M, Frates E, Rogan S. Personal exercise habits and counseling practices of primary care physicians: a national survey. Clin J Sport Med 2000;10(1):40-48.

10. Crowley J, Ball L, Han DY, Arroll B, Leveritt M, Wall C. New Zealand Medical Students Have Positive Attitudes and Moderate Confidence in Providing Nutrition Care: A Cross-Sectional Survey. J Biomed Educ 2015;2015:7

11. Mihalynuk TV, Coombs JB, Rosenfeld ME, Scott CS, Knopp RH. Survey correlations: proficiency and adequacy of nutrition training of medical students. J Am Coll Nutr 2008;27(1):59-64.

12. Adams KM, Lindell KC, Kohlmeier M, Zeisel SH. Status of nutrition education in medical schools. Am J Clin Nutr 2006;83(4):941S-944S.

13. Adams KM, Kohlmeier M, Powell M, Zeisel SH. Nutrition in Medicine: Nutrition Education for Medical Students and Residents. Nutr Clin Pract 2010;25(5):471-480.

14. Chung M, van Buul V, Wilms E, Nellessen N, Brouns F. Nutrition education in European medical schools: results of an international survey. Eur J Clin Nutr 2014;68(7):844-846.

15. Gramlich LM, Olstad DL, Nasser R, et al. Medical students' perceptions of nutrition education in Canadian universities. Appl Physiol Nutr Metab 2010;35(3):336-343.

16. Tziraki C, Graubard BI, Manley M, Kosary C, Moler JE, Edwards BK. Effect of Training on Adoption of Cancer Prevention Nutrition-Related Activities by Primary Care Practices: Results of a Randomized, Controlled Study. J Gen Intern Med 2000;15(3):155-162.

17. Glanz K, Gilboy MB. Physicians, preventive care, and applied nutrition: selected literature. Acad Med 1992;67(11):776-781.

18. Krause T, Fox H. Nutritional knowledge and attitudes of physicians. J Am Diet Assoc 1977;70(6):607-609.

19. Soltesz KS, Price JH, Johnson LW, Telljohann SK. Perceptions and practices of family physicians regarding diet and cancer. Am J Preven Med 1994;11(3):197-204.

20. St Jeor ST, Krenkel JA, Plodkowski RA, Veach TL, Tolles RL, Kimmel JH. Medical nutrition: a comprehensive, school-wide curriculum review. Am J Clin Nutr 2006;83(4):963S-967.

21. Bruer RA, Schmidt RE, Chapel T. Nutrition education for physicians: alternative federal roles for creating an improved system: Richard Schmidt Associates; 1993.

22. Walker WA. Innovative teaching strategies for training physicians in clinical nutrition: an overview. J Nutr 2003;133(2):541S-543S.

23. Makowske M, Feinman RD. Nutrition education: a questionnaire for assessment and teaching. Nutr J 2005;4:2.

24. Touger-Decker R. Nutrition education of medical and dental students: innovation through curriculum integration. Am J Clin Nutr 2004;79(2):198-203. 
25. Wong V, Millen BE, Geller AC, Rogers AE, Maury J, Prout MN. What's in store for medical students? Awareness and utilization of expert nutrition guidelines among medical school preceptors. Prev Med 2004;39(4):753-759.

26. Intersociety Professional Nutrition Education Consortium. Bringing physician nutrition specialists into the mainstream: rationale for the Intersociety Professional Nutrition Education Consortium. Am J Clin Nutr 1998;68(4):894-898.

27. Levine BS, Wigren MM, Chapman DS, Kerner J, Bergman R, Rivlin R. A national survey of attitudes and practices of primary-care physicians relating to nutrition: strategies for enhancing the use of clinical nutrition in medical practice. Am J Clin Nutr 1993;57(2):115-119.

28. Kushner RF. Barriers to providing nutrition counseling by physicians: a survey of primary care practitioners. Prev Med 1995;24(6):546-552.

29. Hiddink G, Hautvast J, Van Woerkum C, Fieren C, Van't Hof M. Consumers' expectations about nutrition guidance: the importance of primary care physicians. Am J Clin nutrition. 1997;65(6):1974S-1979S.

30. Kolasa K. Developments and challenges in family practice nutrition education for residents and practicing physicians: an overview of the North American experience. Euro J Clin Nutr 1999;53:S89-S96.

31. Nicholas $L$, Roberts $D C$, Pond $D$. The role of the general practitioner and the dietitian in patient nutrition management. Asia Pac J Clin Nutr 2003;12(1):3-8.

32. Singh $H$, Duerksen DR. Survey of clinical nutrition practices of Canadian gastroenterologists. Can J Gastroenterol Hep 2006;20(8):527-530.

33. Coombs JB, Barrocas A, White JV. Nutrition care of older adults with chronic disease: attitudes and practices of physicians and patients. South Med J 2004;97(6):560-566.

34. Mogre V, Scherpbier AJJA, Stevens F, Aryee P, Cherry MG, Dornan T. A realist synthesis of educational interventions to improve nutrition care competencies and delivery by doctors and other healthcare professionals. BMJ Open 2016:6(10):e010084

35. Vetter ML, Herring SJ, Sood M, Shah NR, Kalet AL. What do resident physicians know about nutrition? An evaluation of attitudes, self-perceived proficiency and knowledge. J Am Coll Nutr 2008;27(2):287-298.

36. Hu SP, Wu, M.Y. \& Liu, J.F. . Nutrition knowledge, attitude and practice among primary care physicians in Taiwan. J Am Coll Nutr. 1997;16(5):439-442.

37. Block JP, DeSalvo KB, Fisher WP. Are physicians equipped to address the obesity epidemic? knowledge and attitudes of internal medicine residents. Prev Med 2003;36(6):669-675.

38. American Dietetic Association. Nutrition and you: trends 2008. American Dietetic Association,. Chicago 2011.

39. Bonevski B, Campbell E, Sanson-Fisher R. Primary care practitioners and health promotion: a review of current practices. Health Promot J Austr 1996;6(1):22.

40. Hyska J, Mersini E, Mone I, et al. Assessment of knowledge, attitudes and practices about public health nutrition among students of the University of Medicine in Tirana, Albania. South East Eur J Public Health 2014;1(2014).

41. McGaghie WC, Van Horn L, Fitzgibbon M, et al. Development of a measure of attitude toward nutrition in patient care. Am J Prev Med 2001;20(1):15-20.

42. Mihalynuk TV, Scott CS, Coombs JB. Self-reported nutrition proficiency is positively correlated with the perceived quality of nutrition training of family physicians in Washington State. Am J Clin Nutr 2003;77(5):1330-1336.

43. Weinsier RL, Boker JR, Brooks CM, et al. Priorities for nutrition content in a medical school curriculum: a national consensus of medical educators. Am J Clin Nutr 1989;50(4):707-712.

44. Wynn K, Trudeau JD, Taunton K, Gowans M, Scott I. Nutrition in primary care Current practices, attitudes, and barriers. Can Fam Physician 2010;56(3):e109-e116.

45. Walsh CO, Ziniel SI, Delichatsios HK, Ludwig DS. Nutrition attitudes and knowledge in medical students after completion of an integrated nutrition curriculum compared to a dedicated nutrition curriculum: a quasi-experimental study. BMC Med Educ 2011;11.

46. Spencer EH, Frank E, Elon LK, Hertzberg VS, Serdula MK, Galuska DA. Predictors of nutrition counseling behaviors and attitudes in US medical students. Am J Clin Nutr 2006;84(3):655-662. 
47. Laws RA, Kirby SE, Davies GPP, et al. Should I and can I? A mixed methods study of clinician beliefs and attitudes in the management of lifestyle risk factors in primary health care. BMC Health Serv Res 2008;8(1):44.

48. Powell-Tuck J, Summerbell C, Holsgrove G, Garrow J. Four years' experience of an undergraduate medical nutrition course. J R Soc Med 1997;90(2):67-72.

49. Carson JAS, Gillham MB, Kirk LM, Reddy ST, Battles JB. Enhancing self-efficacy and patient care with cardiovascular nutrition eduction. Am J Prev Med 2002;23(4):296-302.

50. Hillenbrand KM, Larsen PG. Effect of an educational intervention about breastfeeding on the knowledge, confidence, and behaviors of pediatric resident physicians. Pediatrics 2002;110(5).

51. Ockene JK, Ockene IS, Quirk ME, et al. Physician training for patient-centered nutrition counseling in a lipid intervention trial Pre Med 1995;24(6):563-570.

52. Gance-Cleveland B, Sidora-Arcoleo K, Keesing H, Gottesman MM, Brady M. Changes in nurse practitioners' knowledge and behaviors following brief training on the healthy eating and activity together (HEAT) guidelines. J Pediatr Health Care 2009;23(4):222-230.

53. Conroy MB, Delichatsios HK, Hafler JP, Rigotti NA. Impact of a preventive medicine and nutrition curriculum for medical students. Am J Prev Med 2004;27(1):77-80.

54. Eisenberg DM, Miller AM, McManus K, Burgess J, Bernstein AM. Enhancing medical education to address obesity:"See one. Taste one. Cook one. Teach one."JAMA Intern Med 2013;173(6):470-472.

55. Schlair S, Hanley K, Gillespie C, et al. How medical students' behaviors and attitudes affect the impact of a brief curriculum on nutrition counseling. J Nutr Educ Behav 2012;44(6):653-657.

56. Levy J, Harris J, Darby P, Sacks R, Dumanovsky T, Silver L. The primary care nutrition training programme: An approach to communication on behavior change. Health Promot Pract 2011;12(5):761-768.

57. Carson JAS, Gillham MB, Kirk LM, Reddy ST, Battles JB. Enhancing self-efficacy and patient care with cardiovascular nutrition education. American J Prev Med 2002;23(4):296-302.

58. Bandura A. Self-efficacy: The exercise of control: Macmillan; 1997.

59. Dacey M, Arnstein F, Kennedy MA, Wolfe J, Phillips EM. The impact of lifestyle medicine continuing education on provider knowledge, attitudes, and counseling behaviors. Med Teach 2013;35(5):e1149e1156.

60. Weinsier RL, Boker JR, Feldman EB, Read MS, Brooks CM. Nutrition knowledge of senior medical students: a collaborative study of southeastern medical schools. Am J Clin Nutr 1986;43(6):959-968.

61. Baird LL. Using Self-Reports to Predict Student Performance. Research Monograph No. 7.. College Entrance Examination Board, New York, 1976 


\section{Chapter}

\section{Nutrition care practices, barriers, competencies and education in nutrition: A survey among Ghanaian medical doctors}

Victor Mogre, Fred C.J. Stevens ${ }^{2}$, Paul A. Aryee, Femke L. Matorwmasen-Akkermans, Braimah Abubakari and Albert J.J.A Scherpbier Medical Science Educator 2018; 1-10. DOI: https://doi.org/10.1007/s40670-018-0591-9 


\section{Abstract}

Objective: We evaluated medical doctors' nutrition care practices, competencies and barriers to providing nutrition care. Furthermore, doctors' satisfaction and perceived adequacy of their nutrition education as well as the use and effectiveness of training and learning resources for continuing nutrition education were also investigated.

Design: Cross-sectional

Setting and participants: We included medical doctors working at various levels of care in Ghana who responded to either an online or paper-based survey. Appropriate statistical tools were used to analyse the data.

Results: Majority (70\%) of the 114 doctors who responded to the survey estimated that more than $60 \%$ of their patients needed nutrition care. However, only $\leq 40 \%$ received such care. More than $80 \%$ of doctors referred patients to dieticians/nutritionists. Comfort levels correlated positively $(r=0.288 ; p=0.002)$ with attitudes about nutrition care. The most common barriers to nutrition care were lack of time (79\%); inadequate knowledge (78.6\%) and counselling skills (68.4\%). About 66\% perceived their nutrition education in medical school to be inadequate, and more than $70 \%$ were either unsatisfied or undecided with their nutrition educational experiences. Perceived adequacy $(r=0.200$; $p=0.016)$ and satisfaction with nutrition education ( $r=0.218 ; p=0.002)$ were associated with doctors' comfort levels. Only $30 \%$ were currently using a nutrition-related learning resource for continuing education in nutrition.

Conclusion: Doctors felt their patients required more nutrition care than they could provide. Their nutrition care was hindered by lack of time, inadequate knowledge, confidence and counselling skills. Educational interventions that improve on medical doctors' attitudes and comfort levels in providing nutrition care may be needed.

Key words: Nutrition care, Medical doctors, Barriers, Nutrition education, Ghana 


\section{Introduction}

Nutrition or diet-related diseases are important contributors to the global burden of morbidity and mortality. For instance, excessive weight has been shown to be a risk factor for cardiovascular disease, type 2 diabetes mellitus, hypertension, osteoarthritis, cancers among others ${ }^{1-3}$. Referred to as non-communicable or chronic diseases (NCD), these conditions are responsible for $70 \%$ of all deaths annually, making them by far the leading cause of death in the world ${ }^{4}$. Eighty-two percent of these deaths occur in low-and middle-income countries. Furthermore, three of the four major risk factors contributing to the rise of NCDs are nutrition-related: physical inactivity, unhealthy diets and harmful use of alcohol ${ }^{5}$. In comparison with pharmacological interventions to patients, there is increasing evidence that the benefits of nutrition, diet and physical activity interventions are either comparable or better, with attended benefits of low side effects, reduced risk and lower cost ${ }^{6-8}$.

In the pursuit of optimal health, doctors are important players who are best positioned to encourage individuals to adopt healthy lifestyles such as optimal dietary intake and physical activity. However, many medical doctors are unable to effectively provide nutrition counselling due to inadequate training, resulting in inadequate knowledge, skills and low confidence to provide nutrition care ${ }^{9-14}$. These may stem from factors such as low priority given to nutrition education during medical school, inadequate faculty to provide nutrition training, resistance to the addition of new courses or lectures in nutrition, with a curriculum emphasizing on disease treatment rather than disease prevention ${ }^{15-22}$. Obviously, it is necessary to develop and integrate nutrition education into the training of medical doctors to realise its translational impact on prevention and treatment of disease $^{23}$.

Recognising the fact that doctors drive the health care system in Ghana and in several countries within the sub-Saharan Africa region and considering that nutrition related conditions are mostly presented at the health facilities, nutrition education for doctors is of utmost importance. Increasing greater awareness and support for nutrition care among doctors may help them become proponents and important sources of referral to dieticians, nutritionists and other relevant healthcare professionals ${ }^{23}$.

The majority of studies that report on medical doctors' nutrition care practices, competencies and barriers as well as their nutrition educational experiences during medical school emanate from developed countries such as the US, Australia, Canada, New Zealand and the UK ${ }^{10,11,23-30}$. The evidence regarding this subject matter is limited in subsaharan Africa. In our search of the literature we found only two studies from sub-saharan africa which reported on medical students' views and perceptions of their nutrition education ${ }^{13,14}$ but not those of medical doctors. It is not usually possible to translate these findings to the context of sub-Saharan Africa that is characterised by poor healthcare systems, nutrition transition, and urbanisation. It is thus necessary to undertake a needs assessment of the nutrition care competencies, barriers and views of medical doctors 
regarding their past nutrition education (during medical school) as they are important stakeholders of the medical education milieu. A recent realist review ${ }^{31}$ has noted the importance of executing needs assessments to inform the design of educational interventions to improve nutrition educational experiences during medical school or continuing education in nutrition during practice.

The current study aims to answer the following research questions?

1. What are doctors' nutrition care practices and competencies (comfort levels and attitudes) in the general practice setting?

2. How do doctors' perceive barriers to the provision of nutrition care in the general practice setting?

3. Do doctors feel adequately prepared by and satisfied with their nutrition educational experiences during medical school?

4. Are doctors' nutrition care practices and competencies related to their perception of the adequacy and satisfaction of their nutrition educational experiences during medical school?

5. How do doctors perceive the effectiveness of the nutrition-related training and learning resources they are currently using or may use in the future?

\section{Methods}

\section{Participants}

This cross-sectional study included medical doctors across Ghana working in varied clinical settings. This study is part of a series intended to investigate the nutrition educational needs of both students and medical doctors. The current study was conducted from October 2016 to July 2017. Participants were approached through two procedures: face-toface and an online survey. Through purposive sampling doctors were approached at their practices by one of the authors and invited to participate in the study. Those who agreed and consented were given a printed version of the questionnaire to fill out at their convenience. This procedure yielded 26 responses. For the online survey procedure, the questionnaire was mailed through a survey link to all listed members of the Ghana Medical Association (GMA), which is an organised group of all medical practitioners. The link of the online survey was also placed on social media groups (Facebook and Whatsup) whose members were mainly doctors working at a particular hospital or graduates of the same medical school. Non-respondents were sent three reminders at: 1 month; 3 months and 4 months respectively after the initial mailing. Ethical approval for the study was granted by the Ethical approval for this study was granted by the Navrongo Health Research Centre Institutional Review Board (Ethics Approval ID: NHRCIRB209), Ghana 


\section{The survey instrument}

The survey instrument consisted mostly of closed ended items with a few open ended. The items were adapted from previous surveys conducted among doctors ${ }^{25,29,32}$ and medical students ${ }^{13,33}$. The items measured the following variables.

a. Nutrition care practice: This was measured by two items. The first item requested doctors to indicate the proportion of their patients they believed will benefit from nutrition care. The second item required doctors to indicate the proportion of their patients they believed actually received nutrition care from them.

b. Referral of patients to dieticians/nutritionists and reasons for referring patients: Doctors' referral behaviour was assessed using the question "Do you refer your patients to dieticians? Yes or No". To determine the frequency of referrals doctors were asked to indicate the number of times they referred their patients to a dietician/nutritionist in the last 12 months. Using open ended questions doctors were asked to provide reasons why they usually referred their patients to dieticians/nutritionist and the kind of conditions they did so.

c. Comfort levels in providing nutrition care: Based on a 10-point Likert scale doctors were asked to indicate their level of comfort in discussing nutrition topics with their patients categorised into 3 broad areas: general nutrition; maternal and child nutrition, and nutrition in chronic disease. This scale consisted of 11 items categorised into three broad areas. Items for this scale were adapted from previous study conducted among Canadian family physicians.

d. Attitudes towards nutrition care: Consisting of 10 statements relating to nutrition care, doctors were asked to indicate their level of agreement with those statements using a 5-point Likert scale (1=totally disagree; 2 =disagree; 3 =neutral; 4=agree and 5=totally agree). Items for this scale were derived from the Nutrition attitudes survey.

e. Barriers to the provision of nutrition care: Using a list of seven barriers to nutrition care, doctors were asked to indicate the extent to which they considered those barriers hindered them from providing effective nutrition care to their patients using a 5-point Likert Scale (1=totally disagree; 2=disagree; 3=neutral; 4=agree and 5=totally agree).

f. Satisfaction: Based on four items adapted from previous surveys ${ }^{10,14,33}$, doctors were asked to indicate their level of satisfaction regarding the quality and quantity of nutrition education they received during medical school using a 5-point Likert scale ( $1=$ very dissatisfied; 2 = Dissatisfied; 3 = neither satisfied nor dissatisfied; 4 = satisfied and 5 = very satisfied).

g. Training adequacy: Using a 3-point Likert scale doctors were asked to indicate their perceived level of adequacy of the nutrition training they received during medical school $^{24}$ ( 1 = inadequate; $2=$ adequate and $3=$ more than adequate). 
h. Nutrition-related learning/training resources for continuing education in nutrition: Using items from a previous survey ${ }^{25}$, doctors were asked to indicate the nutrition-related learning/training resources they considered to be effective in improving their continuing nutrition education ( $1=$ not effective; 2 =somewhat ineffective; $3=$ neither ineffective nor effective; 4=effective 5=very effective). In addition, doctors were asked to indicate which nutrition-related learning/training resources they were currently using or are likely to use in the future for their continuing nutrition education.

i. Demographics: These included gender; age; medical school graduated; number of years since graduation from medical school; place of practice and type of practice (i.e. house officer, medical officer, consultant/specialist, etc).

\section{Statistical analysis}

All data were entered into and analysed using the statistical software IBM SPSS version 21.0. Descriptive statistics of mean, standard deviation, frequencies and bar charts were used to describe the data. All categorical variables were compared using cross-tabulation with Chi-square. Comparisons between categorical and continuous variables were done using student t-test and one-way ANOVA, where appropriate. Pearson zero-correlations were calculated to identify relationship among continuous variables. A p-value of less than 0.05 at a $95 \%$ confidence interval was considered statistically significant. Composite "comfort" and "attitude" scores were generated for each doctor. These continuous variables were calculated by averaging each doctor's responses to the individual component variable.

\section{Results}

\section{General characteristics of participants}

One hundred and fourteen medical doctors responded to the questionnaire in which 99 answered all items to yield a completion rate of $87 \%$. Reasons for non-response included lack of time and poor internet service at practice location of some participants. Demographic and general characteristics of the doctors are presented in Table 1. The doctors had a mean (SD) age of 36.70 (10.41) years and have been working for an average of 10 years. 
Table 1: General and demographic characteristics of participants

\begin{tabular}{|c|c|}
\hline Variable & Frequency (\%) \\
\hline \multicolumn{2}{|l|}{ Gender } \\
\hline Male & $63(66.3 \%)$ \\
\hline Female & $32(33.7 \%)$ \\
\hline \multicolumn{2}{|l|}{ Type of practice } \\
\hline House officer & $16(16.7 \%)$ \\
\hline Medical officer & $41(42.7 \%)$ \\
\hline Consultant/Specialist & $31(33.3 \%)$ \\
\hline Resident & $8(8.3 \%)$ \\
\hline \multicolumn{2}{|l|}{ Type of place of practice } \\
\hline Teaching Hospital & $48(47.5 \%)$ \\
\hline Regional Hospital & $7(6.9 \%)$ \\
\hline District Hospital & $30(29.7 \%)$ \\
\hline Public Clinic & $5(5.0 \%)$ \\
\hline Private Hospital & $11(10.9 \%)$ \\
\hline \multicolumn{2}{|l|}{ Medical school graduated } \\
\hline KNUST-SMS & $24(26.4 \%)$ \\
\hline UDS-SMHS & $25(27.5 \%)$ \\
\hline UGMS & $29(31.9 \%)$ \\
\hline Foreign trained & $13(14.3 \%)$ \\
\hline \multicolumn{2}{|l|}{ Age } \\
\hline$<35$ years & $48(57.1 \%)$ \\
\hline$\geq 35$ years & $36(42.9 \%)$ \\
\hline \multicolumn{2}{|c|}{ Number of years since graduating from medical school } \\
\hline$\leq 10$ years & $63(64.5 \%)$ \\
\hline$>10$ years & $29(31.5 \%)$ \\
\hline
\end{tabular}

Frequencies do not add up to 99 due to missing responses.

Doctors' comfort levels in discussing nutrition topics with patients and attitudes towards nutrition care

With a Cronbach alpha value of 0.92 and explaining 75\% of the total variance three factors emerged from the 11-item comfort scale. Having factor loadings that ranged from $0.60-0.80$, items in factor 1 ( 4 items) constituted maternal and child nutrition. Items on factor 2 (factor loadings $=0.52-0.83$ ) with 3 items related to general nutrition and wellbeing and factor 3 (factor loadings $=0.59-0.78$ ) constituted nutrition in chronic disease. All three factors had alpha values that ranged from $0.77-0.87$. Doctors reported being more comfortable discussing items relating to the maternal and child nutrition factor (7.84; $95 \% \mathrm{Cl}: 5.40-6.26 ; p<0.001)$ compared with those of the general nutrition $(5.83$ $95 \% \mathrm{Cl}: 5.40-6.26, p<0.001)$ and with nutrition in chronic disease factors $(6.70 ; 95 \% \mathrm{Cl}$ : $7.45-8.23)$. Among responses of individual doctors to the items, strong correlations 
$(r=787 ; p<0.001)$ were noted, with those who were comfortable discussing one topic being generally comfortable with all topics. Doctors who reported being more comfortable discussing nutrition topics with their patients were more likely to be older $(p=0.015)$ and graduated from medical school in the last $\geq 10$ years $(p=0.029)$.

A Cronbach alpha value of 0.81 was recorded for the 6-item attitude towards nutrition care scale. With a total mean (SD) weighted attitude score of $4.2(0.76)(95 \% \mathrm{Cl}: 4.12-$ 4.41 ), over $80 \%$ of doctors agreed that nutrition care was one of their responsibilities; $94 \%$ felt nutrition is a significant component in the prevention and progression of chronic diseases; $81 \%$ felt their patients needed more nutrition information than they were able to provide and $84 \%$ believed that their patients will adopt healthier lifestyle if counselled to do so. Attitude scores correlated positively with comfort levels of participants $(r=0.288$, $=0.002$ ) but not with number of years since graduation from medical school. Furthermore $p$, attitude scores did not differ significantly by type of practice ( $p=0.571$ vs $p=0.685$ ), medical school graduated ( $p=0.067$ vs $p=0.125)$, gender ( $p=0.768$ vs $p=0.149)$ and type of place of practice ( $p=0.406$ vs 0.249 )

\section{Practice of nutrition care}

Some $86 \%(n=86)$ of the participants said nutrition care was relevant to their practice, $12.1 \%(n=12)$ said irrelevant and $2.0 \%(n=2)$ were undecided. As shown in Figure 1, a large number $(76.4 \%, n=87)$ of doctors believed that more than $60 \%$ of their patients will benefit from nutrition care; however majority of them felt only $\leq 40 \%$ of their patients actually received such counselling $(p<0.001)$. Poor agreement was observed between proportion of patients doctors believed will benefit from nutrition care compared with proportion of those they said were actually receiving such care (Kappa $=0.023, S . E=0.035, p=0.502$ ). Only $27 \%$ ( $n=27$ ) of doctors spent $\geq 6$ minutes providing nutrition care to their patients. Perceived relevancy of nutrition care was not associated with doctor's estimate of the proportion of patients who will benefit from nutrition care but was significantly $(p=0.012)$ associated with their estimate of proportion of patients who actually received such care. Doctors who were more comfortable discussing nutrition topics with patients believed a greater proportion of their patients would benefit from nutrition counselling $(p<0.001)$ and were more likely to estimate that a greater proportion of their patients actually received such counselling $(p=0.011)$. Doctors' estimate of the proportion of patients that they believed will benefit from nutrition care differed by their attitudes towards nutrition care $(p=0.002)$. However, their estimate of the proportion of patients that actually received nutrition care was independent of their attitudes towards nutrition care $(p=0.527)$. 


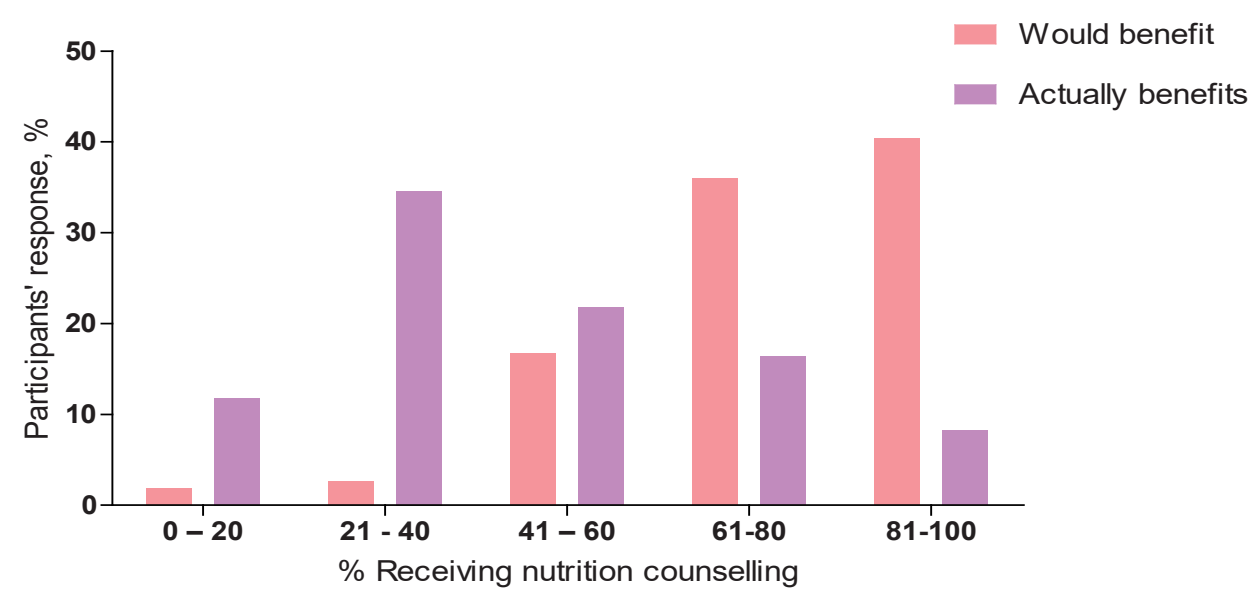

Figure 1: Participants' estimates of the proportion of their patients they believed would benefit from nutrition care and the proportion of patients actually receiving such counselling

\section{Referral to dieticians/nutritionist}

More than three-quarters $(84.8 \%, n=95)$ of doctors referred patients to dieticians. Fiftyseven percent $(n=49)$ reported making fewer than 10 referrals; $18.6 \%(n=16)$ made 10 20 referrals and $24.4 \%(n=21)$ made $>20$ referrals in the last 12 months. Frequency of referrals did not differ by the proportion of patients doctors believed will benefit from nutrition care $(p=0.726)$ or with the proportion of patients actually receiving nutrition care $(p=0.527)$ or with the amount of time spent providing nutrition care $(p=0.358)$. In addition, frequency of referrals was not associated with type of practice ( $p=0.379)$; medical school graduated ( $p=0.089)$; number of years since graduating from medical school $(p=0.830)$; doctor's age $(p=0.956)$; gender $(p=0.643)$; doctor's place of practice $(p=0.274)$ and attitudes towards nutrition care $(p=0.450)$. Doctors who were more comfortable discussing nutrition with their patients were more likely to refer patients to dieticians/nutritionists for specialist care $(p=0.032)$. The number of times doctors referred patients to dieticians was independent ( $p=0.840$ ) of their level of comfort when discussing nutrition topics with patients or with their attitudes towards nutrition care $(p=0.532)$.

Doctors referred patients to dieticians for varied medical conditions including general nutrition $(7.1 \%, n=7)$, malnutrition $(37.8 \%, n=37)$, and chronic conditions such as diabetes (55.1\%, $n=54)$.

Doctors had several and varied reasons for referring patients to dieticians. Doctors said they referred patients as a result of high patient load as illuminated in the following code "Too many patients to attend to" (Participant 10, Male Medical Officer).

Another reason for referral was to allow the patients to obtain specialist care as demonstrated in the following code "Provide some advice but prefer expert opinion since management of cases is a shared responsibility" (Participant 14, Male Senior Specialist). 
Other reasons provided by the doctors were: availability of nutritionists/dieticians to refer patients to, to help motivate patient and reinforce advice given and inadequate knowledge and skills to provide nutrition care. These reasons are substantiated by the following codes.

"There is a nutritionist available who would do a better job." (Participant 101, Male Medical Officer)

"I do counsel, but refer them for reinforcement."(Participant 89, Female Specialist)

"I am not well-versed in the detailed caloric contents of our local foods." (Participant 88, Female Resident)

\section{Doctors' reported barriers to the provision of nutrition care}

Commonly perceived barriers were lack of time, doctor's inadequate knowledge about nutrition and inadequate counselling skills (Shown in Figure 2).

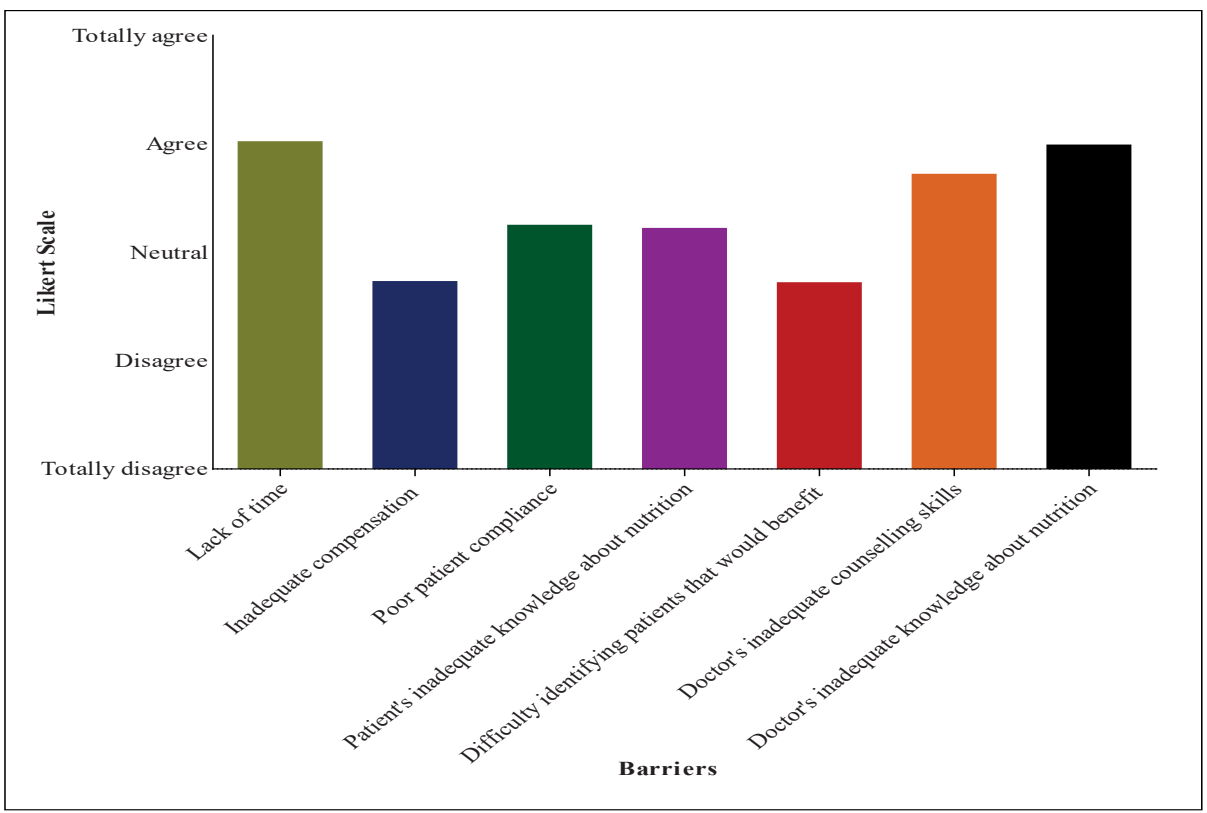

Figure 2: Barriers to providing nutrition care in the general practice setting

\section{Nutrition training during medical school}

About $66 \%(n=66)$ of the doctors said they had inadequate nutrition education during medical school whereas 33.3\% ( $n=33)$ and $1.0 \%(n=1)$ said it was adequate and more than adequate respectively. This finding was independent of gender $(p=0.178)$, age $(r=0.147$; 
$p=0.107)$, number of years since graduation $(r=0.075 ; p=0.476)$, type $(p=0.085)$ and place of practice $(p=0.289)$. More than $70 \%$ of the participants were either unsatisfied or undecided regarding the quality and quantity of their nutrition educational experiences during medical school (Shown in Table 2). These findings did not differ by gender $(p=0.082)$, age $(r=0.149 ; p=0.055)$, number of years since graduation ( $r=0.172 ; p=0.100)$, type $(p=0.059)$ and place ( $p=0.305)$ of practice. Adequacy of nutrition education and satisfaction with nutrition educational experiences correlated positively $(r=0.561 ; p<0.001)$. Notably, doctors who graduated from foreign medical schools were more likely $(p=0.023)$ than their local counterparts to be more satisfied with their nutrition educational experiences during medical school and also felt more adequately prepared from their nutrition training $(p=0.036)$. Doctors who said their nutrition education was adequate $(r=0.200$; $p=0.016)$, and those who felt more satisfied with their nutrition educational experiences during medical school $(r=0.218 ; p=0.002)$ were more likely to report being more comfortable discussing nutrition topics with their patients. However, the perceived adequacy of, and satisfaction with nutrition education experiences were not associated with doctors estimate of the proportion of their patients they believed will benefit from nutrition care ( $p=0.864)$ or proportion of their patients that actually received such care $(p=0.784)$. Doctors who perceived their nutrition education to be inadequate significantly reported spending 2 minutes or less counselling patients about nutrition (15.3\% vs. $10.0 \%$; $p=0.009)$.

Table 2: Doctors' level of satisfaction with their nutrition educational experiences during medical school

\begin{tabular}{|c|c|c|c|c|}
\hline Nutrition educational experiences & Dissatisfied & Neutral & Satisfied & $\begin{array}{l}\text { Weighted } \\
\text { average }\end{array}$ \\
\hline Amount of time dedicated for nutrition & $46(46.9 \%)$ & $20(20.4 \%)$ & $32(32.6 \%)$ & 2.71 \\
\hline Integration of nutrition content into courses/blocks & $43(43.9 \%)$ & $19(19.4 \%)$ & $36(36.8 \%)$ & 2.86 \\
\hline $\begin{array}{l}\text { Inclusion of materials to promote independent study of } \\
\text { nutrition }\end{array}$ & $49(50.0 \%)$ & 19(19.4\%) & $40(31.6 \%)$ & 2.74 \\
\hline Nutrition course content in general & $49(50.0 \%)$ & $21(21.4 \%)$ & $28(18.6 \%)$ & 2.73 \\
\hline
\end{tabular}

Responses for dissatisfied and somewhat dissatisfied were summed to yield dissatisfied. Responses for satisfied and very satisfied were summed to generate satisfied. Frequencies do not add to 99 due to missing responses

\section{Nutrition-related training and learning resources for continuing education in nutrition}

Regarding training types that had contributed to doctors' current nutrition care competencies; reading and self-directed learning had the largest contribution (46.4\%) followed by clinical practice (32.3\%) and undergraduate medical curriculum (25.3\%). Conferences/workshops had the least contribution (12.1\%). Doctors were asked to indicate the effectiveness of a number of nutrition learning resources in improving their continuing 
education in nutrition. The participants said all the listed nutrition learning/training resources could be effective in improving their nutrition counselling skills and did not consider one modality to be more effective than the other. Considering the use of those nutrition-related learning resources, only $41 \%(n=55)$ were currently using any of them (shown in Figure 1). Among those that were using, 43\% ( $n=17)$ were currently calling nutritionists or dieticians for consultation. Although less than half of the participants were currently using a nutrition-related learning/training resource, more than $80 \%$ of them said they were likely to use them in future (shown in figure 3 ).

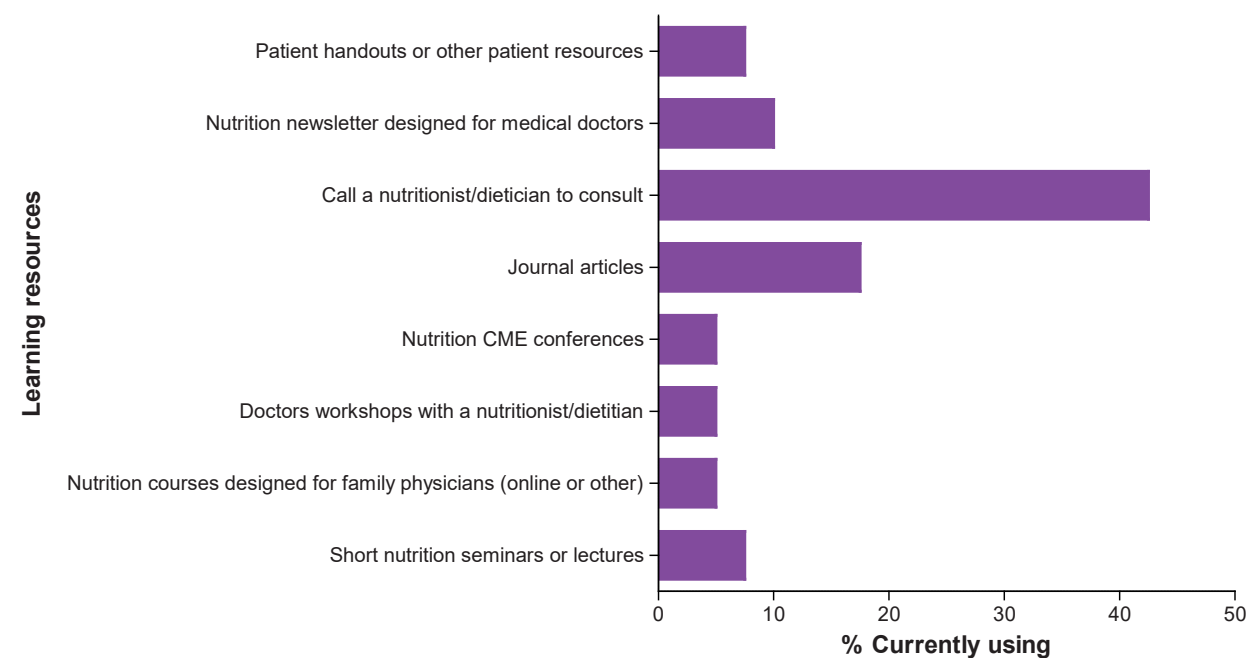

Figure 3: The proportion of respondents indicating that they were currently using the listed resources; some respondents were using more than one resource.

\section{Discussion}

In this study we evaluated doctors' nutrition care competencies, practices and barriers to nutrition care. In addition nutrition education during medical school and continuing education in nutrition were also investigated. Doctors' comfort levels were varied but they had positive attitudes and believed they had an important role to play in nutrition care. They believed a greater majority of their patients will benefit from nutrition care than they could effectively provide. Lack of time, inadequate knowledge and counselling skills were the most common barriers to nutrition care. The doctors were unsatisfied with their nutrition educational experiences in medical school and felt inadequately trained to provide nutrition care. Very few were currently using nutrition-related learning resources although a large majority of them felt that those resources could be effective in improving their nutrition care competencies. 
As shown in previous reports ${ }^{25,34}$ the majority of doctors estimated that a greater proportion of their patients will benefit from nutrition care, however they were unable to provide such care given that they estimated a fewer proportion of their patients actually received the needed nutrition care. A number of barriers were identified for this situation including lack of time, inadequate knowledge in nutrition care and poor nutrition care skills ${ }^{11,25}$.

Corroborating with previous reports 25,34 , almost all doctors referred patients to dieticians for further nutrition care. This may suggest that doctors recognised the benefits of multidisciplinary team-based approach to the provision of patient care and readily supported it. In addition they may also be aware of their limitations regarding nutrition care and recognise the need to grant their patients the opportunity to seek for specialised nutrition care. These assertions are further illuminated by the reasons given by doctors for referring their patients to dieticians/nutritionist in which majority of them said they referred patients to give them the opportunity to receive expert and specialised care in nutrition. It is however worth noting that doctors' frequency of referring patients to dieticians did not correspond with the number of patients they believed will benefit from nutrition care. Consequently several patients may be needing nutrition care but are not referred as needed. Future studies should explore why doctors may not be referring patients to dieticians/dieticians as patients may need it. Given that doctors recognise the role of dieticians/nutritionist in the provision of nutrition care, encouraging doctors to refer patients to these healthcare professionals may improve the provision of nutrition care to patients and subsequently leading to improved clinical outcomes.

Doctors showed moderate comfort levels in their ability to discuss nutrition topics with their patients. Previous reports have noted doctors' ambivalent confidence and selfefficacy in the provision of nutrition care $25,26,34,35$. Taking cognisance of the important role of these in improving doctors' nutrition practice behaviour it is imperative to design educational interventions that can improve the comfort/self-efficacy/confidence levels of doctors. These interventions should be implemented during medical school training and as continuing nutrition education programmes for doctors already in practice.

Reassuringly, doctors had positive attitudes towards the importance and role of nutrition in patient care to improve clinical outcomes. These findings are corroborated by previous reports among doctors ${ }^{25,26,34,35}$ and medical students ${ }^{13,30,33}$.

Attitude and self-efficacy (referred to as comfort levels) that were found to be positively correlated in this study are important determinants of nutrition practice behaviour. It is thus unsurprising that doctors who felt more comfortable discussing nutrition topics with their patients were more likely to estimate that a greater majority of their patients will benefit from nutrition care; more likely to provide nutrition care to their patients and frequently referred patients to dieticians. Furthermore those who had positive attitudes towards nutrition care were likely to estimate a greater proportion of their patients needed nutrition care. This is consistent with those reported by Wynn et al among primary care physicians in Canada ${ }^{25}$ and gives credence to the findings of a recent realist 
review by Mogre et al ${ }^{31}$ in which they reported that improving self-efficacy (measured using comfort levels) may result in improved nutrition practice behaviour. The same review added that building doctors' attitude and empathy for nutrition care may be more important in determining nutrition practice behaviour than increasing doctors' knowledge only.

Another important finding of this study was that number of years after graduation correlated positively with the comfort level of doctors as those who graduated more than 10 years ago were more likely to report being more comfortable discussing nutrition topics with their patients compared to those who graduated in the recent 10 years. Given that majority of doctors indicated that clinical practice moderately contributed to their current nutrition care competence it may suggests that longer duration of patient care which relates to greater number of years after graduation may have exposed doctors to nutrition issues, granted them opportunities to participate in continuing nutrition education workshops and to learn more about nutrition.

Despite the doctors' recognition of nutrition care as being highly relevant to their practice as well as recognising it as their responsibility, majority of them were unsatisfied with their nutrition educational experiences during medical school and felt they had been inadequately trained for nutrition care. These findings, together with those of previous studies ${ }^{10,14,25}$ recognise the need to identify nutrition education that is deemed essential by medical doctors. As shown previously and in the current study, doctors' perception of being inadequately trained in nutrition may demotivate them from effectively providing nutrition care to their expectant patients.

Only $41 \%$ of doctors were currently using any nutrition-related learning resource for continuing nutrition education. These findings are similar to those reported among family physicians in Canada ${ }^{25}$. It is however strange and concerning that these doctors reportedly considered nutrition care to be highly relevant to their practice, felt inadequately prepared for nutrition care but were not currently using any learning/training resources for their continuing education in nutrition. Future studies should explore reasons for doctors' apparent poor interest in using nutrition-related learning/training resources although they report being inadequately trained.

Notwithstanding the fact that a greater majority of the doctors were not currently using any nutrition-related learning/training resource they however considered all the strategies to be effective and were more likely to use them in the future for their continuing education in nutrition-related topics. This is similar to findings reported by Wynn et al ${ }^{25}$ but contrary to previous reports in which doctors have considered patient pamphlets and reading of peer reviewed nutrition journals to be more useful ${ }^{36,37}$.

It is important to note that among the few doctors that were currently using a nutrition-related learning/training resource for their continuing education in nutrition, majority of them consulted their nutritionists/dietician to seek further information or clarification regarding nutrition care of their patients. This finding is consistent with those reported by Wynn et al ${ }^{25}$ among Canadian family physicians and Mihalynuk et al among 
residents in Washington ${ }^{37}$. This is a demonstration of the multidisciplinary approach needed for nutrition care and the doctors' recognition of the important role of the dietician/nutritionist in patient care.

Consistent with previous studies perceived adequacy and satisfaction with nutrition education were significantly associated with doctors comfort levels when discussing nutrition topics with their patients $10,24,38$.

Our finding that doctors from foreign trained medical schools felt more satisfied with their nutrition educational experiences and adequately prepared in nutrition than their Ghanaian trained counterparts presents yet another important reason for the revision and identification of avenues for the inclusion of nutrition education into the medical curricula in Ghana.

\section{Limitations, strengths and future research directions}

It is imperative to note the limitations of this study. The self-report nature of the questionnaire might have influenced the responses of the doctors. However, the self-critical responses provided by the doctors might have minimised the effect of this limitation. The response was low and the participants may be those who have interest in nutrition which might not be representative of the general population of medical doctors in Ghana. This limitation is not peculiar to the current study as previous studies among similar populations have reported low responses to surveys evaluating nutrition practice, education and competencies $34,37,39$. In addition, the effect of this limitation may be minimal given that a high completion rate of the survey items were recorded and also the findings are consistent with those of previous studies from the same setting that had higher responses rates ${ }^{13,14}$. The cross-sectional nature of the study makes it difficult to establish causality. Notwithstanding the limitations, this study has important strengths worth reporting. It is the first study in sub-Saharan Africa to evaluate doctors' nutrition care practice behaviour, comfort levels, attitudes and barriers to nutrition care as well as the views and perceptions of doctors regarding their nutrition education in medical school. It thus makes available data that increases our understanding of the situation of nutrition education in medical education in the sub-region. Furthermore, its findings provide evidence that will serve as foundation towards identifying and defining the nutrition education and training needs of the local medical workforce. The use of standardised and previously validated survey items have helped to increase the credibility of our findings. This study also adopted innovative non-traditional approaches such as social media to reach out to the study participants. The varied responses provided by these doctors may represent a general observation of the nutrition education needs of Ghanaian doctors and thus granting avenues for future studies to plan appropriately. This is particularly more relevant given that further research is required to determine doctors' priorities for nutrition care in relation with other aspects of patient care. The impact of attitudes and confidence on the 


\section{Chapter 5}

actual nutrition care behaviour of doctors and its ultimate impact on the clinical outcomes of patients warrant further research. Appropriate nutrition education during medical school training and during practice is needed to allow the development of skills and confidence to support patients to make healthy dietary choices and help prevent chronic diseases.

\section{Conclusion}

Doctors believed their patients required more nutrition care than they could provide. However, they were hindered by factors such as lack of time, inadequate knowledge, confidence and counselling skills. Doctors' referral to dieticians/nutritionist may be important in improving patient nutrition care and clinical outcomes. The inadequacy in nutrition education may be a nationwide problem but not an issue of a single medical school and requires attention and collaboration from all medical schools together with the Ghana National Accreditation Board and the Medical and Dental Council of Ghana. Furthermore, educational interventions that improve on medical doctors' attitudes and comfort levels in providing nutrition care are urgently needed. 


\section{References}

1. Guh DP, Zhang W, Bansback N, Amarsi Z, Birmingham CL, Anis AH. The incidence of co-morbidities related to obesity and overweight: a systematic review and meta-analysis. BMC Public Health 2009;9(1):88.

2. US Department of Health Human Services. The Surgeon General's call to action to prevent and decrease overweight and obesity: Rockville, MD: US Department of Health and Human Services, Public Health Service, Office of the Surgeon General; 2001.

3. Billington CJ, Epstein LH, Goodwin NJ, et al. Overweight, obesity, and health risk. Arch Intern Med 2000;160(7):898-904.

4. WHO. Major NCDs and their risk factors. Noncommunicable diseases 2017; http://www.who.int/ncds/ introduction/en/. Accessed 8/9/2017, 2017.

5. World Health Organization. Global status report on noncommunicable diseases 2014. Geneva: World Health Organization; 2014

6. Estruch R, Ros E, Salas-Salvadó J, et al. Primary prevention of cardiovascular disease with a Mediterranean diet. New Engl J Med 2013;368(14):1279-1290.

7. Sacks FM, Svetkey LP, Vollmer WM, et al. Effects on blood pressure of reduced dietary sodium and the Dietary Approaches to Stop Hypertension (DASH) diet. New Engl J Med 2001;344(1):3-10.

8. Look AHEAD Research Group. Cardiovascular effects of intensive lifestyle intervention in type 2 diabetes. N Engl J Med 2013;2013(369):145-154.

9. Schucker B, Wittes JT, Cutler JA, et al. Change in physician perspective on cholesterol and heart disease: results from two national surveys. JAMA 1987;258(24):3521-3526.

10. Mihalynuk TV, Scott CS, Coombs JB. Self-reported nutrition proficiency is positively correlated with the perceived quality of nutrition training of family physicians in Washington State. Am J Clin Nutr 2003;77(5):1330-1336.

11. Kushner RF. Barriers to providing nutrition counseling by physicians: a survey of primary care practitioners. Preventive medicine. 1995;24(6):546-552.

12. Adams KM, Kohlmeier M, Powell M, Zeisel SH. Nutrition in medicine: nutrition education for medical students and residents. Nutr Clin Pract 2010;25(5):471-480.

13. Mogre V, Aryee PA, Stevens FC, Scherpbier AJA. Future Doctors' Nutrition-Related Knowledge, Attitudes and Self-Efficacy Regarding Nutrition Care in the General Practice Setting: A Cross-Sectional Survey. Med Sci Educ 2017:1-8.

14. Bonevski B, Campbell E, Sanson-Fisher R. Primary care practitioners and health promotion: a review of current practices. Health Promot J Austr1996;6(1):22.

15. St Jeor ST, Krenkel JA, Plodkowski RA, Veach TL, Tolles RL, Kimmel JH. Medical nutrition: a comprehensive, school-wide curriculum review. Am J Clin Nutr 2006;83(4):963S-967.

16. Bruer RA, Schmidt RE, Chapel T. Nutrition education for physicians: alternative federal roles for creating an improved system: Richard Schmidt Associates; 1993.

17. Walker WA. Innovative teaching strategies for training physicians in clinical nutrition: an overview. J Nutr 2003;133(2):541S-543S.

18. Makowske M, Feinman RD. Nutrition education: a questionnaire for assessment and teaching. Nutr J 2005;4:2

19. Touger-Decker R. Nutrition education of medical and dental students: innovation through curriculum integration. Am J Clin Nutr 2004;79(2):198-203.

20. Wong $V$, Millen BE, Geller AC, Rogers AE, Maury J, Prout MN. What's in store for medical students? Awareness and utilization of expert nutrition guidelines among medical school preceptors. Prev Med 2004;39(4):753-759.

21. Intersociety Professional Nutrition Education Consortium. Bringing physician nutrition specialists into the mainstream: rationale for the Intersociety Professional Nutrition Education Consortium. Am J Clin Nutr. 1998;68(4):894-898. 
22. Touger-Decker R. Nutrition education of medical and dental students: innovation through curriculum integration. Am J Clin Nutr 2004;79(2):198-203.

23. Kris-Etherton PM, Akabas SR, Bales CW, et al. The need to advance nutrition education in the training of health care professionals and recommended research to evaluate implementation and effectiveness. Am J Clin Nutr 2014;99(5):1153S-1166S.

24. Mihalynuk TV, Coombs JB, Rosenfeld ME, Scott CS, Knopp RH. Survey correlations: Proficiency and adequacy of nutrition training of medical students. J Am Coll Nutr 2008;27(1):59-64.

25. Wynn K, Trudeau JD, Taunton K, Gowans M, Scott I. Nutrition in primary care Current practices, attitudes, and barriers. Can Fam Physician 2010;56(3):e109-e116.

26. Crowley J, Ball L, Han DY, et al. Doctors' attitudes and confidence towards providing nutrition care in practice: Comparison of New Zealand medical students, general practice registrars and general practitioners. J Prim Health Care 2015;7(3):244-250.

27. Kolasa KM, Rickett K. Barriers to providing nutrition counseling cited by physicians: a survey of primary care practitioners. Nutr Clin Pract 2010;25(5):502-509.

28. Smith S, Seeholzer EL, Gullett H, et al. Primary care residents' knowledge, attitudes, self-efficacy, and perceived professional norms regarding obesity, nutrition, and physical activity counseling. J Grad Med Educ 2015;7(3):388-394.

29. Vetter ML, Herring SJ, Sood M, Shah NR, Kalet AL. What do resident physicians know about nutrition? An evaluation of attitudes, self-perceived proficiency and knowledge. J Am Coll Nutr 2008;27(2):287-298.

30. Crowley J, Ball L, Han DY, Arroll B, Leveritt M, Wall C. New Zealand medical students have positive attitudes and moderate confidence in providing nutrition care: a cross-sectional survey. J Biomed Educ 2015;2015:7

31. Mogre V, Scherpbier AJ, Stevens F, Aryee P, Cherry MG, Dornan T. Realist synthesis of educational interventions to improve nutrition care competencies and delivery by doctors and other healthcare professionals. BMJ Open 2016;6(10):e010084.

32. McGaghie WC, Van Horn L, Fitzgibbon M, et al. Development of a measure of attitude toward nutrition in patient care. Am J Prev Med 2001;20(1):15-20.

33. Hyska J, Mersini E, Mone I, et al. Assessment of knowledge, attitudes and practices about public health nutrition among students of the University of Medicine in Tirana, Albania. South East Euro J Public Health. $2015 ; 1(1)$.

34. Nowson CA, O'Connell SL. Nutrition knowledge, attitudes, and confidence of Australian general practice registrars. J Biomed Educ 2015;2015:6

35. Ray S, Rajput-Ray M, Ball L, et al. Confidence and attitudes of doctors and dietitians towards nutrition care and nutrition advocacy for hospital patients in Kolkata, India. J Biomed Educ 2015;2015:6

36. Kelly A, Joffres M. A survey of physicians' uses/opinions regarding nutrition education resources. J Can Diet Assoc 1990;51(3):409-412.

37. Mihalynuk TV, Knopp RH, Scott CS, Coombs JB. Physician informational needs in providing nutritional guidance to patients. Fam Med 2004;36(10):722-726.

38. Spencer EH, Frank E, Elon LK, Hertzberg VS, Serdula MK, Galuska DA. Predictors of nutrition counseling behaviors and attitudes in US medical students. Am J Clin Nutr 2006;84(3):655-662.

39. Hicks K, Murano P. Physician Perspectives on Nutrition Counseling and Nutrition Focused Continuing Medical Education in Texas. Soc Med 2016;10(3):99-105. 


\title{
Chapter
}

\section{Why nutrition education is inadequate in the medical curriculum:}

A qualitative study of students' perspectives on barriers and strategies

\author{
Victor Mogre, Fred C.J. Stevens, Paul A. Aryee, Anthony Amalba, \\ and Albert J.J.A Scherpbier
} BMC Medical Education 2018; 18(1):26. DOI: 10.1186/s12909-018-1130-5. 


\section{Abstract}

Background: The provision of nutrition care by doctors is important in promoting healthy dietary habits, and such interventions can lead to reductions in disease morbidity, mortality, and medical costs. However, medical students and doctors report inadequate nutrition education and preparedness during their training at school. Previous studies investigating the inadequacy of nutrition education have not sufficiently evaluated the perspectives of students. In this study, students' perspectives on doctors' role in nutrition care, perceived barriers, and strategies to improve nutrition educational experiences are explored.

Methods: A total of 23 undergraduate clinical level medical students at the $5^{\text {th }}$ to final year in the School of Medicine and Health Sciences of the University for Development Studies in Ghana were purposefully selected to participate in semi-structured individual interviews. Students expressed their opinions and experiences regarding the inadequacy of nutrition education in the curriculum. Each interview was audio-recorded and later transcribed verbatim. Using the constant comparison method, key themes were identified from the data and analysis was done simultaneously with data collection.

Results: Students opined that doctors have an important role to play in providing nutrition care to their patients. However, they felt their nutrition education was inadequate due to lack of priority for nutrition education, lack of faculty to provide nutrition education, poor application of nutrition science to clinical practice and poor collaboration with nutrition professionals. Students opined that their nutrition educational experiences will be improved if the following strategies were implemented: adoption of innovative teaching and learning strategies, early and comprehensive incorporation of nutrition as a theme throughout the curriculum, increasing awareness on the importance of nutrition education, reviewing and revision of the curriculum to incorporate nutrition, and involving nutrition/dietician specialists in medical education.

Conclusion: Though students considered nutrition care as an important role for doctors, they felt incapacitated by non-prioritisation of nutrition education, lack of faculty for teaching of nutrition education, poor application of nutrition science and poor collaboration with nutrition professionals. Incorporation of nutrition as a theme in medical education, improving collaboration, advocacy and creating enabling environments for nutrition education could address some of the barriers to nutrition education.

Key words: Nutrition education, Medical students, Barriers, Strategies, Curriculum, Qualitative research 


\section{Background}

There is ample evidence that nutrition interventions can decrease morbidity, mortality, human suffering, and medical costs ${ }^{1-4}$. Given the cost-effectiveness of disease prevention through nutrition over treatment, the availability of practicing doctors with adequate knowledge, attitudes and skills in nutrition is very essential ${ }^{5}$. Studies have reported that if doctors gave nutrition advice to their patients the incidence of nutrition-related diseases will decline ${ }^{6,7}$. However, most doctors usually miss the opportunity to provide nutrition care to patients in the general practice setting and often refer patients to hospital dieticians or nutritionists, that is if these are available ${ }^{8,9}$. Research suggests that practicing doctors lack sufficient nutrition care competencies to provide dietary advice to their patients ${ }^{10-15}$.

Indeed, there is an unfilled role for medical education in preparing doctors to provide nutrition care to patients ${ }^{16}$. Several studies investigating the situation of nutrition education in the medical curriculum have found both practicing doctors and medical students reporting inadequate nutrition education during medical school ${ }^{14,17-21}$.

Several barriers to inadequate nutrition education have been reported largely in the form of symposiums, commentaries, essays and special articles ${ }^{8,22-25}$. Few studies have systematically investigated the issue ${ }^{5,26-28}$. These studies generally echo the perspectives of teachers or practicing doctors. A number of strategies to improving nutrition education in the medical curriculum have also been discussed by some of these studies ${ }^{8,22,23}$. However, the perspectives of students are unclear.

This is an important issue to investigate, as students are likely to bring unique and valuable perspectives on the inadequacy of nutrition education in their curriculum. Their views should be considered in efforts to improve nutrition education in the medical curriculum.

The study therefore aimed to explore students' perceptions of doctors' role in nutrition care and barriers that prevent effective nutrition education and strategies that could potentially increase the effectiveness of nutrition education in the medical curriculum. The following research questions were investigated

1. What are the roles of doctors in the provision of nutrition care in the general practice setting?

2. Why is nutrition education inadequate in the medical curriculum?

3. How could nutrition education be improved in the medical curriculum? 


\section{Methods}

\section{Theoretical orientation}

Our research was informed by two closely related theories ${ }^{29}$ : social learning theory and social cognitive theory. Arguably, delivery of nutrition care by health care providers is a behaviour demonstrated in the social context of the workplace settings which is influenced by observing and modelling the behaviours, attitudes, and emotional reactions of others (e.g. superiors) ${ }^{30}$. Also embedded in our study is the social cognitive theory of learning which emphasizes how cognitive, behavioural, personal, and environmental factors interact to determine motivation and behaviour ${ }^{31}$. Nutrition education in the medical school setting is a learning process that is influenced by factors within the academic environment relating to the quantity, quality and nutrition content in the curriculum as well as the teaching and learning methods employed and the reinforcement experienced by the individual and by others.

\section{Methodology}

Our research is within the constructivists' research paradigm. In constructivism, knowledge is socially constructed and realities are generated by the interaction of social, cultural and interpersonal factors ${ }^{32,33}$. Accordingly, there are multiple accounts of realities and meaning is realised through mutual interactions between the researcher and participants and the context of the research ${ }^{34}$. In this research approach, identification of the background and assumptions of the researcher and how they may influence the data collection and analysis is important and should be reflected upon by the researcher and shared with readers to facilitate interpretation of the research findings ${ }^{35,36}$. VM has a nutrition background and teaches in the medical school. His co-authors have significant experience in medical education (AJJA, FCJS), qualitative research (AJJA, FCJS), nutrition (PAA), pharmacology (AA) and also teach in the medical school (AJJA, FCJS, AA, PAA).

\section{Setting, participants and procedures}

We adopted a purposive sampling to select the study participants. All participants were selected from the University for Development Studies, School of Medicine and Health Sciences (UDS-SMHS), which runs a problem-based undergraduate medical curriculum. In the first three years, students are taken through the basic sciences using system-based blocks. The next year focuses on the learning of pathology and patho-physiology of diseases. Although students have some early patient encounters, the first four years are usually non-clinical (preclinical). In preparing students to qualify as doctors, the remaining three years focus on the clinical application of principles learned in the preclinical years and the creation of opportunities for students to learn from real patients at the hospital 
through departmental clinical rotations. The UDS-SMHS also runs a community-based education and service (COBES) programme in which students live, learn and provide service in rural communities of Ghana for a period of 4 weeks per year during year 2 to 4 . It is during the preclinical years that majority of nutrition education is learned.

Our participants were therefore clinical level students, who had covered over $50 \%$ of the curriculum. Our choice of participants and sampling procedure was informed by our aim to select participants who will provide rich information ${ }^{36,37}$ regarding nutrition education in the medical curriculum.

VM contacted all prospective participants through face-to-face meetings after usual lectures. During such meetings, the purpose of the study was explained and participants were also informed that the interview will be audio-recorded. They were assured of the confidentiality of the recordings. Participation was voluntary and both written and verbal informed consent was obtained from all participants who agreed to participate in the study. The institutional review board of the Navrongo Health Research Institute approved the research protocol.

\section{Semi-structured interviews}

All data was collected using semi-structured individual interviews. Semi-structured interviews are frequently used in healthcare and provide the researcher the opportunity to have guidance on areas to explore while allowing for some flexibility to enable discovery or elaboration ${ }^{38,39}$. All interviews were conducted by VM to ensure uniformity. Interviews were informed by open ended questions to explore values, attitudes, experiences, opinions, and behaviours of participants regarding nutrition education in the medical curriculum (See Appendix for interview guide). Interviews provided time and scope for participants to give detail and in depth about their opinions regarding nutrition education in the medical curriculum, allowing for their understanding and point of view rather than assuming generalisations. The questions were derived from the literature ${ }^{27,40}$. During the interviews VM probed and sought for clarification or elaboration of participants' responses as needed. Opportunities were provided for participants to voice out unsolicited opinions and experiences.

The questions were evaluated by all members of the team who have varying levels of experience in qualitative research, medical education and nutrition. We pilot tested the semi-structured interviews on a group of 8 students to ensure clarity and understanding of the questions. During this process participants provided feedback regarding the structure of the questions and also identified areas that needed clarification. AA observed these pilot interview sessions, providing the opportunity to train and give feedback. The data generated from the pilot sessions were not included into the main data analysis. 


\section{Data analysis}

All interviews were transcribed verbatim without identifying information by a trained research assistant, and entered into MAXQDA (version 12), a qualitative data analysis software. All subsequent data analyses were conducted using a comparative strategy ${ }^{41,42}$. Coding was performed by VM, who initially applied as many codes as needed for each transcript and inductively renamed, reorganised and redefined codes as required within categories. Another member of the team, AA went through the same process to evaluate VM's categorisation. VM and AA then compared and discussed their findings until a consensus was achieved. Disagreements were resolved through discussions and adjudication by AA or FCJS if needed. To raise the analytic level from categorical to conceptual, axial coding was performed to identify dominating themes and to explore relationship among these themes. All members of the research team discussed the results of the analysis process until a consensus was established. Data collection and analysis were conducted simultaneously until thematic saturation was realised.

\section{Results}

Twenty-three students participated in the semi-structured interviews lasting 10-20 minutes each during the study period, July to September, 2015. This number was arrived at after the point of saturation and further data collection and/or analysis was unnecessary. Table 1 presents the demographic characteristics of the participants.

Table 1: Demographic characteristics of participants $(n=23)$

\begin{tabular}{lcl}
\hline Variable & Frequency & $\%$ \\
\hline Mean Age (SD) & $25.0(2.76)$ & \\
Age category & & 61 \\
Below 25 & 14 & 39 \\
$25+$ & 9 & \\
Gender & & 61 \\
Male & 14 & 39 \\
Female & 9 & \\
Clinical year & & 30 \\
Year one & 7 & 39 \\
$\quad$ Year two & 9 & 30 \\
$\quad$ Year three & 7 & \\
\hline
\end{tabular}

Four main themes emerged from the data: role of doctors in nutrition care, adequacy of nutrition education, barriers to nutrition education, and strategies to increase and improve nutrition education. Illustrative quotes are provided to substantiate each of these themes. Statements represent the views of students and not the authors. 
Theme 1: The role of doctors and nutrition care

The majority of the students felt that doctors had an important role to play in providing nutrition care to patients although they recognised that nutritionists and dieticians were trained to perform such functions. They considered the role of the doctor to be one that is supportive or complementary to that of the nutritionist/dietician. The main roles identified by the students are shown in list 1.

\section{List 1: Roles doctors could play in nutrition care}

- Nutrition advice/education

- Supporting patients to follow healthful diets

- Collaborating with nutritionists/dieticians

- Monitoring progress of nutrition care

- Nutrition diagnosis

- Referring patients to nutritionists

- Advocating for nutrition care

"They [doctors] should also play a part in the health education of the patient. So when they see a patient of any kind they have to advise them on their nutrition, diet and other behaviours that affect their nutrition and all of that."

Students believed that the doctor should be the first to provide basic nutrition care and refer the patient to a nutritionist/dietician for specialised care if need be.

"When they [patients] come to the hospital, the doctor has the first encounter with them. The doctor could begin to provide nutrition care based on his/her minimal knowledge in nutrition. Then afterwards if he/she sees that there is still more to be done, then he/she can refer the patient to the nutritionist or the dietician who are specially trained to provide nutrition care."

In order to assess whether medical students considered themselves to be responsible for the nutrition care of their patients when they become doctors, they were asked a hypothetical question about how they will react if a diabetic patient sought for dietary advice from them in the general practice setting. All of the students said they will feel obligated and enthusiastic to provide such dietary advice to the patient.

"Oh I will feel happy to assist the patient. As such I will tell the patient the causes of diabetes and what to do to avoid [prevent] it. I will advise the patient to avoid excessive intake of sugar."

Although students felt that nutrition care was important and felt obligated to provide it to patients, majority of them felt unconfident in their ability to provide such care to their patients. 
"Well I think it is the right thing to find out how they [patients] manage their nutrition based on their condition. But looking at the kind of training that we [students] also have, you realize that much attention is not given to nutrition. So if a patient should ask me at this moment about nutritional status or let's say about their nutritional situation, I might not feel adequate enough to answer."

Theme 2: Adequacy of nutrition education

Although students felt that nutrition care was important and felt obligated to provide it to patients, majority of them felt unconfident in their ability to provide such care to their patients.

"Well I think it is the right thing to find out how they [patients] manage their nutrition based on their condition. But looking at the kind of training that we [students] also have, you realize that much attention is not given to nutrition. So if a patient should ask me at this moment about nutritional status or let's say about their nutritional situation, I might not feel adequate enough to answer."

In addition, almost all the students considered the quality and quantity of their nutrition education to be inadequate.

"For me I think... even though we have done something very little concerning nutrition, it is as if we [students] have not done anything. We [students] have been given very little training when it comes to nutrition."

It is thus unsurprising that students felt they required further education in nutrition although they were in the senior years of their medical education.

"I think there is more to be done because we [students] have not really had more lectures on it. We [students] have not been taken through a lot on nutrition; it is just a little bit of everything like small, small, small. So I think it would be better if we [students] are given more education on nutrition before we are able to come out."

In order to gain more knowledge and skills in nutrition some students undertook selfdirected learning and also consulted nutrition departments at the hospital on their own volition to acquire knowledge and skills in nutrition care.

"Because we [students] realized that our nutrition education was inadequate, when we [students] came here we had to go to the nutrition department to kind of like talk to them to take us through how they managed their [nutrition department] malnutrition cases" 


\section{Theme 3: Barriers to adequate nutrition education}

Students identified a number of barriers contributing to the inadequacy in their nutrition education. These barriers were classified into three subthemes: personal, interpersonal and environmental barriers as shown in Table 2. In accordance with the descriptions of Williams et al. ${ }^{43}$, personal barriers refers to factors related to the individual, whiles interpersonal barriers are attributable to relationship between two or more individuals. Contextual factors that may influence nutrition education are described as environmental barriers. Next we describe further some of these barriers.

Table 2: Barriers to nutrition education generated from the data

\begin{tabular}{ll}
\hline Category & Barrier \\
\hline Personal & Poor translation of nutrition science to clinical practice \\
& Perception that nutrition care is not the responsibility of doctors \\
& Faculty's lack of knowledge in clinical nutrition \\
& Faculty's lack of motivation to teach nutrition \\
Interpersonal & Poor collaboration with nutrition professionals in medical education \\
Environmental & Lack of priority for nutrition \\
& Lack of faculty to provide nutrition education \\
& Poor integration of nutrition as a theme throughout the curriculum \\
& Already overcrowded curriculum \\
& Time constraints \\
& Lack of role models to model nutrition care
\end{tabular}

Poor translation of nutrition science to clinical practice

Most students felt that the application of nutrition science to clinical practice was poor in most of the already limited nutrition lectures they received, especially during their preclinical training. The apparent lack of application of nutrition science to clinical practice made it difficult for them to appreciate the relationship between nutrition and health and to apply nutrition during their clinical training.

"Though at the preclinical level we [students] were given a little bit knowledge about nutrition it was not practical. It was like kind of raw information. You come here [clinical training] then you realize that there is no correlation. Not correlation as such but here [clinical training] you realize that it demands you to apply."

Another student adds that "the practical aspect of nutrition education has not really been taught or we are not exposed to it" 
Perception that nutrition care is not the responsibility of doctors

Another important barrier identified by the students was the perception of faculty and curricula planners that nutrition care may not be the responsibility of doctors and as such did not see the need to include adequate content of nutrition education into the curriculum. According to the students, faculty and curricula planners considered nutrition care to be the role of nutritionists and thus the medical curriculum should concentrate on how to treat diseases.

"May be the authorities' feel it's not an essential part of medical training. I don't know but that is the way I see it"

"May be the focus of the whole medical training is more on treating diseases."

"I think because there are nutritionist who are specially trained to provide nutrition care"

\section{Poor collaboration with nutrition professionals in medical education}

Students were concerned that their nutrition education did not involve nutrition professionals thus presenting limited opportunities to collaborate to learn about nutrition. The poor collaboration made it difficult for them to appreciate the role of nutrition care in improving the clinical outcomes of patients and also do not foster inter-professional training to promote multidisciplinary care required in patient care.

"If there can be more integration. For instance when we [students] have cases that are nutrition related, it is still being managed by medical professionals and doctors. I mean once in a while dieticians come but it is like off record or something. So if those who are directly involved in the nutrition sector can be integrated more. May be it will help. But right now they are separated, nutrition is on one side and medicine is on the other. So maybe if there is a problem that will be the area it is."

Students identified that nutrition professional were not involved in their training because they were not available i.e. they were in short supply.

\section{Lack of priority for nutrition education}

Majority of the students felt that the medical curriculum concentrates largely on anatomy, physiology and pathology with a little attention for nutrition. The students felt strongly that nutrition has not been given the needed attention and priority that it deserved.

"Like we have anatomy, physiology and the other basic sciences incorporated, we don't actually have a course on nutrition incorporated into the courses that we do. I think that is the major issue". 
"It's also because nutrition is not a priority in the medical programme and hence have been given less attention."

According to the students, the lack of priority for nutrition education has resulted in the limited contact hours allocated for nutrition, inadequate content for nutrition in the medical curriculum and poor integration of nutrition education as a theme throughout the entire medical curriculum.

\section{Lack of faculty to teach nutrition}

Students were also concerned about the lack of trained faculty to provide nutrition education.

"May be it could also be that we don't have the people around to actually, I mean teach us or take us through nutrition."

"I think sometimes the lecturers may not be available to give a lecture on that [nutrition]."

A number of students also felt that faculty does not have the needed knowledge and skills to provide students with the required nutrition education.

"May be I think it is.... Because those taking you, they themselves they don't have the clinical knowledge. It is like they just read raw nutrition [theoretical aspect of nutrition] and that's how they also passed it on to you."

\section{Theme 4: Strategies to improve nutrition education}

Having recognized the inadequacy of their nutrition education and the contributing barriers, students provided several important and insightful recommendations that could improve nutrition education.

Increase lectures, tutorials and self-study time for nutrition education

To increase the contact hours and nutrition content in the curriculum students suggested that more lecture topics on nutrition should be included into the curriculum.

"Not really as in a full course but may be some few lectures in nutrition. I don't think we need so much but some few things that you need on the job that to help you, that is very important. I think that aspect could be incorporated into the training, it will help."

Others also suggested that nutrition training could be improved by incorporating nutrition topics into their tutorials to promote self-directed learning. 
"I think that we can also introduce them into our tutorial topics because sometimes for most of the students tutorials really help. You read a lot before you come so you can introduce some of them into our tutorial topics".

Concerning the promotion of self-directed learning and to cater for the overcrowded curriculum, students suggested that articles on nutrition could be given to them to read to promote their understanding of nutrition concepts. The self-study time for students could also be increased to grant students the opportunity to read further on nutrition. In addition, students suggested the organization of symposiums on nutrition in which research papers in nutrition could be presented in order to increase their interest and understanding of nutrition.

"Well it's very dicey because there is time issue and stuff. But if we could get articles or make symposiums where we can teach nutrition courses and make it lively because nutrition is kind of like difficult. So if we make it motivational they will be able to come out and get the knowledge about nutrition so that they can educate the people."

Early incorporation of nutrition as a theme and throughout the entire curriculum

As evidenced by the following code, students felt that their knowledge and skills in nutrition could be improved if nutrition as a theme was incorporated early in their training and maintained throughout the entire curriculum.

"Looking at the early [preclinical] aspect of the training, they can incorporate nutrition subjects there, nutritional modules or topics and things like that especially at the very early aspects of it. So that people [students] will take interest in that and probably might grow their knowledge as they go along the PBL training."

Increasing awareness on the importance of nutrition education in the medical curriculum

Some students opined that nutrition education will be improved if those in charge were educated on the importance and need for nutrition education. Students believed that if faculty and curriculum planners could be convinced of the fact that nutrition is an important role of the doctor, this might change their perception regarding nutrition and increase attention and priority for nutrition. An increase in attention and priority for nutrition education may result in an increase in the content and contact hours for nutrition.

"I think if they can realize that it is also very very important then they can increase the amount of time that is spent on that [nutrition education]". 
Reviewing and revision of the curriculum to incorporate nutrition

Students believed that for nutrition education to be improved there is the need for faculty and curriculum planners to review the entire curriculum to identify students' nutrition educational needs and how and where those needs could be met and incorporated into the curriculum. Students recognised that such revisions could not be effected during their time in the medical school but could be beneficial to those in the succeeding years in medical school.

"That is what I previously stated that they should revise our curriculum and find a place to put nutrition so that at least we [students] will have knowledge about it. At least at this level I should have had enough knowledge about nutrition. So if they put it there those following us can benefit. Right now if they can add it to our curriculum, I think it will be enough."

\section{Involving nutrition/dietician specialists}

Students opined that nutritionists or dieticians should be actively involved in their training. This should take the following forms:

a. Clinical sessions in which nutritionists/dieticians will present nutrition cases

b. Opportunities to consult nutritionist/dieticians for relevant nutrition topics/content

c. Including the nutrition department into clinical rotations

d. Seminars and lectures presented by nutritionists/dieticians

"It [nutrition education] should be added to the medical school's curriculum and we [students] should actually...ehmm if they have a nutrition department, we should actually be exposed to nutrition" "Even in the hospital we [students] can have nutritionist being involved in our training on the ward especially when we [students] are managing patients so that we can have more time with the nutritionist".

\section{Discussion}

General discussion and doctors' roles in nutrition care

Undergraduate clinical level medical students believed medical doctors have a responsibility to provide nutrition care to their patients in the general practice setting. They believe doctor's role regarding nutrition care should be one of identifying patients in need of nutrition care, providing first line nutrition management, advocating nutrition care for patients, referring patients to nutritionists/dieticians for specialist care and reinforcing nutrition care provided by nutritionists/dieticians. These students felt their current nutri- 
tion education was inadequate due to barriers such as lack of priority for nutrition education, poor application of nutrition to clinical practice, an already overcrowded medical curriculum, and lack of faculty trained in nutrition and poor collaboration with nutrition professionals in the teaching and learning of nutrition. Students recognised the need to increase awareness on the relevance and need for nutrition education in the medical curriculum among faculty and curriculum planners and to undertake a revision of the medical curriculum to identify nutrition educational needs and avenues to meet such needs as well as recognising the need to involve nutritionists/dieticians in their training.

Students' views that medical doctors have an important role to play regarding nutrition care are similar to those expressed by medical educators, practicing doctors ${ }^{27,44}$ and students ${ }^{45}$ of previous studies from other parts of the world.

\section{Barriers to nutrition education and suggested strategies}

Consistent with previous findings, students were concerned that their nutrition education was inadequate ${ }^{17,45-50}$.

Although an already overcrowded curriculum and inadequate contact hours/content for nutrition education were frequently described as barriers to effective nutrition education, some of the students believed that this was more an issue of low priority and attention for nutrition education. According to these students if nutrition was considered an important responsibility of the medical doctor, faculty and curriculum planners will find avenues within the curriculum to include nutrition. There is thus the need to increase faculty and curriculum planners' awareness on the relevance and need for nutrition education in the medical curriculum.

Students' suggestion of integrating nutrition as a theme throughout the entire curriculum to cater for barriers such as inadequate contact hours/content for nutrition and overcrowded curriculum is consistent with best practices for improving nutrition education ${ }^{5,51}$. Integration of nutrition as a theme in the medical curriculum has been shown to be effective in reducing students' perception of the inadequacy of the amount of nutrition taught to them and improving nutrition clinical skills as assessed by Objectively Structured Clinical Examination (OSCE) scores ${ }^{52}$. The integration of nutrition as a theme throughout the curriculum should be comprehensive including the early stages of preclinical training up to clinical training and continued to postgraduate medical education 5,8,24,53.

Fundamental to the feasibility of integrating nutrition as a theme throughout the medical curriculum is the need to undertake a review of the entire curriculum. This provides opportunities to look into the formal and informal/hidden curriculum to identify gaps and avenues to integrate nutrition education and improve the learning environment 5,54.

Given that multidisciplinary teaching is a key focus of education guidelines for future health professionals ${ }^{55}$ it is consistent that students recognised poor collaboration with 
nutrition and other health professionals in their nutrition education as an important barrier. The apparent poor collaboration may not give students the opportunity to realise the multidisciplinary nature of nutrition care ${ }^{56}$ and will hinder inter-professional development and collaboration required for clinical care ${ }^{57}$. The identification and integration of nutrition content into the curriculum may also be hampered if nutrition professionals are not involved in the planning and development of the medical curricula. Collaborating with nutrition and other relevant health professionals has been found to be effective in improving the status of nutrition education in the medical curriculum ${ }^{56}$. Reflecting on the success of a nutrition education initiative at the University of Cambridge, Ball et al ${ }^{56}$ asserts that the multidisciplinary nature of the programme contributed to its success. The multidisciplinary team included medical practitioners, dieticians, nutritionists, and nurses in the delivery and evaluation of nutrition education sessions. This multidisciplinary nature was recognized at two levels: during the development of the intervention and implementation of the teaching and learning strategies of the intervention. This approach has also been widely encouraged by other institutions to model the contribution of health professionals in addressing nutrition in patient practice ${ }^{58,59}$.

The students suggestion of providing them with nutrition articles and the organisation of nutrition symposiums and seminars to address the barriers of overcrowded and inadequate curriculum are widely acceptable innovative teaching and learning strategies of improving nutrition education ${ }^{60,61}$. These strategies are more likely to be adopted by students and could result in improved nutrition education given that they perceive nutrition to be important to their future practice as medical doctors.

The lack of faculty for nutrition education is an important barrier that has been identified in previous findings $5,8,24$. Closely linked to this barrier is faculty's lack of interest in nutrition education. These barriers are fundamental to the effectiveness of nutrition education in the sense that if faculty trained in nutrition are lacking, increases in contact hours and nutrition content and integration of nutrition as a theme in the curriculum may not yield the needed results. The availability of faculty trained in nutrition may facilitate identification of appropriate nutrition content, coordination of teaching and learning activities in nutrition, promotion of active participation in nutrition education activities by both faculty and students, translation of nutrition science to clinical practice, and promotion of role modelling of nutrition care at the hospital ${ }^{52,61-64}$.

\section{Strengths and limitations}

This is the first study to qualitatively explore students' perceptions of nutrition education, barriers and strategies as far as we know. It adds to the existing literature that nutrition education is inadequate and several barriers are contributing to the current situation. Also, the findings identify a number of strategies that could be relied upon to improve nutrition education. An important novelty of the methods of this study is the reliance on 
students' perspectives that hitherto may not be considered as obvious sources of information regarding this topic. The students provided important barriers and strategies that are comparable to those reported by medical educators and practicing doctors. It is yet additional evidence supporting the widely accepted recognition of students as important stakeholders of the curriculum who could be relied upon to improve the learning environment.

This study is not without limitations. Our adoption of non-probability and convenience sampling may result in the recruitment of students with an interest in nutrition, generating a potential source of sampling bias. However, this potential bias can be described as a strength rather than a limitation in qualitative research that propagates for the collection of data from individuals considered as rich sources of information ${ }^{27}$. We recruited students from only one medical school, thus limiting the generalizability of our findings. The opinions expressed by these students, only grant one aspect of the situation of nutrition education in the medical curriculum and may be insufficient in providing a holistic and complete understanding of the situation. Nevertheless, following a constructivists approach, that aims to develop a comprehensive understanding of the situation of nutrition education, their opinions are valuable insights. As a measure to reduce interviewer' bias that may influence students' opinions during the semi-structured interviews, we limited the interviews to questions and clarifications, where necessary and avoided expression of interviewer opinion. Our study explored students' opinions regarding nutrition education but did not evaluate whether improving nutrition education will result in improved nutrition practice behaviour or enhance clinical outcomes.

\section{Implications and future research}

The findings of this study provide data that could inform the planning and design of educational interventions to improve nutrition education. Future studies should investigate whether the removal of the identified barriers could result in improved nutrition education. It will be important for studies to evaluate the effectiveness of the strategies suggested by the students to improve nutrition education in the medical curriculum. In addition future studies should investigate the influence of improved nutrition education on nutrition practice behaviour in the clinical setting.

\section{Conclusion}

Students perceived nutrition care to be an important role and responsibility of medical doctors. They considered their current nutrition education to be inadequate due to personal, interpersonal and environmental barriers. A number of important strategies have been suggested by these students including incorporation of nutrition as a theme in the 
medical curriculum, collaboration, and advocacy and creating enabling environments for nutrition education.

\section{Interview guide}

Medical students

Age

Gender

What is your level in medical school?

In your opinion, what is the role of doctors in providing nutrition care in the general practice setting?

In your opinion, which professionals have a role in giving nutrition care to patients? Probe further for reasons.

How would you feel if a patient suffering from diabetes asked you for nutrition advice in the general practice setting?

Could you tell me your experience of patients requesting for nutrition advice in the general practice?

In what ways might patient care be affected if all doctors were able to give nutrition advice for routine care?

How will you describe the status of nutrition education in your medical curriculum? Probe further

In what ways have you learned about nutrition, if at all? Probe further

What is responsible for the current status of nutrition in your medical curriculum? Probe further

In what ways do you think your medical programme needs improvement in nutrition? Probe further

In what ways could nutrition be improved in your medical programme? Probe further

\section{Abbreviations}

PBL/COBES: Problem-based learning/Community-based Education and Service; UDSSMHS: University for Development Studies, School of Medicine and Health Sciences; NHRCIRB: Navrongo Health Research Centre Institutional Review Board; 


\section{Acknowledgement}

Authors wish to thank all students who agreed and consented to participate in the study. In addition, authors wish to acknowledge the efforts of Kwaku Appiah-Kubi of the Department of Applied Biology of the University for Development Studies for his assistance in editing the English language of the manuscript.

\section{Funding}

Not applicable

\section{Availability of data and material}

Data is available upon request from the corresponding author

\section{Authors' contribution}

VM conceived and designed the study; collected and performed data analysis and interpretation, and drafting of manuscript. FCJS, PAA, AA and AJJAS jointly undertook critical revision of the manuscript. All authors approved the manuscript for publication.

\section{Ethics approval and consent to participate}

Ethical approval for this study was granted by the Navrongo Health Research Centre Institutional Review Board (NHRCIRB) (Ethics Approval ID: NHRCIRB209), Ghana. Students gave a written consent prior to participating in the study. The data was handled in an anonymously and confidentially.

\section{Consent for publication}

Not applicable

\section{Competing interests}

The authors declare that they have no competing interests 


\section{Authors' details}

${ }^{1}$ Department of Health Professions Education and Innovative Learning, School of Medicine and Health Sciences, University for Development Studies, Ghana

${ }^{2}$ School of Health Professions Education, Faculty of Health, Medicine and Life Sciences, Maastricht University, The Netherlands

${ }^{3}$ Department of Community Nutrition, School of Allied Health Sciences, University for Development Studies, Ghana 


\section{References}

1. Gibbs J, Cull W, Henderson W, Daley J, Hur K, Khuri SF. Preoperative serum albumin level as a predictor of operative mortality and morbidity: results from the National VA Surgical Risk Study. Arch Surg 1999;134(1):36-42.

2. Halsted CH. Clinical nutrition education - relevance and role models. Am J Clin Nutr 1998;67(2):192-196.

3. Montgomery DL, Splett PL. Economic benefit of breast-feeding infants enrolled in WIC. J Am Diet Assoc 1997;97(4):379-385.

4. van Weel C. Morbidity in family medicine: the potential for individual nutritional counseling, an analysis from the Nijmegen Continuous Morbidity Registration. Am J Clin Nutr 1997;65(6 Suppl):1928S-1932S.

5. St Jeor ST, Krenkel JA, Plodkowski RA, Veach TL, Tolles RL, Kimmel JH. Medical nutrition: a comprehensive, school-wide curriculum review. Am J Clin Nutr 2006;83(4):963S-967S.

6. Eckel R, Jakicic J, Ard J, et al. AHA/ACC guideline on lifestyle management to reduce cardiovascular risk. A report of the ACC/AHA task force on Practiceguideline. Circulation 2014;2013:1524-4539.

7. Hoyert DL, Xu J. Deaths: preliminary data for 2011. Natl Vital Stat Rep. 2012;61(6):1-52.

8. Kris-Etherton PM, Akabas SR, Bales CW, et al. The need to advance nutrition education in the training of health care professionals and recommended research to evaluate implementation and effectiveness. Am J Clin Nutr 2014;99(5):1153S-1166S.

9. Wynn K, Trudeau JD, Taunton K, Gowans M, Scott I. Nutrition in primary care Current practices, attitudes, and barriers. Can Fam Physician 2010;56(3):e109-e116.

10. Tziraki C, Graubard BI, Manley M, Kosary C, Moler JE, Edwards BK. Effect of Training on Adoption of Cancer Prevention Nutrition-Related Activities by Primary Care Practices: Results of a Randomized, Controlled Study. J Gen Intern Med 2000;15(3):155-162.

11. Glanz K, Gilboy MB. Physicians, preventive care, and applied nutrition: selected literature. Acad Med 1992;67(11):776-781.

12. Krause T, Fox H. Nutritional knowledge and attitudes of physicians. J Am Diet Assoc 1977;70(6):607-609.

13. Soltesz KS, Price JH, Johnson LW, Telljohann SK. Perceptions and practices of family physicians regarding diet and cancer. Am J Prev Med 1994;11(3):197-204.

14. Crowley J, Ball L, Han DY, Arroll B, Leveritt M, Wall C. New Zealand Medical Students Have Positive Attitudes and Moderate Confidence in Providing Nutrition Care: A Cross-Sectional Survey. J Biomed Educ 2015:2015:7

15. Winick M. Nutrition education in medical schools. Am J Clin Nutr 1993;58(6):825-827.

16. Chisholm A, Mann K, Peters S, Hart J. Are medical educators following General Medical Council guidelines on obesity education: if not why not? BMC Med Educ 2013;13:53-53.

17. Mihalynuk TV, Coombs JB, Rosenfeld ME, Scott CS, Knopp RH. Survey correlations: Proficiency and adequacy of nutrition training of medical students. J Am Coll Nutr 2008;27(1):59-64.

18. Adams KM, Lindell KC, Kohlmeier M, Zeisel SH. Status of nutrition education in medical schools. Am J Clin Nutr 2006;83(4):941S-944S.

19. Adams KM, Kohlmeier M, Powell M, Zeisel SH. Nutrition in Medicine: Nutrition Education for Medical Students and Residents. Nutr Clin Pract 2010;25(5):471-480.

20. Chung M, van Buul V, Wilms E, Nellessen N, Brouns F. Nutrition education in European medical schools: results of an international survey. Euro J Clin Nutr 2014;68(7):844-846.

21. Gramlich LM, Olstad DL, Nasser R, et al. Medical students' perceptions of nutrition education in Canadian universities. Appl Physiol Nutr Metab 2010;35(3):336-343.

22. Schulman J. Nutrition education in medical schools: trends and implications for health educators. Med Educ Online 1999;4(1):4307

23. Walker WA. Innovative teaching strategies for training physicians in clinical nutrition: an overview. J Nutr 2003;133(2):541S-543S.

24. Touger-Decker R. Nutrition education of medical and dental students: innovation through curriculum integration. Am J Clin Nutr 2004;79(2):198-203. 
25. Intersociety Professional Nutrition Education Consortium. Bringing physician nutrition specialists into the mainstream: rationale for the Intersociety Professional Nutrition Education Consortium. Am J Clin Nutr 1998;68(4):894-898.

26. Levine BS, Wigren MM, Chapman DS, Kerner J, Bergman R, Rivlin R. A national survey of attitudes and practices of primary-care physicians relating to nutrition: strategies for enhancing the use of clinical nutrition in medical practice. Am J Clin Nutr 1993;57(2):115-119.

27. Ball LE, Hughes RM, Leveritt MD. Nutrition in general practice: role and workforce preparation expectations of medical educators. Austr J Prim Health 2010;16(4):304-310.

28. Wong V, Millen BE, Geller AC, Rogers AE, Maury J-J, Prout MN. What's in store for medical students? Awareness and utilization of expert nutrition guidelines among medical school preceptors. Prev Med 2004;39(4):753-759.

29. Mogre V, Scherpbier A, Dornan T, Stevens F, Aryee PA, Cherry MG. A realist review of educational interventions to improve the delivery of nutrition care by doctors and future doctors. Syst Rev 2014;3:148

30. Bandura A, McClelland DC. Social learning theory. Oxford: Prentice Hall 1977.

31. Crothers LM, Hughes TL, Morine KA. Theory and cases in school-based consultation: A resource for school psychologists, school counselors, special educators, and other mental health professionals: CRC Press: Boca Raton;2008.

32. Guba EG, Lincoln YS. Competing paradigms in qualitative research. Handbook of qualitative research. In: N. K. Denzin \& Y. S. Lincoln. Editors. Handbook of qualitative research. London: Sage; 1994. p. 105-17.

33. Crotty $M$. The foundations of social research: Meaning and perspective in the research process. London; Thousand Oaks, Sage Publications;1998.

34. McLellan L, Yardley S, Norris B, de Bruin A, Tully MP, Dornan T. Preparing to prescribe: How do clerkship students learn in the midst of complexity? Adv Health Sci Educ 2015;20(5):1339-1354.

35. Mills J, Bonner A, Francis K. The development of constructivist grounded theory Int J Qual Methods 2006;5(1):25-35

36. Watling $C$, Driessen $E$, Vleuten $C P$, Vanstone $M$, Lingard L. Music lessons: revealing medicine's learning culture through a comparison with that of music. Med educ 2013;47(8):842-850.

37. Watling CJ, Lingard L. Grounded theory in medical education research: AMEE Guide No. 70. Med teach 2012;34(10):850-861.

38. Gill P, Stewart K, Treasure E, Chadwick B. Methods of data collection in qualitative research: interviews and focus groups. Br Dent J 2008;204(6):291-295.

39. Britten N. Qualitative research: qualitative interviews in medical research. BMJ 1995;311(6999):251-253.

40. Martin L, de van der Schueren MA, Blauwhoff-Buskermolen S, Baracos V, Gramlich L. Identifying the Barriers and Enablers to Nutrition Care in Head and Neck and Esophageal Cancers An International Qualitative Study. J Parenter Enteral Nutr 2016;40(3):355-366.

41. Corbin J, Strauss A. Basics of qualitative research: Techniques and procedures for developing grounded theory. 2nd ed. London: Thousand Oaks, Sage Publications;2008..

42. Charmaz K. Constructing grounded theory: A practical guide through qualitative analysis. London: Thousand Oaks, Sage Publications; 2006.

43. Williams KN, Ramani S, Fraser B, Orlander JD. Improving bedside teaching: findings from a focus group study of learners. Acad Med 2008;83(3):257-264.

44. Crowley J, Ball L, Han DY, et al. Doctors' attitudes and confidence towards providing nutrition care in practice: Comparison of New Zealand medical students, general practice registrars and general practitioners. J Prim Health Care 2015;7(3):244-250.

45. Crowley J, Ball L, Han DY, Arroll B, Leveritt M, Wall C. New Zealand medical students have positive attitudes and moderate confidence in providing nutrition care: a cross-sectional survey. J Biomed Educ 2015;2015:7.

46. Spencer EH, Frank E, Elon LK, Hertzberg VS, Serdula MK, Galuska DA. Predictors of nutrition counseling behaviors and attitudes in US medical students. Am J Clin Nutr 2006;84(3):655-662.

47. Adams KM, Kohlmeier M, Zeisel SH. Nutrition Education in US Medical Schools: Latest Update of a National Survey. Acad Med 2010;85(9):1537-1542. 
48. Walker WA. Advances in nutrition education for medical students - Overview. Am J Clin Nutr 2000;72(3):865S-867S.

49. Weinsier RL, Boker JR, Feldman EB, Read MS, Brooks CM. Nutrition knowledge of senior medical students: a collaborative study of southeastern medical schools. Am J Clin Nutr 1986;43(6):959-968.

50. Hyska J, Mersini E, Mone I, et al. Assessment of knowledge, attitudes and practices about public health nutrition among students of the University of Medicine in Tirana, Albania. South East Euro J Public Health. 2015;1(1).

51. Lo C. Integrating nutrition as a theme throughout the medical school curriculum. Am J Clin Nutr 2000;72(3):882s-889s.

52. Taren DL, Thomson CA, Koff NA, et al. Effect of an integrated nutrition curriculum on medical education, student clinical performance, and student perception of medical-nutrition training. Am J Clin Nutr 2001;73(6):1107-1112.

53. Krebs NF, Primak LE. Comprehensive integration of nutrition into medical training. Am J Clin Nutr 2006;83(4):945S-950S.

54. Hafferty FW. Beyond curriculum reform: confronting medicine's hidden curriculum. Acad Med 1998;73(4):403-407.

55. Hughes L. Creating an interprofessional workforce: An education and training framework for health and social care in England. London: Dept. of Health/CAIPE. 2007.

56. Ball L, Crowley J, Laur C, Rajput-Ray M, Gillam S, Ray S. Nutrition in medical education: reflections from an initiative at the University of Cambridge. J Multidiscip Healthc 2014;7:209.

57. Bridges DR, Davidson RA, Odegard PS, Maki IV, Tomkowiak J. Interprofessional collaboration: three best practice models of interprofessional education. Med Educ Online. 2011;16.

58. Friedman G, Kushner R, Alger-Mayer S, Bistrian B, Gramlich L, Marik PE. Proposal for Medical School Nutrition Education Topics and Recommendations. J Parenter Enteral Nutr 2010;34(6 suppl):40S-46S.

59. Pearson TA, Stone EJ, Grundy SM, McBride PE, Van Horn L, Tobin BW. Translation of nutritional sciences into medical education: the Nutrition Academic Award Programme. Am J Clin Nutr 2001;74(2):164-170.

60. Hillenbrand KM, Larsen PG. Effect of an educational intervention about breastfeeding on the knowledge, confidence, and behaviors of pediatric resident physicians. Pediatrics 2002;110(5).

61. Mogre V, Scherpbier AJ, Stevens F, Aryee P, Cherry MG, Dornan T. Realist synthesis of educational interventions to improve nutrition care competencies and delivery by doctors and other healthcare professionals. BMJ Open 2016;6(10):e010084.

62. Buchowski MS, Plaisted C, Fort J, Zeisel SH. Computer-assisted teaching of nutritional anemias and diabetes to first-year medical students. Am J Clin Nutr 2002;75(1):154-161.

63. Maiburg BHJ, Rethans JE, Schuwirth LWT, Mathus-Vliegen LMH, van Ree JW. Controlled trial of effect of computer-based nutrition course on knowledge and practice of general practitioner trainees. Am J Clin Nutr 2003;77(4S):1019S-1024.

64. Carson JAS, Gillham MB, Kirk LM, Reddy ST, Battles JB. Enhancing self-efficacy and patient care with cardiovascular nutrition eduction. Am J Prev Med 2002;23(4):296-302. 


\section{Chapter}

PROTOCOL:

\section{A realist review of educational interventions to improve the delivery of nutrition care by doctors and future doctors}

Victor Mogre, Albert Scherpbier, Timothy Dornan, Fred Stevens, Paul Aryee and M. G. Cherry Systematic Reviews 2014; 3:148. DOI: 10.1186/2046-4053-3-148 


\section{Abstract}

Background: Dietary interventions are considered an important aspect of clinical practice, more so in the face of the rising prevalence of obesity, diabetes and cardiovascular diseases globally. Routinely, most doctors do not provide such intervention to their patients, and several barriers, present during both training and clinical practice, have been identified. Educational interventions to improve nutrition care competencies and delivery have been implemented, but with variable success, probably, due to the complex nature of such interventions. Using only traditional methods only, to investigate whether interventions are effective or not may not provide appropriate lessons. It is therefore pertinent to conduct a realist review that investigates how the interventions work. This realist review aims at determining what sort of educational interventions work, how, for whom, and in what circumstances, to improve the delivery of nutrition care by doctors and future doctors.

Methods/design: This realist review will be conducted according to Pawson's five practical steps for conducting realist review: (1) clarifying the scope of the review, (2) determining the search strategy, including adopting broad inclusion/exclusion criteria and purposive snowballing techniques, (3) ensuring proper article selection and study quality assessment using multiple methods, (4) extracting and organising data through the process of note taking, annotation and conceptualization, and (5) synthesising the evidence and drawing conclusions through a process of reasoning. This realist review protocol has not been registered in any data base before now.

Discussion: Findings will be reported according to the publication criteria outlined by the realist and meta-narrative evidence synthesis (RAMESES) group.

Key words: Realist review, Realist synthesis, Educational interventions, Nutrition care, Future doctors, Improve, Delivery 


\section{Background}

Dietary interventions, such as discussing weight loss and providing dietary counselling to obese patients, are an important part of clinical practice. Most doctors, however, do not routinely provide them ${ }^{1,2}$. There are several reasons for this, including lack of time and lack of confidence that patients will comply ${ }^{1}$. In addition, doctors lack confidence, motivation, skills and knowledge, which results from inadequate training during medical school 1,3-5. Fifty percent of graduating US medical students report that the time devoted to nutrition education in medical school and the nutritional content of their curricula are inadequate ${ }^{6}$.

On the other hand, doctors have generally positive views on the role of nutrition in clinical practice ${ }^{1,2}$ and would give nutrition care (for example dietary counselling) to their patients if it were not for the various barriers outlined above ${ }^{1}$. Those barriers are present during both training and practice. Educational interventions to improve medical students' and doctors' nutrition care competencies and delivery have been undertaken during training and in practice with variable success ${ }^{7-9}$. The delivery of nutrition care by doctors is therefore still in need of appropriate models ${ }^{7-9}$. It is important to identify key components of effective educational interventions and which educational interventions and strategies should be used in which settings ${ }^{10}$.

Reviews on the effectiveness of educational interventions to improve the nutrition care competencies and delivery of doctors are limited. In our literature search we came across only one review that considered the effectiveness of nutrition training programmes to improve health workers' (including doctors) nutrition knowledge and competence to manage child under nutrition ${ }^{11}$. The review by Sunguya et al., followed a traditional systematic review process. To the best of our knowledge, no review be it traditional or realist, has considered the effectiveness of educational interventions to improve the nutrition care competencies of future doctors only and/or both doctors and future doctors. This is probably due to the complex nature of such interventions. As such there is paucity of data exploring how educational interventions might work most effectively, how, why (the mechanisms beneath) and under what circumstances to improve the delivery of nutrition care by future doctors and doctors. This review aims to fill these gaps.

The strength of systematic reviews is that they can measure the effectiveness of interventions in terms of their effect sizes. They aspire to be relatively free of context effects, which confound the relationship between an intervention and its outcome. In the words of Cook et al., ${ }^{12}$, classical systematic reviews are "justification research". Systematic review was introduced in the biomedical domain to help doctors choose between different treatments ${ }^{13,14}$. There is a preference within the education domain, however, for "clarification research", which seeks not so much to find out whether an intervention works but as to how and why it works ${ }^{12}$. From that perspective, systematic review methodology has shortcomings: notably, that the drive to minimise bias over-simplifies the inherent complexity of interventions and their inherently contextual nature ${ }^{15,16}$. This may 
result in misleading oversimplification and an inability to account for the inescapable complexity of interactions between conditions, mechanisms, and outcomes ${ }^{17}$.

In this realist review, we conceptualise educational interventions and strategies to improve the delivery of nutrition care as complex ones ${ }^{18}$, which involve multiple actors (teachers, learners, patients, health care providers, etc.) operating at different levels, the artefacts they use, and the material environments in which they work ${ }^{17}$. In a non-linear fashion, these components interact to produce context-dependent outcomes.

The broad purpose of this realist review is to determine what sort of educational interventions work, how, for whom, why and in what circumstances to improve the delivery of nutrition care by future doctors and doctors. Considering educational interventions to improve the delivery of nutrition care by future doctors as well as those to improve the delivery of nutrition of doctors, will grant us the opportunity to identify mechanisms and contextual factors that influences those educational interventions during training and in practice. This will provide curriculum leaders and policy makers with evidence, which will help them, design and implement educational interventions that are appropriate to the settings, people, and educational goals they are aiming to achieve. Specifically, it sets out to answer the following review questions:

\section{Review questions}

1. What learning outcomes do future doctors and doctors need to attain in order to deliver nutrition care to patients?

2. What mechanisms and contextual factors lead to the attainment of those outcomes?

3. How do those mechanisms and contextual factors interplay to produce those outcomes??

4. How could undergraduate educational interventions support an optimal interplay of those factors?

\section{Methods/design}

\section{Study design}

As informed by the above research questions, the realist review will follow principles outlined by Pawson ${ }^{16}$. These principles emphasize the search for evidence supporting complex interventions and providing explanations for why they may or may not work, how, and in what contexts ${ }^{16,19}$. Fundamentally, realist review is concerned with developing and refining theory ${ }^{16,20,21}$, accounting for context as well as outcomes in the process of systematically, iteratively, and transparently synthesizing relevant literature ${ }^{22,23}$. It aims to unpack a context - mechanism- outcome relationship (CMO), explaining examples of success, failure and various eventualities in between ${ }^{24}$. The theoretical explanations that result from this process are further configured and refined to construct an 'overarching' 
realist programme theory ${ }^{25}$. The key product of this review, therefore is the building and refinement of a programme theory which enables us to achieve a level of abstraction needed to understand the diversity of outcomes produced in different contexts ${ }^{26}$. The programme theory of an intervention is the specification of how and why the programme or intervention is conceived to cause its intended outcomes, taking into consideration the active mechanisms and contexts ${ }^{26}$. The theory is built based on available evidence and refined throughout the study.

The review will be conducted following Pawson's five practical steps for conducting a realist review ${ }^{20}$. These steps include: 1 ) clarifying the scope of the review; (2) determining the search strategy; (3) ensuring proper article selection and study quality assessment; (4) extracting and organising data and (5) synthesising the evidence and drawing conclusions through a process of reasoning ${ }^{16,20}$.

\section{Theory initiation}

An important component and obviously the end goal of the realist review process is the development or refinement of a programme theory (ies). As suggested by Pawson ${ }^{16,20}$, we will tap into stakeholders and experts to help in this regard. Exploration of key theories has already begun through on-going conversations within the review team and discussions with health professions educational experts, teachers, students, physicians, among others. A concept map is being developed of ideas, views and opinions on why educational interventions work, who they work for, in what circumstances and why. This will be explored in the literature searches "to identify the theories, hunches, expectations, and the rationalizations" ${ }^{16}$ for why educational interventions may or may not work to improve the delivery of nutrition care ${ }^{16,20}$ by future doctors and doctors. This exercise is aimed at identifying "a range of theories and explanations for how educational interventions are supposed to work (and for whom), when they do work, when they don't achieve the desired change in nutrition care delivery, why they are not effective in this and why they are not being used"27. These initial on-going processes have resulted in the following candidate programme theories which will be continuously refined and strengthened as they transform (or emerging theories arises) and finalized through the testing of their validity during the realist review process.

\section{Candidate programme theories}

Riding on the back of existing substantive theories of expertise development ${ }^{28-31}$, the delivery of nutrition care is a complex skill that is influenced by: (1) nutrition care competency, (2) the individual motivation of the future doctor/doctor, self-regulation and metacognition and (3) the workplace setting (e.g. the hospital). The delivery of nutrition care by doctors is a capability that is influenced by cognitive and affective processes (knowledge, skills, attitudes, self-efficacy, etc.) of the individual as well as the social, cultural and physical environmental context in which the behaviour is executed. 
Nutrition care competency is a broad concept that encompasses knowledge, skills, and attitudes. It is the capability to apply or use nutrition knowledge and skills/abilities needed to successfully perform the task of delivering nutrition care to patients in a defined work setting (e.g. the hospital). We recognise that the delivery of nutrition care is influenced by the availability of adequate competency which is the outcome of a learning process that is influenced by factors within the academic environment relating to the quantity, quality and nutrition content in the curriculum as well as the teaching and learning methods employed and the reinforcement experienced by the individual and by others. It is our hypothesis that doctors will deliver nutrition care by a process of mimesis ${ }^{32}$, which means building on and making personal behaviours and attributes demonstrated by colleagues (e.g. course mates) and superiors (e.g. specialist, consultants, clinical teachers, etc.) delivering nutrition care.

The delivery of nutrition care is also influenced by the doctor's own thoughts, selfbeliefs and their interpretation of the environment relating to Bandura's social cognitive theory ${ }^{33}$. Doctors are more likely to deliver nutrition care to their patients if they have high self-efficacy for it and less likely to deliver nutrition care if they do not feel self-efficacious. In accordance with the social cognitive theory, knowledge (cognitive), behavioural, personal, and environmental factors interact to determine motivation and behaviour $^{34}$.

Even though nutrition care competency is an important determinant of the delivery of nutrition care, it does not alone predict the delivery of nutrition care for the promotion of health and well-being ${ }^{3}$. The delivery of nutrition care is also influenced by the social environment (the workplace setting). We assume that the delivery of nutrition care by doctors is a behaviour demonstrated in the social context of the workplace, which is influenced by observing and modelling the behaviours, attitudes, and emotional reactions of others (e.g. superiors) ${ }^{35}$. It is also influenced by the structural determinants of behaviours such as the nature of the workplace setting (e.g. hospital/community, emergency/paediatric/general ward), job descriptions/role, time and availability of other staff to undertake particular roles.

The interaction of these three factors (nutrition care competencies, the individual's motivation and workplace setting) can be likened to Wood and Bandura's ${ }^{36}$ Triadic Reciprocal Determinism model, in that the on-going functioning of nutrition care delivery is the product of a continuous interaction between cognitive, behavioural, and social-environmental factors. Therefore our candidate programme theory is that the delivery of nutrition care is a complex process that is not only influenced by cognitive skills, concepts and abstract rules but the individual's motivation as well as the social environment within which nutrition care is delivered (see Figure 1). 


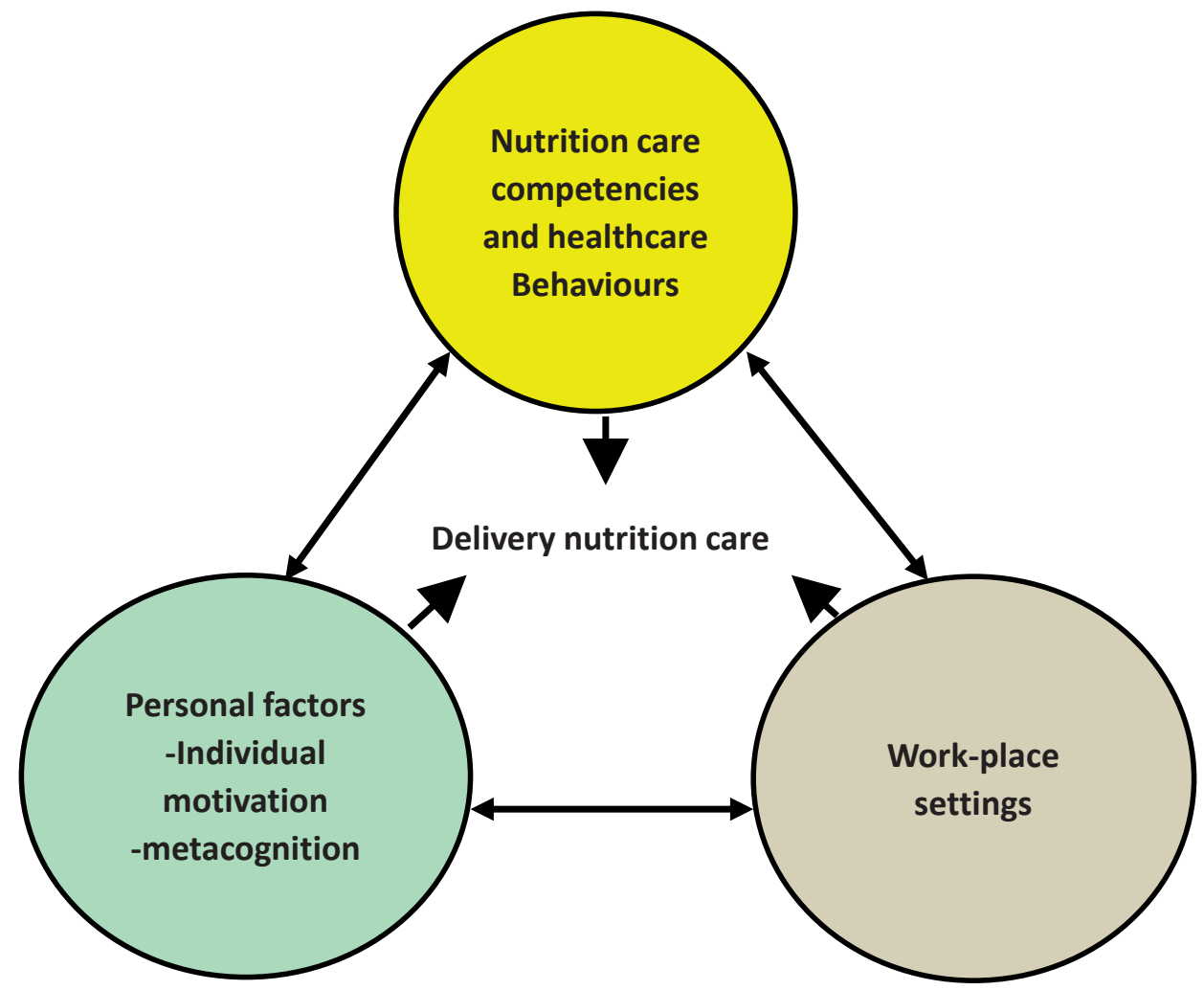

Figure 1: Theoretical model (Adapted from Wood and Bandura's Triadic Reciprocal Determinism ${ }^{36}$ )

\section{Determining the search strategy}

As is customary in realist review, broad inclusion/exclusion criteria will be employed to ensure that the process of programme theory development takes in the widest range of evidence $^{20}$.

\section{Inclusion criteria}

Types of data

In keeping with the nature of realist review, both quantitative and qualitative studies will be included. Both published and grey literature (such as websites, reports of international organizations as well as dissertations and theses) will be consulted.

\section{Study design}

Randomised control trials (RCTs), cluster RCTs, quasi-experimental studies, pre-post-intervention longitudinal studies (with or without comparison groups), non-interventional qualitative studies, cross-sectional studies and case reports will be included. 


\section{Participants}

Studies including healthcare professions students (including medicine) astearners will be included. In addition, those having doctors as learners will also be included.

\section{Search limitations}

The search will be limited to a 20-year-publication period (1994-2014), in order to restrict the research to relatively recent evidence whilst ensuring sufficient evidence.

\section{Study focus}

Studies with nutrition training/educational interventions will be included. Non-interventional studies will also be considered if they provide information about how conditions, mechanisms, and outcomes interrelate.

\section{Outcome measures}

All studies that have nutrition competencies and behaviours and/or delivery of nutrition care as the outcome measure will be considered

\section{Exclusion criteria}

Studies will be excluded if they are not relevant to nutrition care competencies and/or delivery, or if they are purely anecdotal.

\section{Search process}

A combination of $\mathrm{MeSH} /$ thesauri and free text terms will be employed in the search strategy. Truncation and appropriate Boolean operators will be employed. Scoping searches will be conducted to refine the search terms further. The terms will be broad and will be grouped into participants, outcomes and conditions as shown below

- Participants. Medical students; health professions students; general practitioners; doctors; primary care physicians

- Outcomes. Diet counselling; nutrition education; eating behaviours; preventive medicine; nutritional management; lifestyle modification; nutrition therapy

- Conditions. Curriculum; integrated; training; evaluation; assessment; impact; learning; undergraduate; medical education; capacity; capacity building

Searches will be conducted across the following online databases: MEDLINE, CINAHL, ERIC, Embase, PsycINFO, Sociological Abstracts, Web of Science and Google Scholar and any other database related to medical education. Grey literature and thesis databases will also be consulted. Recognizing the limitations of a traditional search strategy ${ }^{37}$, an expert-directed search using a multiple-search strategy approach will be incorporated. As stated by Kastner (2011), a multiple-search strategy approach seeks to explore and contextualize the intervention in multiple settings resulting in the strategy being iterative 
and interactive ${ }^{15,16}$. This process is flexible and allows us the benefit of capitalising on unanticipated findings ${ }^{27}$. Experts will be identified through snowball searching. These experts will then be consulted to help us look for and find potentially relevant literature and concepts. The reference list of relevant articles from the core articles will be used to find potentially relevant studies from bibliographic references.

As expected in interpretive research like this realist review, the search for new studies will end at the point of theoretical saturation, a point at which the evidence available meets the theoretical need or answers the research questions ${ }^{16,20,38}$. In consonance with the suggestion of Pawson ${ }^{16,20}$ the test of saturation will be obtained iteratively. At each stage or cycle the latest addition of literature would be assessed to determine whether something new has been added to our understanding of the programme theory.

A reviewer will ensure that all relevant journals and studies are adequately indexed in the databases selected. In addition, members of the research team will review the final list of studies to identify and correct obvious omissions using their knowledge. Potentially eligible studies based on the inclusion and exclusion criteria described above will be obtained in full text and downloaded into Reference Manager Professional Version 11.0. The content of the file will be backed-up to a secured server. Shown in Figure 2 is a diagram of the proposed search strategy. 


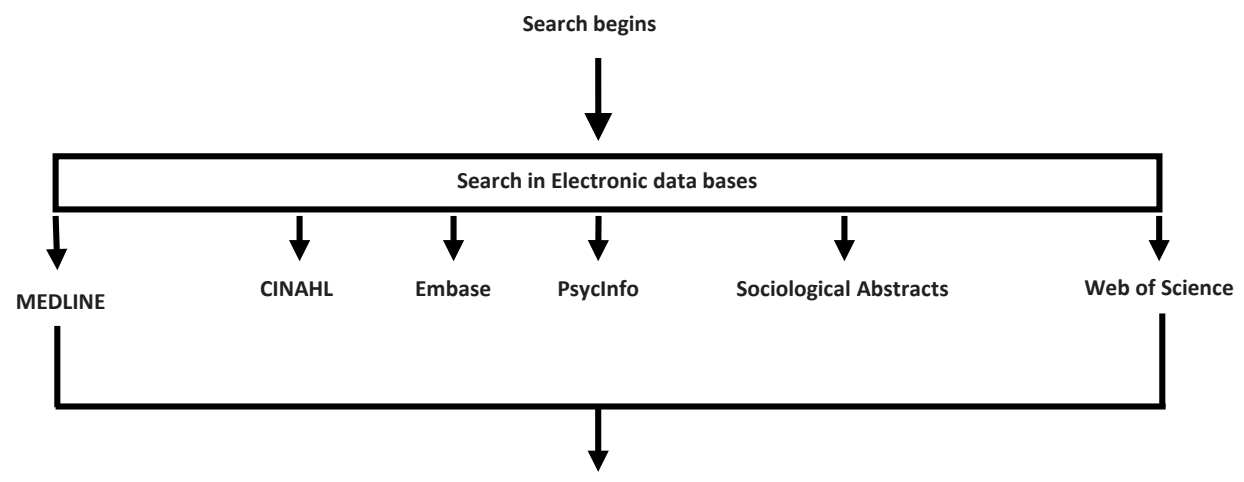

Initial results to be imported into reference manager

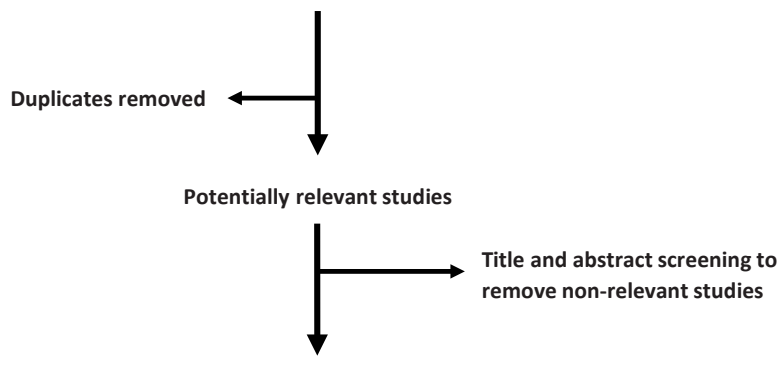

Full text retrieval of relevant articles for data extraction

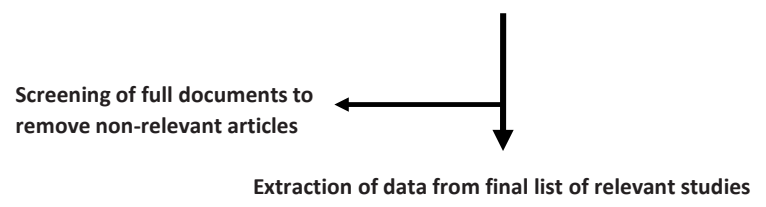

Figure 2: Search strategy

\section{Selection of studies and quality assessment}

Unlike systematic reviews, the quality assessment of studies will not be based on the hierarchy of evidence ${ }^{20}$. Pawson argues that multiple methods of assessment should be employed as a measure of quality in realist synthesis in order to "illuminate the richest picture" 20,27. The heuristic approach which will be employed is one where the value of a study is assessed by how much it is able to enrich the programme theory. It should answer the question: 'Does the research address the theory under investigation?' Usually, the unit of assessment is not the study itself but sections of related theory and evidence ${ }^{16}$.

As an initial step, two research team members in consultation with the wider group will independently screen the title, abstract, and subject headings of the searched studies against the initial inclusion and exclusion criteria (which will evolve during the process of the review). This initial process by the two reviewers will afford us the opportunity to 
maintain a certain level of rigour (i.e. refinement and proper interpretation of the inclusion and exclusion criteria). It is also a valuable platform for reflexive discussion that will enable informed decisions among reviewers for identifying relevant data ${ }^{39}$. As we anticipate a high volume of potentially relevant studies, provided there is high level of agreement between the two reviewers, this initial process may reduce the number of studies that will need to be reviewed further.

A key step of our review is the process of determining rigour. Pawson describes the process of determining "rigour" "as whether a particular inference drawn by the authors has sufficient evidence to make a methodologically credible contribution to the test of a theory" and to apply "judgment" to supplement formal critical appraisal checklists (if they are used ${ }^{20}$. In the absence of technical procedures to describe "judgements" we would adopt an approach that will use rigour (e.g. methodological rigour) as a supporting/complementary/mediating tool rather than the only means to determine the quality of a study. This will grant us the opportunity to include studies that best inform our purpose (e.g. for studies that have the same concepts but with differing methodological rigour or to adjudicate between studies that have similar methodologies but conflicting results). Judgement will be used to resolve conflicts or disagreements between reviewers by determining how the results of the study (ies) could be applied to the context of using educational interventions to improve the delivery of nutrition care by future doctors and doctors. This will be done through discussions among members of the review team. In order not to exclude studies based on methodological rigour, studies will be selected and reviewed based on what new knowledge (which could come from any part of the study including introduction, methodology, results and discussion sections) it brings on board for the development and refinement of the programme theory. Furthermore the meaning and value of rigour will be defined, examined and documented for each selected study to ensure transparency. This will include decisions about rigour) and relevance (the contribution the study either as a whole or a section makes to the review). If need be and appropriate, appraisal tools may also be used to determine rigour. Similar to systematic reviews the importance of transparency in realist review is to ensure the validity, reliability and verifiability of findings and conclusions ${ }^{27,40}$.

\section{Data extraction and organisation}

In meeting the requirements of this theory-driven approach, our review will include all potentially relevant studies that add to the development of our theory ${ }^{41}$. Data "extraction" and organisation will be done through a process of note taking, annotation and conceptualization. In addition, our conceptualization of the pertinent processes that constitute the implementation of educational interventions to improve the delivery of nutrition care by future doctors and doctors will be refined.

In order to build and refine our programme theory/theories, a set of basic information from the selected studies will be collected into a standard data extraction form by the 
first author. To ensure accuracy, the initial results of this process will be cross-checked by another reviewer; emphasis on the data extraction fields involving qualitative data or key information (e.g. study design). Disagreements will be resolved by involving other members of the review team. Each study will be read and re-read to capture themes and concepts that might contribute to the building and refinement of the theory. The entire data extraction process will involve critical discussion between the two reviewers (first author and the cross-checking reviewer) and the wider team to ensure that data are used to develop a line of argument(s) that feeds into the final synthesis stage rather than simply classifying them.

\section{Data analysis and synthesis}

In a realist reviews, data analysis and synthesis are iterative processes that may occur sequential or in parallel ${ }^{19}$. Data analysis in a realist review aims to analyse data using realist concepts employing a philosophical "eye" ${ }^{19}$. Realism is based on a generative explanation for causation; outcomes (O) are generated by relevant mechanisms (M) activated in a context $(\mathrm{C})$ resulting in a $\mathrm{CMO}$ configuration. The key task is to refine programme theory that in general explains what works, for whom, in what circumstances, in what respects and why ${ }^{42}$. Pawson et al. suggest that synthesis partly consists of processes of reasoning including juxtaposing, reconciling, adjudicating, consolidating and situating all sources of evidence that helps to refine the programme theory ${ }^{15,16}$. We believe that these processes are insufficient on their own as they do not provide a complete description of synthesis of the data. To cater for these limitations, we include the principles of thematic analysis to analyse and synthesize our data. It is an appropriate compromise that combines the principles of meta-ethnography and grounded theory ${ }^{43}$. Reciprocal "translation" a feature of meta-ethnography is realised through the coding of findings to develop descriptive and analytical themes. As a feature of grounded theory adapted into thematic analysis, the process is inductive and the development of themes is through the process of constant comparison ${ }^{43}$. Recognising the basic principles of realist synthesis and thematic analysis we intend to analyse and synthesize our data according to the following steps, a modification of the approach proposed by Rycroft-Malone et al ${ }^{42}$ :

a. Extracted data will be summarised and organised into theory areas and review questions related tables.

b. Individual reviewers will independently theme the extracted data using "free codes"

c. Identified themes will be collated and discussed by members of the review team. This will provide an opportunity to discuss and resolve differences that may arise.

d. Through discussions among members of the review team, chains of inferences will be identified. A chain of inference refers to linkages between the various themes that have been identified ${ }^{42}$ as well as linking them to the primary data that generated the themes. This will be done by keeping track of the primary data that generated the themes. Proceeding, connections will be made among chains of inferences. This will 
be an iterative process in which chains of inferences will be linked to each other as well as to the primary source of evidence.

e. Hypotheses will be developed based on the chains of inferences ${ }^{42}$. Conceptually, themes will be linked to chains of inference, which will be linked to a hypothesis. All included studies will be linked explicitly to chains of inferences and hypotheses. The output of the previous steps will result in a cumulative picture of potential mechanisms, contexts and outcomes chains ${ }^{42}$. The generated hypotheses will then be used to either form new programme theories or refine them. Narrative synthesis will be developed around each programme theory (ies), summarizing the nature of the context, mechanism and outcome links and the characteristics of the primary evidence underpinning them (individual included studies) ${ }^{43}$. These will be presented using text, summary tables, a logic model and where appropriate graphics ${ }^{44}$.

The origins of the inferences, the hypothesis and the subsequent programme theories and their specific sources, on which they are grounded, will be documented and justified. The reasoning processes explaining how they are rooted in the primary evidence will be documented to ensure transparency. For example, how an included study contributed to a theme, which resulted in the formation of a chain of inference, subsequently resulting in a hypothesis and finally contributing to a programme theory. We will also document the inferential shifts that occur during the reasoning process to engage the evidence.

This realist review protocol has not been registered in any database.

\section{Discussion}

\section{Formation of a review advisory group}

In order to meet the requirement of involving stakeholders in the realist review, a review advisory group will be formed. The review advisory group will comprised of all members of this research team and will also include teachers, students, curriculum experts, doctors, among others. The composition of the group will depend on the stage of the realist review. The advisory review group will assist in building programme theories. The advisory review group will provide a "reality check" on the clarity and explanatory strength of identified and selected theories.

\section{Importance of the review}

The findings of the review may be useful to teachers, students, researchers in medical education as well as policy and decision makers in health professions education and to patients in the long-term. As indicated in the introduction section of this review protocol, the delivery of nutrition care by doctors is still an area in need of an appropriate model. This review will help in the realization of such models by identifying the context and 
mechanisms under which such models operate successfully. Also this review forms part of a PhD project aiming at developing an intervention to improve the nutrition care competencies and delivery of health care providers. This initial review will help explain the intrinsic reasons for why and under what conditions educational interventions improve nutrition care competencies during training and delivery in practice. This is an important first step toward a better understanding of characteristics of educational interventions to help improve nutrition care competencies and delivery.

A realist review of literature is considered a relatively new methodology and few researchers in health professions education have used it. Obviously, conceptual and methodological challenges might have contributed to this situation ${ }^{38}$. A secondary importance of this realist review is to facilitate its adoption by other researchers in health professions education. This will be realised through the publication of a methods paper through this review protocol and the findings of the review.

\section{Reporting and dissemination of findings}

Our findings will be reported according to the publication criteria outlined by the realist and meta-narrative evidence synthesis (RAMESES) group ${ }^{19}$. The research questions will be explored, answered and reported in a language that is acceptable to all stakeholders. The findings of the review will be reported through two activities. Firstly, we will report the findings in an international journal specializing in medical education, implementation science or public health or medical journals. Secondly, the results of the review will be presented in international conferences such as The NETWORK-Towards Unity for Health annual conference, AMEE and among others.

\section{Abbreviations}

RAMESES: Realist and Meta-narrative Evidence Synthesis. The NETWORK: TUFH: The Network Towards Unity for Health. AMEE: The Association for Medical Education in Europe

\section{Competing interest}

The authors declare that they have no competing interest. 


\section{Authors' contributions}

VM performed the conceptual work and drafted the protocol. AS, TD, FS and PA supervised the drafting of the protocol, critically, reviewed the text and assisted with editing. MGC provided important input regarding the methodology and revised the protocol with regard to the realist approach. All authors read and approved the final protocol

\section{Acknowledgements}

The authors wish to thank the Librarian of the School of Medicine and Health Sciences, University for Development Studies for his support in identifying the key words for the search process.

\section{Funding}

This protocol was self-funded as part of VM's PhD in Health Professions Education.

\section{Author details}

${ }^{1}$ Department of Human Biology, School of Medicine and Health Sciences, University for Development Studies, Tamale, Ghana. ${ }^{2}$ School of Health Professions Education, Maastricht University, Maastricht, The Netherlands. ${ }^{3}$ Department of Health Services Research, Institute of Psychology, Health and Society, University of Liverpool, Liverpool, UK. 


\section{References}

1. Kushner RF. Barriers to providing nutrition counseling by physicians: a survey of primary care practitioners. Prev Med 1995;24(6):546-552.

2. Galuska DA, Will JC, Serdula MK, Ford ES. Are health care professionals advising obese patients to lose weight? JAMA 1999;282(16):1576-1578.

3. Mihalynuk TV, Coombs JB, Rosenfeld ME, Scott CS, Knopp RH. Survey correlations: proficiency and adequacy of nutrition training of medical students. J Am Coll Nutr 2008;27(1):59-64.

4. Schucker B, Wittes JT, Cutler JA, et al. Change in physician perspective on cholesterol and heart disease: results from two national surveys. JAMA 1987;258(24):3521-3526.

5. Wechsler H, Levine S, Idelson RK, Rohman M, Taylor JO. The physician's role in health promotion: a survey of primary-care practitioners. New Engl J Med 1983.

6. Mihalynuk TV, Scott CS, Coombs JB. Self-reported nutrition proficiency is positively correlated with the perceived quality of nutrition training of family physicians in Washington State. Am J Clin Nutr 2003; 77(5):1330-1336.

7. McLaren DS. Nutrition in medical schools: a case of mistaken identity. Am J Clin Nutr 1994;59(5):960-963.

8. Weinsier RL. National Dairy Council Award for Excellence in Medical/Dental Nutrition Education Lecture, 1995: medical-nutrition education--factors important for developing a successful programme. Am J Clin Nutr 1995;62(4):837-840.

9. Gershoff SN. National Dairy Council Award for Excellence in Medical/Dental Nutrition Education Lecture, 1996: nutrition education--success or failure? Am J Clin Nutr. 1996;64(5):809-812.

10. Bhattacharyya O, Reeves S, Zwarenstein M. What is implementation research? Rationale, concepts, and practices. Res Soc Work Pract 2009;19(5):491-502.

11. Sunguya BF, Poudel KC, Mlunde LB, Urassa DP, Yasuoka J, Jimba M. Nutrition training improves health workers' nutrition knowledge and competence to manage child undernutrition: a systematic review. Front Public Health 2013;1:37

12. Cook DA, Bordage G, Schmidt HG. Description, justification and clarification: a framework for classifying the purposes of research in medical education. Med Educ 2008;42(2):128-133.

13. Cochrane AL. Effectiveness and efficiency: random reflections on health services. London: Nuffield Provincial Hospitals Trust. 1972:p85

14. Muir Gray J. Evidence-based healthcare: how to make health policy and management decisions. London: Churchill Livingstone. 1997:p53.

15. Pawson R, Greenhalgh T, Harvey G, Walshe K. Realist synthesis: an introduction. ESRC Research Methods Programmeme. Manchester: University of Manchester. 2004.

16. Pawson R. Evidence-based policy: A realist perspective: Sage; 2006.

17. Wong G, Greenhalgh T, Pawson R. Internet-based medical education: a realist review of what works, for whom and in what circumstances. BMC Med Educ 2010;10(1):12.

18. Craig P, Dieppe P, Macintyre S, Michie S, Nazareth I, Petticrew M. Developing and evaluating complex interventions: the new Medical Research Council guidance. BMJ 2008;337:a1655

19. Greenhalgh T, Wong G, Westhorp G, Pawson R. Protocol-realist and meta-narrative evidence synthesis: evolving standards (RAMESES). BMC Med Res Methodol 2011;11(1):115.

20. Pawson R, Greenhalgh T, Harvey G, Walshe K. Realist review-a new method of systematic review designed for complex policy interventions. J Health Serv Res Policy 2005;10(suppl 1):21-34.

21. Rycroft-Malone J, McCormack B, DeCorby K, Hutchinson A. Realist Synthesis: Method and Examples. The research process in nursing. In: Gerrish KLA. Research Process in Nursing. 3rd ed. Oxford, U.K.: Wiley Blackwell; 2010.

22. Avis M, Freshwater D. Evidence for practice, epistemology, and critical reflection. Nurs Philos 2006;7(4):216-224. 
23. Kelly M, Morgan A, Ellis S, Younger T, Huntley J, Swann C. Evidence based public health: a review of the experience of the National Institute of Health and Clinical Excellence (NICE) of developing public health guidance in England. Soc Sci Med 2010;71(6):1056-1062.

24. Wong G, Greenhalgh T, Westhorp G, Buckingham J, Pawson R. RAMESES publication standards: realist syntheses. BMC Med 2013;11(1):21.

25. Merton RK. On Sociological Theories of the Middle Range (1949). Ders., Social Theory and Social Structure, New York. 1968.

26. Pawson R. Middle-range realism. Arch Euro J Sociol 2000;41(2):283-325.

27. Kastner M, Estey E, Perrier L, et al. Understanding the relationship between the perceived characteristics of clinical practice guidelines and their uptake: protocol for a realist review. Implement Sci 2011;6:69.

28. Scardamalia M, Bereiter C. Surpassing Ourselves: An inquiry into the nature and implications of expertise: Open Court, Chicago; 1993.

29. Bereiter C, Scardamalia M. Educational relevance of the study of expertise. Interchange 1986;17(2):10-19.

30. Carol-anne EM, Regehr G, Mylopoulos M, MacRae HM. Slowing down when you should: a new model of expert judgment. Acad Med 2007;82(10):S109-S116.

31. McLellan L, Tully MP, Dornan T. How could undergraduate education prepare new graduates to be safer prescribers? Br J Clin Pharmacol 2012;74(4):605-613.

32. Billett S. Mimetic learning at work: Learning through and across professional working lives. In: Billett $S$, Harteis, C \& Gruber, H. . International Handbook of Research in Professional and Practice-based Learning. Dordrecht, The Netherlands: Springer; 2014.

33. van der Bijl JJ, Shortridge-Baggett LM. The theory and measurement of the self-efficacy construct. Res Theory Nurs Pract 2001;15(3):189-207.

34. Crothers LM, Hughes TL, Morine KA. Theory and cases in school-based consultation: A resource for school psychologists, school counselors, special educators, and other mental health professionals: CRC Press; 2008.

35. Bandura A, McClelland DC. Social learning theory. Oxford: Prentice Hall;1977.

36. Wood R, Bandura A. Social cognitive theory of organizational management. Acad Manag Rev 1989;14(3):361-384.

37. Greenhalgh T, Peacock R. Effectiveness and efficiency of search methods in systematic reviews of complex evidence: audit of primary sources. BMJ 2005;331(7524):1064.

38. Robert E, Ridde V, Marchal B, Fournier P. Protocol: a realist review of user fee exemption policies for health services in Africa. BMJ Open. 2012;2(1): e000706.

39. Greenhalgh T, Potts HW, Wong G, Bark P, Swinglehurst D. Tensions and Paradoxes in Electronic Patient Record Research: A Systematic Literature Review Using the Meta-narrative Method. Milbank Q 2009;87(4):729-788.

40. Greenhalgh T, Robert G, Bate P, Macfarlane F, Kyriakidou O. Diffusion of innovations in health service organisations: a systematic literature review: John Wiley \& Sons; 2008.

41. Pearson $\mathrm{M}$, Chilton $\mathrm{R}$, Woods $\mathrm{HB}$, et al. Implementing health promotion in schools: protocol for a realist systematic review of research and experience in the United Kingdom (UK). Syst Rev 2012;1(1):48.

42. Rycroft-Malone J, McCormack B, Hutchinson AM, et al. Realist synthesis: illustrating the method for implementation research. Implement Sci2012;7(1):33.

43. Barnett-Page E, Thomas J. Methods for the synthesis of qualitative research: a critical review. BMC Med Res Methodol2009;9(1):59.

44. Brennan N, Bryce M, Pearson M, Wong G, Cooper C, Archer J. Understanding how appraisal of doctors produces its effects: a realist review protocol. BMJ Open 2014;4(6):e005466. 



\section{Chapter}

Realist synthesis of educational interventions to improve nutrition care competencies and delivery by doctors and other healthcare professionals 


\section{ABSTRACT}

Objective: To determine what, how, for whom, why, and in what circumstances educational interventions to improve the delivery of nutrition care by doctors and other healthcare professionals work?

Design: Realist synthesis following a published protocol and reported following Realist and Meta-narrative Evidence Synthesis: Evolving Standards (RAMESES) guidelines. A multidisciplinary team searched Medline, CINAHL, ERIC, EMBASE, PsyncINFO, Sociological Abstracts, Web of Science, Google Scholar, and Science Direct for published and unpublished (grey) literature. The team identified studies with varied designs; appraised their ability to answer the review question; identified relationships between contexts, mechanisms, and outcomes (CMOs); and entered them into a spreadsheet configured for the purpose. The final synthesis identified commonalities across $\mathrm{CMO}$ configurations.

Results: Over half of the 46 studies from which we extracted data originated from the US. Interventions that improved the delivery of nutrition care improved skills and attitudes rather than just knowledge; provided opportunities for superiors to model nutrition care; removed barriers to nutrition care in health systems; provided participants with local, practically relevant tools and messages; and incorporated non-traditional, innovative teaching strategies. Operating in contexts where student and qualified healthcare professionals provided nutrition care in both developed and developing countries, these interventions yielded health outcomes by triggering a range of mechanisms, which included: feeling competent; feeling confident and comfortable; having greater self-efficacy; being less inhibited by barriers in healthcare systems; and feeling that nutrition care was accepted and recognised.

Conclusion: These findings show how important it is to move education for nutrition care beyond the simple acquisition of knowledge. They show how educational interventions embedded within systems of healthcare can improve patients' health by helping health students and professionals to appreciate the importance of delivering nutrition care and feel competent to deliver it.

\section{STRENGTHS AND LIMITATIONS OF THIS STUDY}

1. Application of the principles of realist synthesis to nutrition and education research is novel.

2. The characteristics and conditions of educational interventions that can improve the delivery of nutrition care, identified by this review, are important to the work of policy makers, researchers, health professions educators, and course developers.

3. Few reports of failed educational interventions were found, indicating a risk of positive publication bias. 
4. Until our conceptual model is tested and refined in the real world, we consider it to be an indefinite candidate theory, presenting elements worth considering by those concerned with the design, implementation and evaluation of educational interventions to improve the delivery of nutrition care by doctors and other healthcare professionals.

5. We cannot assume that the research evidence we identified represents 'real world' practices, and therefore our claims for the transferability of this research must be guarded. 


\section{Introduction}

Nutrition is an important component of healthcare. It plays a critical role in the prevention and treatment of most cardiovascular and cerebrovascular diseases, which are leading causes of morbidity and mortality throughout the world ${ }^{1-3}$. Nutrition is even more important in sub-Saharan Africa because malnutrition is a major cause of morbidity and mortality, particularly among children ${ }^{4}$.

Several landmark reports ${ }^{5,6}$ have identified the delivery of nutrition care as one of the core responsibilities of doctors. Research has also shown that nutrition counselling delivered by them has positive influence on patients' clinical outcomes. They and other healthcare professionals whose primary role is not nutrition care, however, often miss opportunities to advise patients on diet and health ${ }^{7,8}$. Health workers in primary care settings are particularly important providers of nutrition care because they can motivate even healthy individuals to adopt healthier lifestyles ${ }^{9}$. The care expected from primary care health workers includes nutrition assessment, education and counselling interventions, monitoring, and evaluation. Lack of knowledge ${ }^{10}$, skills, and confidence ${ }^{11,12}$ as well as negative attitudes towards delivery of nutrition care and low outcome expectancy ${ }^{13}$, are barriers to healthcare professionals providing nutrition care. In addition to these individual-related factors, several system-related factors such as lack of time, office space, payment, materials, and education ${ }^{14}$ also prevent the delivery of nutrition care by these healthcare professionals.

Many educational interventions have been designed and implemented to improve nutrition care but their effects have been inconsistent and often weak ${ }^{15-17}$. There remains a need, therefore, for interventions that can change healthcare professionals' behaviour in practice ${ }^{15-17}$. It is imperative to identify contextual factors, which mediate or inhibit their competence and delivery of nutrition care ${ }^{18,19}$. In order to meet those needs, researchers have to identify components of effective educational interventions and processes.

To date, only one secondary research investigation has synthesised conclusions from existing evidence about nutrition care ${ }^{20}$. The authors of that review concluded that inservice nutrition training improved healthcare professionals' knowledge, nutrition-related counselling skills, and malnutrition management skills. The main limitation was that this was a traditional systematic review, which only considered in-service nutrition training programme. Its authors found that the evidence base was very heterogeneous; studies had widely varying study designs with heterogeneous outcome measures, and there were wide differences in the competence, experience, and backgrounds of participating healthcare professionals. As the authors acknowledged, systematic review methodology limited their ability to recognise and account for the complexity of interactions within such interventions.

We reasoned that we could move the field forward by conceptualising nutrition education interventions as complex ones within a realist research approach. As noted in our 
published review protocol ${ }^{21}$, we recognised that educational interventions involve multiple actors operating at different levels with a range of artefacts in varied material environments ${ }^{22}$. We assumed that these components operate in non-linear ways to yield context-dependent outcomes. Realist synthesis explores 'what is it about this intervention that works, for whom and in what circumstances?' and is therefore an appropriate way to study complex interventions ${ }^{23}$. It is an iterative, theory-driven approach, which aims to unpack the theories that inform decisions and actions adopted in the design and implementation of interventions ${ }^{24}$. Realist synthesis begins with the development of an initial programme (or candidate) theory about how interventions work, the contexts in which they do and do not work, and the differentiated patterns of outcomes that they generate ${ }^{25}$. As the review progresses, researchers test the initial programme theory and refine it as more evidence becomes available ${ }^{26}$.

Thus, the aim of this realist review was to determine what, how, for whom, why, and in what circumstances educational interventions to improve the delivery of nutrition care by doctors and other healthcare professionals work?

\section{Methods}

VM is a nutritionist working in sub-Saharan Africa, which provided a context for the research. Other members of the team included scholars of medical education, evidence synthesis, social science, nutrition, and an experienced clinician.

\section{Alteration from protocol}

The review question above is broader than in the published protocol ${ }^{21}$ because the searches showed important findings from research in health professions other than medicine, which the team felt could make a valuable contribution.

\section{Search methods}

Search terms pertaining to nutrition, care, healthcare professionals, training etc were scoped on two electronic databases. Resulting articles were reviewed and refinement of search terms was not considered necessary. Further explanation and a full list of the search terms can be found in our published protocol [21]. A search strategy was created for Ovid MEDLINE (available in online supplementary appendix 1) and adapted for the rest of the databases. These databases were CINAHL, ERIC, EMBASE, PsycINFO, Sociological Abstracts, Web of Science, Science Direct and Google Scholar, the latter of which was used to search for grey literature. Email alerts were set for journals and RSS feeds for databases to ensure that we identified new papers as soon as they became available. Email alerts were set for journals and RSS feeds for databases to ensure that we identified new papers as soon as they became available. 


\section{Inclusion criteria}

- Study participants: Medical students, students of other health professions and practising healthcare professionals (e.g, nurses, physician assistant, etc).

- Focus of intervention: Developing participants' competencies in any aspect of nutrition practice behaviour.

- Study design: All.

- Context of intervention: Medical schools, residency and fellowship programmes and interventions at community and hospital settings

- Publication language: English.

- Publication date: January 1994 to December 2014 inclusive. This date range was chosen because preliminary searches indicated that educational interventions to improve nutrition care competencies and delivery among doctors and other healthcare professionals gained prominence within published literature around 1994.

\section{Exclusion criteria}

We sought to understand generalists' delivery of nutrition education, and therefore we excluded research that only considered the education of dietitians and/or nutritionists since nutrition is their main responsibility. While we excluded conference proceedings, opinion pieces, case studies and abstracts, we used them to develop the initial candidate theories reported in ourprotocol ${ }^{21}$. We also excluded systematic reviews, although they informed the design of our data extraction form and provided an insight into context, mechanism, outcome (CMO) configurations and additional references. Papers were also excluded if they lacked evaluation or outcome data and not being about improvement in nutrition care competencies.

\section{Study selection}

Figure 1 shows the flow chart of the search and selection processes. The final search yielded 4500 hits.VM and TD initially screened the titles of 100 hits independently and compared their findings. There was almost complete agreement and VM continued with the screening. After eliminating duplicates, 357 studies were selected. Having obtained their abstracts, VM, TD, and MGC determined independently whether each study was concerned with improving nutrition care competencies and delivery of nutrition care. At a face-to-face discussion, we compared our choices, for which the $k$ statistic of agreement was 0.9. This yielded 74 studies, 6 of which were excluded because they were conference abstracts. Seven studies could not be obtained despite repeated attempts. VM read the reference lists of the remaining 61 studies and all identified systematic reviews, identifying 11 more studies. 


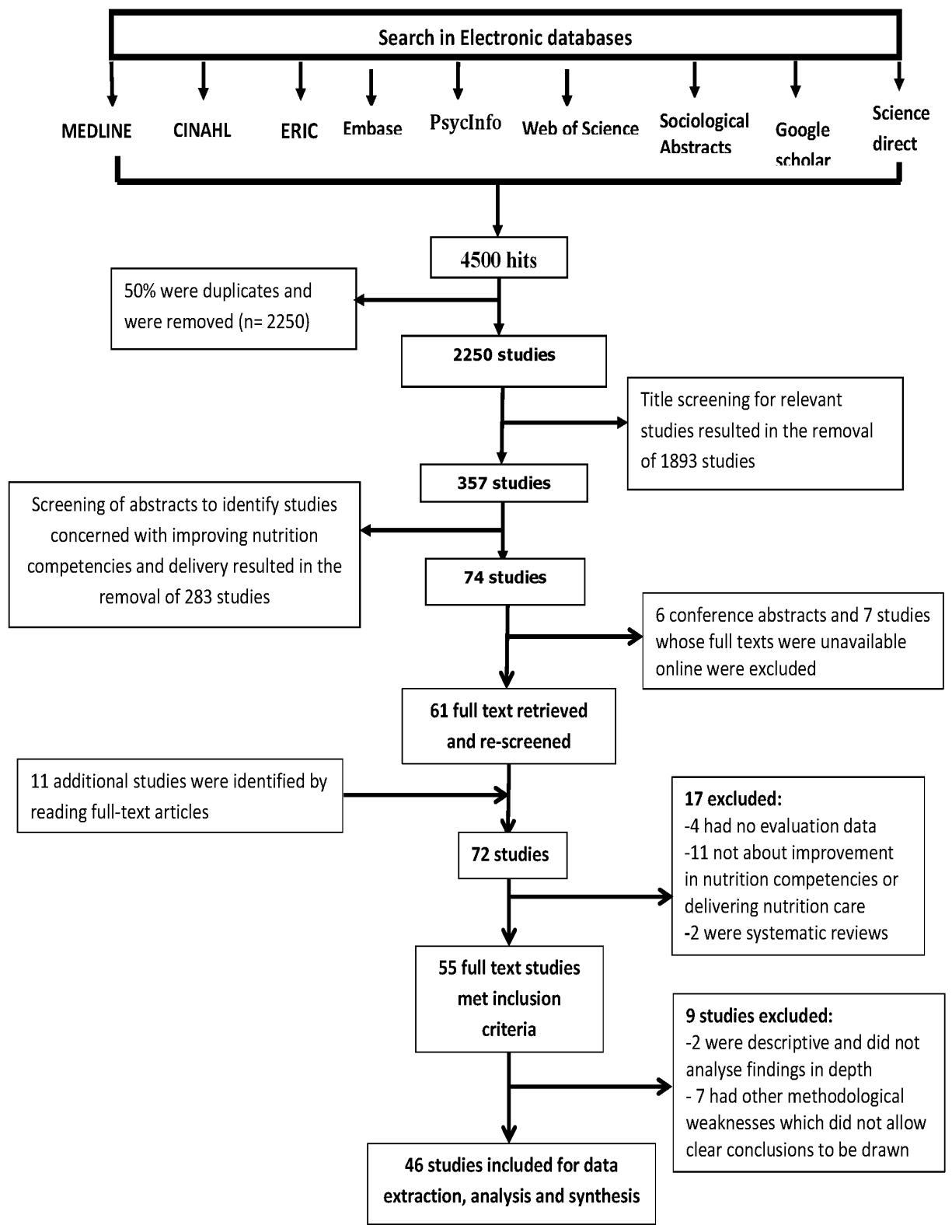

Figure 1: Flow chart of search and selection process

\section{Quality assessment}

It is regarded as acceptable in realist synthesis to include part(s) or whole studies for analysis and synthesis, provided that the methods employed for collecting such data are robust ${ }^{27}$.As recommended by Pawson ${ }^{25}$, the appraisal of primary studies was informed by 
their relevance as well as their rigour ${ }^{25}$. Our judgement of a study's relevance were informed by the extent to which the whole study or parts of it were relevant to our published initial programme theory ${ }^{21}$. Our assessment of rigour was informed by the trustworthiness of studies' design, sample size and data collection tools in relation to the outcomes reported. The Mixed Methods Appraisal Tool ${ }^{28}$ helped us assess rigour ${ }^{28}$. Based on the exclusion and inclusion criteria, VM selected 55 of the 72 studies for quality assessment. Quality assessment was conducted by AS, TD, FS and MGC. This process resulted in the exclusion of nine studies from which clear conclusions could not be drawn because of methodological weaknesses. The remaining 46 studies were included into our data analysis. We kept notes of our reasons for including or excluding each study and resolved doubts about our judgements of study quality by discussing between ourselves. The processes of quality assessment and data extraction proceeded concurrently.

\section{Data extraction, analysis and synthesis}

For the purpose of data extraction, we followed guidance from previous related systematic reviews ${ }^{20,22,29-31}$ and iteratively refined our procedures in accordance with the focus of the review. VM initially extracted data from a sample of 10 studies, discussed the findings with the other members of the team and used those discussions to guide further data extraction. Data extracted included the following:

- Study design, sample size and outcome data

- Educational levels of study participants (students vs practising health workers)

- How course material had been developed

- Topics covered

- Methods of teaching and learning

- Methods of evaluating outcomes including data collection tools

- Intervention type (e.g, workshops, curriculum designs)

- Durations of intervention

- Contexts of intervention (e.g, practising healthcare professionals, students)

- Mechanisms generated

- Learning outcomes

- Impacts (if any) of intervention on clinical outcomes

- Any theories or mechanisms postulated by author(s) explaining the effects of interventions

We read all 46 included studies twice, transferring relevant data into our data extraction form. We identified the CMOs and interactions between them for each study as well as the theory informing each intervention. To do that, we assumed that the design of each study was informed by a theory, which the authors stated explicitly or implicitly. Identify- 
ing those theories helped us understand how interventions worked to generate outcomes. We discussed and reflected on all the data we had identified for each study, sometimes using extracts of publication narratives to foster reflection.

The next stage was to identify themes that were common to different studies. Using an interpretative and narrative approach, we discussed and synthesised initial conclusions, which we used to refute or refine the candidate theories in our published proto$\mathrm{col}^{21}$. We chose this process of synthesis in preference to a meta-analysis, which would not have been possible given the diversity in study populations, designs, interventions, and outcomes ${ }^{32}$.

\section{Results}

\section{General characteristics of the studies}

Table 1 provides a summary of the 46 studies. Twenty-seven (59\%) came from the USA, 7 (19\%) from Europe, 4 each from South America (all from Brazil) and Asia, 2 from Canada and 1 each from Africa (i.e, South Africa) and Australia. In total, 4816 participants participated in them (median=76 participants; IQR: 47, 178). Interventions that had healthcare professionals as participants had somewhat larger numbers (median=98; IQR: 46, 163) than those having students as participants (median=54 participants; IQR 32, 152).

The studies had varied study designs (shown in Table 2 ) with a preponderance $(n=39$, 85\%) of quasi-experimental designs. Twenty-one studies had follow-up evaluations after the pre-test and post-test evaluations. The time period between post-test and follow-up evaluations ranged between 2 weeks and 12 months.

Most studies ( $n=32,70 \%$ ) evaluated outcomes using surveys of knowledge, attitudes, self-reported practice behaviours, self-efficacy, confidence, and feedback. A large proportion of these surveys were developed by the authors, who did not usually report the psychometric properties of their instruments. All the interventions that set out to improve knowledge used multiple-choice questions (ranging between 1 and 78 questions). Changes in attitude before and after interventions were assessed using the Likert scales, anchored with statements describing attitudes.

Most questionnaires measuring behaviour changes used self-reported changes in nutrition practice behaviour. A few studies observed clinical behaviour to measure changes in nutrition practice. For example, one study in the Netherlands ${ }^{33}$ used incognito standardised patients to assess the impact of an intervention on the nutrition practice behaviour of general practitioner (GP) residents. Another study in Brazil ${ }^{34}$ measured nutrition indices (i.e. wasting, stunting, and underweight) of children to determine the impact of an educational intervention that aimed to improve the provision of nutrition counselling to mothers and/or caregivers by doctors. 


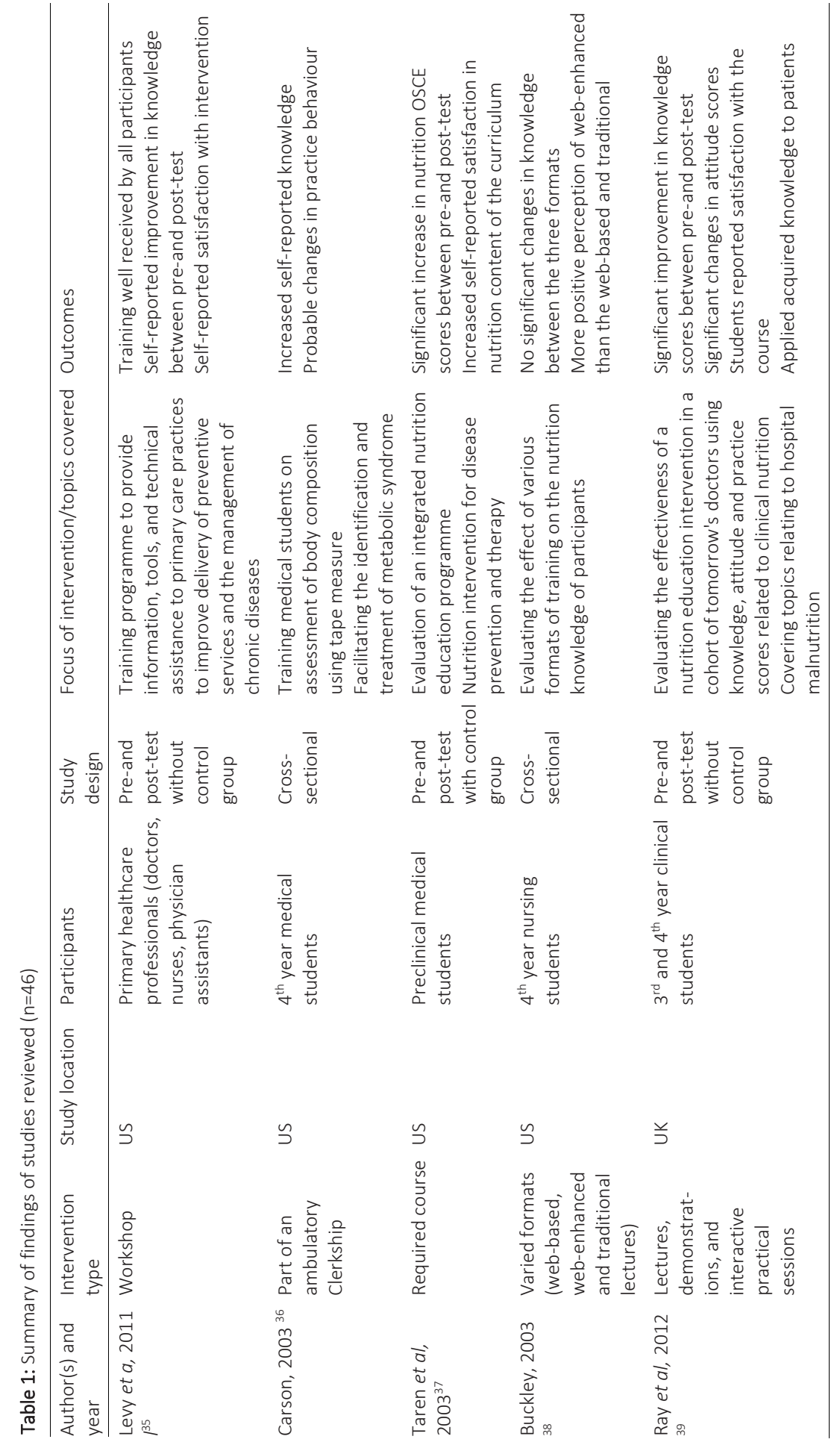




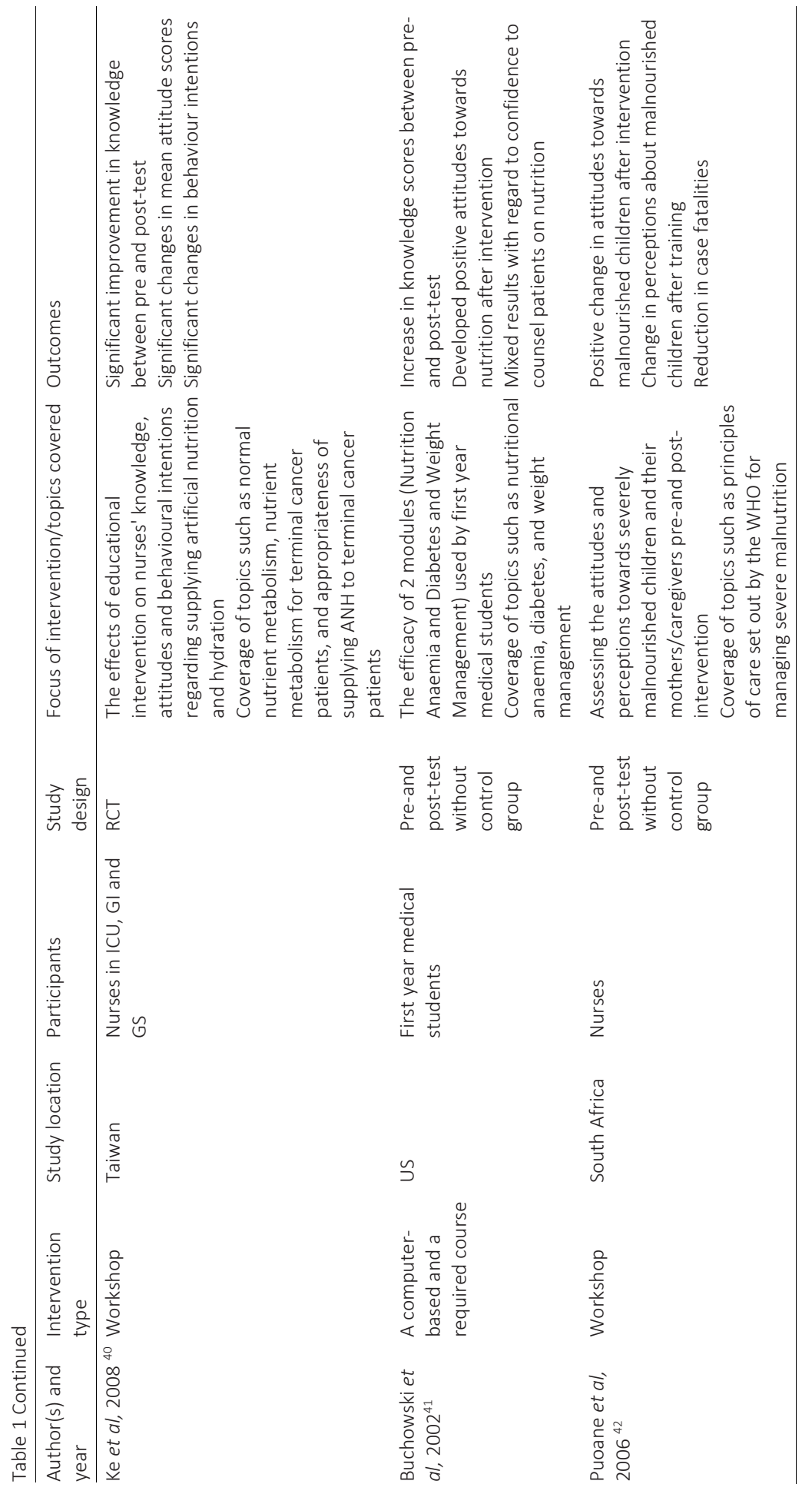




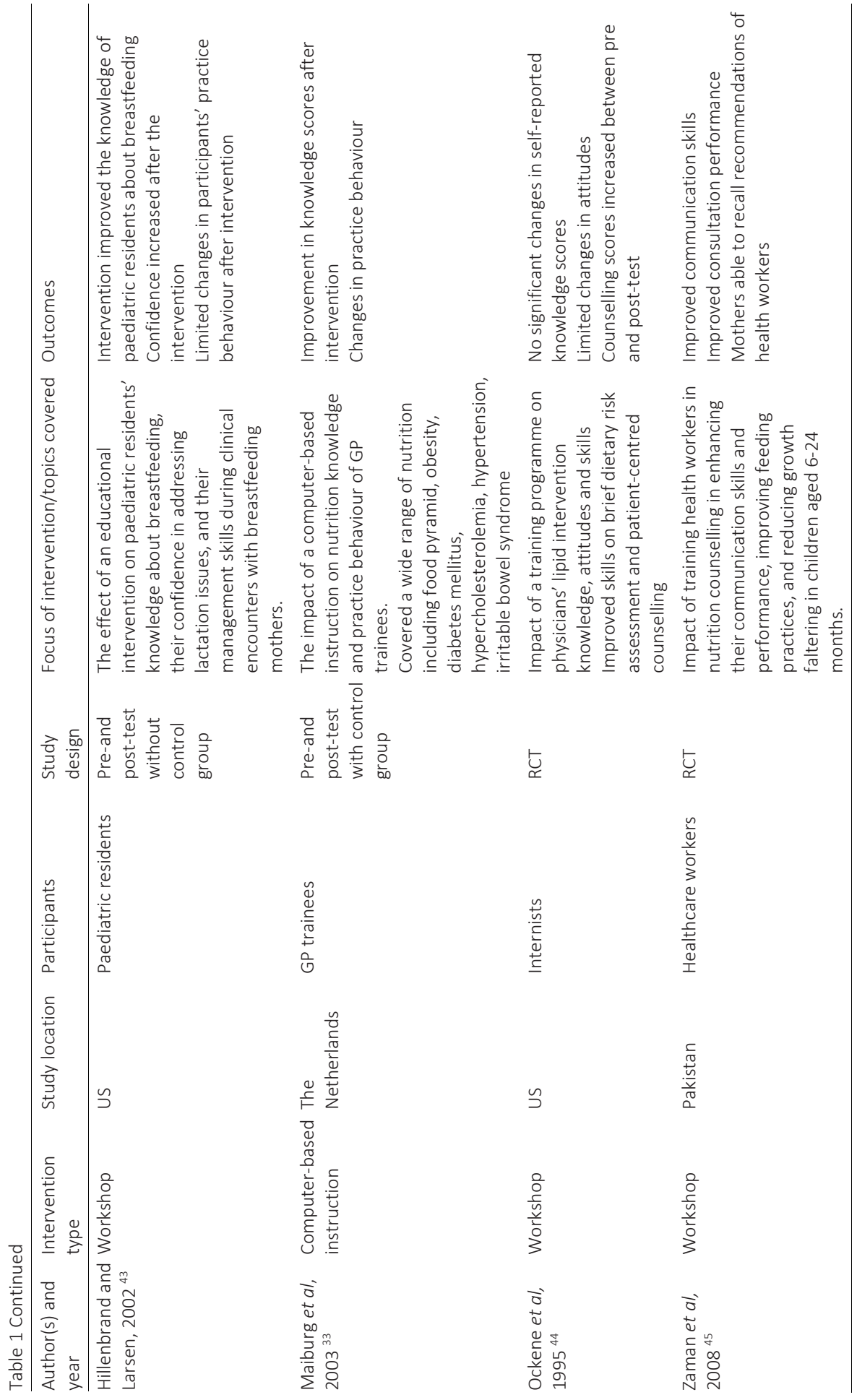




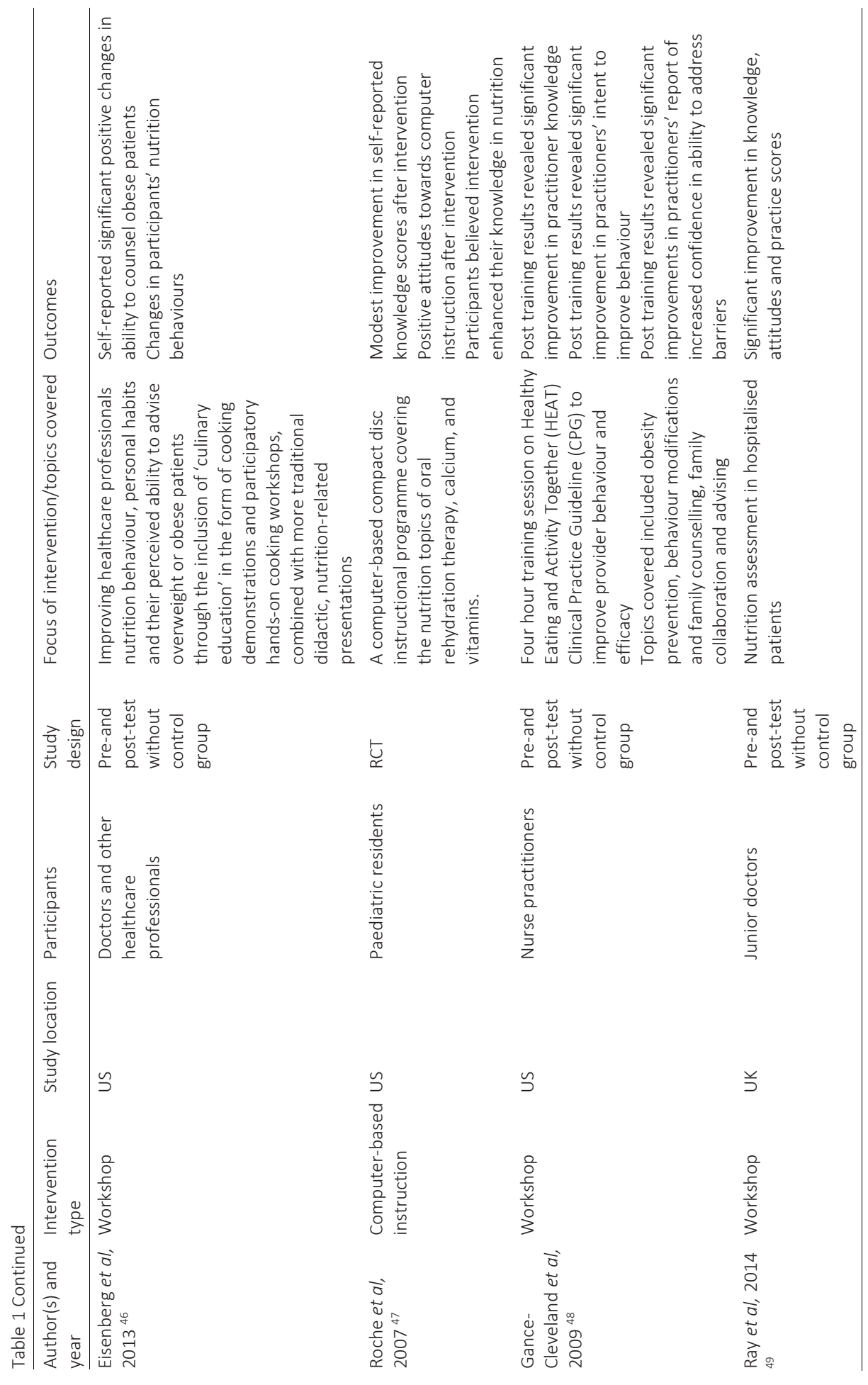




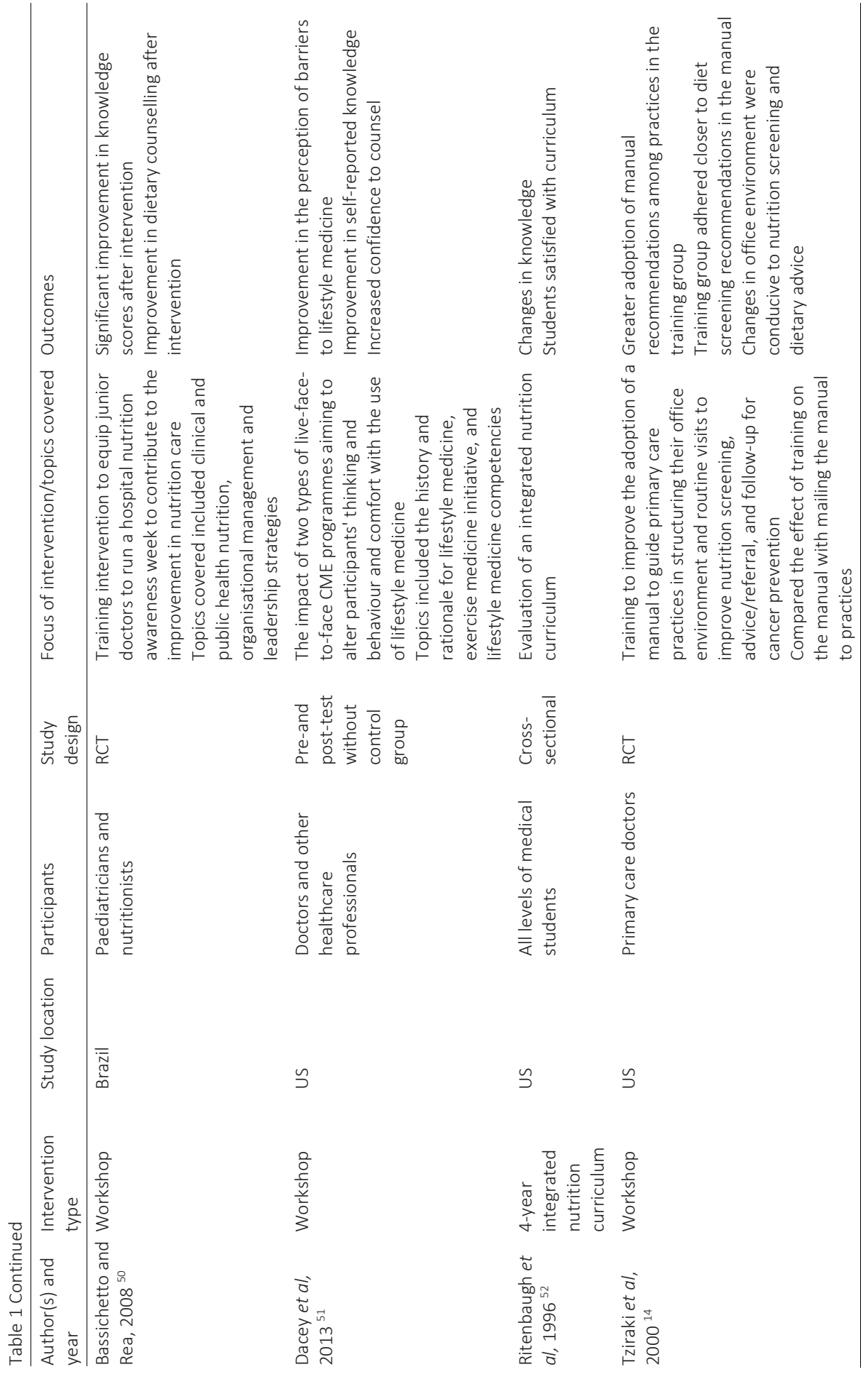




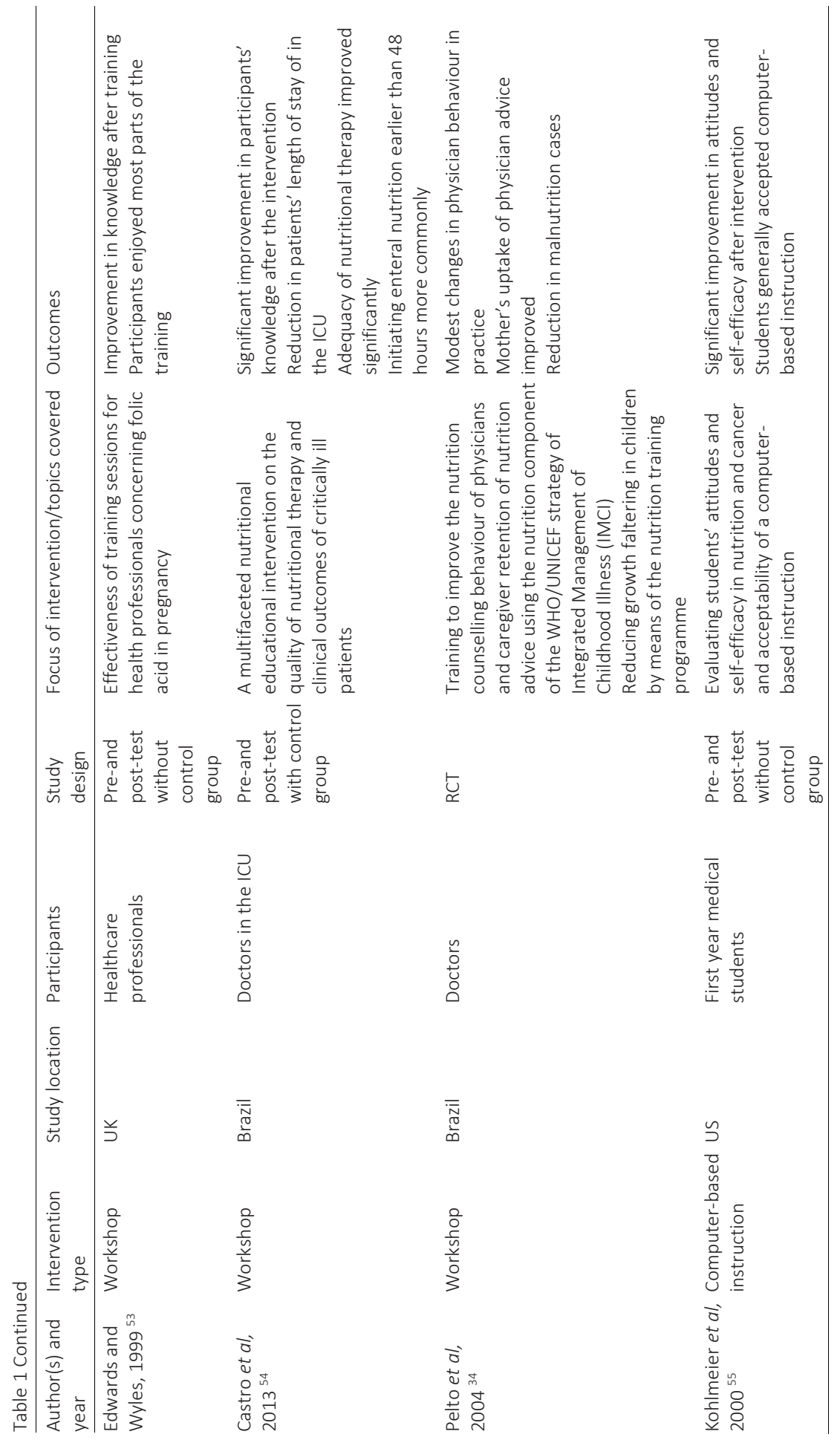




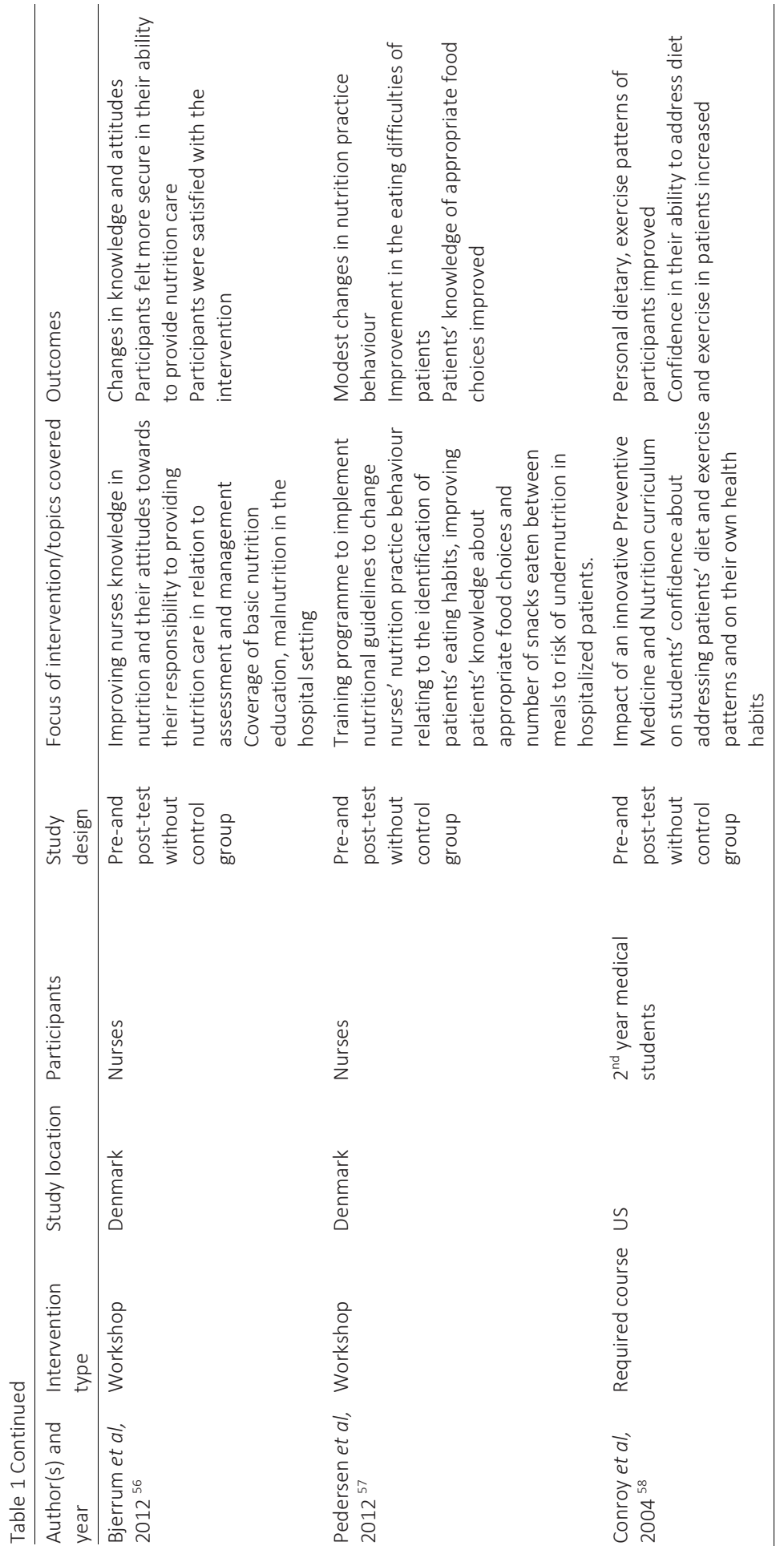




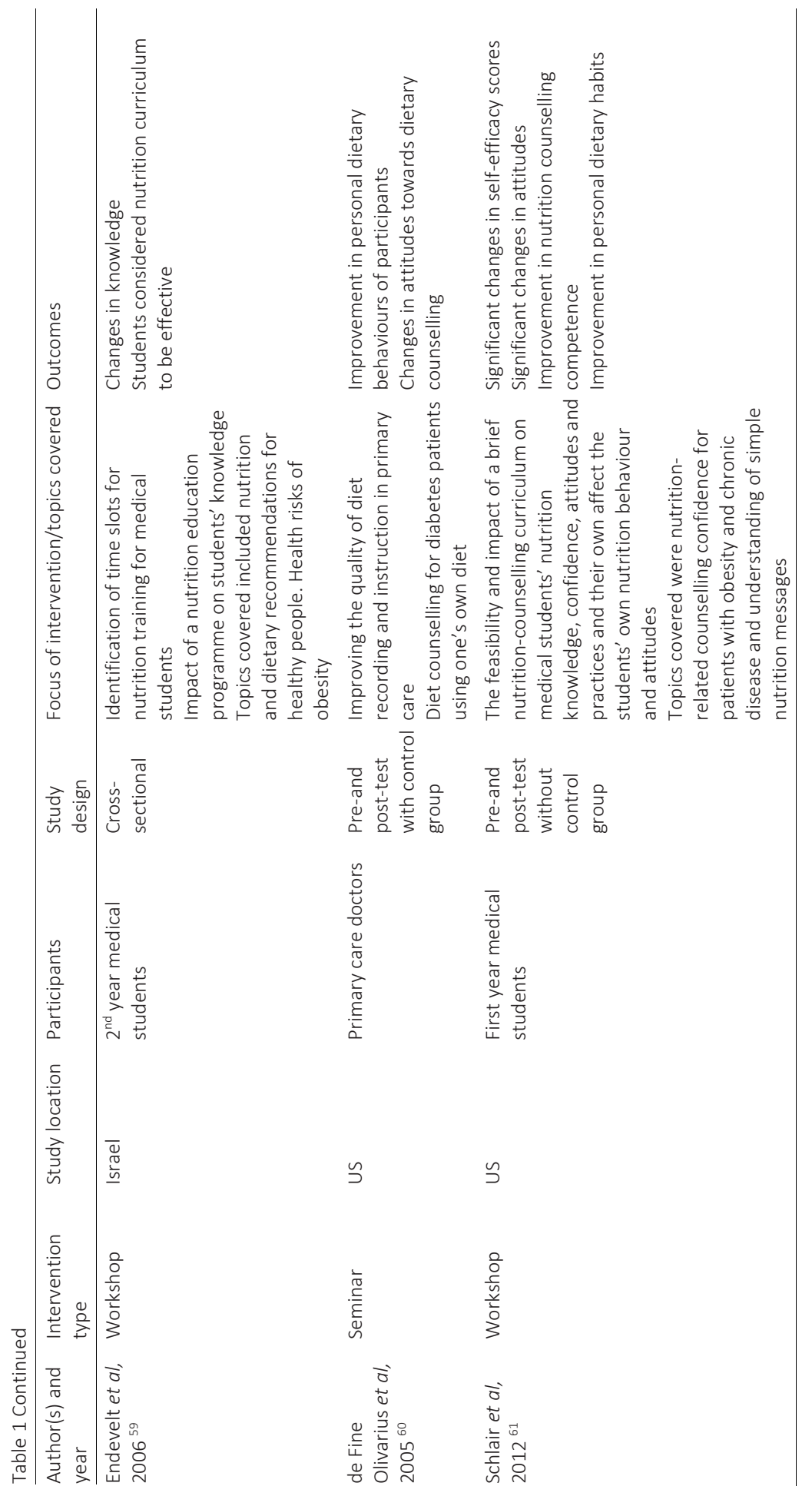




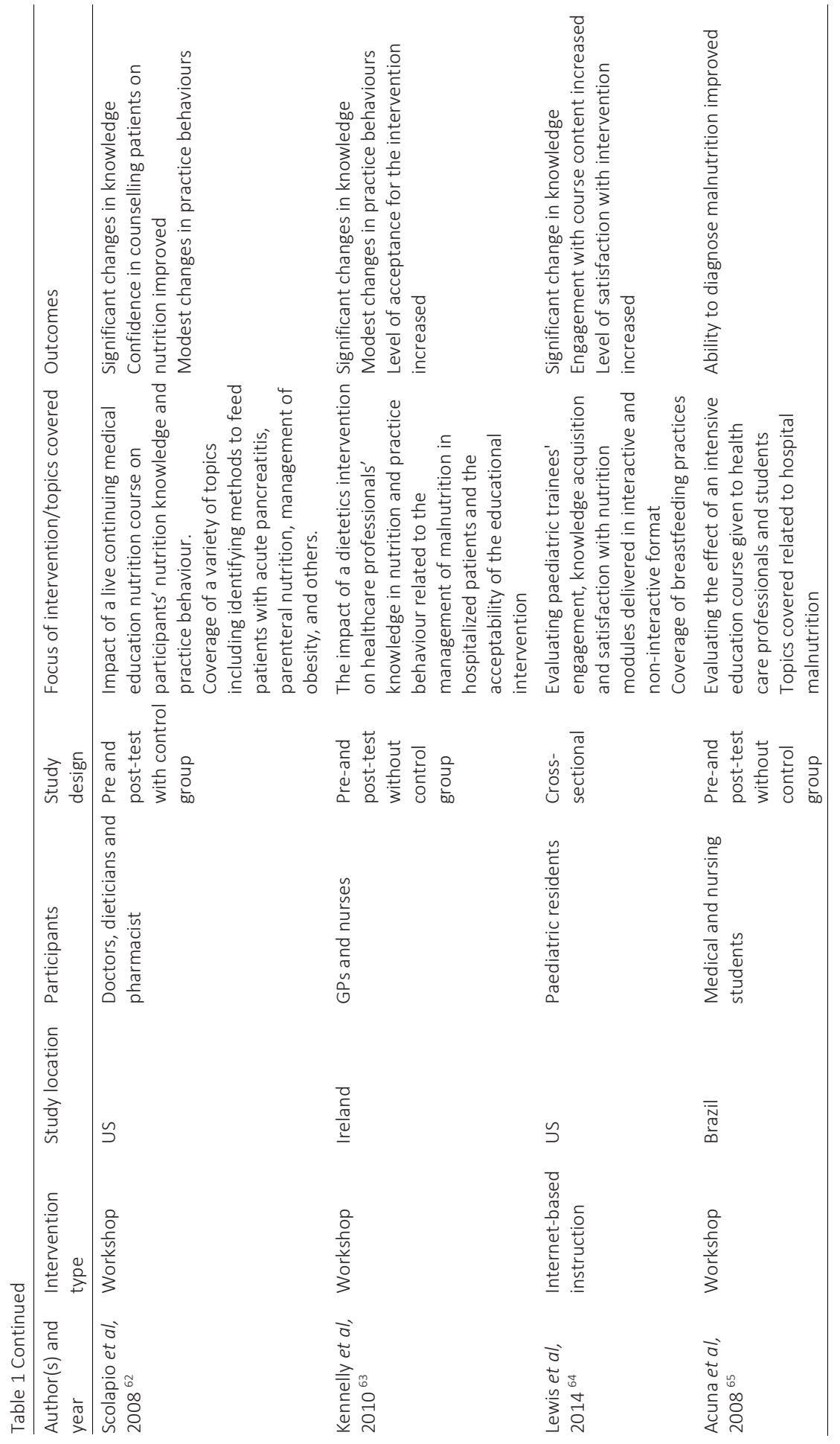




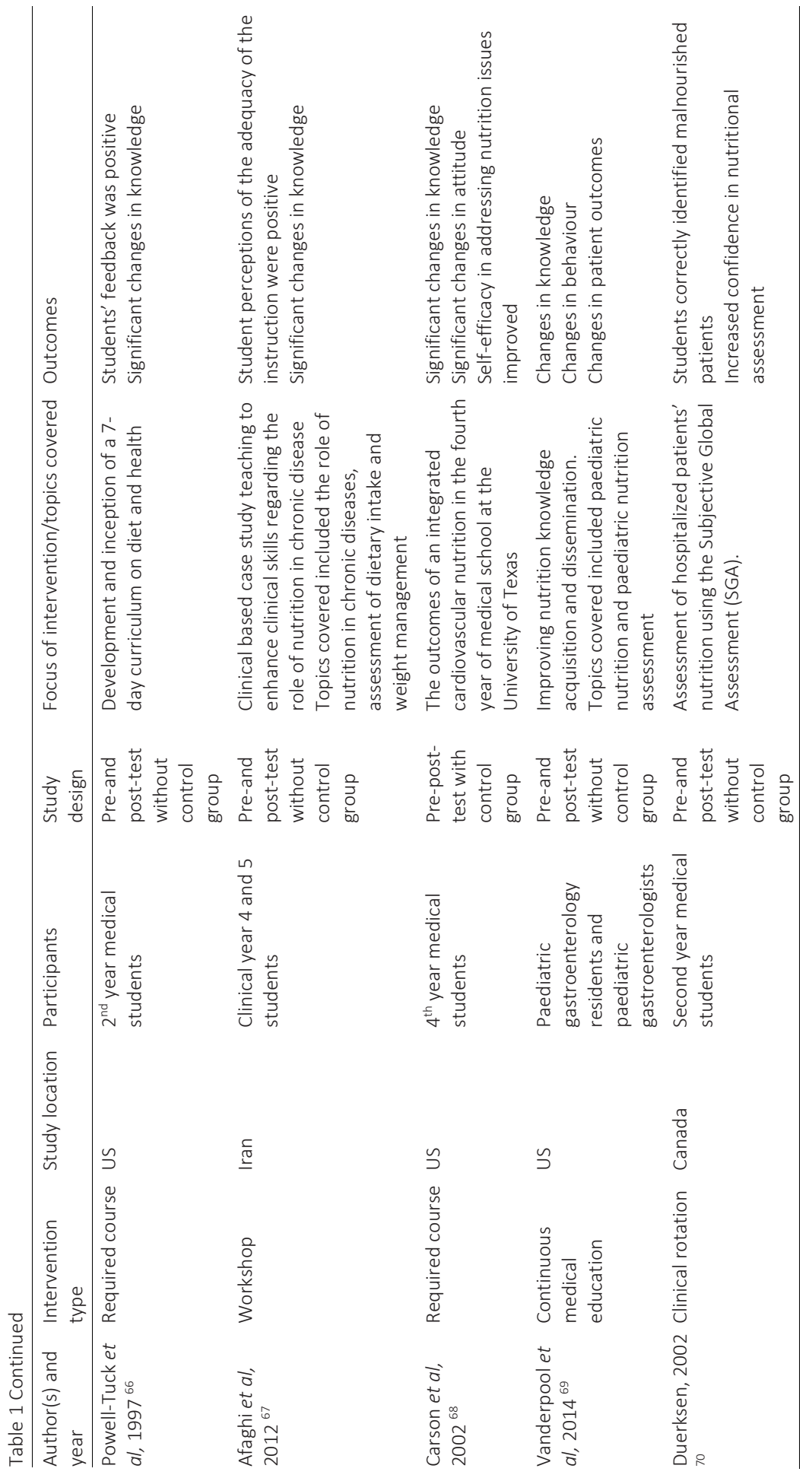




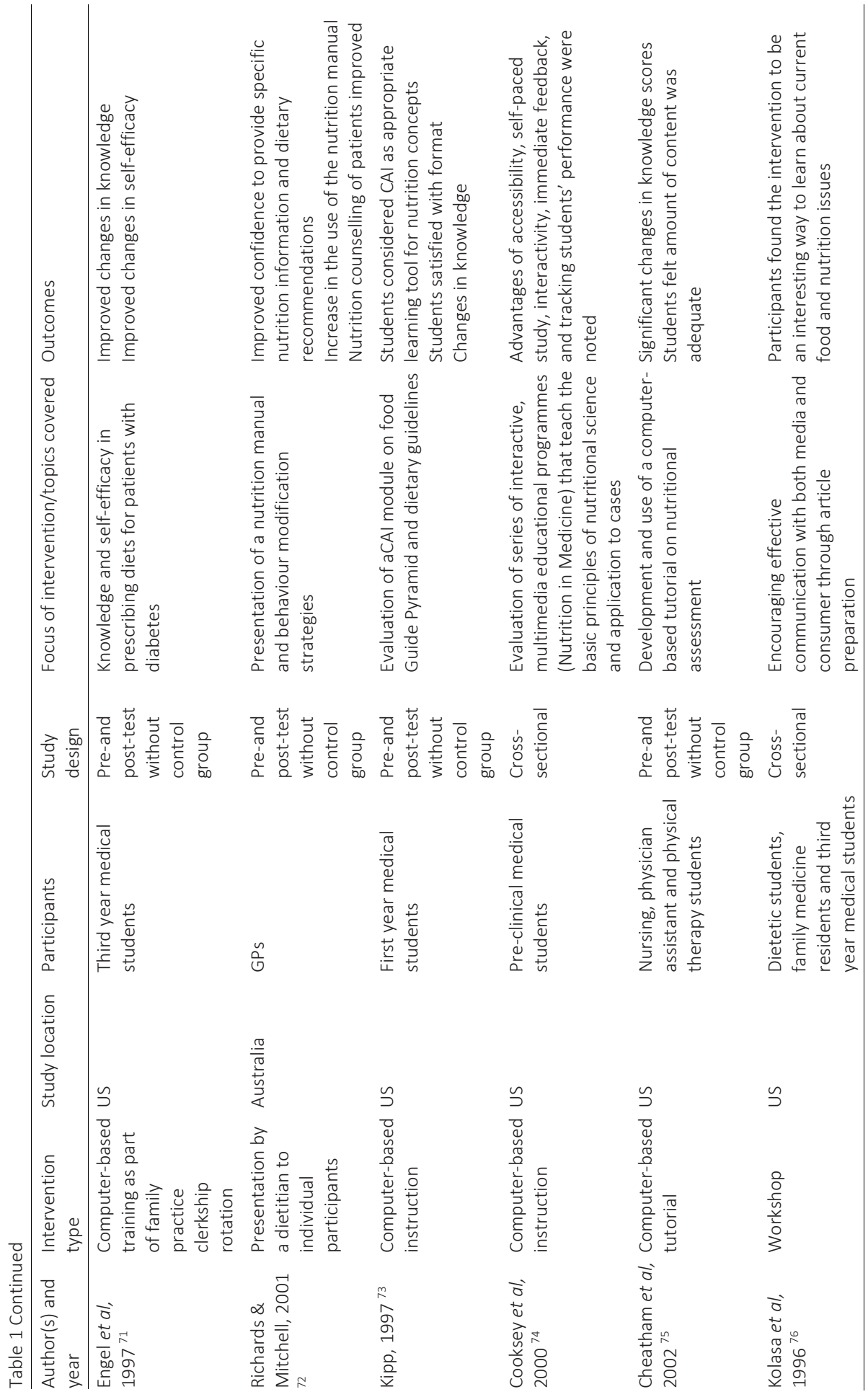




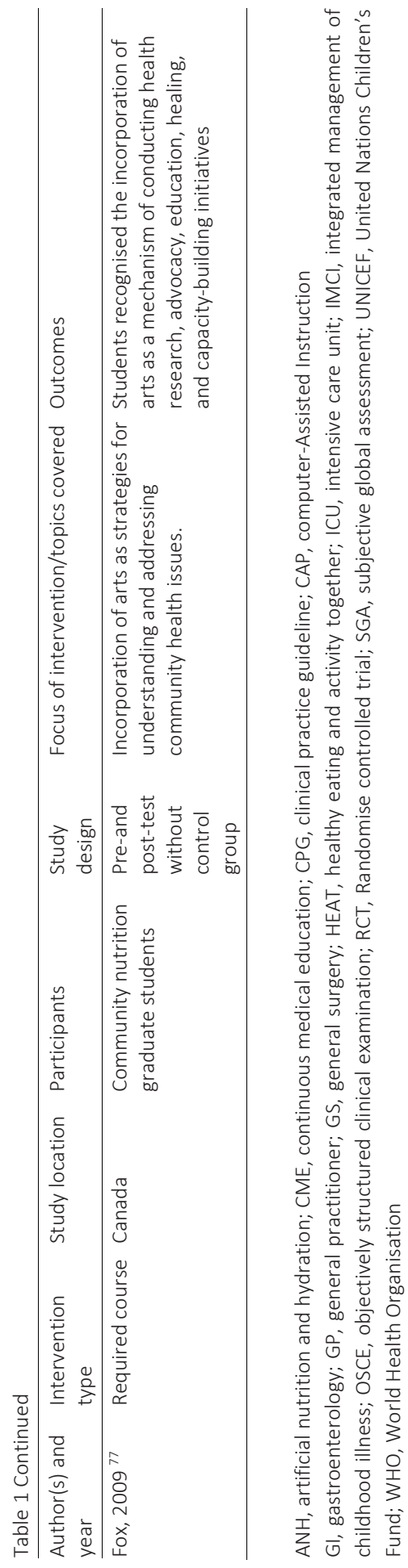


Intervention focus, types, teaching and learning formats, duration of interventions and expected learning outcomes

Only 11 studies (24\%) explicitly stated the theoretical underpinning of their interventions. These included experiential, social, and cognitive learning theories as well as cognitive theory of multimedia learning. The purpose of most interventions was to improve participants' competencies (i.e, knowledge, skills and attitudes) in a variety of nutrition topics (shown in Table 1). Studies originating from developing countries tended to cover topics related to infant and young child feeding practices, whereas those from developed countries covered topics related to hospital malnutrition and nutritional management of chronic diseases. Most studies in which students participated aimed to increase curriculum contact hours and nutrition content. Studies involving practitioners were usually continuous medical education (CME) programmes aiming to improve knowledge, attitudes, skills, and practice behaviour in specific topics such as breastfeeding practices and dietary counselling. Teaching and learning formats included lectures, problem-based learning tutorials, nutrition slogans, demonstrations, role plays, group discussions, games, and video presentations. All interventions used more than one teaching and learning format except six, which were either lecture based or computer based only ${ }^{33,40,47,55,64,69}$. Almost all of the interventions used innovative teaching and learning methods. Interventions involving students were usually obligatory and lasted from between 2 weeks to 4 years. Those involving professionals were generally shorter. The shortest intervention was a 1 hour intensive session for GPs and other healthcare professionals on the benefits of giving folic acid to women of child bearing age ${ }^{53}$; the longest were two 4-year integrated nutrition curricula for medical students ${ }^{37,52}$. Inconsistent reporting of the length of interventions (including use of terms like credit hours) made it difficult to determine their average lengths. 
Table 2: Study designs and data collection methods

\begin{tabular}{|c|c|}
\hline Characteristic & Frequency (\%) \\
\hline \multicolumn{2}{|l|}{ Study design } \\
\hline Randomised control trials & $7(15 \%)$ \\
\hline \multicolumn{2}{|l|}{ Quasi-experimental } \\
\hline Pre-test-post-test with control group & $6(13 \%)$ \\
\hline Pre-test-post-test without control group & $26(57 \%)$ \\
\hline Cross-sectional & $7(15 \%)$ \\
\hline \multicolumn{2}{|l|}{ Methodological approach } \\
\hline Qualitative & $5(10 \%)$ \\
\hline Quantitative & $32(70 \%)$ \\
\hline Both qualitative and quantitative & $9(20 \%)$ \\
\hline \multicolumn{2}{|l|}{ Data collection method } \\
\hline Questionnaires/surveys only & $32(70 \%)$ \\
\hline Observations only & $2(4 \%)$ \\
\hline Focus group discussions only & $2(4 \%)$ \\
\hline Questionnaires/survey with other methods (e.g. interviews, observations) & $10(22 \%)$ \\
\hline \multicolumn{2}{|l|}{ Format of intervention } \\
\hline Training programmes & $12(26 \%)$ \\
\hline Workshops & $9(20 \%)$ \\
\hline Required courses & $7(15 \%)$ \\
\hline Technology-based (computer-based, internet-based) & $11(24 \%)$ \\
\hline Ambulatory clinical rotations & $2(4 \%)$ \\
\hline Seminars & $1(2 \%)$ \\
\hline Continuing medical education programmes & $4(9 \%)$ \\
\hline \multicolumn{2}{|l|}{ Healthcare professionals ( $n=22,48 \%$ ) } \\
\hline Doctors (general practitioners/primary care) & $8(36 \%)$ \\
\hline Nurses & $5(23 \%)$ \\
\hline Multidisciplinary participants (e.g. nurses, doctors, pharmacists) & $9(41 \%)$ \\
\hline \multicolumn{2}{|l|}{ Students ( $n=24,52 \%)$} \\
\hline Undergraduate, preclinical & $14(58 \%)$ \\
\hline Undergraduate, clinical & $5(21 \%)$ \\
\hline Postgraduate & $5(21 \%)$ \\
\hline
\end{tabular}

GPs, General Practitioners

\section{Context-mechanisms-Outcomes configurations}

Table 3 lists the CMOs identified from the included studies. We describe here how those interacted to yield CMO configurations. 
Emphasising skills development instead of knowledge outcomes ('Let me be skilful')

Researchers were often triggered to design interventions by professionals' lack of knowledge about nutrition. This was particularly true of undergraduate education $38,41,59,64,66,67$, where all but one ${ }^{53}$ of the interventions primarily targeting knowledge took place. Yet interventions that only aimed to improve knowledge were less likely to change practice behaviour. In four studies, for example, significant gains in knowledge did not predict practice scores ${ }^{39}$, improve students' assessment of the nutrition status of overweight patients ${ }^{36}$, influence behaviour change intentions ${ }^{40}$, or affect dietary counselling for mothers/caregivers of children aged $12-24$ months $^{50}$. Furthermore, there were interventions, which did not significantly increase knowledge yet changed behaviour. For example, a significant improvement in diet counselling during physician-patient interactions 44,51 and increased self-reported counselling behaviour and confidence ${ }^{51}$ took place without any significant increase in knowledge. In one study, Ockene et al ${ }^{44}$ noted that 'a large proportion (1.5hour) of the entire 3-hour CME training programme was devoted to the learning of counselling and dietary assessment skills'. These findings show that it is important to train skills and create learning environments that encourage the acquisition of skills in order to change healthcare professionals' nutrition care behaviour ${ }^{78,79}$. 
Realist synthesis of educational interventions to improve nutrition care competencies

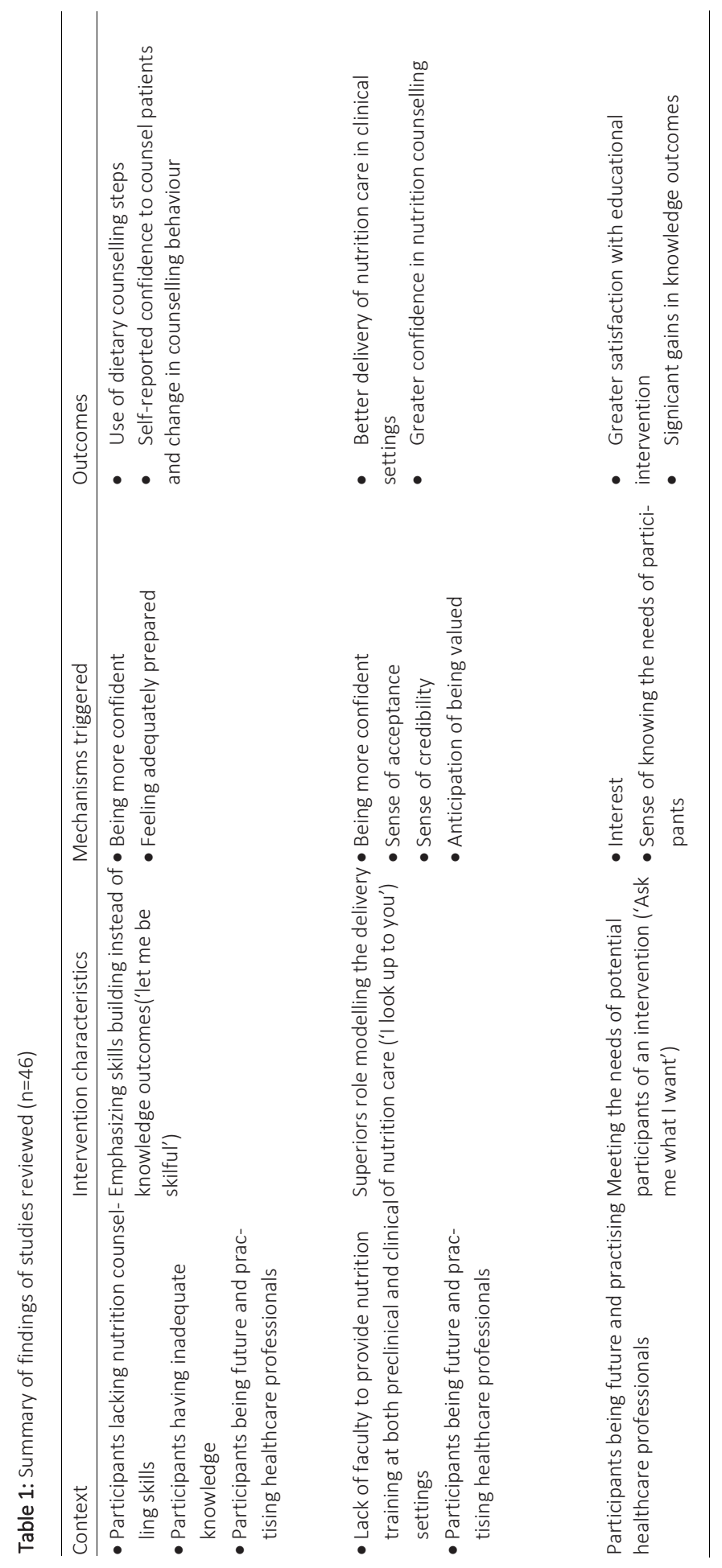




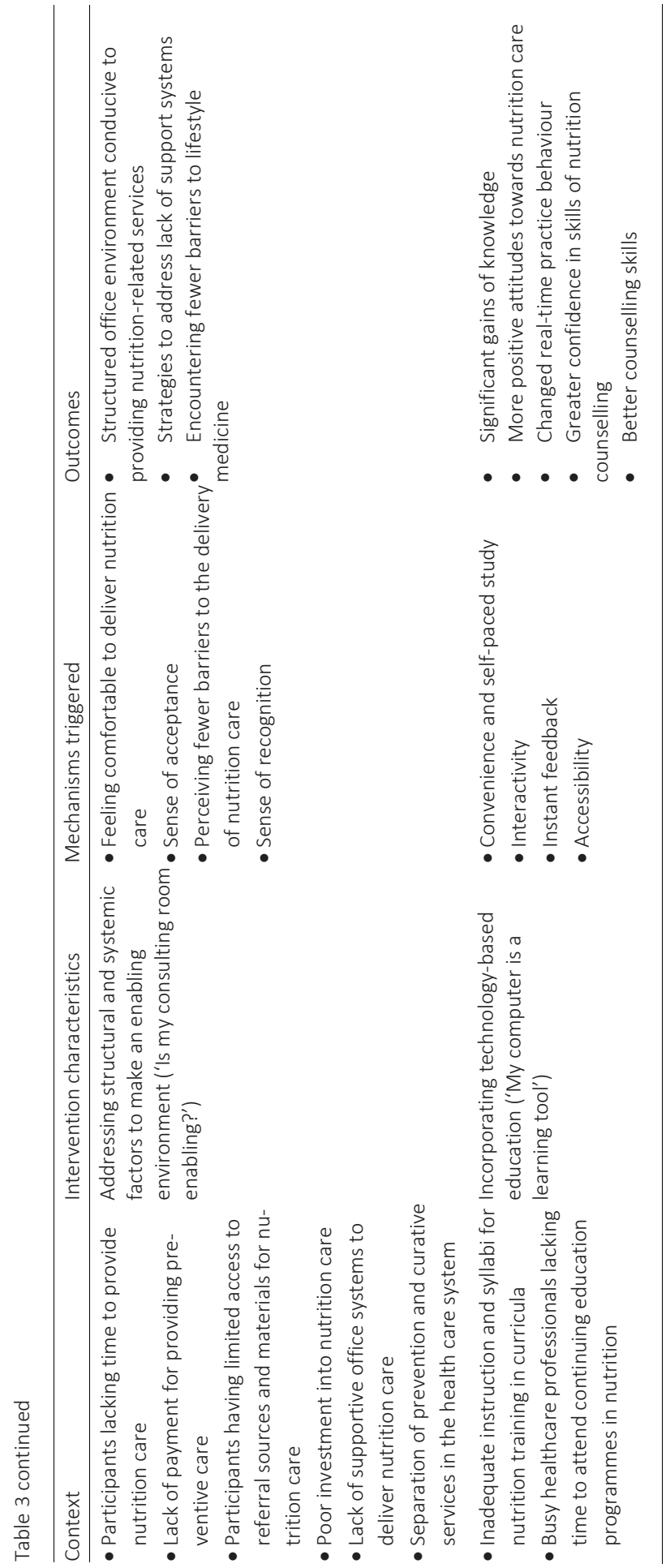




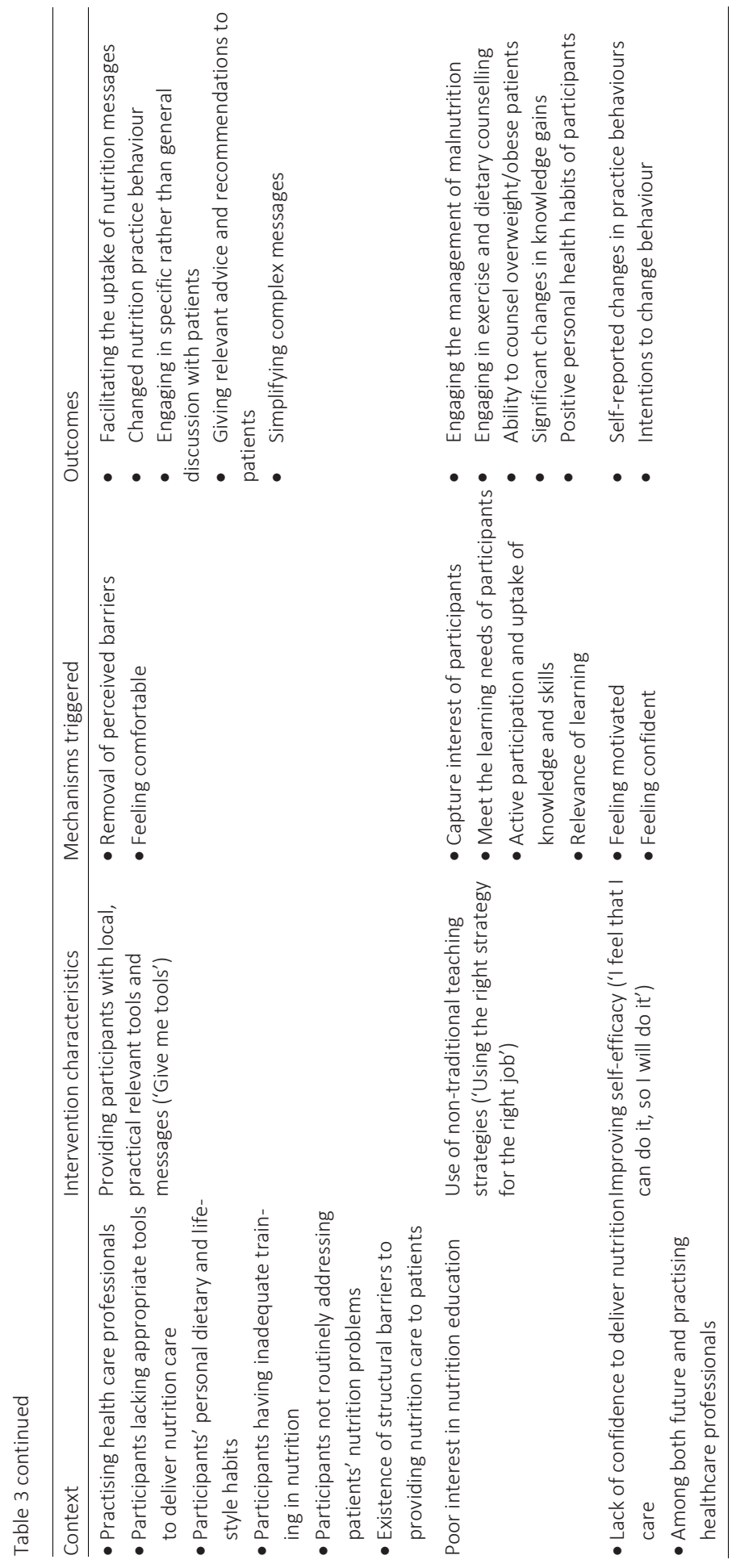




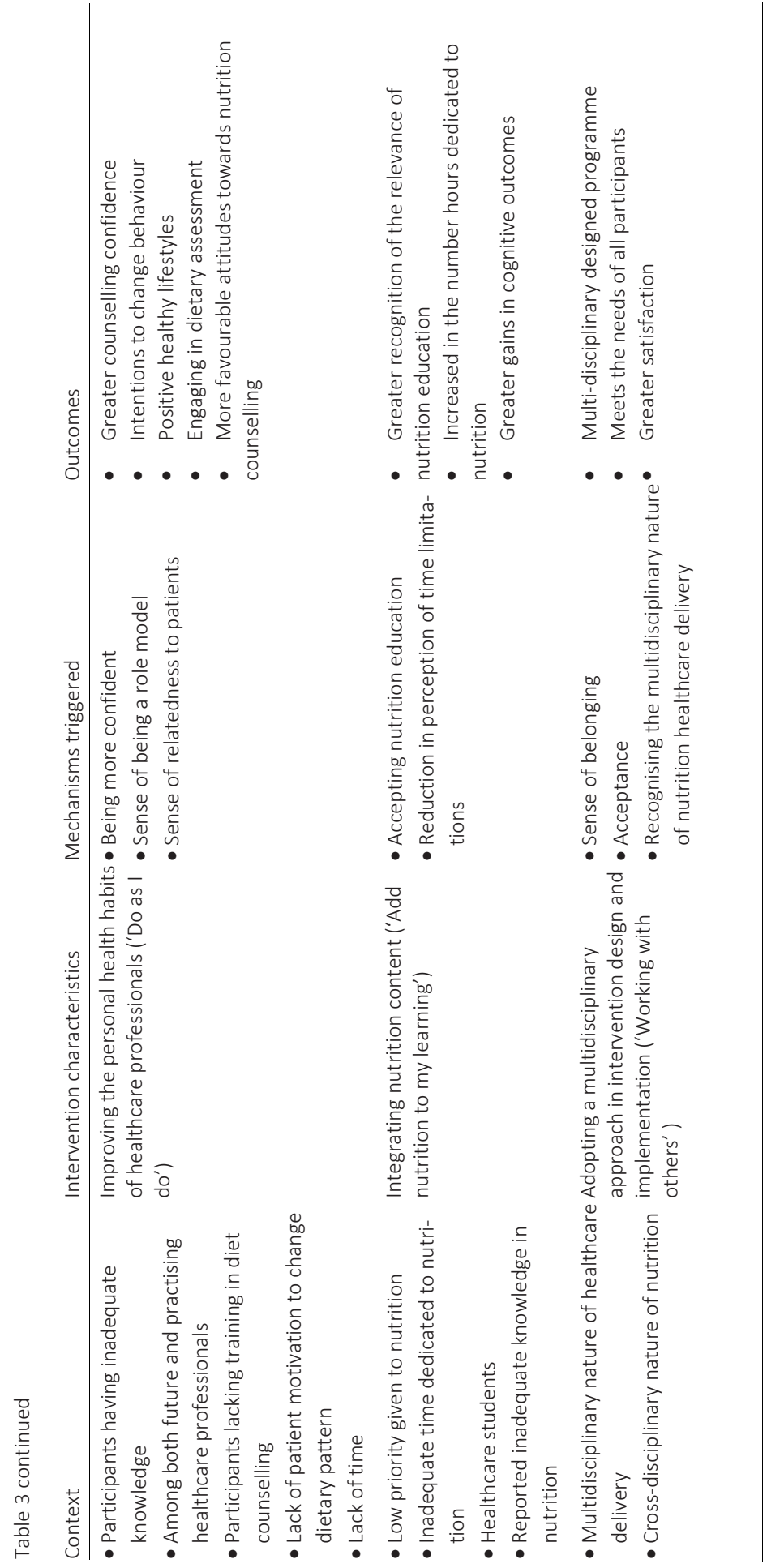




\section{Superiors role modelling the delivery of nutrition care ('l look up to you')}

A candidate theory in our published protocol ${ }^{21}$, that healthcare professionals would be more likely to deliver nutrition care if they saw their superiors model the same behaviour, was apparent in the evidence. Seeing superiors model nutrition care led research participants to feel more confident, accepted, and credible. They anticipated their actions being valued, which led them towards changing their nutrition practice. Virtual physician mentors ${ }^{41}$, simulation of GP consultations using video clips $^{33}$, physicians describing how they addressed nutrition in practice ${ }^{68}$, and role modelling by physicians in classes $^{68}$ were among the interventions that provided positive role modelling.

\section{Meeting the needs of potential participants of an intervention ('Ask me what I want')}

Most interventions were modelled on the theory that education will be most successful when it is designed to meet participants' needs $35,36,39,49,51,53,59,62,63,67-69$. Assessment of needs identified gaps in learners' knowledge or practice behaviour ${ }^{62}$, and how they learned best. It informed the content, format, and design of curricula. It helped select teaching and learning methods to which participants were receptive, which they found interesting and satisfying, and which led them to value their education.

\section{Addressing structural and systemic factors ('Is my consulting room enabling?')}

As well as education, interventions that improved working environments influenced participants' behaviour and helped maintain changes that had been achieved ${ }^{57}$. Eight studies helped participants address lack of support ${ }^{35,48,49,69}$ and systematic barriers ${ }^{14,34,51,57}$. They restructured office environments to make them more conducive to providing nutrition care ${ }^{14}$. Pelto et $\mathrm{al}^{34}$, for example, stated that 'structural conditions in the public health system in Pelotas provided an environment in which physicians could utilize their knowledge' (p360). Other researchers provided nutritional messages that busy primary care providers could deliver to patients ${ }^{35}$. Presentations on change management and leadership ${ }^{49}$ and provision of guidelines on office organization ${ }^{14}$ helped improve nutrition care. Collaboration between education and care delivery leaders helped remove structural and systemic barriers ${ }^{30}$. These created working environments that were conducive to the delivery of nutrition care.

\section{Incorporating technology-based education ('My computer is a learning tool')}

Seven studies used technology to resolve challenges relating to healthcare professionals having insufficient time to attend continuing education programmes, programmes having inadequate nutrition content, and faculty being unavailable to teach $33,41,47,55,64,68,74$. Computer-based and internet-based interventions allowed easy updating of content ${ }^{33}$, per- 
mitted self-directed and independent study of nutrition information ${ }^{33,47}$, presented content consistently ${ }^{68}$, were accessible ${ }^{74}$, promoted interactivity ${ }^{74}$, and were convenient for participants because they were self-administered ${ }^{33,68}$ and self-paced ${ }^{74}$. These interventions led to significant gains of knowledge $\mathrm{e}^{41,47,55,64}$, positive attitudes ${ }^{33,47}$, increase in selfassessed nutrition counselling skills ${ }^{33,55}$, and real-time practice behaviour ${ }^{33}$. The convenience, interest, and independent nature of this type of education contributed to those outcomes.

\section{Providing participants with local, practically relevant tools and messages ('Give me tools')}

Some researchers theorised that making local, practically relevant tools and messages available in practice contexts would change the behaviour of trainee healthcare professionals. The tools they provided included memorable slogans ${ }^{35}$, simple 'key take home messages ${ }^{35,39,42,63}$, personalised nutrition messages ${ }^{35}$, and locally relevant examples ${ }^{34}$. Researchers simplified nutrition messages ${ }^{35}$, provided resource materials and tools to resolve problems in counselling and assessing patients ${ }^{34,63}$, and adapted advice for local conditions ${ }^{34}$. Those interventions helped professionals engage in specific rather than generic discussions with patients, and provided advice and recommendations that patients found relevant ${ }^{34}$. The authors of a randomised controlled trial (RCT), which improved physicians' counselling of mothers with malnourished children aged 12-24 months in Bra$\mathrm{zil}^{34}$, attributed children's improved nutritional status to this provision of locally appropriate messages and tools.

\section{Using non-traditional teaching and learning strategies ('The right strategy for the right job')}

Another theory, which guided interventions, was that non-traditional teaching and learning strategies would change professionals' behaviour. For instance, Hillenbrand and Larsen ${ }^{43}$ hypothesised that providing a series of interactive educational interventions to paediatric residents would increase their knowledge about breastfeeding and lactation problems and increase their confidence to counsel breastfeeding women. Interventions which sometimes complemented lectures, included discussions, simulated patient cases, group work, role plays, hands-on demonstrations, group practice, panel discussions and case-based learning. Other interventions included problem-based learning tutorials, computer-based or web-based cases, student-led debates, self-assessment exercises, and clinical case presentations ${ }^{58,62,68}$. These interventions provided practical experience and promoted active learning. They emphasised the development of skills rather than just knowledge. They engaged participants' interest and helped them assume responsibility for their own learning. These interventions caused significant changes in participants' knowledge, personal health habits, confidence to provide exercise and dietary counselling, ability to counsel obese patients, and ability to manage malnutrition. Carson 
et al. ${ }^{68}$ attributed the enhanced nutrition counselling skills of students in a 4-week ambulatory care rotation to their innovative combination of teaching strategies.

\section{Improving self-efficacy ('I feel that I can do it, so I will do it')}

Self-efficacy is a basic tenet of Bandura's social learning theory ${ }^{80}$. This term describes individuals' confidence in their ability to perform a task or achieve an outcome. It is a key influence on behaviour ${ }^{80}$. Eight studies explicitly set out to improve participants' self-efficacy by increasing their confidence. They adopted strategies like role modelling by practising physicians ${ }^{68}$, role playing using either simulated or real patients ${ }^{43,44,48,58}$, providing demonstrations and hands-on practice sessions $35,43,44,46,48,61,68$, and viewing then discussing videos and web-based cases $^{44,68}$. Four each of these interventions were conducted among future healthcare professionals and practising healthcare professionals. They were effective in both settings.

\section{Improving the personal health habits of the healthcare professional ('Do as I do')}

Four interventions, which stimulated practising ${ }^{46,60}$ and health professions students ${ }^{58,61}$ to take better care of their own health, had positive outcomes. These included regular consumption of fruits and vegetables, personal awareness of calorie consumption, engagement in regular physical activity, and development of culinary skills. In both settings, these led to better self-reported healthy lifestyles and self-reported ability to undertake dietary assessment ${ }^{61}$, counselling confidence ${ }^{46,58,61}$, self-assessed knowledge ${ }^{60}$ and even treating a higher proportion of diabetic patients with diet alone ${ }^{60}$. Healthcare professionals, who considered themselves role models for patients, felt more confident to advise patients to do as they had done ${ }^{46,58,61}$.

\section{Initial and revised programme theory}

Our published protocol ${ }^{21}$ presented candidate theories, and a theoretical model, which we briefly repeat here. Drawing on social cognitive theory, we postulated the following:

- Healthcare professionals' ability to deliver nutrition care is influenced by their competence, which is the outcome of a learning process, which is influenced by factors within academic environments. Those factors include the quantity and quality of nutrition content in curricula, the teaching and learning methods employed, and the extent to which learning is reinforced.

- Professionals are more likely to care for patients' nutrition if they have high self-efficacy for nutrition care and vice versa.

- Professionals' delivery of nutrition care is a behaviour demonstrated in the social context of workplaces, which is influenced by observing and modelling the behaviours, attitudes and emotional reactions of others (e.g, superiors) ${ }^{81}$. It is also influenced by the structural determinants of behaviours such as the workplace settings themselves 
(e.g, hospital/community, and emergency/paediatric/general ward), job descriptions/role, time and availability of other staff to undertake particular roles.

The review process described above examined those theories, which led us to revise, add components to, and broaden our theoretical model (shown in figure 2). 'Outcomes', in realist terminology, can be short, medium and long terms ${ }^{82}$. We have added a hierarchy of outcomes to our theoretical model.

The items in the model are inter-related as opposed to operating in isolation from one another. They do not operate in a linear fashion. Several context-mechanism-outcome configurations could be generated from the data. For instance, assessment of needs identify knowledge, skills and attitude gaps and other educational needs of potential participants. The outcome of the needs assessments informs the design of the educational intervention as well as its characteristics. It informs what kind of characteristics, or strategies the intervention should adopt in order to realise the desired outcome. These strategies could include improving the personal health habits of healthcare professionals, adopting technology-based education, improving skills development, adopting innovative teaching and learning strategies, role modelling, and others. These generate mechanisms (not indicated in the diagram) such as interest, receptivity, and acceptance, which generate short-term outcomes such as improved knowledge, attitude, skills, self-efficacy, values and personal habits. The immediate and short-term outcomes may act as mechanisms to bring about change in nutrition practice behaviour (medium-term outcome). Doctors and other healthcare professionals may change their nutrition practice behaviour as a result of having adequate knowledge, skills, attitudes, confidence and self-efficacy. A change in nutrition practice behaviour will mean increased delivery of nutrition care to patients which may result in the long-term goal of improved clinical outcomes (long-term outcomes) of patients. However, these outcomes can best be enhanced and maintained if there is an enabling healthcare setting (context). This could be realised by enhancing certain conditions/contexts such as restructuring the healthcare system, removing structural and systemic barriers, adopting favourable policies for nutrition care, providing appropriate tools to deliver nutrition care, investing more in preventive care, and providing an office that makes it easier to provide nutrition care. Providing an enabling healthcare setting was central to all the $\mathrm{CMO}$ configurations identified. 


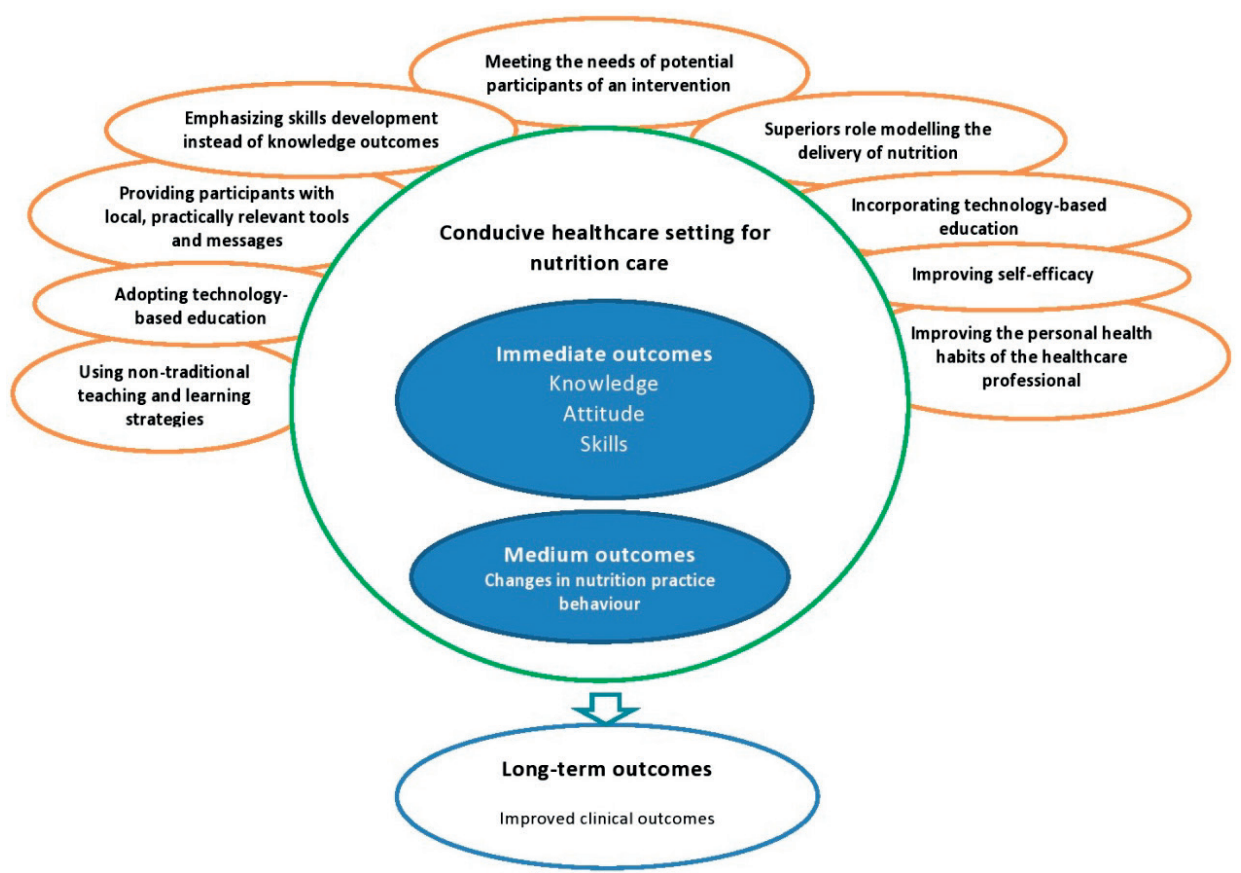

Figure 2: Revised theoretical model or programme theory

We present in Table 4 a summary of the characteristics of interventions in accordance with what works, for whom and under what conditions.

Table 4: Overview of what works, for whom, under what circumstances, to achieve what?

\begin{tabular}{ll}
\hline What works & Choosing interventions, which are educationally and clinically relevant to the needs of \\
participants & Adopting appropriate teaching and learning techniques \\
& Building on self-efficacy and confidence through role modelling \\
& Emphasizing skills development rather than pure knowledge gains \\
Improving the personal lifestyle habits of healthcare professionals & Removing systemic barriers and restructuring healthcare systems to make healthcare \\
& settings more enabling \\
& Using practical, relevant tools \\
& Using Information and Communications Technology (computer-based education) \\
& Doctors and other practising healthcare professionals \\
For whom & Students of the health professions \\
& Within a multidisciplinary approach to nutrition education and care \\
Under what & Supported by both educational and care delivery leaders \\
circumstances & Where nutrition care is recognized as an important component of: \\
& Care delivery systems \\
& Curricula \\
& Where healthcare systems are structured to be conducive to the practice of nutrition \\
care &
\end{tabular}




\section{Measurement issues}

The ultimate aim of health education is to improve health outcomes. Few studies have, however, even tried to show improvements in patients' health because it is very difficult to do. Authors acknowledged that this limited the conclusions they could draw from their evidence ${ }^{62}$ ), which meant that they could often only speculate on how their interventions might affect patients' health. The impact of educational interventions is often ranked according to its position in Miller's pyramid of assessment ${ }^{83}$. Some studies achieved the highest level - the performance level - which is most likely to impact patient outcomes. They did so by directly observing the delivery of nutrition care in clinical settings ${ }^{14,34,43,45,50}$, recording videos of doctors counselling patients ${ }^{14}$, auditing charts $^{42,65,84}$, and using incognito simulated patients ${ }^{33}$. Most studies were at lower levels of the pyramid. For example, they assessed participants' reported changes in practice behaviours by means of self-administered surveys. As observed by the authors of one such study ${ }^{58}$, reliance on students' self-reported confidence in counselling rather than an objective measure of counselling skills (such as an objective-structured clinical examination) limits the generalisability of the findings. Schlair et al ${ }^{61}$ acknowledged the potential for social desirability bias in self-reports. While self-report would be invalid evidence in a systematic review or meta-analysis, it is safer to use it in realist synthesis, which aims to produce progressively more refined theories of change rather than incontrovertible evidence.

For future studies, Scolapio et al ${ }^{62}$ suggested that 'harder' data could be obtained using patient surveys and chart reviews, or having participants give specific examples of improved patient outcome that were directly linked to competences they had acquired from educational interventions. Our review shows the need for future studies to explore innovative ways of collecting this information ${ }^{62}$.

\section{Discussion}

There is increasing pressure for medical education to be socially accountable $\mathrm{e}^{85-87}$. This research may be seen as socially responsive because it arose out of a pressing health need in sub-Saharan Africa: improvement of the competencies of doctors and other healthcare professionals in order to deliver effective nutrition care. Our study provided practical guidance to educators trying to meet this need in Africa and elsewhere by showing the importance of moving education for nutrition care beyond the simple acquisition of knowledge.

The CMO configurations identified in this realist review are preliminary and non-exhaustive and should be considered as a set of generic hypotheses derived from the best available evidence. Nonetheless, they provide information to policymakers about what may improve the nutrition practice behaviour of healthcare professionals, how, under 
what conditions, and in what settings. Our review has identified a set of conditions that facilitate the success of interventions in varied contexts.

A key finding of this review is that improving the skills, self-efficacy, and attitudes of learners by adopting appropriate teaching and learning strategies is critical to the success of nutrition education interventions. Improving learners' skills and attitudes provides them with confidence and a sense of enactive mastery of the specific tasks they have to perform. Role modelling of the delivery of nutrition care by superiors, providing appropriate physical space in which to deliver nutrition care, and adopting favourable policies are important because they increase professionals' sense of being accepted, credibility, relatedness and assurance.

Our analysis shows that planners of educational interventions would be well advised to assess potential participants' needs and interests. Computer-based education presents new opportunities for course designers and planners. Already considered as a potentially efficient form of teaching and learning in the health professions ${ }^{47,88-90}$, this presents novel ways of incorporating nutrition content into healthcare professional curricula. Given that healthcare professionals say they have too little time to attend training programmes and provide nutrition care, the convenience of computer-based and internetbased education has potential to overcome barriers to learning.

The main strengths of our review were its integrative nature and our use of realist synthesis methodology, which allowed for practical theories to be generated for future testing and implementation. However, the review had limitations. One is that we did not consult individual experts in the field when we developed our initial model. Had we done that, we might have included more candidate theories. We also acknowledge the interpretive and subjective nature of qualitative research and the likelihood that a different team of researchers might have arrived at different candidate programme theories. We acknowledge that the model shown in figure 2 is but one of several possible interpretations, as is typical of the models that emerge from realist synthesis. We acknowledge limitations in the evidence base. The synthesis that results from any review is only as good as the primary studies it is able to include. Many of the primary studies provided limited, superficial descriptions of their educational interventions. This made it difficult for us to test all components of our candidate theories and to provide rich descriptions of some of the mechanisms that were identified. As has been found by other reviewers in medical education $22,29,30$, this review was limited by a lack of descriptions of the contexts of the intervention, implementation processes, and mechanisms.

Other limitations included the unavailability of the full text of seven studies ${ }^{91-96}$. Whilst it is a limitation, realist synthesis is less dependent on the inclusion of complete sets of studies than, for example, traditional systematic reviews ${ }^{82}$. So, it may limit the scope of our findings but does not invalidate them. While the backbone of meta-analysis and traditional systematic review is aggregation, realist synthesis refines theories by obtaining a rich (rather than necessarily complete) evidence base of reports of how interventions generate certain pattern of outcome ${ }^{82}$. We also consider as a limitation the 
delay in preparing the review for publication after the end of the search in December 2014. During this period new studies might have been published, the inclusion of which may enrich further our findings. Finally, initial screening by just one author might be seen as a limitation but we found such high consistency between that author's judgement and a second author in a pilot phase of the project that we judged single screening to make the best use of the inevitably limited resources in the country, where the research was conducted.

We conclude that it has been possible to assemble, from a heterogeneous database, some patterns in the links between CMOs that are consistent enough to guide the practice of nutrition education. Our findings have refined some existing candidate theories, which researchers, also, apply to their work on nutrition education.

\section{Author affiliations}

${ }^{1}$ Department of Health Professions Education and Innovative Learning, School of Medicine and Health Sciences, School of Medicine and Health Sciences, University for Development Studies, Tamale, Ghana

${ }^{2}$ Department of Medicine and Life Sciences, School of Health Professions Education, Faculty of Health, Maastricht University, Maastricht, The Netherlands

${ }^{3}$ Department of Community Nutrition, School of Allied Health Sciences, University for Development Studies, Tamale, Ghana

${ }^{4}$ Department of Health Services Research, Institute of Psychology, Health and Society, University of Liverpool, Liverpool, UK

Acknowledgements: The authors wish to thank Mr. Fuseini Abdulai Braimah, senior library assistant, School of Medicine and Health Sciences, University for Development Studies, Ghana for his support in helping to retrieve full texts of some of the included studies.

Contributors: VM and TD were responsible for the conception and design of the study. VM was principally responsible for searching, sourcing and initial screening of studies. VM, MGC and TD further screened studies based on abstracts. Quality assessment was performed by VM, AJJAS, FS and MGC. VM was responsible for data extraction, synthesis and interpretation of the data and drafting of the manuscript. AJJAS, TD, FS, MGC and PA contributed to the interpretation of the data and revision of the article. All authors approved the article for publication.

Funding: This research received no specific grant from any funding agency in the public, commercial or not-for-profit sectors.

Competing interests: None declared

Provenance and peer review: Not commissioned; externally peer reviewed. 
Data sharing statement: VM had full access to all the data in the study, and takes responsibility for the integrity of the data and the accuracy of the data analysis. 


\section{References}

1. Pearson TA, Blair SN, Daniels SR, et al. AHA guidelines for primary prevention of cardiovascular disease and stroke: 2002 update consensus panel guide to comprehensive risk reduction for adult patients without coronary or other atherosclerotic vascular diseases. Circulation 2002;106(3):388-391.

2. Bantle JP, Wylie-Rosett J, Albright AL, et al. Nutrition recommendations and interventions for diabetes: a position statement of the American Diabetes Association. Diabetes Care 2008;31:S61-S78.

3. Goldstein LB, Adams R, Alberts MJ, et al. Primary Prevention of Ischemic Stroke A Guideline From the American Heart Association/American Stroke Association Stroke Council: Cosponsored by the Atherosclerotic Peripheral Vascular Disease Interdisciplinary Working Group; Cardiovascular Nursing Council; Clinical Cardiology Council; Nutrition, Physical Activity, and Metabolism Council; and the Quality of Care and Outcomes Research Interdisciplinary Working Group: The American Academy of Neurology affirms the value of this guideline. Stroke 2006;37(6):1583-1633.

4. United Nations Children's Fund. Improving Child Nutrition:The achievable imperative for global progress. New York: United Nations Children's Fund, 2013:1-124

5. Kopelman P, Lennard-Jones J. Nutrition and patients: a doctor's responsibility. Clin Med 2002;2(5):391394.

6. General Medical Council. Tomorrow's doctors: Outcomes and standards for undergraduate medical education. Manchester, UK: General Medical Council. 2009.

7. Abid A, Galuska D, Khan L, Gillespie C, Ford E, Serdula M. Are healthcare professionals advising obese patients to lose weight? A trend analysis. MedGenMed: Meds Gen Med 2004;7(4):10-10.

8. Tsai AG, Wadden TA. Treatment of obesity in primary care practice in the United States: a systematic review. J Gen Intern Med 2009;24(9):1073-1079.

9. Abramson S, Stein J, Schaufele M, Frates E, Rogan S. Personal exercise habits and counseling practices of primary care physicians: a national survey. Clin J Sport Med 2000;10(1):40-48.

10. Huang J, Yu H, Marin E, Brock S, Carden D, Davis T. Physicians' weight loss counseling in two public hospital primary care clinics. Acad Med 2004;79(2):156-161.

11. Laschinger HKS, Tresolini CP. An exploratory study of nursing and medical students health promotion counselling self-efficacy. Nurse Educ Today 1999;19(5):408-418.

12. Jay M, Gillespie C, Ark T, et al. Do internists, pediatricians, and psychiatrists feel competent in obesity care? J Gen Intern Med 2008;23(7):1066-1070.

13. Foster GD, Wadden TA, Makris AP, et al. Primary care physicians' attitudes about obesity and its treatment. Obes Res 2003;11(10):1168-1177.

14. Tziraki C, Graubard BI, Manley M, Kosary C, Moler JE, Edwards BK. Effect of Training on Adoption of Cancer Prevention Nutrition-Related Activities by Primary Care Practices: Results of a Randomized, Controlled Study. J Gen Inten Med 2000;15(3):155-162.

15. McLaren DS. Nutrition in medical schools: a case of mistaken identity. Am J Clin Nutr 1994;59(5):960-963.

16. Weinsier RL. National Dairy Council Award for Excellence in Medical/Dental Nutrition Education Lecture, 1995: medical-nutrition education--factors important for developing a successful programme. Am J Clin Nutr 1995;62(4):837-840.

17. Gershoff SN. National Dairy Council Award for Excellence in Medical/Dental Nutrition Education Lecture, 1996: nutrition education--success or failure? Am J Clin Nutr 1996;64(5):809-812.

18. Ball EL, Hughes MR, Leveritt DM. Nutrition in general practice: role and workforce preparation expectations of medical educators. Austr J Prim Health 2010;16(4):304-310.

19. Mechanic D. Social research in health and the American sociopolitical context: the changing fortunes of medical sociology. Soc Sci Med 1993;36(2):95-102.

20. Sunguya BF, Poudel KC, Mlunde LB, Urassa DP, Yasuoka J, Jimba M. Nutrition training improves health workers' nutrition knowledge and competence to manage child undernutrition: a systematic review. Frontiers in Public Health. 2013;1:37. 
21. Mogre V, Scherpbier A, Dornan T, Stevens F, Aryee PA, Cherry MG. A realist review of educational interventions to improve the delivery of nutrition care by doctors and future doctors. Syst Rev. 2014;3(1):148.

22. Wong G, Greenhalgh T, Pawson R. Internet-based medical education: a realist review of what works, for whom and in what circumstances. BMC Med Educ 2010;10(1):12.

23. Pawson R, Greenhalgh T, Harvey G, Walshe K. Realist synthesis: An introduction: ESRC Research methods programmeme, University of Manchester RMP: Methods Paper 2. 2004. http://www.ccsr.ac.uk/methods/publications/documents/RMPmethods2.pdf

24. McCormack B, Rycroft-Malone J, DeCorby K, et al. A realist review of interventions and strategies to promote evidence-informed healthcare: a focus on change agency. Implement Sci 2013;8(1):107.

25. Pawson R. Evidence-based policy: A realist perspective. Thousand Oaks (CA): Sage, 2006.

26. Pawson R, Greenhalgh T, Harvey G, Walshe K. Realist review-a new method of systematic review designed for complex policy interventions. Journal of health services research \& policy. 2005;10(suppl 1):21-34.

27. Westhorp G, Walker B, Rogers P, Overbeeke N, Ball D, Brice G. Enhancing community accountability, empowerment and education outcomes in low and middle-income countries: A realist review. EPPIcentre, social science research unit, institute of education. London: University of London. 2014.

28. Pluye P, Robert E, Cargo M, et al. Proposal: A mixed methods appraisal tool for systematic mixed studies reviews. Montréal: McGill University. 2011.

29. Yardley S, Cottrell E, Rees E, Protheroe J. Modelling successful primary care for multimorbidity: a realist synthesis of successes and failures in concurrent learning and healthcare delivery. BMC Fam Pract 2015;16(1):23.

30. Jones AC, Shipman SA, Ogrinc G. Key characteristics of successful quality improvement curricula in physician education: a realist review. BMJ Qual Saf 2015;24(1):77-88.

31. Steinert Y, Mann K, Centeno A, et al. A systematic review of faculty development initiatives designed to improve teaching effectiveness in medical education: BEME Guide No. 8. Med Teach 2006;28(6):497-526.

32. Shamseer $L$, Moher $D$, Clarke $M$, et al. Preferred reporting items for systematic review and meta-analysis protocols (PRISMA-P) 2015: elaboration and explanation. BMJ 2015;349:g7647.

33. Maiburg BHJ, Rethans JE, Schuwirth LWT, Mathus-Vliegen LMH, van Ree JW. Controlled trial of effect of computer-based nutrition course on knowledge and practice of general practitioner trainees. Am J Clin Nutr 2003;77(4S):1019S-1024.

34. Pelto GH, Santos I, Gonçalves H, Victora C, Martines J, Habicht J-P. Nutrition counseling training changes physician behavior and improves caregiver knowledge acquisition. J Nutr 2004;134(2):357-362.

35. Levy J, Harris J, Darby P, Sacks R, Dumanovsky T, Silver L. The primary care nutrition training programme: An approach to communication on behavior change. Health Promot Pract 2011;12(5):761-768.

36. Carson JAS. Pocket tape measure for waist circumference: Training medical students and residents on a simple assessment of body composition. Journal of Nutrition. 2003;133(2):547S-549S.

37. Taren DL, Thomson CA, Koff NA, et al. Effect of an integrated nutrition curriculum on medical education, student clinical performance, and student perception of medical-nutrition training. American Journal of Clinical Nutrition. 2001;73(6):1107-1112.

38. Buckley KM. Evaluation of classroom-based, Web-enhanced, and Web-based distance learning nutrition courses for undergraduate nursing. J Nurs Educ 2003;42(8):367-370.

39. Ray S, Udumyan R, Rajput-Ray M, et al. Evaluation of a novel nutrition education intervention for medical students from across England. BMJ Open 2012;2(1): e000417.

40. Ke L-S, Chiu T-Y, Hu W-Y, Lo S-S. Effects of educational intervention on nurses' knowledge, attitudes, and behavioral intentions toward supplying artificial nutrition and hydration to terminal cancer patients. Support Care Cancer 2008;16(11):1265-1272.

41. Buchowski MS, Plaisted C, Fort J, Zeisel SH. Computer-assisted teaching of nutritional anemias and diabetes to first-year medical students. Am J Clin Nutr 2002;75(1):154-161.

42. Puoane T, Sanders D, Ashworth A, Ngumbela M. Training nurses to save lives of malnourished children. Curationis 2006;29(1):73-78. 
43. Hillenbrand KM, Larsen PG. Effect of an educational intervention about breastfeeding on the knowledge, confidence, and behaviors of pediatric resident physicians. Pediatrics 2002;110(5).

44. Ockene JK, Ockene IS, Quirk ME, et al. Physician training for patient-centered nutrition counseling in a lipid intervention trial. Prev Med 1995;24(6):563-570.

45. Zaman S, Ashraf RN, Martines J. Training in complementary feeding counselling of healthcare workers and its influence on maternal behaviours and child growth: a cluster-randomized controlled trial in Lahore, Pakistan. J Health Popul Nutr 2008;26(2):210.

46. Eisenberg DM, Miller AM, McManus K, Burgess J, Bernstein AM. Enhancing medical education to address obesity: "See one. Taste one. Cook one. Teach one.". JAMA Intern Med 2013;173(6):470-472.

47. Roche PL, Ciccarelli MR, Gupta SK, Hayes BM, Molleston JP. Multi-School Collaboration to Develop and Test Nutrition Computer Modules for Pediatric Residents. J Am Diet Assoc 2007;107(9):1586-1589.

48. Gance-Cleveland B, Sidora-Arcoleo K, Keesing H, Gottesman MM, Brady M. Changes in nurse practitioners' knowledge and behaviors following brief training on the healthy eating and activity together (HEAT) guidelines. J Pediatr Health Care 2009;23(4):222-230.

49. Ray $S$, Laur $C$, Douglas $P$, et al. Nutrition education and leadership for improved clinical outcomes: training and supporting junior doctors to run 'Nutrition Awareness Weeks' in three NHS hospitals across England. BMC Med Educ 2014;14(1):109.

50. Bassichetto KC, Réa MF. Infant and young child feeding counseling: an intervention study. J Pediatr (Rio J) 2008;84(1):75-82.

51. Dacey M, Arnstein F, Kennedy MA, Wolfe J, Phillips EM. The impact of lifestyle medicine continuing education on provider knowledge, attitudes, and counseling behaviors. Med Teach 2013;35(5):e1149e1156.

52. Ritenbaugh CK, Thomson CA, Taren D, et al. Nutrition curriculum in medical education: An integrated and comprehensive approach. Teach Learn Med 1996;8(2):102-110.

53. Edwards L, Wyles D. The folic acid message-can training make a difference? Hum Nutr Diet 1999;12(4):317-326.

54. Castro MG, Pompilio CE, Horie LM, Verotti CCG, Waitzberg DL. Education programme on medical nutrition and length of stay of critically ill patients. Clin Nutr 2013;32(6):1061-1066.

55. Kohlmeier M, Althouse L, Stritter F, Zeisel SH. Introducing cancer nutrition to medical students: effectiveness of computer-based instruction. Am J Clin Nutr 2000;71(4):873-877.

56. Bjerrum M, Tewes M, Pedersen P. Nurses' self-reported knowledge about and attitude to nutrition-before and after a training programmeme. Scand J Caring Sci 2012;26(1):81-89.

57. Pedersen PU, Tewes M, Bjerrum M. Implementing nutritional guidelines-the effect of systematic training for nurse nutrition practitioners. Scand J Caring Sci 2012;26(1):178-185.

58. Conroy MB, Delichatsios HK, Hafler JP, Rigotti NA. Impact of a preventive medicine and nutrition curriculum for medical students. American Journal of Prev Med 2004;27(1):77-80.

59. Endevelt R, Shahar DR, Henkin Y. Development and implementation of a nutrition education programme for medical students: A new challenge. Educ Health (Abingdon) 2006;19(3):321-330.

60. de Fine Olivarius N, Palmvig B, Andreasen AH, Thorgersen JT, Hundrup C. An educational model for improving diet counselling in primary care A case study of the creative use of doctors' own diet, their attitudes to it and to nutritional counselling of their patients with diabetes. Patient Educ Couns 2005;58(2):199-202.

61. Schlair S, Hanley K, Gillespie C, et al. How medical students' behaviors and attitudes affect the impact of a brief curriculum on nutrition counseling. J Nutr Educ Behav 2012;44(6):653-657.

62. Scolapio JS, Dibaise JK, Schwenk WF, 2nd, Macke ME, Burdette R. Advances and controversies in clinical nutrition: the education outcome of a live continuing medical education course. Nutr Clin Pract 2008;23(1):90-95.

63. Kennelly S, Kennedy N, Rughoobur G, Slattery CG, Sugrue S. An evaluation of a community dietetics intervention on the management of malnutrition for healthcare professionals. I Hum Nutr Diet 2010;23(6):567-574. 
64. Lewis KO, Frank GR, Nagel R, et al. Pediatric trainees' engagement in the online nutrition curriculum: preliminary results. BMC Medi Educ 2014;14(1):190.

65. Acuna K, Pires C, Santos G, et al. Detection of nosocomial malnutrition is improved in Amazon region by a standard clinical nutrition education programme. Nutr Hosp 2008;23(1):60-67.

66. Powell-Tuck J, Summerbell C, Holsgrove G, Garrow J. Four years' experience of an undergraduate medical nutrition course. J R Soc Med 1997;90(2):67-72.

67. Afaghi A, Mohamadi AAHA, Ziaee A, Sarchami R. Effect of an Integrated Case-based Nutrition Curriculum on Medical Education at Qazvin University of Medical Sciences, Iran. Glob J Health Sci 2012;4(1):112.

68. Carson JAS, Gillham MB, Kirk LM, Reddy ST, Battles JB. Enhancing self-efficacy and patient care with cardiovascular nutrition education. Am J Prev Med 2002;23(4):296-302.

69. Vanderpool C, Lin HC, Scheimann A. NASPGHAN Nutrition University as a Model for Continuing Education Within Pediatric Nutrition. J Pediatr Gastroenterol Nutr 2014;58(4):469-471.

70. Duerksen DR. Teaching medical students the subjective global assessment. Nutrition. 2002;18(4):313-315.

71. Engel SS, Crandall J, Basch CE, Zybert P, Wylie-Rosett J. Computer-assisted diabetes nutrition education increases knowledge and self-efficacy of medical students. Diabetes Educ 1997;23(5):545-549.

72. Richards D, Mitchell G. Insight. GPs and nutrition: what they want and how they want it. A GP nutrition education project. Aust J Nutr Diet 2001;58(1):56-59.

73. Kipp DE. Technological methods in nutrition education. Developing interactive computerized modules accessible on the World Wide Web for medical students. Top Clin Nutr 1997;12(2):38-44.

74. Cooksey K, Kohlmeier M, Plaisted C, Adams K, Zeisel SH. Getting nutrition education into medical schools: a computer-based approach. Am J Clin Nutr 2000;72(3):868S-876S.

75. Cheatham M, Boosalis MG, Boissonneault GA. Use of a Computer Tutorial on Nutritional Assessment by Three Different Groups of Health Professions Students. J Allied Health 2002;31(4):252-255.

76. Kolasa KM, Elesha-Adams M, Patton DD. When a reporter calls: media training to teach nutrition education. Top Clin Nutr 1996;11(4):75-80.

77. Fox AL. Evaluation of a Pilot Arts and Health Module In a Graduate Community Nutrition Programme. Can J Diet Pract Res 2009;70(2):81-86.

78. Ockene JK, Kristeller J, Goldberg R, et al. Increasing the efficacy of physician-delivered smoking interventions. J Gen Intern Med 1991;6(1):1-8.

79. Bandura A, McClelland DC. Social learning theory. Englewood Cliffs (NJ): Prentice-Hall, 1977.

80. Bandura A. Self-efficacy: The exercise of control: Macmillan, 1997.

81. Bandura A. Social cognitive theory: An agentic perspective. Annu Rev Psychol 2001;52(1):1-26.

82. Westhorp G, Walker B, Rogers P. Under what circumstances does enhancing community accountability and empowerment improve education outcomes, particularly for the poor? A realist synthesis protocol. London: EPPI-Centre, Social Science Research Unit, Institute of Education, University of London, 2012

83. Miller GE. The assessment of clinical skills/competence/performance. Acad Med 1990;65(9):S63-67.

84. Lindgren S, Karle H. Social accountability of medical education: aspects on global accreditation. Med Teach 2011;33(8):667-672.

85. Boelen C, Woollard R. Social accountability: The extra leap to excellence for educational institutions. Med Teach 2011;33(8):614-619.

86. Boelen C, Woollard B. Social accountability and accreditation: a new frontier for educational institutions. Med Educ 2009;43(9):887-894.

87. Devitt P, Palmer E. Computer-aided learning: an overvalued educational resource? Med Educ 1999;33(2):136-139.

88. Carr MM, Reznick RK, Brown DH. Comparison of computer-assisted instruction and seminar instruction to acquire psychomotor and cognitive knowledge of epistaxis management. Otolaryngol Head Neck Surg 1999;121(4):430-434.

89. D'Alessandro DM, Kreiter CD, Erkonen WE, Winter RJ, Knapp HR. Longitudinal follow-up comparison of educational interventions: multimedia textbook, traditional lecture, and printed textbook. Acad Radiol 1997;4(11):719-723. 
90. Deen D, Karp R, Lowell B. A mini-fellowship in clinical nutrition for primary care physicians. J Cancer Educ 2000;15(3):134-136.

91. Heimburger DC, Ullmann DO, Ramsey MJ, et al. Dietary habits of first-year medical students assessed during clinical nutrition course. Nutr 1994;10(3):214-220.

92. Hodgson CS, Wilkerson L, Go VLW. Changes in nutrition knowledge among first- and second-year medical students following implementation of an integrated nutrition curriculum. J Cancer Educ 2000;15(3):144147.

93. Kolasa KM, Jobe AC, Miller MG, Clay MC. Teaching medical students cancer risk reduction nutrition counseling using a multimedia programme. Fam Med 1999;31:200-204.

94. Edwards M. Nutrition education for medical students: 4th year transition to residency for primary care (1020.3). The FASEB Journal. 2014;28(1 Supplement):1020.1023.

95. Carson JAS. Impact of integrating cardiovascular nutrition in an ambulatory care rotation on the knowledge, attitudes, self-efficacy and patient care practices of fourth year medical students. Doctoral dissertation. University of Texas-Austin, 2000.

96. Baker K, Olfert M. Incorporating nutrition education through applied, hands-on culinary elective in medical school training (1020.2). The FASEB Journal. 2014;28(1 Supplement):1020.1022. 
Chapter

General Discussion 



\section{Introduction}

In this Chapter, we provide a general discussion of the research questions outlined in Chapter 1 in accordance with the findings of the studies presented in this thesis. Conclusions are drawn, and the strengths of the studies are highlighted. Furthermore, ideas for future research are also presented. Lastly, we describe the limitations of the studies of this thesis and the implications of the evidence for medical education and clinical practice.

\section{Research question 1}

How do medical students perceive the role of nutrition care in their training, and what do they perceive to be the roles and responsibilities of doctors regarding nutrition care? (Chapters $2 \& 6$ )

Answers to this research question have been provided by the results of chapters 2 and 6 . Based on the observation that nutrition care and education are not core elements of medical education, we aimed to explore medical students or 'soon to be doctors' perception of the role and importance of nutrition care and education in patient care as well as in their training. Furthermore, we explored the acceptability and recognition of their roles and responsibility as medical students in the provision of nutrition care as of now and after graduation.

The findings of Chapter 2 show that medical students recognise that nutrition is important to patient care, and they also note that nutrition education is and should be an important aspect of their training in medical school. They identified that as medical doctors they will have a special position to efficiently provide nutrition care to help patients to adopt healthy nutrition and dietary habits. As identified by them, some of these opportunities are: doctors are the first point of call when patients seek care; doctors have regular contact with patients; patients have trust in doctors' advice and the provision of nutrition care ensures a holistic approach to healthcare. It is important to also note that these perspectives of medical students are in line with previous reports conducted among practicing physicians ${ }^{1,2}$.

Furthermore, results of Chapter 2 report that medical students recognise that every healthcare professional has an important role to play in the provision of nutrition care and that doctors should be more concerned about their patients' nutrition care.

Our findings demonstrate students' positive attitude towards nutrition care. If maintained until they will practice, this attitude may result in improved nutrition practice and patient care. This is in line with previous studies that have shown associations between nutrition practice behaviour, attitudes towards nutrition care ${ }^{3}$ and patients' preference of type of healthcare professional to receive nutrition care ${ }^{2,4-7}$. 
Our finding in Chapter 2 that medical students notice that patients consider them to be important and credible sources of nutrition information and nutrition care providers demonstrates that these medical students are aware of the expectations of patients regarding nutrition care.

Consistent with previous studies ${ }^{1,8}$ doctors' role in nutrition care as identified by medical students align with the nutrition care cycle. Some of these were: provision of nutrition advice; nutrition diagnosis; and monitoring patient's progress in nutrition care. Being the first line of contact in the healthcare system, medical students opined in Chapter 6 that doctors are in an important position of identifying patients who may need nutrition care, providing those patients with nutrition care and, subsequently, referring them to dieticians/nutritionists for specialist dietary care.

Given that doctors drive the healthcare system in Ghana, coupled with patients' trust in them, they could be effective in advocating nutrition care for patients and reinforcing nutrition care provided by nutritionists/dieticians, which may result in improved clinical outcomes (Chapter 6).

\section{Conclusions regarding research question 1}

Medical students underscored the important role of the doctor in helping patients to adopt healthy dietary habits and also recognise the need to learn about nutrition during medical school. They reiterated the fact that doctors, as gatekeepers and care coordinators, are in a good position to provide nutrition care.

\section{Directions for future research}

1. It is yet to be known whether medical students' positive perceptions and acceptability regarding nutrition care and education will translate into improved nutrition practice behaviour. Future research should thus consider whether having positive attitudes and recognition of the need for nutrition education will result in improved nutrition care competencies and practice behaviour.

2. To promote inter-professional healthcare delivery, future studies should explore the perceptions and views of nutritionists/dieticians regarding the role of doctors in the provision of care. Answers should be found for questions such as: Do nutritionist/dieticians recognise that doctors have an important role to play in the provision of nutrition care? Do they accept that doctors should be providing some form of nutrition care? How will doctors support them in the provision of nutrition care?

3. Future studies should explore patients' perspectives and expectations concerning nutrition counselling from doctors. Do they expect to receive nutrition counselling and advice from their medical doctors?

4. Evaluations should be done to investigate the influence of doctors' provision of nutrition care on patient's adoption of healthy dietary and nutrition habits. 


\section{Research question 2}

What is the nutrition care competence (i.e., knowledge, skills and attitudes) and self-efficacy of medical students and practicing doctors? (Chapters 3, 4, 5 \& 6)

Competence is a dynamic concept that encompasses the knowledge, skills and attitudes that facilitate safe and effective health care practices ${ }^{9}$. Nutrition care competence may include knowledge about nutrition, attitudes that are conducive towards providing nutrition care and skills in providing nutrition care ${ }^{10}$. Nutrition care competency is thus necessary to support doctors to provide safe and effective nutrition care to patients. Selfefficacy in nutrition care refers to having the confidence to provide safe and effective nutrition care to patients. Just like competence, self-efficacy is also a necessary step to the provision of nutrition care. To support best practice delivery of health care, it is essential to evaluate the nutrition care competence and self-efficacy of (future) doctors in order to support them to provide safe and effective nutrition care.

Two groups of participants can be identified: medical students and practicing doctors. Future medical doctors are a group to evaluate nutrition care competence in order to determine their readiness and preparedness to incorporate nutrition care into practice and also to help determine if additional nutrition education will be needed during medical school. Assessing practicing doctors' nutrition care competence will help to estimate the adequacy of their nutrition education during medical school and to identify the need for continuing education in nutrition.

Thus, in chapters $3,4,5$ \& 6 we described nutrition care competence, self-efficacy, preparedness to provide nutrition care and adequacy of nutrition care among medical students and practicing doctors.

Although medical students and practicing doctors alike perceived nutrition care and education relevant to their training and practice, we found them reportedly feeling inadequately prepared and dissatisfied with the quality and quantity of their nutrition education (chapters 3, 4, 5 \& 6). In line with previous studies ${ }^{11-13}$, both practicing doctors and medical students conclude that their nutrition education was/is inadequate and thus feel inadequately prepared to provide nutrition care. Both medical students and practicing doctors are aware and conscious of their inadequacy regarding nutrition education and practice.

We reported in chapters 3 and 5 that perception of inadequate nutrition education is associated with students' reports of feeling inadequately prepared to provide nutrition care. Obviously, if students receive adequate nutrition education, they are more likely to feel competent and prepared to provide nutrition care. This assertion was confirmed by the results of chapter 5, where we found that perceived adequacy and satisfaction with nutrition education were associated with doctors feeling more comfortable discussing 
nutrition topics with their patients. Those who felt their nutrition education to be inadequate spent less time on nutrition counselling to their patients. This brings to light the need to improve nutrition education in medical curricula.

Similar to reports from elsewhere, Ghanaian medical students ${ }^{14-16}$ and practicing doctors $8,11,17,18$ alike had positive attitudes towards nutrition care (chapter 4). However, medical students had important knowledge gaps, and both groups felt inadequate and less self-efficacious. Among the nutrition-related knowledge domains that were assessed, these medical students' nutrition-related knowledge regarding malnutrition in children was insufficient. We find this worrying because malnutrition in children is endemic in most parts of Ghana, especially within the Northern region and the two Upper Regions of Ghana, the catchment area of the UDS-SMHS.

Again, in chapters 4 and 5, both medical students and doctors reported low self-efficacy in nutrition care. While medical students felt less efficacious and unprepared to provide nutrition care, practicing doctors felt less comfortable and confident providing nutrition counselling to their patients. Although these findings are similar to those from previous reports $8,11,17,18$, it gives an idea of the quality of nutrition education received by medical doctors in Ghana.

Some other important associations were also presented in chapters 4 and 5. Possessing a positive attitude is very important in one's perception of feeling comfortable/confident or self-efficacious to provide nutrition care. We found among both medical students and practicing doctors that positive attitude towards nutrition care was associated with self-efficacy in nutrition care.

In chapter 4, we found that students' level of knowledge in basic nutrition concepts differed by level of clinical training. Those in clinical year two and three scored lower than their counterparts in clinical year one. This suggests that students in the higher levels of clinical training might have become unfamiliar with the basic nutrition concepts they learned during the early part of their training. The findings also suggest that early incorporation of nutrition education into the medical curriculum without reinforcement in later years may not result in improved nutrition care competencies. These findings are important for curriculum planners and researchers of health professions education to design appropriate educational interventions to improve nutrition care competence and practice.

\section{Conclusions regarding research question 2}

Both medical students and practicing doctors felt unsatisfied with the quality and quantity of their nutrition education, and they considered it inadequate. Medical students felt unprepared to provide nutrition care and may be reluctant to provide such care to patients. Medical students had insufficient knowledge regarding nutrition topics that are of relevance to the growth and development of children. Both medical students and practicing doctors had positive attitudes towards nutrition care and education and considered 
these relevant to their practice; however, they felt less self-efficacious and unconfident to provide nutrition care. Perceived adequacy and satisfaction with nutrition education are important determinants of self-efficacy in nutrition care. Given that the survey we did among doctors, reported in Chapter 5, included practicing doctors who graduated from almost all of the medical schools in Ghana - it is only safe for us to conclude that nutrition education is inadequate in medical education in Ghana.

\section{Directions for future research}

1. This is the first study to investigate nutrition education in the medical curriculum in Ghana. It is important that more studies will be done in other medical schools to substantiate our findings. In this way, the inadequacy of nutrition education in the country will become more recognizable, helping persuade stakeholders to respond appropriately.

2. Little is still known about the effects of students' attitudes towards nutrition care and subsequent nutrition practice behaviour after graduation. Future studies are needed to substantiate current findings.

3. Further evidence is needed to elucidate the effects of nutrition care competence on nutrition practice behaviour of doctors, patient's adoption of healthy dietary habits and subsequent improvement in clinical outcomes.

\section{Research question 3}

What are practicing doctors' nutrition care practices and referral behaviours in the general practice setting, and what are the barriers to the provision of nutrition care? (Chapters $5 \& 6$ )

This research question focuses on the perspectives of doctors regarding their nutrition practice behaviour and referral of patients to dieticians for specialist dietary care. Furthermore, doctors' perception of the extent to which barriers negatively impact their ability to provide the needed nutrition care to their patients in the general practice setting is investigated. The results of chapters 5 and 6 provide answers to this research question.

As expected, we found that a majority of practicing doctors (76\%) recognised that over $60 \%$ of their patients will benefit from nutrition care. However, they were able to provide such nutrition care to fewer than $60 \%$ of patients in need. This was aggravated by the majority of doctors providing nutrition counselling per patient for less than 6 minutes. Thus, the provisions of nutrition care by these doctors fell below their own expectations and likely that of patients. This finding is consistent with previous findings that the nutrition practice behaviour of doctors is poor ${ }^{11,17}$. Doctors' poor nutrition practice 
behaviour in the general practice setting has been the impetus for researchers in health professions education and international bodies' calls for improvement of nutrition education in the medical curriculum ${ }^{19,20}$. The calls for improvement in medical nutrition education probably stems from the finding reported in chapter 5 and previous studies ${ }^{11,21}$ that the majority of practicing doctors identify inadequate competence and self-efficacy in nutrition care as well as poor nutrition counselling as barriers that hinder their ability to provide nutrition care to their patients as expected. Our findings add to these calls.

It is important to note that doctors' competence in nutrition care and self-efficacy is informed by the quality and quantity of nutrition education they received during medical school. The kind of care that can be provided by a doctor to a patient is largely influenced by the training received. The results of chapter 5 and 6 and the answers to research question 2 confirm this assertion.

Demonstrating the influence of quality and quantity of nutrition educational experiences on nutrition practice behaviour, we reported in chapter 5 that practicing doctors who perceived their nutrition educational experiences during medical school to be inadequate were significantly ( $p=0.009$ ) less likely to spend time discussing nutrition topics with their patients.

As presented in chapter 5, the majority of doctors (85\%) referred patients to dieticians/nutritionists for specialist care. Practicing doctors recognised the need for interprofessional collaboration as well as their limitations regarding nutrition care. We however found it worrying that doctors' frequency of referring patients to dieticians/nutritionist did not correspond with the proportion of patients they believed could benefit from nutrition care than they could provide. In other words doctors probably came across patients that needed nutrition care than they could provide but did not refer them to dieticians/nutritionists. Doctors' ability to identify patients in need of nutrition care and subsequently referring them to dieticians/nutritionists is yet another process that could be used to improve patients' access to adequate nutrition care. Being gate keepers in the general practice setting essentially places doctors in a better position to actively facilitating and advocating for patient nutrition care.

\section{Conclusions regarding research question 3}

The nutrition practice behaviour of doctors was generally inadequate, although they recognised that a large proportion of their patients will benefit from nutrition care. Doctors' nutrition practice behaviour is hindered by barriers such as inadequate competence and self-efficacy in nutrition care and poor nutrition counselling skills. We found that quality and quantity of nutrition educational experiences of doctors is an important determinant of their nutrition practice behaviour. To improve nutrition practice behaviour, a critical step is to improve the nutrition educational experiences of medical students during medical school. Doctors generally refer patients to dieticians/nutritionists for further care, but the frequency does not meet the proportion of patients who may need such care. 


\section{Directions for future research}

1. Future studies should investigate the impact of nutrition education on the nutrition practice behaviour of doctors in the general practice setting.

2. It is unclear why doctors do not readily refer patients who they believe will benefit from nutrition care to dieticians, so future qualitative studies should explore this phenomenon.

\section{Research question 4}

What are the barriers to effective nutrition education and how could nutrition education be improved in the medical curricula? (Chapter 6)

It is imperative to find answers to two important questions: 1) why is nutrition education in the medical curriculum inadequate? 2) What can be done to promote effective nutrition education? The current literature regarding inadequacy of nutrition education is limited and largely echoes the perspectives and opinions of practicing doctors, teachers and researchers in health professions education ${ }^{1,22-24}$. The perspectives and opinions of medical students who experience the nutrition educational environment are lacking. Medical students are important stakeholders of the educational environment and are important sources of unique opinions and perspectives as to why their nutritional education is inadequate and unsatisfactory.

Following a constructivist qualitative research paradigm, the results of chapter 6 creates a knowledge base of medical students' perception, perspectives and opinions of the barriers to effective nutrition education and suggested strategies to improve nutrition education in the medical curriculum. Through semi-structured interviews, medical students provided their account of realities and meanings of barriers to effective nutrition education ${ }^{25}$. As reported in chapter 6 , barriers effective to nutrition education as perceived by these medical students can be classified into three broad areas: personal (which deals with the individual), interpersonal and environmental barriers (which deals with setting and context).

Nutrition education is not prioritised in the medical curriculum and has not been considered as a core component of medical training (chapter 6 ). This perception as held by medical educators over the years is partly responsible for the inadequate contact hours for nutrition education and nutrition content in the curriculum, notwithstanding the usual claim of the medical curriculum being overly crowded. As reported by medical students in chapter 6 , it is true that the medical curriculum is crowded, but it is also equally true and untenable that if curriculum planners and school authorities perceive nutrition education to be a priority and a critical aspect of medical training, an enabling environment will be created to improve learning in nutrition. 
The availability of faculty members who are trained in nutrition is key to effective nutrition education. Medical students need faculty to teach nutrition and to act as role models in nutrition care. This barrier is also mediated by faculty's lack of interest for further training in nutrition. As elaborated in chapter 6, unavailability of faculty trained in nutrition does not facilitate identification of appropriate nutrition content, coordination of teaching and learning activities in nutrition, promotion of active participation in nutrition education activities by both faculty and students, translation of nutrition science to clinical practice and promotion of role modelling of nutrition care at the hospital ${ }^{3,26-29}$.

If we want healthcare professionals to exhibit inter-professional care in the hospital setting, then we need to train them inter-professionally. It is thus consistent for medical students to discuss in chapter 6 that nutrition education is not effective due to poor collaboration with nutrition and other health professionals in their training. The apparent poor collaboration may not give students the opportunity to realise the multidisciplinary nature of nutrition care ${ }^{30}$ and will hinder inter-professional development and collaboration required for clinical care ${ }^{31}$.

Medical students' suggestion of integrating nutrition as a theme throughout the entire medical curriculum is consistent with previous reports ${ }^{23,32-34}$. Such a change will enable students to understand and realise the clinical application of nutrition in patient care. Fundamental to the success of this process is the execution of a needs assessment by way of a review of the curriculum to identify avenues that could be used for the integration of nutrition content throughout the curriculum.

\section{Conclusions regarding research question 4}

To the best of our knowledge, no study has qualitatively explored medical students' perceptions, perspectives and opinions of the barriers to effective nutrition education and suggested strategies to improve nutrition education. The barriers and strategies for effective nutrition education provided by these medical students are comparable to those reported by medical educators and practicing doctors. It is yet additional evidence supporting the widely accepted recognition of students, as important stakeholders of the curriculum, whose views should be tapped to improve the nutrition learning environment.

\section{Future research directions}

1. Further studies should be conducted among medical students from different settings to elucidate further our findings.

2. It is yet to be known whether the removal of the barriers will result in improved nutrition education. This could be considered in future studies.

3. The strategies suggested by the medical students towards improving nutrition education are at best perspectives and opinions until empirical studies are conducted to measure their effectiveness at improving nutrition education. It is thus important for 
future studies to evaluate the effectiveness of these strategies towards improving nutrition education in the medical curriculum.

\section{Research question 5}

How do practicing doctors perceive the effectiveness of the nutrition-related training and learning resources they are currently using and may use in the future? (Chapter 5)

Although medical students and practicing doctors have received inadequate nutrition education during medical school, they will still have to provide nutrition care since patients expect such care from them. Furthermore, patients consider them as important sources of nutrition information and will seek nutrition care from them, whether they were trained or not. Continuing nutrition education during practice presents an important opportunity for doctors to improve their competence and self-efficacy in nutrition care. There is evidence that in-service training programmes in nutrition can improve the nutrition competence and self-efficacy of practicing doctors as well as their nutrition practice behaviour ${ }^{35}$. However, the questions that remain unanswered are: Will doctors accept and participate in those programmes if organised? How do doctors perceive the effectiveness of such programmes? Are doctors currently using any nutrition-related training and learning resources to improve their competence in nutrition care? The results of Chapter 5 provide answers to these questions.

We showed in chapter 5 that the current nutrition competence and self-efficacy of practicing doctors is largely influenced by self-directed reading and learning. Medical students also indicated in Chapter 3 that self-directed reading and learning contributed to their current competence and self-efficacy in nutrition care. These findings, together with those of previous studies ${ }^{11}$, demonstrate that medical school training does not provide doctors with adequate nutrition education and that doctors will have to rely on other learning and training resources to improve their nutrition care competence to enable them meet the needs of patients.

The work-place setting (i.e., the hospital) is also an important nutrition-related training and learning resource, as practicing doctors reportedly said in Chapter 5 that clinical practice was their second most important contributor to their nutrition care competence and self-efficacy. The presence of faculty and superiors to model nutrition care in the clinic has been shown from previous studies to improve nutrition care competence and practice of nutrition care among practicing doctors ${ }^{27,36}$.

In Chapter 5, it was reported that doctors considered a number of continuing nutrition education training and learning resources to be effective. These included short nutrition seminars or lectures; online nutrition courses designed for doctors; dietician/nutritionist facilitated workshops for doctors; continuing medical education conferences on 
nutrition with continuing professional development (CPD) credits for participation; making access to nutrition-related journal articles; nutrition newsletters and nutrition information on patient handouts. Although doctors perceived these training and learning resources to be effective, only a few of them were currently using any of them for their continuing education in nutrition.

The low usage of these nutrition-related learning resources might be due to two reasons. Firstly, most of the training and learning resources listed are either non-existent or inadequate. If available, most doctors do not have access to them. Doctors will need to pay for subscriptions to online journal articles and also buy their own internet data bundles to have internet connectivity. Most hospitals do not have reliable internet connectivity and do not subscribe to important databases to get access to journal articles. Furthermore, nutrition CPD programmes, short nutrition seminars/courses and nutrition workshops facilitated by dieticians/nutritionists are non-existent. It presupposes that doctors make use of what may be available to them which is calling a dietician/nutritionist to consult. This resource (i.e. calling a dietician/nutritionist to consult) is also not readily available to all practicing doctors, since most hospitals do not have dieticians or nutritionist for them to rely upon. Secondly, practicing doctors may lack time to participate in such programmes, as has been reported previously.

Although medical doctors said they will adopt all the nutrition-related learning and training resources in the future, they were slightly more likely to participate in dietician/nutritionist facilitated workshops compared to other forms of nutrition-related teaching and learning resources. Consistent with these findings, medical students indicated in Chapter 4 that they would benefit from further training in nutrition care and that such training should come from a dietician or nutritionists. These findings demonstrate both medical students' and practicing doctors' recognition of the multidisciplinary nature of nutrition care and their acceptance of dieticians/nutritionists as important sources of nutrition education.

\section{Conclusions regarding research question 5}

Self-directed learning largely contributed to both doctors and medical students' current competence and self-efficacy in nutrition care. Doctors perceived a wide range of nutrition-related training and learning resources to be effective, but few of them were currently using any of them for their continuing education in nutrition. The findings of chapter 5 add to the literature that practicing doctors recognise that their training from medical school have not provided them adequate competence and self-efficacy in nutrition care, and as a result, practicing doctors are currently using learning and training resources available to them to improve their nutrition care competence. It also increases our understanding of practicing doctors' acceptability and readiness to participate in continuing nutrition education programmes. 


\section{Future research directions}

1. Appropriate continuing nutrition educational interventions are needed to allow the development of skills and confidence to support patients to make healthy dietary choices and to help prevent chronic diseases.

2. Future empirical studies should explore further doctors' reasons for low patronage of nutrition-related learning and training resources.

\section{Research question 6}

How to design a protocol to undertake a realist review of educational interventions to improve nutrition care competence and delivery? (Chapter 7)

The results of Chapters 2, 3, 4, 5 and 6 demonstrates that nutrition education is inadequate and that both medical students and practicing doctors feel inadequately prepared to provide nutrition care. This has resulted in both medical students and practicing doctors having inadequate knowledge, poor nutrition counselling skills and feeling less selfefficacious to provide nutrition care as reported in Chapters 3, 4 and 5 of this thesis. These findings demonstrate the need for the design of an educational intervention to improve nutrition care competence and practice. A number of educational interventions has been developed and implemented over the years, but often, the effects of these interventions have been inconsistent and weak ${ }^{37-39}$. There is thus the need to design interventions that can change healthcare professionals' nutrition care competence, self-care and practice behaviour ${ }^{37-39}$.

It is imperative to review the strengths and weaknesses of previous interventions, identifying success stories and failures as well as the contributing factors through a systematic review of the literature to inform the design of an intervention. Although a traditional systematic review would have been appropriate, such a review may not grant us the needed opportunity to explore and identify contributing factors. We thus adopted a relatively new and unique methodology, the realist synthesis/review (synthesis and review mean the same thing and are used interchangeably here). As presented in Chapter 7, traditional systematic reviews are known for their ability to evaluate the effectiveness of interventions using effect sizes. This is a short coming if the purpose of the review is "clarification research," which seeks to explore how and why interventions work to generate certain outcomes ${ }^{40}$, but not "justification research," which oversimplifies the inherent complexity and contextual nature of interventions as it seeks to minimise bias ${ }^{41,42}$. The latter, which relates to traditional systematic reviews, generates findings that do not account for complexity of interactions between conditions, mechanisms, and outcomes of interventions ${ }^{43}$. It is imperative to identify contextual factors, which mediate or inhibit 
their competence and delivery of nutrition care ${ }^{44,45}$. In order to meet those needs, researchers have to identify components of effective educational interventions and processes. The realist synthesis approach was appropriate to achieve these objectives.

Proposed by Pawson and Tilley ${ }^{46}$ and Pawson ${ }^{42}$, the realist approach is used for reviewing and synthesizing findings from complex interventions. It is based on the assumption that "no one theory fits it all" - i.e., no one theory can always explain or predict the outcomes in every context ${ }^{43,47}$. Precisely, the realist review is based on the principle that particular situations/context may influence particular human choices, which may result in certain semi-predictable behaviours (demi-regularities) ${ }^{42,43}$. In order to appreciate the theories underlying these demi-regularities, a realist review assesses the interaction between an intervention and the context of implementation and tries to expose and articulate the mechanisms that are triggered by this interaction to yield outcomes ${ }^{43,48,49}$ in a sample of primary studies. In the realist review and as adopted for this study, mechanisms are referred to as reactions/responses instigated by the intervention implemented within a particular situation/context resulting in certain outcomes ${ }^{48}$. In other words, "interventions provide resources which trigger choice mechanisms (M) which are taken selectively according to the characteristics and circumstances of subjects $(C)$ leading to a varied pattern of impact $(\mathrm{O})^{\prime \prime}{ }^{42}$. A realist review results in Context Mechanism Outcome Configurations (CMOCs) from the sample of primary studies. Serving as hypotheses in the realist approach, CMOCs are presentations of the interaction between the intervention, the context in which it is implemented and the processes (mechanisms) that are triggered by this interaction, resulting in the generation of an outcome ${ }^{48}$.

As presented in Chapter 7, we conceptualise that educational interventions and strategies to improve nutrition education, competence and nutrition practice behaviour to complex involving multiple actors (teachers, learners, patients, health care providers, etc.) operating at different levels in different environments and contexts ${ }^{43}$. Chapter 7 presents a protocol of how a realist review could be executed.

\section{Conclusions regarding research question 6}

Realist synthesis is a relatively new methodology in health profession education research and is arguably new in nutrition education research. To the best of our knowledge, this research is the first of its kind by using realist synthesis to review the literature on nutrition education. The protocol serves as a resource to inform and guide researchers in health professions education and nutrition, particularly those who may be interested in adopting the realist approach in future reviews. 


\section{Research question 7}

How, for whom, why, and in what circumstances do educational interventions work to improve nutrition care competence and nutrition practice behaviour among doctors and other healthcare professionals? (Chapter 8)

As indicated in Chapter 7, Chapter 8 is a realist synthesis of 46 educational interventions that aimed to improve nutrition care competence and nutrition practice behaviour of doctors and other healthcare professionals. The high numbers of studies included in the review demonstrate the vast numbers of primary studies of interventions designed to improve nutrition care competence and practice among healthcare professionals, including doctors. It also demonstrates broad and flexible nature of the realist approach to systematic reviews. The inclusion of other healthcare professionals was needed since our identification of educational interventions had nurses, pharmacist, and nutritionists/dieticians as sole participants or together with doctors. The findings of these studies were relevant to the objectives of the review (Chapter 8 ).

Given that realist synthesis is a theory-driven approach, the review was informed by an initial programme theory and a conceptual model that was revised in accordance with emerging evidence demonstrating the iterative nature of the approach.

An important finding of Chapter 8 was that educational interventions are likely to improve nutrition practice behaviour if they focused on developing favourable attitudes towards nutrition care and nutrition counselling skills. It became obvious in Chapter 8 that nutrition knowledge is important as it forms the basis for the other domains of competence (attitudes, skills) and self-efficacy, but is not sufficient to bring about change in nutrition practice behaviour since knowledge does not usually result in a change of behaviour. As demonstrated in Chapter 8, improvement in nutrition knowledge did not result in behaviour change of participants in a number of studies ${ }^{50,51}$. Interventions should thus focus on teaching and learning strategies that will encourage knowledge and skills development.

As presented in Chapter 6, it is an undeniable fact that barriers exist within the work and educational environments that hinder effective nutrition education and provision of nutrition care by doctors and other healthcare professionals. A doctor will not be able to provide nutrition care if the work environment does not support it. We thus found in Chapter 8 that educational interventions that minimised, removed or provided participants with ideas of how to overcome barriers within the work environment resulted in improved nutrition practice behaviour.

The results of Chapter 8 showed that reinforcement of outcomes gained from educational interventions that were administered to preclinical students is necessary to bring about improved nutrition practice behaviour after graduation. Seven interventions were administered to undergraduate medical students during their preclinical training. Most of these interventions were single required courses administered as a one-time event 
(usually during first and second year of medical education) without any opportunity for reinforcement. Although these interventions reported significant changes in nutrition knowledge $26,28,52-55$, self-reported nutrition counselling confidence, malnutrition diagnostic ability and students addressing nutrition in cardiovascular patients, the sustainability of these acquired competencies till practice were difficult in the absence of opportunities for reinforcement. The authors of such interventions were generally speculative of the effect of the interventions on healthcare professionals' nutrition practice behaviour in the future. As noted by Schlair et al. ${ }^{53}$, nutrition self-efficacy and nutrition care expectations may be difficult to address in the early preclinical years due to the lack of clinical exposure. Integration of nutrition content throughout the entire medical program with opportunities for clinical exposure and reinforcement may be more effective.

A multidisciplinary approach to teaching nutrition training was obvious and adopted in most of the educational interventions reviewed in Chapter 8. This approach was used in designing the course content to include multidisciplinary information and also informed the composition of the program planners and presenters. A multidisciplinary approach was designed to meet the cross-disciplinary nature of nutrition, which has been noted as one of the barriers to adequate nutrition education in Chapter 6. This approach allowed participants to recognise the multidisciplinary nature of nutrition care. It is commendable that almost all of the interventions reviewed had a nutritionist/dietician as part of the multidisciplinary team together with the concerned healthcare professional (physicians, nurses, nurse practitioners, etc). This finding is consistent with those in Chapter 3,4 and 5 that medical students and practicing doctors preferred to receive training in nutrition from dieticians/nutritionists.

Other important conditions of what works to facilitate the success of educational interventions included: adoption of appropriate and innovative teaching and learning strategies; interventions designed to meet the educational and clinical needs of doctors; using role modelling to build on the nutrition care self-efficacy, confidence and counselling skills of doctors; encouraging doctors to adopt healthy lifestyles and the use practical, local and relevant tools to encourage nutrition care.

As presented in our revised conceptual model, circumstances under which these may work to generate the needed short-, medium- and long-term outcomes include encouraging a multidisciplinary approach to nutrition education and nutrition care, as well as structuring healthcare systems that encourage the practice of nutrition care in the work environment by superiors. Similar to the findings of Chapters 3 and 6 , the recognition of nutrition as an important component of care delivery systems and the medical curricula is also a necessary condition. In a non-uniform fashion, these conditions together with the circumstances under which they work may interact to bring about improvement in both educational and clinical outcomes in the short, medium and long term. 


\section{Conclusions regarding research question 7}

To a large extent, we have demonstrated in Chapter 8 the characteristics of successful educational interventions to improve nutrition care competence and delivery. Educational interventions to improve nutrition care competence and practice of doctors and other healthcare professionals abound in the literature; however, few are designed and implemented in a manner that take cognisance of the context and ways in which those interventions work to bring about the desired outcomes. The strength of this review lies in its iterative and integrative nature allowing for the practical generation, testing and refinement of practical theories of how educational interventions work to bring about outcomes.

\section{Directions for future research}

1. Few of the studies that were reviewed adopted real-time, objective measures of change in nutrition practice behaviour, making it difficult to report unbiased outcomes. Future studies should adopt more objective measures of behaviour changes, such as objective structured clinical examinations and the use of incognito standardized patients.

2. Most of the studies did not investigate the influence of doctors' nutrition practice behaviour on clinical outcomes of patients'. Future studies should explore this phenomenon to support the important role of nutrition care in health and disease.

3. There is the need for more systematic reviews in the field of nutrition and health professions education using the realist approach.

4. Further work is needed to elucidate further the hypotheses, strategies and concepts generated in this review.

5. Authors of future educational interventions should endeavour to report the implementation challenges and contextual factors of interventions.

6. Given that few of the educational interventions had theoretical underpinnings, there is the need for authors of interventions to focus on the theoretical basis of such interventions and to explicitly demonstrate how theoretical mechanisms are defined and tested.

\section{Limitations of the studies of this thesis}

All the studies included in this thesis except for Chapter 7 and Chapter 8, followed a crosssectional design, which is data was collected at a point in time, making it difficult to draw conclusions informed by changes over time, which would require a longitudinal design. Furthermore, the cross-sectional nature makes it difficult to establish causal relationships. 
The studies used in this thesis relied on surveys and semi-structured interviews, which are self-reports of participants. Self-reports are liable to social desirability bias as well as recall bias. However, the self-critical responses provided by the participants and the use of previously validated items might have minimised effect of the social desirability bias.

This thesis is also limited by the focus of the studies on the views, opinions and perspectives of only medical students and to some extent that of practicing doctors. The non-inclusion of faculty did not allow for comparison and contrasting of views. Faculty are important stakeholders of medical curriculum, and their perspectives would have further enriched our findings.

The context in which the studies were conducted forms another important limitation. The findings were from one institution and from one country, making it difficult to generalize to other settings. Further studies are needed to replicate in different settings and context to elaborate further the generalisability of our findings.

Given that the findings of Chapter 8 were informed by the evidence of primary studies, it was a limitation that most of the primary studies provided limited, superficial descriptions of the context and mechanisms of the studies. Undoubtedly, this did not allow us to evaluate all the components of our candidate theories and to provide rich descriptions of the mechanisms identified. 


\section{References}

1. Ball LE, Hughes RM, Leveritt MD. Nutrition in general practice: role and workforce preparation expectations of medical educators. Aust J Prim Health 2010;16(4):304-310.

2. Ball L, Desbrow B, Leveritt M. An exploration of individuals' preferences for nutrition care from Australian primary care health professionals. Aust J Prim Health 2014;20(1):113-120.

3. Mogre V, Scherpbier AJ, Stevens F, Aryee P, Cherry MG, Dornan T. Realist synthesis of educational interventions to improve nutrition care competencies and delivery by doctors and other healthcare professionals. BMJ Open 2016;6(10):e010084.

4. Adams KM, Kohlmeier M, Zeisel SH. Nutrition Education in US Medical Schools: Latest Update of a National Survey. Acad Med 2010;85(9):1537-1542.

5. American Dietetic Association. Nutrition and you: Trends 2008. Report of Results. American Dietetic Association. 2008.

6. American Dietetic Association. Nutrition and you: Trends 2008. Report of Results. 2011.

7. Abramson S, Stein J, Schaufele M, Frates E, Rogan S. Personal exercise habits and counseling practices of primary care physicians: a national survey. Clin J Sport Med 2000;10(1):40-48.

8. Crowley J, Ball L, Han DY, et al. Doctors' attitudes and confidence towards providing nutrition care in practice: Comparison of New Zealand medical students, general practice registrars and general practitioners. J Prim Health care 2015;7(3):244-250.

9. Verma S, Paterson M, Medves J. Core competencies for health care professionals: what medicine, nursing, occupational therapy, and physiotherapy share. J Allied Health 2006;35(2):109-115.

10. Ball LE, Leveritt MD. Development of a validated questionnaire to measure the self-perceived competence of primary health professionals in providing nutrition care to patients with chronic disease. Fam Pract 2015;32(6):706-710.

11. Wynn K, Trudeau JD, Taunton K, Gowans M, Scott I. Nutrition in primary care Current practices, attitudes, and barriers. Can Fam Physician 2010;56(3):e109-e116.

12. Mihalynuk TV, Scott CS, Coombs JB. Self-reported nutrition proficiency is positively correlated with the perceived quality of nutrition training of family physicians in Washington State. Am J Clin Nutr 2003;77(5):1330-1336.

13. Bonevski B, Campbell E, Sanson-Fisher R. Primary care practitioners and health promotion: a review of current practices. Health Promot J Aust 1996;6(1):22.

14. Mogre V, Aryee PA, Stevens FC, Scherpbier AJA. Future Doctors' Nutrition-Related Knowledge, Attitudes and Self-Efficacy Regarding Nutrition Care in the General Practice Setting: A Cross-Sectional Survey. Med Sci Educ 2017;27(3):481-488

15. Hyska J, Mersini E, Mone I, et al. Assessment of knowledge, attitudes and practices about public health nutrition among students of the University of Medicine in Tirana, Albania. South East Euro J Public Health $2015 ; 1(1)$

16. Crowley J, Ball L, Han DY, Arroll B, Leveritt M, Wall C. New Zealand medical students have positive attitudes and moderate confidence in providing nutrition care: a cross-sectional survey. J Biomed Educ 2015;2015:7

17. Nowson CA, O'Connell SL. Nutrition knowledge, attitudes, and confidence of Australian general practice registrars. Journal of Biomedical Education. 2015;2015:6

18. Ray S, Rajput-Ray M, Ball L, et al. Confidence and attitudes of doctors and dietitians towards nutrition care and nutrition advocacy for hospital patients in Kolkata, India. Journal of Biomedical Education. 2015;2015:6

19. Kopelman P, Lennard-Jones J. Nutrition and patients: a doctor's responsibility. Clinical Medicine. 2002;2(5):391-394.

20. General Medical Council. Tomorrow's doctors: Outcomes and standards for undergraduate medical education. Manchester, UK: General Medical Council. 2009.

21. Kushner RF. Barriers to providing nutrition counseling by physicians: a survey of primary care practitioners. Prev Med 1995;24(6):546-552. 
22. Levine BS, Wigren MM, Chapman DS, Kerner J, Bergman R, Rivlin R. A national survey of attitudes and practices of primary-care physicians relating to nutrition: strategies for enhancing the use of clinical nutrition in medical practice. Am J Clin Nutr 1993;57(2):115-119.

23. St Jeor ST, Krenkel JA, Plodkowski RA, Veach TL, Tolles RL, Kimmel JH. Medical nutrition: a comprehensive, school-wide curriculum review. Am J Clin Nutr 2006;83(4):963S-967S.

24. Wong V, Millen BE, Geller AC, Rogers AE, Maury J-J, Prout MN. What's in store for medical students? Awareness and utilization of expert nutrition guidelines among medical school preceptors. Prev Med 2004;39(4):753-759.

25. McLellan L, Yardley S, Norris B, de Bruin A, Tully MP, Dornan T. Preparing to prescribe: How do clerkship students learn in the midst of complexity? Adv Health Sci Educ 2015;20(5):1339-1354.

26. Buchowski MS, Plaisted C, Fort J, Zeisel SH. Computer-assisted teaching of nutritional anemias and diabetes to first-year medical students. Am J Clin Nutr 2002;75(1):154-161.

27. Maiburg BHJ, Rethans JE, Schuwirth LWT, Mathus-Vliegen LMH, van Ree JW. Controlled trial of effect of computer-based nutrition course on knowledge and practice of general practitioner trainees. Am J Clin Nutr 2003;77(4S):1019S-1024.

28. Carson JAS, Gillham MB, Kirk LM, Reddy ST, Battles JB. Enhancing self-efficacy and patient care with cardiovascular nutrition eduction. Am J Prev Med 2002;23(4):296-302.

29. Taren DL, Thomson CA, Koff NA, et al. Effect of an integrated nutrition curriculum on medical education, student clinical performance, and student perception of medical-nutrition training. Am J Clin Nutr 2001;73(6):1107-1112.

30. Ball L, Crowley J, Laur C, Rajput-Ray M, Gillam S, Ray S. Nutrition in medical education: reflections from an initiative at the University of Cambridge. J Multidiscip Healthc 2014;7:209.

31. Bridges DR, Davidson RA, Odegard PS, Maki IV, Tomkowiak J. Interprofessional collaboration: three best practice models of interprofessional education. Med Educ Online 2011;16.

32. Krebs NF, Primak LE. Comprehensive integration of nutrition into medical training. Am J Clin Nutr 2006;83(4):945S-950S.

33. Kris-Etherton PM, Akabas SR, Bales CW, et al. The need to advance nutrition education in the training of health care professionals and recommended research to evaluate implementation and effectiveness. Am J Clin Nutr 2014;99(5):1153S-1166S.

34. Touger-Decker R. Nutrition education of medical and dental students: innovation through curriculum integration. Am J Clin Nutr 2004;79(2):198-203.

35. Sunguya BF, Poudel KC, Mlunde LB, Urassa DP, Yasuoka J, Jimba M. Nutrition training improves health workers' nutrition knowledge and competence to manage child undernutrition: a systematic review. Front Public Health 2013;1:37

36. Carson JAS, Gillham MB, Kirk LM, Reddy ST, Battles JB. Enhancing self-efficacy and patient care with cardiovascular nutrition education. Am J Prev Med 2002;23(4):296-302.

37. McLaren DS. Nutrition in medical schools: a case of mistaken identity. Am J Clin Nutr 1994;59(5):960-963.

38. Weinsier RL. National Dairy Council Award for Excellence in Medical/Dental Nutrition Education Lecture, 1995: medical-nutrition education--factors important for developing a successful programme. Am J Clin Nutr 1995;62(4):837-840.

39. Gershoff SN. National Dairy Council Award for Excellence in Medical/Dental Nutrition Education Lecture, 1996: nutrition education--success or failure? Am J Clin Nutr 1996;64(5):809-812.

40. Cook DA, Bordage G, Schmidt HG. Description, justification and clarification: a framework for classifying the purposes of research in medical education. Med Educ 2008;42(2):128-133.

41. Pawson R, Greenhalgh T, Harvey G, Walshe K. Realist synthesis: an introduction. ESRC Research Methods Programmeme. Manchester: University of Manchester. 2004.

42. Pawson R. Evidence-based policy: A realist perspective. Thousand Oaks (CA): Sage, 2006.

43. Wong G, Greenhalgh T, Pawson R. Internet-based medical education: a realist review of what works, for whom and in what circumstances. BMC Med Educ 2010;10(1):12.

44. Ball EL, Hughes MR, Leveritt DM. Nutrition in general practice: role and workforce preparation expectations of medical educators. Aust J Prim Health 2010;16(4):304-310. 
45. Mechanic D. Social research in health and the American sociopolitical context: the changing fortunes of medical sociology. Soc Sci Med 1993;36(2):95-102.

46. Pawson R, Tilley N. Realistic evaluation: Sage; 1997.

47. Shiell A, Hawe P, Gold L. Complex interventions or complex systems? Implications for health economic evaluation. BMJ 2008;336(7656):1281.

48. Kane SS, Gerretsen B, Scherpbier R, Dal Poz M, Dieleman M. A realist synthesis of randomised control trials involving use of community health workers for delivering child health interventions in low and middle income countries. BMC Health Serv Res 2010;10(1):286.

49. Greenhalgh T, Kristjansson E, Robinson V. Realist review to understand the efficacy of school feeding programme BMJ 2007;335(7625):858.

50. Ockene JK, Ockene IS, Quirk ME, et al. Physician training for patient-centered nutrition counseling in a lipid intervention trial. Prev Med 1995;24(6):563-570.

51. Dacey M, Arnstein F, Kennedy MA, Wolfe J, Phillips EM. The impact of lifestyle medicine continuing education on provider knowledge, attitudes, and counseling behaviors. Medical teacher. 2013;35(5):e1149-e1156.

52. Afaghi A, Mohamadi AAHA, Ziaee A, Sarchami R. Effect of an Integrated Case-based Nutrition Curriculum on Medical Education at Qazvin University of Medical Sciences, Iran. Glob J Health Sci 2012;4(1):112.

53. Schlair S, Hanley K, Gillespie C, et al. How medical students' behaviors and attitudes affect the impact of a brief curriculum on nutrition counseling. J Nutr Educ Behav 2012;44(6):653-657.

54. Kohlmeier M, Althouse L, Stritter F, Zeisel SH. Introducing cancer nutrition to medical students: effectiveness of computer-based instruction. Am J Clin Nutr 2000;71(4):873-877.

55. Roche PL, Ciccarelli MR, Gupta SK, Hayes BM, Molleston JP. Multi-School Collaboration to Develop and Test Nutrition Computer Modules for Pediatric Residents. J Am Diet Assoc 2007;107(9):1586-1589. 

Summary 



\section{Nutrition care and its education: medical students' and doctors' perspectives}

A PhD thesis that investigated medical students' and doctors' perspectives regarding the role of nutrition care in medical education; nutrition education in the medical curriculum; nutrition care competencies and practice behaviour; barriers to nutrition education and care and educational interventions on how to improve nutrition education and provision of nutrition care.

\section{Introduction}

Many diseases in the domain of public health have poor nutrition as an underlying cause. Nutrition is even more important for the sub-Saharan Africa region, which is characterized by rising levels of under nutrition and non-communicable diseases (NCDs), a situation of the double burden of malnutrition.

Encouraging individuals to adopt healthy nutrition and dietary habits is thus an important step towards improving health and preventing disease. Nutrition educators, specialists and multidisciplinary support teams are necessary to support individuals in adopting good nutrition and dietary habits. Among all the identifiable human resources, medical doctors are regularly considered as credible sources of nutrition advice and are better placed to provide dietary and nutrition advice to patients.

Doctors, however, do not readily provide nutrition care to their patients. They regularly report being inadequately trained and unprepared to provide nutrition care. A number of factors have contributed to this situation, including poor nutrition education during medical school, lack of role models to model nutrition care, lack of time, poor reimbursement and low priority for nutrition education, among others.

The evidence regarding nutrition education and poor nutrition care among doctors largely originate from developed countries such as the US and other developed countries. Evidence from countries in sub-Saharan Africa, such as Ghana, is limited. The peculiarity of the educational setting and health system of the sub-region requires specific evidence to inform the design of interventions to meet its specific needs.

The chapters of this thesis focus on nutrition education in undergraduate medical curriculum as well as the provision of nutrition care in the general practice setting. Through the chapters, we present findings obtained from both quantitative and qualitative research executed from 2014 to 2018 to provide insights into the adequacy of nutrition education in the undergraduate medical curriculum, barriers and strategies to effective nutrition education, the practice of nutrition care in the general practice setting and the characteristics of educational interventions designed to improve nutrition care competencies and delivery. 
The general research question of this thesis is: What is the current practice of a) nutrition education for medical students and b) nutrition care of practicing doctors in Ghana?

Specifically, the chapters of the thesis provide answers to the following research questions:

1. How do medical students perceive the role of nutrition care in their training, and what do they perceive to be the roles and responsibilities of doctors in the provision of nutrition care?

2. What are the nutrition care competence and self-efficacy of medical students and practicing doctors?

3. What are practicing doctors' nutrition care practices and referral behaviours in the general practice setting, and what are the barriers to the provision of nutrition care?

4. What are the barriers to effective nutrition education, and how could nutrition education be improved in medical curriculum?

5. How do practicing doctors perceive the effectiveness of the nutrition-related training and learning resources that they are currently using and may use in the future?

6. How to design a protocol to undertake a realist review of educational interventions to improve nutrition care competence and delivery?

7. How, for whom, why and in what circumstances do educational interventions work to improve nutrition care competence and nutrition practice behaviour among doctors and other healthcare professionals?

\section{Chapter 1}

As an introductory chapter, Chapter 1 provides a brief background to the importance of nutrition in health and disease and how poor nutrition is an important contributor to the rising prevalence of non-communicable diseases (NCDs) and premature deaths around the globe and in sub-Saharan Africa, specifically. While the adult population of the subSaharan Africa region is affected by increasing prevalence of excess bodyweight and cardiovascular diseases, its children are troubled by under nutrition and infection. Nutrition care is key to the design of interventions to reduce the rising prevalence of these diseases of the double burden of malnutrition. The chapter also addresses the concept of nutrition care competency and the nutrition care process. We conceptualise in chapter 1 that competencies such as knowledge, attitudes, skills and self-efficacy are necessary for doctors to exhibit the behaviour of providing nutrition care to patients in the general practice setting. The nutrition care process as used in dietetic care is a distinct interrelated series of steps, and we conceptualise that the doctor has a role to play in these steps - i.e., nutrition assessment, nutrition diagnosis, nutrition intervention, nutrition monitoring and evaluation. A description of the literature of why doctors should provide nutrition care and the status of nutrition education, based on evidence from previous studies, is 
also addressed in Chapter 1. The literature identifies that nutrition education is inadequate in the medical curriculum and doctors' provision of nutrition care is sub-optimal. However, this evidence comes from the settings of developed countries which may not be adequate to inform the design of interventions for the settings in sub-Saharan Africa.

\section{Chapter 2}

The qualitative research presented in Chapter 2 explores medical students' views and perspectives of why they as medical students should learn about nutrition and why doctors should provide nutrition care to patients. It explored medical students' perspectives of which healthcare professional should provide nutrition care to patients. Medical students discussed and recognised that all health professionals have a role to play in the provision of nutrition care to patients in the general practice setting. Medical students opined that doctors should play a more active role than other health professionals due to the following reasons: patients having regular contacts with the doctor; doctors being the first point of contact for patients to consult and patients having more trust in the doctors' advice. In conclusion, medical students recognised the need for them to learn about nutrition care and for doctors to play a role in the provision of nutrition care.

\section{Chapter 3}

The results of Chapter 3 addressed three important areas regarding nutrition education and nutrition care: relevance of nutrition education to medical students' practice; perceived satisfaction with their current nutrition education environment (i.e., quantity and quality) and their perceived preparedness to provide nutrition care. Augmenting the findings of Chapter 2, we found that medical students perceived nutrition education to be highly relevant to their future practice as medical doctors. However, they were unsatisfied with their current nutrition educational experiences and reportedly felt unprepared to provide nutrition care to patients in a general practice setting. We also found that medical students' perception of the quality and quantity of their nutrition education correlated with their perceived preparedness to provide nutrition care. These findings provide evidence that suggest that nutrition education is inadequate in Ghana. Yet again, the findings suggest that improving the quality and quantity of the nutrition education in medical school may result in improved preparedness to provide nutrition care, which may subsequently result in improved nutrition practice behaviour. 


\section{Chapter 4}

Building on the findings of Chapter 3, Chapter 4 focused on nutrition care competencies and self-efficacy of medical students. This chapter also investigated the association between nutrition-related knowledge, attitude and self-efficacy. We also investigated whether medical students' nutrition care competencies differed in their level of clinical training. Medical students' nutrition-related knowledge regarding malnutrition in children was low. Their nutrition-related mean knowledge scores in the nutrition topics assessed differed by their level of clinical training. Students in the early part of their clinical training scored higher than their counterparts in the latter part of their clinical training in topics that related to basic nutrition concepts. Regarding nutrition topics that had a clinical orientation, medical students in the latter part of their training scored higher than those in their early part of their training. Clinical exposure and learning nutrition without reinforcement in the early part of medical training might have contributed to the differences in nutrition-related knowledge according to medical students' level of clinical training.

Although medical students had positive attitudes towards nutrition care, half of them were not sure of the effectiveness of health education in promoting patient's adherence to healthy lifestyle recommendation. Furthermore, medical students were less self-efficacious in their ability to provide certain aspects of nutrition care to patients. Medical students perceived self-efficacy as correlated with their attitudes towards nutrition care.

The findings in this chapter can be said to depict inadequacy of nutrition education in the medical curriculum and provides more reasons why nutrition education should be improved in medical education.

\section{Chapter 5}

Having assessed the views of students regarding their current nutrition education in the medical curriculum, nutrition care competencies and self-efficacy care, Chapter 5 focused on practicing doctors' perspectives of their nutrition education during medical school as well as their nutrition practice behaviour. Specifically, we evaluated medical doctors' nutrition care practices, competencies and barriers to providing nutrition care. Furthermore, medical doctors' satisfaction and perceived adequacy of their nutrition education, as well as the use and effectiveness of training and learning resources for continuing nutrition education, were assessed.

Medical doctors' level of comfort when discussing nutrition topics with their patients was just average; however, they reportedly felt more comfortable discussing topics relating to the nutrition of mothers and children compared with those of general nutrition and nutrition in chronic disease. Similar to reports among medical students, medical doctors had positive attitudes towards nutrition care in that they (1) considered nutrition care to be one of their responsibilities, (2) identified nutrition to be important in prevention and 
treatment of chronic diseases and (3) believed that their patients will adopt healthy lifestyles if counselled to do. They noted that their patients needed more nutrition information and nutrition care than they were able to provide.

As reported among medical students in Chapter 4, practicing doctors' attitude towards nutrition care correlated with their level of comfort in providing nutrition care. Comfort levels were associated with nutrition practice behaviour as doctors who had higher comfort levels were more likely to estimate that a greater proportion of their patients actually received nutrition care from them.

Medical doctors recognised the role of the dietician/nutritionist and promoted interprofessional care as the majority of them reportedly referred patients to dieticians/nutritionists for specialist dietary care or consulted these healthcare professionals for advice.

Despite the positive attitudes towards nutrition care, medical doctors' actual provision of nutrition care was below expectations in the sense that their estimate of the proportion of patients who actually received nutrition care from them fell below their estimate of the proportion of patients that the medical doctors believed will benefit from nutrition care. Furthermore, majority of doctors reportedly spent less than six minutes discussing nutritional issues with their patients. A number of barriers hindered doctors' ability to meet the expectations of their patients regarding nutrition care. These were lack of time, inadequate knowledge and counselling skills. It is unsurprising that inadequate knowledge and poor counselling skills have been identified by practicing doctors as barriers that hindered their provision of nutrition care, since more than half of them said they had inadequate nutrition education during medical school and more than threequarters were either unsatisfied or undecided regarding the quality and quantity of their nutrition education during medical school. These findings are similar to those of the medical students reported in Chapters 3 and 4.

Noting the important role of quality and quantity of nutrition education during medical school towards improving nutrition practice behaviour, we reported in Chapter 5 that doctors who said their nutrition education was adequate, as well as those who felt more satisfied with their nutrition educational experiences during medical school, were more likely to report being more comfortable discussing nutrition topics with their patients. Furthermore, practicing doctors who perceived their nutrition education to be inadequate significantly reported spending two minutes or less counselling patients about nutrition. These findings demonstrate the important role of improving nutrition education in the medical curriculum.

Being conscious of their deficiencies regarding nutrition care and education, medical doctors resorted to self-directed reading and clinical practice to improve their nutrition care competencies. Interestingly, a little below 50\% of the doctors were currently using any nutrition-related learning and training resource for their continuing education in nutrition, although over $80 \%$ found them to be effective and will be using them in the future if those learning and training resources are made available to them. 
In conclusion, medical doctors consider nutrition care to be an important responsibility, but they are not able to adequately provide such care to their patients due to inadequate nutrition education during medical school, a lack of time and inadequate knowledge, confidence and counselling skills.

\section{Chapter 6}

Adopting a qualitative approach, Chapter 6 explored medical students' perspectives on doctors' role in nutrition care, barriers and strategies to improving nutrition education in medical curriculum. The findings of this chapter are informed by semi-structured interviews of 23 clinical level medical students from the University for Development Studies, School of Medicine and Health Sciences (UDS-SMHS). Medical students discussed that the doctors' role in nutrition care should be supportive or complementary to that of the nutritionist/dietician. They further opined that doctors should be able to identify patients in need of nutrition care, provide some form of basic nutrition care and/or refer the patient to a dietician/nutritionist for specialist dietary care. According to medical students, doctors should be able to advocate for nutrition care for their patients and should also be able to monitor the progress of the patient regarding nutrition care.

Having identified these roles, they opined that their nutrition education in medical school is inadequate (similar to those of chapter 3,4 and 5) making them unconfident and probably unable to meet these roles they have identified.

Medical students go further to provide barriers that are hindering the effective provision of nutrition education in medical curriculum. These barriers were grouped into three categories: personal, interpersonal and environmental barriers. Interpersonal barriers concern the individual faculty member or curriculum planner, and examples include faculty's lack of knowledge in clinical nutrition, faculty's lack of motivation and interest to teach nutrition and perception of curriculum planners and faculty members that nutrition care is not the responsibility of doctors. As discussed by medical students, interpersonal barriers related to poor collaboration among medical educators and nutritionists/dieticians to promote teaching and learning in nutrition education. Environmental barriers were dependent on the setting, policies and organisation structure of medical schools. Examples of such barriers were: poor integration of nutrition education into the curriculum, time constraints, and lack of faculty to provide nutrition education and an already overcrowded curriculum, among others. Notwithstanding the fact that these are medical students who are undergoing training, the barriers that they identified are important, unique and some are consistent with those provided by medical educators, researchers in health professions education and medical doctors in previous studies.

Medical students did not only discuss barriers, but also suggested strategies that could be used to overcome or minimise some of the barriers to effective nutrition education. Some of these strategies included: the early incorporation of nutrition as a theme 
and throughout the entire curriculum; increasing faculty, medical educators' and curriculum planners' awareness that doctors have a responsibility to play in the provision of nutrition care; reviewing the curriculum to identify opportunities to incorporate and integrate nutrition education and collaborating with dieticians/nutritionist to teach nutrition in the medical curriculum.

The strategies provided by these medical students are comparable with those of previous findings, and if implemented, they could help bridge the nutrition education gap in the medical curriculum.

These findings demonstrate how medical students could serve as important sources of information that should be tapped to improve the nutrition education environment in the medical curriculum. The main conclusion from this chapter is that nutrition education is fraught with several barriers, and that the situation could be improved if faculty, curriculum planners and relevant stakeholders consciously perceive nutrition education to be a priority and an important role of the medical doctor.

\section{Chapters $7 \& 8$}

These two chapters are closely related, and that he first chapter serves as a stepping stone for the next. Having investigated the situation of nutrition education, doctors and medical students' competencies in nutrition care, and barriers to effective nutrition education, chapters $7 \& 8$ reviewed the literature to identify the characteristics of educational interventions that have been designed to improve nutrition education and care.

Chapter 7 is a protocol that outlines the methods, procedures and processes used in conducting the review. The review follows a realist approach that aims to identify the context and mechanisms of interventions and how they work to generate outcomes. This approach is relatively new in health professions education and nutrition research, and the protocol serves as guide for future reviews that intends to adopt the realist approach.

Chapter 8 thus reviews the literature to determine what, how, for whom, why and in what circumstances educational interventions to improve the delivery of nutrition care by doctors and other healthcare professionals work? Evidence from the review in chapter 8 indicated that for interventions to generate the needed outcomes, it is important to undertake a needs assessment to identify gaps in learners' knowledge or practice behaviour, and how they learned best, to inform the content, format and design of curricula.

To improve the delivery of nutrition care, it is essential for researchers to develop and design interventions that will encourage the acquisition of skills and attitudes and put less emphasis on cognitive gains, such as knowledge. It became apparent in the review that knowledge gains do not necessarily result in a change in nutrition practice behaviour, but improving skills, attitudes and self-efficacy may be more important to bring about change of behaviour. Future interventions should thus incorporate teaching and learning strategies that will bring about the acquisition of these outcomes. 
Findings of Chapter 8 demonstrated that the presence of role models and superiors modelling nutrition care in the hospital setting was found to promote improved nutrition practice behaviour. Seeing superiors' model nutrition care led research participants to feel more confident, accepted and credible. They anticipated their actions being valued, which led them towards changing their nutrition practice behaviour.

We also found in chapter 8 that interventions will work to bring about change in nutrition practice behaviour if they addressed structural and systematic barriers that hindered the provision of nutrition care. Some of the strategies adopted by these interventions included: restructuring of office environments to make them more conducive to providing nutrition care; creating an environment in which doctors could utilize their knowledge in nutrition; providing nutritional messages that busy doctors could deliver to patients and collaborating between education and care delivery.

In Chapter 8, we found that providing doctors with local, practically relevant tools and messages relating to nutrition and healthy dietary habits encouraged doctors to provide nutrition care to their patients. These type of interventions adopted strategies that simplified nutrition information that can easily be understood by patients. Some of the strategies adopted by these interventions were the use of memorable slogans, simple 'key take home messages', personalized nutrition messages, and locally relevant examples. Those interventions helped professionals engage in specific rather than generic discussions with patients, and provide advice and recommendations that patients found relevant.

Our findings in chapter 8 also demonstrated the important role of the personal health habits of the doctor in the provision of nutrition care. We found that interventions that encouraged doctors and other healthcare providers to adopt healthy dietary habits had positive outcomes - i.e., resulted in improved nutrition practice behaviour. Those who adopted healthy dietary habits felt confident to advise patients on their nutrition as they considered themselves as role models.

Information communication technology (ICT) through computers and online learning tools present important opportunities to improve nutrition education during medical school and during practice. We found in chapter 8 that ICT based education allowed for easy updating of content, permitted self-directed and independent study of nutrition information, presented content consistently, were accessible, and promoted interactivity. Interventions that adopted this approach were also convenient for participants because they were self-administered and self-paced. Interventions that were ICT-based reported improved nutrition knowledge gains, positive attitudes and improved nutrition practice behaviour among participants.

Other characteristics of successful educational interventions that yielded positive outcomes as reported in chapter 8 included: using innovating teaching and learning strategies and improving participants' self-efficacy in the provision of nutrition care.

Given that the realist approach is theory driven, the review was informed by a programme theory which was subsequently revised in light of the evidence adduced during 
the review. An important revision of the initial programme theory was the conception of "outcome' of interventions as short-, medium- and long-term outcomes. The initial theory did not conceive outcomes into these hierarchal levels, as it had only one outcome which is delivery of nutrition care. However, the evidence of the review demonstrated that delivery of nutrition care may be a medium outcome which is dependent on the attainment of short-term outcomes, such as knowledge, positive attitudes and self-efficacy or confidence. The long-term outcome, which is improved clinical outcomes among patients, can be realized with a change in nutrition practice behaviour that is enhanced by certain conditions/contexts such as restructuring the healthcare system, removing structural and systemic barriers, adopting favourable policies for nutrition care, providing appropriate tools to deliver nutrition care, investing more in preventive care and providing an office that makes it easier to provide nutrition care.

In conclusion, the findings demonstrate that educational interventions should go beyond the simple acquisition of knowledge and strive to improve participants' attitudes, skills and self-efficacy. The findings provide important insights into key areas that should be considered in interventions as well as the kind of teaching and learning strategies that could be adopted to improve nutrition education and nutrition practice behaviour.

\section{Chapter 9}

In this last chapter we discussed the research questions that informed the research project, situating the findings within relevant literature, making conclusions and suggesting future research directions. In addition, the strengths and limitations of the research are also presented.

In answering research question 1 , we found that medical students recognised the important role of nutrition care in health and disease and the need for them to learn about nutrition. Medical students provided important reasons why doctors should play an important role in nutrition care, and these reasons included the fact that doctors drive the healthcare system in Ghana and also play the role of gate keepers of healthcare.

For research question 2, both medical students and practicing doctors felt inadequately prepared to provide nutrition care, and although they have positive attitudes towards nutrition care, they reportedly felt inadequate in nutrition-related knowledge and less self-efficacious or confident to provide nutrition care to patients. However, more extensive studies are needed from other parts of Ghana and the sub-Saharan African region to further illuminate our findings.

In providing answers to research question 3, practicing doctors were unable to provide nutrition care to all patients they felt needed such care due to barriers, such as inadequate knowledge, lack of time and inadequate counselling skills. The quality and 
quantity of nutrition education received by practicing doctors, as well as medical students, is an important determinant of preparedness to provide nutrition care and nutrition practice behaviour. Future studies can elucidate further this finding.

Answers to research question 4 led to medical students discussing barriers to effective nutrition education in the medical curriculum. Medical students identified important barriers that hinder the effective provision of nutrition education, some of which included an already overcrowded curriculum, lack of priority for nutrition education and poor collaboration among medical educators and dieticians/nutritionists, among other barriers.

Answers to research question 5 brought to the fore that both medical students and practicing doctors rely on self-directed learning to improve their competencies and selfefficacy in nutrition care, as they found that their nutrition education in medical school was inadequate. A demonstration of practicing doctors' readiness to learn more about nutrition was also established.

Research questions 6 and 7 demonstrate the use of realist synthesis to review the literature identifying the characteristics of successful educational interventions designed to improve nutrition care competency and delivery of nutrition care. The protocol of the realist synthesis serves as a guideline for the design of similar reviews in health professions education and nutrition research.

In general, the findings of this thesis are limited by the cross-sectional nature of the studies, the use of surveys that relied on self-reports and the majority of the studies originating from a single institution. However, the findings present important implications for medical education. The findings serve as basis for the reforms and innovation in the medical curriculum in Ghana. Nutrition education warrants more attention as the evidence demonstrates that it is inadequate. There is also the need to begin to identify and align the role of the doctors regarding nutrition care. Incorporating nutrition as a theme throughout the entire medical curriculum is an important way to improve nutrition education. 
Samenvatting 



\section{Voedingszorg in het onderwijs vanuit het perspectief van geneeskundestudenten en artsen}

Een proefschrift dat de rol van voedingszorg tijdens de medische opleiding onderzoekt vanuit het perspectief van studenten en artsen; voedingsonderwijs tijdens de medische opleiding; competenties in voedingszorg en voedingszorg in de praktijk; belemmeringen voor voedingsonderwijs en voedingszorg en onderwijskundige interventies ter verbetering van het voedingsonderwijs en de voedingszorg.

\section{Inleiding}

Veel ziekten in het domein van de volksgezondheid worden mede veroorzaakt door slechte voeding. Voeding is een nog belangrijkere factor voor de Sub-Saharaanse regio in Afrika, die wordt gekenmerkt door toenemende ondervoeding en niet-overdraagbare ziekten (zoals obesitas en diabetes), waardoor er sprake is van de zogenaamde dubbele last van ondervoeding.

Een belangrijke stap in het verbeteren van de gezondheid van mensen en het voorkomen van ziekten is dan ook het bevorderen van een gezonder eetpatroon. Voedingsdocenten, specialisten en multidisciplinaire ondersteunende teams moeten mensen daarbij helpen. Van alle denkbare deskundigen worden artsen meestal als de meest geloofwaardige voedingsadviseurs gezien. Zij bevinden zich immers in de beste positie om patiënten over voeding te adviseren.

Artsen zullen echter niet zo snel voedingszorg leveren aan hun patiënten. Zij geven vaak aan dat ze daarvoor onvoldoende zijn opgeleid en daarop niet goed zijn voorbereid. Daar ligt een aantal factoren aan ten grondslag, zoals ontoereikend voedingsonderwijs tijdens de medische opleiding, de afwezigheid van rolmodellen om de voedingszorg vorm te geven, tijdgebrek, geldgebrek en weinig aandacht voor voedingsonderwijs.

Onze kennis over voedingsonderwijs en over de beperkte voedingszorg door artsen is grotendeels afkomstig uit ontwikkelde landen zoals de VS. Er is maar beperkte informatie beschikbaar over landen in Sub-Saharaans Afrika, zoals Ghana. Omdat het onderwijs en de gezondheidszorg in deze regio sterk afwijken van die in de ontwikkelde landen is er specifiek onderzoek nodig om interventies te kunnen ontwikkelen die aansluiten op de specifieke behoeften in deze regio.

De hoofdstukken van dit proefschrift gaan over voedingsonderwijs in de eerste jaren van de medische opleiding en over het leveren van voedingszorg in de praktijk. We presenteren de resultaten van het onderzoek (kwantitatief en kwalitatief) dat we tussen 2014 en 2018 hebben uitgevoerd en bieden daarmee inzicht in het niveau van het voedingsonderwijs in de eerste jaren van de medische opleiding, in factoren die effectief voedingsonderwijs in de weg staan en strategieën om daar iets aan te doen, in voedings- 
zorg in de praktijk en de kenmerken van onderwijskundige interventies waarmee de competenties op het gebied van voedingszorg en het leveren van deze zorg kunnen worden verbeterd.

De algemene onderzoeksvraag van dit proefschrift luidt: Wat is de huidige stand van zaken van a) het voedingsonderwijs aan geneeskundestudenten en b) de voedingszorg door artsen in Ghana?

In de hoofdstukken van dit proefschrift proberen we de volgende deelvragen te beantwoorden:

1. Hoe oordelen geneeskundestudenten over de rol van voedingszorg tijdens hun opleiding en welke taken en verantwoordelijkheden hebben artsen volgens hen in het leveren van voedingszorg?

2. Welke competenties hebben geneeskundestudenten en artsen als het om voedingszorg gaat en in hoeverre zijn zij zelfredzaam?

3. In welke mate leveren artsen tijdens hun praktijkwerk voedingszorg of verwijzen zij mensen door, en welke belemmeringen staan het leveren van voedingszorg in de weg?

4. Welke belemmeringen staan effectief voedingsonderwijs in de weg en hoe kan het voedingsonderwijs binnen de medische opleiding worden verbeterd?

5. Hoe oordelen artsen over de effectiviteit van voedingsgerelateerde trainingen en informatiematerialen die zij op dit moment inzetten of in de toekomst wellicht gaan gebruiken?

6. Hoe kun je een protocol ontwerpen waarmee een realistisch oordeel kan worden geveld over onderwijskundige interventies ter verbetering van de (competenties in) voedingszorg?

7. Hoe kunnen onderwijskundige interventies ervoor zorgen dat artsen en andere zorgverleners bekwamer worden op het gebied van voedingszorg en meer voedingszorg gaan leveren, en voor wie, waarom en in welke omstandigheden is dit zinvol?

\section{Hoofdstuk 1}

Het inleidende eerste hoofdstuk geeft beknopte achtergrondinformatie over het belang van voeding als het gaat om gezondheid en ziekte. Overal ter wereld, maar met name in Sub-Saharaans Afrika, speelt slechte voeding een belangrijke rol in de toename van nietoverdraagbare ziekten en vroegtijdige sterfte. De volwassen bevolking van de Sub-Saharaanse regio krijgt steeds vaker te maken met overgewicht en hart- en vaatziekten, terwijl hun kinderen last hebben van ondervoeding en infectieziekten. Voedingszorg is een cruciale factor bij het ontwikkelen van interventies waarmee de toename van deze ziekten, die het gevolg zijn van de dubbele last van ondervoeding, kan worden teruggedrongen. Dit hoofdstuk gaat ook in op het begrip competentie in voedingszorg en het proces van voedingszorg. Verder zetten we in Hoofdstuk 1 uiteen dat artsen over competenties als 
kennis, een positieve houding, vaardigheden en zelfredzaamheid moeten beschikken om in hun dagelijkse praktijk voedingszorg aan patiënten te kunnen leveren. Het proces van voedingszorg zoals dat in de dieetzorg wordt toegepast verloopt volgens een aantal duidelijk herkenbare, met elkaar samenhangende stappen. Wij laten zien dat artsen in deze stappen - beoordeling, diagnose, interventie, monitoring en evaluatie - een rol moeten spelen. Daarnaast beschrijven we in Hoofdstuk 1 wat er in de bestaande onderzoeksliteratuur wordt gezegd over het leveren van voedingszorg door artsen en de stand van het voedingsonderwijs. Uit de literatuur blijkt dat het voedingsonderwijs op medische opleidingen niet toereikend is en dat de mate waarin artsen voedingszorg bieden niet optimaal is. Deze informatie is echter gebaseerd op onderzoek in ontwikkelde landen en is daarom wellicht niet geschikt om als basis te dienen voor interventies in Sub-Saharaans Afrika.

\section{Hoofdstuk 2}

Het kwalitatieve onderzoek dat in Hoofdstuk 2 wordt gepresenteerd gaat over het oordeel van geneeskundestudenten over de vraag waarom zij voedingsonderwijs zouden moeten krijgen en waarom artsen voedingszorg zouden moeten leveren aan patiënten. De studenten werd gevraagd welke zorgverleners naar hun mening voedingszorg aan patiënten moeten bieden. De geneeskundestudenten waren van mening dat alle zorgverleners daarin een rol moeten vervullen. En ze vonden dat artsen een actievere rol moeten hebben dan andere zorgverleners, om de volgende redenen: patiënten hebben regelmatig contact met hun arts, artsen zijn het eerste aanspreekpunt voor patiënten en patiënten hebben meer vertrouwen in het advies van hun arts. Kortom, geneeskundestudenten vonden dat voedingszorg onderdeel moet zijn van het curriculum en dat artsen een rol moeten spelen in het leveren van voedingszorg.

\section{Hoofdstuk 3}

Hoofdstuk 3 gaat over drie belangrijke aspecten van voedingsonderwijs en voedingszorg: de relevantie van voedingsonderwijs voor geneeskundestudenten, de mate waarin zij tevreden zijn over hun huidige voedingsonderwijs (zowel kwantitatief als kwalitatief) en de mate waarin zij bereid zijn voedingszorg te leveren in de praktijk. Als aanvulling op de resultaten uit Hoofdstuk 2 bleek dat geneeskundestudenten van mening waren dat voedingsonderwijs zeer relevant is voor hun toekomstige werk als arts. Zij waren echter niet tevreden met hun huidige onderwijs over voeding en gaven aan niet goed voorbereid te zijn op het leveren van voedingszorg aan patiënten in de praktijk. Verder kwam naar voren dat er een correlatie bestaat tussen het oordeel van de studenten over de kwaliteit en kwantiteit van hun voedingsonderwijs en de mate waarin zij (voor)bereid zijn om voedingszorg te leveren. Deze resultaten laten zien dat het voedingsonderwijs in Ghana niet 
toereikend is en dat een kwalitatieve en kwantitatieve verbetering van het voedingsonderwijs tijdens de medische opleiding kan leiden tot een grotere bereidheid om voedingszorg te leveren, waardoor de voedingszorg in de praktijk ook kan verbeteren.

\section{Hoofdstuk 4}

Voortbouwend op de resultaten van Hoofdstuk 3 hebben we ons in Hoofdstuk 4 geconcentreerd op competenties in voedingszorg en zelfredzaamheid van geneeskundestudenten. In dit hoofdstuk hebben we ook gekeken naar de relatie tussen kennis over voeding, houding en zelfredzaamheid. Ook onderzochten we in hoeverre de competenties van de studenten op het gebied van voedingszorg afhankelijk waren van in welke fase van de klinische opleiding ze zich bevonden. De geneeskundestudenten hadden niet veel kennis over ondervoeding bij kinderen. Hun gemiddelde scores bij de vragen over voeding bleken afhankelijk te zijn van in welke fase van de klinische opleiding ze zich bevonden. Studenten die aan het begin stonden van hun klinische opleiding scoorden hoger op voedingsgerelateerde kennis dan hun studiegenoten in de latere fase van hun klinische opleiding. Maar bij voedingsgerelateerde onderwerpen die meer klinisch georiënteerd waren, scoorden studenten in de latere fase van hun opleiding juist hoger dan hun studiegenoten die nog aan het begin van hun opleiding stonden. De hoeveelheid klinische ervaring en voedingskennis die in de beginfase van de medische opleiding wordt opgedaan zonder dat het geleerde wordt verankerd zouden kunnen verklaren waarom de voedingsgerelateerde kennis van studenten per opleidingsfase verschilt.

Hoewel geneeskundestudenten positief tegenover voedingszorg stonden, betwijfelde de helft of gezondheidsonderwijs ertoe kan bijdragen dat patiënten vaker voor een gezonde leefstijl kiezen. Bovendien waren de geneeskundestudenten minder zelfredzaam in het leveren van bepaalde vormen van voedingszorg aan patiënten. De studenten zagen een correlatie tussen zelfredzaamheid en hun houding ten aanzien van voedingszorg.

Dit hoofdstuk illustreert dat het voedingsonderwijs tijdens de medische opleiding niet toereikend is en biedt nog meer redenen waarom dit onderwijs moet worden verbeterd.

\section{Hoofdstuk 5}

$\mathrm{Na}$ het onderzoek naar de mening van studenten over hun huidige voedingsonderwijs tijdens de medische opleiding en hun competenties en zelfredzaamheid op het gebied van voedingszorg, gaan we in Hoofdstuk 5 in op het oordeel van artsen over het voedingsonderwijs tijdens hun opleiding, en kijken we ook naar de mate waarin zij voedingszorg bieden tijdens hun werk. We hebben met name gekeken naar de mate waarin artsen voedingszorg leveren tijdens hun werk, naar hun competenties op het gebied van voe- 
dingszorg en naar belemmerende factoren. Daarnaast hebben we onderzocht in hoeverre artsen tevreden zijn over de kwaliteit van hun voedingsonderwijs en hebben we gekeken welke trainingen en informatiematerialen zij tot hun beschikking hebben voor nascholing op het gebied van voeding, en hoe effectief die zijn.

De artsen gaven aan zich gemiddeld comfortabel te voelen tijdens het bespreken voedingsgerelateerde onderwerpen met patiënten. Zij hadden echter meer affiniteit met het bespreken van voeding voor moeders en kinderen dan met het bespreken van voeding in het algemeen en voeding in relatie tot chronische ziekten. Net als de geneeskundestudenten stonden de artsen positief tegenover voedingszorg, want zij (1) vonden dat voedingszorg onder hun verantwoordelijkheid valt, (2) gaven aan dat voeding een belangrijke rol speelt bij het voorkomen en behandelen van chronische ziekten en (3) verwachtten dat hun patiënten hun advies om gezonder te gaan leven zeker zouden opvolgen. De artsen concludeerden dat hun patiënten meer informatie over voeding en voedingszorg nodig hadden dan zij als arts konden bieden.

Net als de geneeskundestudenten in Hoofdstuk 4 was er een correlatie tussen de houding van artsen ten aanzien van voedingszorg en hun affiniteit met het bieden van voedingszorg. En er was een relatie tussen hun affiniteit met voedingszorg en de mate waarin zij die zorg daadwerkelijk verleenden, want de artsen die hoger scoorden op affiniteit, gaven ook aan dat ze hun patiënten vaker voedingszorg aanboden.

De artsen erkenden het belang van diëtisten/voedingsdeskundigen en waren positief over interprofessionele samenwerking. De meesten zeiden hun patiënten voor specialistische dieetzorg door te verwijzen naar diëtisten/voedingsdeskundigen of zelf bij hen te rade te gaan.

Ondanks hun positieve houding ten aanzien van voedingszorg bleek dat de mate waarin artsen deze zorg daadwerkelijk leverden bij de verwachtingen achterbleef: hun schatting van het aantal patiënten aan wie zij daadwerkelijk voedingszorg leverden, was lager dan hun schatting van het aantal patiënten dat deze zorg naar hun mening nodig had. Verder kwam naar voren dat de meeste artsen nog geen zes minuten van hun tijd besteedden aan het bespreken van voedingskwesties. Dat artsen minder vaak dan verwacht met patiënten over voeding spraken had een aantal oorzaken: tijdgebrek en een gebrek aan kennis en vaardigheden om hierover advies te geven. Het is niet verwonderlijk dat de artsen een gebrek aan kennis en vaardigheden noemden als belemmering voor het geven van voedingszorg, want meer dan de helft gaf aan onvoldoende voedingsonderwijs te hebben gehad tijdens hun opleiding en meer dan drie kwart was niet tevreden of twijfelde over de kwaliteit en kwantiteit van hun voedingsonderwijs tijdens de medische opleiding. Deze resultaten komen overeen met het oordeel van de geneeskundestudenten, zoals besproken in Hoofdstuk 3 en 4.

Hoofdstuk 5 laat zien dat de kwaliteit en kwantiteit van het voedingsonderwijs tijdens de medische opleiding essentieel is voor het verbeteren van de voedingszorg in de praktijk. Artsen die aangaven dat hun voedingsonderwijs toereikend was, of artsen die daar 
meer tevreden over waren, bleken meer affiniteit te hebben met het bespreken van voedingskwesties met patiënten. Artsen die daarentegen negatief oordeelden over hun voedingsonderwijs, bleken slechts twee minuten of minder tijd te besteden aan het geven van voedingsadvies. Deze resultaten onderstrepen het belang van beter voedingsonderwijs tijdens de medische opleiding.

Artsen blijken zich wel bewust te zijn van hun (kennis)achterstand op het gebied van voedingszorg en gaven aan hier op eigen initiatief over te lezen en meer praktijkervaring op te doen, om hun competenties op het gebied van voedingszorg te vergroten. Het is interessant om te zien dat bijna 50\% van de artsen op dit moment gebruikmaakt van informatie- en trainingsmaterialen op het gebied van voeding, bij wijze van nascholing, en dat meer dan $80 \%$ aangaf deze materialen effectief te vinden en in de toekomst te zullen gaan gebruiken, als ze daarover de beschikking zouden hebben.

We kunnen concluderen dat artsen voedingszorg een belangrijke taak vinden, maar dat ze deze zorg niet in voldoende mate aan hun patiënten kunnen leveren vanwege ontoereikend voedingsonderwijs tijdens hun opleiding, gebrek aan tijd, kennis, zelfvertrouwen en adviserende vaardigheden.

\section{Hoofdstuk 6}

In Hoofdstuk 6 bespreken we aan de hand van een kwalitatieve aanpak hoe geneeskundestudenten oordelen over de rol van artsen in de voedingszorg, kijken we welke factoren beter voedingsonderwijs in de weg staan en met welke strategieën we daar iets aan kunnen doen. De in dit hoofdstuk gepresenteerde resultaten zijn gebaseerd op half open interviews met 23 geneeskundestudenten van de University for Development Studies, School of Medicine and Health Sciences (UDS-SMHS) die zich in de klinische fase van hun opleiding bevonden. De studenten waren van mening dat artsen een ondersteunende of aanvullende rol moeten spelen in de voedingszorg, naast de voedingsdeskundige/diëtist. Verder vonden zij dat artsen in staat moeten zijn om vast te stellen welke patiënten voedingszorg nodig hebben, dat zij basale voedingszorg moeten kunnen bieden en/of de patiënt naar een diëtist/voedingsdeskundige moeten doorverwijzen voor specialistische dieetzorg. Volgens de studenten moeten artsen in staat zijn hun patiënten te wijzen op het belang van goede voeding en moeten ze ook kunnen controleren of hun patiënten op dit gebied vooruitgang boeken.

Behalve het beschrijven van de rol van de arts, gaven de studenten ook aan dat hun voedingsonderwijs tijdens de medische opleiding ontoereikend was (vergelijkbaar met de studenten uit hoofdstuk 3, 4 en 5), waardoor ze te weinig zelfvertrouwen hebben en waarschijnlijk niet in staat zijn de beschreven taken uit te voeren.

Vervolgens gaven de studenten aan welke belemmeringen effectief voedingsonderwijs tijdens de medische opleiding in de weg staan. Deze belemmeringen zijn in drie ca- 
tegorieën onder te verdelen: persoonlijk, interpersoonlijk en afhankelijk van omgevingsfactoren. Interpersoonlijke belemmeringen liggen op het niveau van de faculteit (medewerkers of curriculumplanners). Er kan bijvoorbeeld een gebrek aan kennis zijn over klinische voeding, een gebrek aan motivatie en belangstelling voor het geven van voedingsonderwijs of curriculumplanners en faculteitsmedewerkers kunnen van mening zijn dat voedingszorg niet tot de taken van de arts behoren. Zoals de studenten aangaven, hebben interpersoonlijke belemmeringen te maken met de slechte samenwerking tussen docenten en voedingsdeskundigen/diëtisten, die beter voedingsonderwijs in de weg staat. De belemmerende omgevingsfactoren hadden te maken met de setting, het beleid en de organisatiestructuur van de medische opleidingen, bijvoorbeeld: een slechte verankering van voedingsonderwijs in het curriculum, tijdgebrek, gebrek aan docenten en te weinig ruimte in het toch al overvolle curriculum. Ook al gaat het hier om geneeskundestudenten die nog in opleiding zijn, de belemmeringen die zij noemen zijn van belang en uniek. Sommige voorbeelden komen overeen met wat geneeskundedocenten, onderzoekers op het gebied van gezondheidszorgonderwijs en artsen in eerder onderzoek hebben aangegeven.

De studenten noemden niet alleen de belemmeringen maar kwamen ook met strategieën om de belemmeringen voor effectief voedingsonderwijs weg te nemen of tot een minimum te reduceren. Voorbeelden van dergelijke strategieën waren: voeding in een vroeg stadium als thema in het (hele) curriculum inbouwen, meer bewustwording op de faculteit (docenten en curriculumplanners) dat artsen een rol moeten spelen in het leveren van voedingszorg, het curriculum doorlichten om te zien hoe voedingsleer in het onderwijs kan worden geïntegreerd en voor het onderwijs samenwerking zoeken met dietisten/voedingsdeskundigen.

De strategieën die deze geneeskundestudenten noemden zijn vergelijkbaar met die uit eerder onderzoek. Daarmee zou een deel van het probleem kunnen worden verholpen.

Deze resultaten laten zien dat geneeskundestudenten een belangrijke informatiebron kunnen zijn, die kan worden benut om het voedingsonderwijs tijdens de medische opleiding te verbeteren. De belangrijkste conclusie die we uit dit hoofdstuk kunnen trekken is dat er een aantal belemmerende factoren zijn voor het geven van goed voedingsonderwijs en dat de situatie kan worden verbeterd als de faculteit, curriculumplanners en relevante belanghebbenden voedingsonderwijs serieus nemen en beseffen dat voedingszorg een belangrijke taak van de arts is.

\section{Hoofdstuk 7 en 8}

Deze twee hoofdstukken liggen in elkaars verlengde: hoofdstuk 7 is een opstapje naar hoofdstuk 8. Nu we de huidige stand van zaken van het voedingsonderwijs hebben onderzocht, hebben gekeken naar de competenties van geneeskundestudenten en artsen 
op het gebied van voedingszorg en hebben vastgesteld welke factoren effectief voedingsonderwijs in de weg staan, bespreken we in Hoofdstuk 7 en 8 de literatuur en onderzoeken we de kenmerken van onderwijskundige interventies die zijn ontwikkeld om het voedingsonderwijs en de voedingszorg te verbeteren.

In Hoofdstuk 7 presenteren we het protocol (methoden, procedures en processen) dat we hebben gebruikt voor het beoordelen van de literatuur. Het onderzoek volgt een realistische aanpak (realistische synthese) die erop gericht is vast te stellen binnen welke context interventies worden gedaan, volgens welke mechanismen en hoe daarmee resultaten worden gegenereerd. Deze aanpak is relatief nieuw in het gezondheidszorgonderwijs en voedingsonderzoek en het protocol dient als leidraad voor toekomstige onderzoeken waarin voor deze realistische benadering wordt gekozen.

In Hoofdstuk 8 wordt vervolgens het literatuuronderzoek zelf besproken aan de hand van de volgende vraag: hoe kunnen onderwijskundige interventies ervoor zorgen dat artsen en andere zorgverleners meer en betere voedingszorg gaan leveren, en voor wie, waarom en in welke omstandigheden is dit zinvol? Uit dit onderzoek kwam naar voren dat, wil je met interventies het gewenste resultaat bereiken, het belangrijk is om eerst een behoefteanalyse uit te voeren om in kaart te brengen welke kennis of praktische ervaring studenten missen en hoe zij het beste leren. Op basis daarvan kunnen de inhoud, vorm en opzet van de curricula worden vormgegeven.

Als we de voedingszorg willen verbeteren, is het essentieel dat onderzoekers interventies ontwikkelen die leiden tot meer vaardigheden en een andere houding en minder de nadruk leggen op cognitieve aspecten, zoals kennis. Uit het onderzoek kwam naar voren dat meer kennis niet per se tot een betere voedingszorg leidt. Meer vaardigheden, een positievere houding en een grotere zelfredzaamheid lijken eerder een gedragsverandering op te leveren. Toekomstige interventies moeten dus gericht zijn op onderwijs- en leerstrategieën waarmee dat kan worden bereikt.

De resultaten van Hoofdstuk 8 laten zien dat rolmodellen en leidinggevenden die het goede voorbeeld gaven in de ziekenhuisomgeving bijdroegen aan een betere voedingszorg. De deelnemers aan het onderzoek gaven aan dat de betrokkenheid van hun leidinggevenden bij voedingszorg hun meer zelfvertrouwen gaf en dat ze zich meer geaccepteerd en geloofwaardiger voelden. Zij durfden erop te vertrouwen dat hun handelingen gewaardeerd zouden worden, waardoor ze hun gedrag op het gebied van voedingszorg aanpasten.

Daarnaast bleek dat interventies vooral tot verandering leidden als ze gericht waren op het wegnemen van structurele, systeemgebonden obstakels die het leveren van voedingszorg in de weg stonden. Voorbeelden van interventiestrategieën: een aangepaste kantooromgeving die beter aansluit op het leveren van voedingszorg, een omgeving creeren waarin artsen hun kennis op het gebied van voeding beter kunnen inzetten, het ontwikkelen van informatiemateriaal over voeding, dat drukke artsen aan patiënten kunnen meegeven, en een betere samenwerking tussen het onderwijs en de zorgverlening. 
Uit ons onderzoek bleek dat als dokters de beschikking hebben over lokaal bruikbare en praktisch relevante instrumenten en informatiematerialen over voeding en gezonde eetgewoonten, zij daardoor gestimuleerd worden om voedingszorg te leveren aan hun patiënten. Bij dit soort interventies gaat het erom dat voedingsinformatie beknopt en in eenvoudige taal wordt gepresenteerd, zodat patiënten deze goed kunnen begrijpen. Soms werd gebruikgemaakt van makkelijk te onthouden slogans, eenvoudige instructies voor thuis, op de patiënt afgestemde voedingsinformatie en lokaal relevante voorbeelden. Dankzij deze interventies konden de zorgverleners met patiënten over specifieke, persoonlijke kwesties praten in plaats van over voeding in het algemeen, en leverden ze adviezen en aanbevelingen die de patiënten relevant vonden.

In Hoofdstuk 8 laten we bovendien zien dat gezonde (eet)gewoonten van de arts ook een belangrijke rol spelen bij het leveren van voedingszorg. Interventies die artsen en andere zorgverleners stimuleerden om zelf gezonder te gaan eten, leidden er ook toe dat ze betere voedingszorg leverden. Artsen die een gezonder eetpatroon ontwikkelden, hadden meer zelfvertrouwen om patiënten over voeding te adviseren, omdat ze zichzelf als rolmodel zagen.

De informatie- en communicatietechnologie (ICT) biedt veel mogelijkheden om het voedingsonderwijs tijdens de medische opleiding en in de praktijk te verbeteren (via computers en online lesmateriaal). Computerondersteund onderwijs heeft het voordeel dat de inhoud van het lesmateriaal gemakkelijk kan worden bijgewerkt, dat studenten er zelfstandig, op een door hen gekozen moment mee kunnen werken en dat de inhoud consistent wordt gepresenteerd en toegankelijk en interactief is. Deze interventies waren ook handig voor de deelnemers omdat ze daar zelfstandig en in hun eigen tempo mee aan de slag konden. Op ICT gebaseerde interventies leidden tot een grotere voedingskennis, een positievere houding en een betere voedingszorg door de deelnemers.

Andere kenmerken van succesvolle onderwijskundige interventies met vergelijkbare positieve resultaten waren: gebruik van innovatieve les- en leermethoden en het vergroten van de zelfredzaamheid van deelnemers in het leveren van voedingszorg.

Onze realistische synthese is theoretisch van aard en het onderzoek was gebaseerd op een theorie die later is herzien op basis van de resultaten die tijdens het onderzoek naar voren kwamen. Een belangrijke herziening van de oorspronkelijke theorie was dat het begrip 'resultaat' van interventies moet worden opgesplitst in resultaten op de korte, middellange en lange termijn. De oorspronkelijke theorie hield geen rekening met deze hiërarchie, maar was slechts gericht op één resultaat, namelijk het leveren van voedingszorg. Het onderzoek liet echter zien dat het leveren van voedingszorg een resultaat op de middellange termijn is, en afhankelijk is van het bereiken van resultaten op de korte termijn, zoals meer kennis, een positievere houding, een grotere zelfredzaamheid of meer zelfvertrouwen. Het langetermijnresultaat is een beter klinisch resultaat voor patiënten, ofwel gezondere patiënten, wat kan worden bereikt met veranderingen in de voedingszorg, zoals het aanpassen van de omstandigheden, herziening van het zorgstelsel, het 
wegnemen van structurele en systeemgebonden belemmeringen, een gunstig, op voedingszorg gericht beleid, meer investeringen in preventieve zorg en het creëren van een kantooromgeving die meer geschikt is om voedingszorg te leveren.

We kunnen dus concluderen dat onderwijskundige interventies verder moeten gaan dan alleen kennisvergaring. We moeten streven naar een positievere houding van de betrokkenen en naar het vergroten van hun vaardigheden en zelfredzaamheid. Het onderzoek biedt belangrijke inzichten in welke gebieden zich lenen voor interventies en laat ook zien welke les- en leermethoden geschikt kunnen zijn om het voedingsonderwijs en de voedingszorg te verbeteren.

\section{Hoofdstuk 9}

In dit laatste hoofdstuk bespreken we de onderzoeksvragen die aan dit onderzoeksproject ten grondslag lagen en koppelen we onze bevindingen aan de relevante literatuur, trekken we conclusies en doen we aanbevelingen voor verder onderzoek. Daarnaast gaan we in op de sterkte punten en beperkingen van het onderzoek.

Het antwoord op onderzoeksvraag 1 komt erop neer dat geneeskundestudenten vonden dat voedingszorg een belangrijke rol speelt in de gezondheidszorg en bij ziekten. Daarnaast onderstreepten ze het belang van goed voedingsonderwijs. De studenten noemden een aantal belangrijke redenen waarom artsen een duidelijke rol moeten spelen in de voedingszorg: artsen hebben bijvoorbeeld een spilfunctie in het Ghanese zorgsysteem en fungeren binnen de gezondheidszorg ook als poortwachters.

Bij de beantwoording van onderzoeksvraag 2 gaven zowel de geneeskundestudenten als de artsen aan dat zij niet voldoende waren voorbereid op het leveren van voedingszorg. Ondanks dat zij er positief tegenover stonden, vonden zij dat hun kennis van voeding ontoereikend was. Zij vonden zichzelf niet zelfredzaam en het ontbrak hen aan voldoende zelfvertrouwen om voedingszorg te leveren aan hun patiënten. Om deze resultaten nog beter te kunnen onderbouwen is echter meer onderzoek nodig in andere delen van Ghana en Sub-Saharaans Afrika.

Bij de beantwoording van onderzoeksvraag 3 gaven de artsen aan dat zij niet aan alle patiënten die dat naar hun mening nodig hadden voedingszorg konden leveren vanwege een aantal belemmeringen, zoals ontoereikende kennis, tijdgebrek en onvoldoende adviserende vaardigheden. De kwaliteit en kwantiteit van het door artsen en geneeskundestudenten genoten voedingsonderwijs bepaalt in belangrijke mate hun bereidheid tot het leveren van voedingszorg en de mate waarin zij dit daadwerkelijk doen in de dagelijkse praktijk. Toekomstig onderzoek zou hier nog meer licht op kunnen werpen.

Bij het beantwoorden van onderzoeksvraag 4 kwamen de belemmeringen aan de orde die effectief voedingsonderwijs tijdens de medische opleiding in de weg staan. De geneeskundestudenten noemden een aantal belangrijke obstakels, waaronder een nu al 
overvol curriculum, gebrek aan prioriteit en een slechte samenwerking tussen docenten en diëtisten/voedingsdeskundigen.

De antwoorden op onderzoeksvraag 5 lieten zien dat zowel de geneeskundestudenten als de artsen aan zelfstudie doen om hun competenties en zelfredzaamheid op het gebied van voedingszorg te vergroten, omdat beide groepen van oordeel waren dat hun voedingsonderwijs tijdens de medische opleiding ontoereikend was. Uit het onderzoek kwam ook de bereidheid van artsen naar voren om hun kennis over voeding te vergroten.

De onderzoeksvragen 6 en 7 gaan over het gebruik van de realistische synthese in het literatuuronderzoek om te achterhalen wat de kenmerken zijn van succesvolle onderwijskundige interventies die gericht zijn op verbetering van de competenties op het gebied van voedingszorg en de voedingszorg in de praktijk. Het protocol van deze realistische synthese dient als richtlijn voor de opzet van vergelijkbare onderzoeken in het gezondheidszorgonderwijs en voedingsonderzoek.

We moeten concluderen dat de in dit proefschrift gepresenteerde resultaten hun beperkingen hebben omdat het een cross-sectioneel onderzoek betreft waarbij gebruik werd gemaakt van enquêtes met open vragen en omdat het onderzoek grotendeels bij één en dezelfde instelling is uitgevoerd. Toch bieden de resultaten belangrijke aanknopingspunten voor het medisch onderwijs. Onze bevindingen dienen als basis voor de herziening van en innovaties in het medisch curriculum in Ghana. Het voedingsonderwijs verdient meer aandacht, want uit ons onderzoek komt duidelijk naar voren dat dit momenteel ontoereikend is. Daarnaast moet de rol van artsen worden vastgesteld en aangepast. Voeding moet een belangrijk thema worden binnen het complete medische curriculum. Dat is een belangrijke voorwaarde voor het verbeteren van het voedingsonderwijs. 

Valorisation 


\section{Introduction}

Nevertheless, the findings of this thesis have important insights and consequences for the teaching and learning of nutrition care in undergraduate medical education and also the provision of nutrition care in the hospital setting.

\section{Relevance}

In this doctoral thesis we have undertaken a needs assessment of the situation of nutrition care and its education in the medical curriculum. The research has answered the general research question posed at the beginning i.e. what is the current practice of a) nutrition education for medical students and b) nutrition care of practicing doctors in Ghana? The findings bring to fore that nutrition education is inadequate in the medical curriculum in Ghana and that nutrition care is below the needs of patients. The research presented in this thesis also evaluated the perspectives of two important stakeholders of medical education i.e. medical students in training and doctors in practice. This approach granted us the opportunity to have a holistic and a comprehensive needs assessment of the situation of nutrition education in the curriculum. This resulted in our finding that both students and practicing doctors alike value nutrition education and consider it to be relevant and important to their training and practice as medical doctors. In addition, both have demonstrated their readiness to learn more about nutrition to cater for their inadequacies during their training.

We recognise that, the inadequacy in nutrition education in the medical curriculum is an age-old concern globally and also equally recognise efforts made elsewhere to help improve nutrition education in medical training. We thus suggest the need for all stakeholders, including the Ghana National Accreditation Board and the Medical and Dental Council of Ghana, to collaborate to help improve medical nutrition education in Ghana. Other stakeholders such as deans of medical schools, faculty, and students, the Ministry of Health, the Ghana Health Service, and the Ghana Medical Association should also be included in such a collaboration to help improve nutrition education. As have been done elsewhere the regulatory bodies (i.e. the National Accreditation Board and the Ghana Medical and Dental Council) should come out with nutrition syllabi for medical training and should provide a required number of hours for nutrition education for all medical schools in the country.

We have shown in this doctoral thesis that the nutrition knowledge of medical students vary by their level of training and tend to wane as they progress. Without reinforcement clinical students may not retain the nutrition knowledge they acquired during their pre-clinical training. These findings demonstrate the need for nutrition education to be applied throughout the entire medical curriculum. This will allow for reinforcement and application of nutrition knowledge learnt during pre-clinical education. This should be informed by a review of the entire curriculum to identify areas for the integration of nu- 
trition content in the curriculum. Given the fact that the medical curriculum is overcrowded, integration of nutrition content through the entire curriculum may be an important way of improving nutrition education, allowing the acquisition of more nutrition content without increasing the contact hours of the entire medical curriculum.

In this doctoral thesis we have exhibited that the workplace setting is an important training/learning resource for nutrition care. Adding to the literature on workplace learning, practicing doctors have found their clinical practice as an important contributor to their current nutrition care competencies. In addition we also found that the modelling of nutrition care by superiors such as consultants, specialist, etc may also result in improved nutrition practice behaviour. A workplace culture of nutrition care provides a conducive environment for doctors to include nutrition care in their consultations with patients. We thus suggest that consultants, specialists and clinical teachers should model nutrition care, thereby encouraging junior doctors and medical students to have the confidence and the support to provide nutrition care in the practice setting.

Self-efficacy in nutrition care and attitudes towards nutrition care have been shown to be important determinants of nutrition practice and referral behaviour among practicing doctors. Among medical students, self-efficacy and attitudes towards nutrition care correlated in that those who felt self-efficacious were more likely to have positive attitudes towards nutrition care. As reported in our realist review of the literature of educational interventions to improve nutrition care practice among doctors, self-efficacy in nutrition care was found to result in improved nutrition practice behaviour. It was found that it is more important to build doctors' attitude and empathy for nutrition care than increasing their knowledge only. These findings provide important information for curriculum planners and designers of nutrition care educational intervention to prioritise nutrition care self-efficacy and attitudes towards nutrition care in their design of such interventions. They should thus adopt teaching and learning strategies that will help students and doctors alike to acquire appropriate skills, develop positive attitudes and build selfefficacy/confidence in nutrition care.

Having considered their nutrition education during medical training to be inadequate, practicing doctors considered a number of continuing education in nutrition training and learning resources to be effective and demonstrated their willingness to adopt them in the future if made available. These included short nutrition seminars or lectures; online nutrition courses designed for doctors; dietician/nutritionist facilitated workshops for doctors; continuing medical education conferences on nutrition with continuing professional development (CPD) credits for participation; making access to nutrition-related journal articles; nutrition newsletters and nutrition information on patient handouts. These findings demonstrate their high interest and a favourable attitude towards improving their current nutrition care competencies. It is thus imperative for medical educators, nutrition care professionals and researchers to design and make available to doctors such continuing nutrition education programmes. The availability of these programmes will 
provide avenues for doctors to learn more about nutrition during practice. Medical licensing bodies, such as the Ghana Medical and Dental Council, should assign continuing professional development (CPD) credits to nutrition-related continuing education programmes in a bit to encourage doctors to participate in such programmes.

Having undertaken a comprehensive needs assessment of nutrition education, barriers and nutrition care we undertook a review of the literature to identify appropriate interventions that can be designed to help improve nutrition education and nutrition care practice. Following a realist approach (a theory-driven, non-traditional systematic review) the review brought to bear the characteristics of educational interventions that work to improve nutrition care and delivery as well as those that do not work. The findings have increased our understanding of the context within which educational interventions can improve nutrition care competence and practice, and the mechanisms through which these educational interventions work and the kind of outcomes that can be generated through those educational interventions. In addition these findings are transferrable across settings to better support nutrition education to improve nutrition care competence and practice of nutrition care. The realist approach also showed that educational interventions to improve nutrition care competence and delivery are complex and do not operate in a linear fashion. Designers of such educational interventions should thus be aware of their complex nature which should be highlighted during planning, designing and implementation stages of such interventions.

The review also resulted in the development of a conceptual model of how educational interventions operate to bring about outcomes. It is a framework of the conditions, circumstances, mechanisms and context within which educational interventions work to bring about short-and long-term outcomes. This finding provides a framework that will inform the design of future educational interventions to improve nutrition care competencies and delivery. It will help designers of educational interventions to map out the essential components of such interventions in order to benchmark their effectiveness.

\section{Target groups}

The research presented in this doctoral thesis serves as a resource for varied target groups. They include: curriculum planners and managers, researchers in health professions education and nutrition, medical students, doctors in practice, faculty (clinical and pre-clinical teachers), and nutritionists, dieticians, and other health professionals.

\section{Curriculum planners and managers}

In this thesis medical students identified a number of barriers that did not allow for effective nutrition education. Paramount among these was the lack of priority for nutrition education. For nutrition education to gain priority in medical education, it is important for nutrition education to be established and recognized as a discipline within the medical 
curriculum that will enable faculty to specialize in nutrition. Curriculum planners and managers are pivotal in this process.

\section{Researchers in health professions education and nutrition}

The research presented in this thesis sets the background for researchers in health professions education and nutrition to conduct further research in nutrition education and to come out with interventions that will improve nutrition care and its delivery. A number of future research directions and ideas have been presented in this thesis that can be utilized by researchers in health professions education and nutrition to bring about evidence that will contribute new knowledge to the literature on nutrition education.

\section{Medical students}

Four studies presented in this studies were conducted among medical students. Two were quantitative cross-sectional studies and the other two were qualitative. The quantitative studies investigated students' nutrition care competencies, self-efficacy, quality and quantity of their nutrition education, and their preparedness to provide nutrition care in the general practice setting. The findings of these studies brought to the fore the inadequacy of nutrition education in the medical curriculum resulting in medical students feeling inadequately prepared, less competent and self-efficacious to provide nutrition care. It also demonstrated students' willingness and interest to learn more about nutrition. The qualitative studies explored the views, opinions and perspectives of medical students regarding the role of nutrition education in their training as well as their perception of the roles and responsibilities of doctors in the provision of nutrition care. Furthermore they explored students' views, opinions and perspectives regarding barriers to effective nutrition education and suggested strategies to overcome such barriers. The findings of these studies have shown that medical students are important stakeholders of the curriculum and can make important contributions towards improving their curriculum and subsequently the learning environment. It thus implies that students should be involved in any efforts that are geared towards planning, review and implementation of the curricula in health professions education. Furthermore, understanding the opinions of medical students regarding the role of nutrition care may help to gauge their attitudes and the acceptability of nutrition education. Our findings also serve as a proxy for medical students' readiness to embrace educational interventions to improve nutrition education, nutrition care competence and practice when they become medical doctors. Importantly, they recognise the role of other members of the multidisciplinary healthcare team, such as dieticians and/or nutritionists, thereby encouraging inter-professional healthcare. 


\section{Doctors in practice}

One study in this thesis evaluated practicing doctors' nutrition care practices, barriers, competencies and education in nutrition. Findings from that study showed that doctors' provision of nutrition care in the general practice setting does not meet the needs of patients. Doctors reportedly felt that the quantity and quality of nutrition care they provide fall short of the nutrition needs of their patients. They are simply unable to meet the needs of the patients regarding nutrition care. It adds to the literature that doctors recognise their inability to meet the nutrition care needs of their patients and have also demonstrated that they require further training in nutrition. These are favourable attitudes that can inform the design of future educational interventions to improve nutrition care competencies and delivery.

\section{Faculty (clinical and pre-clinical teachers)}

The findings of a qualitative study of medical students' perception of barriers to effective nutrition education identified unavailability of faculty to teach nutrition as an important barrier. The role of the teacher in nutrition education and nutrition care practice is paramount. Faculty development programmes on nutrition should thus be developed. Furthermore, faculty should demonstrate to students the important role of nutrition care and create learning environments that promote the acquisition of nutrition counselling skills and the development of favourable attitudes.

\section{Nutritionists, dieticians, and other health professionals}

A sense of inter-professionalism was demonstrated by both medical students and practicing doctors. Being aware of their limitations regarding nutrition care and also recognising the important role of the nutritionist/dieticians to patient care, doctors reportedly referred patients to nutritionists/dietician for specialist dietary care. Again both medical students and practicing doctors alike considered nutritionists/dieticians as important nutrition training/learning resources and preferred them to facilitate nutrition training workshops. No wonder students suggested faculty to collaborate with the nutritionists/dieticians in their training. These findings demonstrate the need for collaboration among faculty, clinical educators, nutritionists/dieticians and other healthcare professionals in efforts that are geared towards improving nutrition education and care. There is thus the need for multidisciplinary collaboration at all levels of curriculum design, teaching, learning and clinical practice. In addition there is the need for medical doctors, nutritionists, dieticians and other healthcare professionals to come together and begin to discuss, identify and align the roles and responsibilities of the doctors and other healthcare professionals regarding the provision of nutrition care. 


\section{Activities}

As an immediate activity, the results of this thesis will be presented to the faculty of the University for Development Studies, School of Medicine and Health Sciences (UDS-SMHS) for them to appreciate the situation of nutrition education in the curriculum and also deliberate on how best nutrition education can be incorporated into the curriculum. The research presented in this thesis consists of empirical studies undertaken in the context of a single undergraduate medical programme and among practicing doctors in Ghana. We will thus explore the generalizability of our findings in other health care programmes, both at home and internationally. A number of the studies have been presented at international conferences on medical and nutrition education. In addition, all studies presented in this thesis except one have been published in journals of varied disciplines including health professions education, nutrition, medical and public health. 

Acknowledgement 
First of all I will like to thank the Almighty God for His continuous guidance, protection and blessings all these years. I am very grateful for His unfailing favour upon my life.

I am immensely grateful to Prof. Albert JJA Scherpbier, my lead supervisor, for his motivation, focused comments, foresight, patience and vast knowledge. His guidance has helped me in the research and writing of this thesis. I am also grateful to the other members of the supervisory team, Dr. Fred Stevens and Dr. Paul Aryee, for their insightful comments, directions, ideas and general support to come out with this piece of work.

I am very much appreciative of Prof. Francis A Abantanga, Dean, School of Medicine and Health Sciences, University for Development Studies (SMHS-UDS) for his continued support and encouragement in my career progression.

I am very grateful to Dr. Edward Gyader and Prof. Kyei-Aboagye (now late), past Deans of SMHS-UDS, for the different roles they played in creating an enabling environment for me to carry out my PhD project.

I will like to immensely thank the Netherlands Initiative for Capacity Development in Higher Education (NICHE) for funding my PhD by means of paying my fees and helping me attend conferences and to meet my supervisors. I am grateful to all the team at SHE Collaborates of the FHML especially Geraldine, Emmaline, and Hennie for all the administrative support whenever I have to travel to attend international conferences, as well as for their continued interest and monitoring of the progress of my PhD. I will also like to make special mention of Lilian Swaen for her administrative support in helping me obtain some letters relating to my PhD.

I am grateful to Mr. Anthony Amalba, my colleague PhD candidate and Head of Department for the Department of Health Professions Education and Innovative Learning, for granting me the opportunity to do the $\mathrm{PhD}$ and for the stimulating discussions that we have had and will continue to have in the future.

I will like to thank my wife, Patience, for the love, care and always edging me on to work harder in order to realize my dreams. I am grateful to her for spending extra time and energy to take care of the kids while I worked on my PhD project and allowing me to work late into the night to enable me meet deadlines to submit manuscripts. I will also like to thank my two sons, Bangya and Naamkulna, for the little fun distractions they have been giving me whenever I sit on my computer to work at home. These were motivating and encouraging. I dedicate this work to them and my wife.

Finally, I will like to thank my dad, the Chief of Tindongo, NAAB MELUGSUNG KUPASAAH MOGRE I, and my mums, Lamisi, Doris, Lahiri and Comfort, for making it possible for me to earn an education and to earn it to the terminal level of a PhD. I will also like to thank my siblings for their continued interest in my career progression. 
Curriculum Vitae 
Victor was born on $27^{\text {th }}$ July 1982 in Bolgatanga, Ghana. He spent his childhood and youthful years in Bolgatanga, were he obtained his secondary level of education at the Bolgatanga Secondary School (BIGBOSS). In 2002, he gained admission into the University for Development Studies (UDS) to pursue a bachelor's degree in Community Nutrition. He graduated with a First Class in 2006. In April 2010, he obtained a master's degree in health Professions Education from the Joint Masters in Health Professions Education programme provided by Maastricht University in the Netherlands and Suez Canal University in Egypt.

After obtaining his bachelor's degree, Victor worked as a teaching assistant the School of Medicine and Health Sciences of the University for Development Studies and subsequently got promoted to a Senior Research Assistant the following year. For a period of 10 years, Victor rose through the ranks from Assistant Lecturer in 2010 to Lecturer in 2013 to his current position of Senior Lecturer in 2017 in the Department of Health Professions Education and Innovative Learning of the University for Development Studies. In his current position, Victor facilitates staff development workshops in topics relating to how to conduct PBL tutorials, interactive lectures and assessments. He also participates in curriculum review and development processes as well as coordinates teaching and learning activities at the School of Medicine and Health Sciences. In addition, Victor teaches nutrition to medical students. While at his work in the University, Victor has attended short courses in nutrition, quantitative and qualitative research in health professions education and academic programme review. Victor has several publications in peerreviewed journals and has peer reviewed articles for a number of journals, including BMC Medical Education, BMJ Open and Plos one.

From 2014 to 2018, Victor has been working on his PhD project, described in this thesis at the School of Medicine and Health Sciences of the University for Development Studies. During this period, Victor participated and presented abstracts in a number of local and international conferences, including the 2017 Annual Interdisciplinary Conference at the University for Development Studies, Ghana; the International Summit on Nutrition Education at the University of Cambridge in the UK; the Ghana Biomedical Convention at the University of Ghana; the Rogano conference at the University of Glasgow and the First International Conference on Realist Approaches to Evaluation and Synthesis at the University of Liverpool.

Victor's research interests include health professions education, nutrition, self-care behaviours, diabetes and obesity. In the future, Victor will continue to teach, mentor students, develop courses and conduct research in the School of Medicine and Health Sciences. His future research focus in health professions education will include nutrition education in other health professions, such as nursing and dentistry, as well as the design and implementation of interventional studies to improve nutrition education.

In November 2012, Victor married his wife, Patience, and they live together in Tamale, Ghana with their two sons, Bangya and Naamkulna (born in 2014 and 2017, respectively). They enjoy taking early morning walks together, going on holidays and having family time with their two sons. 


\section{List of Publications}


Bibliometric data: According to Google Scholar, my publications have been cited 263 times since 2013 as at $14^{\text {th }}$ May 2018. My h-index is 10 and i10-index is 10.

1. Mogre V., Stevens, F. C., Aryee, P. A., Matorwmasen-Akkermans, F. L., Abubakari, B., \& Scherpbier, A. J. Nutrition Care Practices, Barriers, Competencies and Education in Nutrition: a Survey among Ghanaian Medical Doctors. Med Sci Educ 2018; 1-10. 2.

2. Mogre V, Stevens FC, Aryee PA, Amalba A, Scherpbier AJ. Why nutrition education is inadequate in the medical curriculum: a qualitative study of students' perspectives on barriers and strategies. BMC Med Educ 2018;18(1):26.

3. Mogre V, Stevens F, Aryee PA, Scherpbier AJ. Nutrition in Medicine: Medical Students' Satisfaction, Perceived Relevance and Preparedness for Practice. Health Prof Educ 2018;4(1):31-38.

4. Mogre V, Aryee PA, Stevens FC, Scherpbier AJA. Future Doctors' Nutrition-Related Knowledge, Attitudes and Self-Efficacy Regarding Nutrition Care in the General Practice Setting: A Cross-Sectional Survey. Med Sci Educ 2017;27(3):481-488.

5. Mogre V, Scherpbier AJ, Stevens F, Aryee P, Cherry MG, Dornan T. Realist synthesis of educational interventions to improve nutrition care competencies and delivery by doctors and other healthcare professionals. BMJ Open 2016;6(10):e010084.

6. Mogre V, Scherpbier A, Dornan T, Stevens F, Aryee PA, Cherry MG. A realist review of educational interventions to improve the delivery of nutrition care by doctors and future doctors. Systematic reviews. 2014;3(1):1.

7. Mogre V., Yakubu, A., Fuseini, M., Amalba, A., \& Aguree, S. Nurses' knowledge and attitudes regarding malnutrition in children and its management in Ghana. Curationis 2017; 40(1):1-8.

8. Amalba A, van Mook W, Mogre V, Scherpbier A. The perceived usefulness of community based education and service (COBES) regarding students' rural workplace choices. BMC Med Educ 2016;16(1):130.

9. Amalba A, van Mook WNKA, Mogre V, Scherpbier AJJA. The effect of Community Based Education and Service (COBES) on medical graduates' choice of specialty and willingness to work in rural communities in Ghana. BMC Med Educ 2016;16(1):79.

10. Mogre V, Amalba A. Psychometric properties of the dundee ready educational environment measure in a sample of Ghanaian Medical Students. Educ Health 2016;29(1):16.

11. Mogre V, Amalba A. Approaches to learning among Ghanaian students following a PBL-based medical. Educ Med J 2015;7(1).

12. Mogre $\mathrm{V}$, Amalba A. Assessing the reliability and validity of the Revised Two Factor Study Process Questionnaire (R-SPQ2F) in Ghanaian medical students. J Educ Eval Health Prof 2014;11.

13. Mogre V, Amalba A, Saaka M, Kyei-Aboagye K. Medical students' achievement on the Bachelor of Medicine, Bachelor of Surgery/Chirurgery Final Part I and II licensing examination: a comparison of students in problem-based learning, community-based 
education and service, and conventional curricula in Ghana. J Educ Eval Health Prof 2014;11.

14. Amalba A, Mogre V, Appiah MN, Mumuni WA. Awareness, use and associated factors of emergency contraceptive pills among women of reproductive age (15-49 years) in Tamale, Ghana. BMC Women's Health 2014;14(1):114.

15. Amidu N, Owiredu W, Saaka M, et al. Determinants of childhood obesity among basic school children aged 6-12 years in Tamale Metropolis. J Med Biomed Sci 2013;2(3):26-34.

16. Mogre V, Abanga ZO, Tzelepis F, Johnson NA, Paul C. Adherence to and factors associated with self-care behaviours in type 2 diabetes patients in Ghana. BMC Endocr Disord 2017;17(1):20.

17. Mogre V, Abedandi R, Salifu ZS. Distorted self-perceived weight status and underestimation of weight status in diabetes mellitus type 2 patients. PLoS One 2014;9(4):e95165.

18. Mogre V, Abedandi R, Salifu ZS. Prevalence of obesity and systemic hypertension among diabetes mellitus patients attending an out-patient diabetes clinic in a Ghanaian Teaching Hospital. Diabetes Metab Syndr 2014;8(2):67-71.

19. Mogre V, Abedandi R, Salifu ZS. Correlates and Predictors of Increasing Waist Circumference in Patients with Type 2 Diabetes Mellitus: A Cross-Sectional Study. Int Sch Res Notices 2014;2014.

20. Mogre V, Aleyira S, Nyaba R. Misperception of weight status and associated factors among undergraduate students. Obes ResClin Pract 2015;9(5):466-474.

21. Mogre V, Aleyira S, Nyaba R. Factors associated with central overweight and obesity in students attending the University for Development Studies in Tamale, Ghana: a cross-sectional study. South Afr J Clin Nutr 2014;27(2):69-74.

22. Mogre V, Aneyire E, Gyamfi E. Physical activity and BMI status of school-age children in Tamale, Northern Ghana. Pakistan J Nutr 2013;12(5):484.

23. Mogre V, Ansah GA, Marfo DN, Garti HA. Assessing nurses' knowledge levels in the nutritional management of diabetes. International Journal of Africa Nursing Sciences. 2015;3:40-43.

24. Mogre V, Apala P, Nsoh JA, Wanaba P. Adiposity, hypertension and weight management behaviours in Ghanaian type 2 diabetes mellitus patients aged 20-70 years. Diabetes Metab Syndr 2016;10(1):S79-S85.

25. Mogre V, Atibilla J, Kandoh B. Association between breakfast skipping and adiposity status among civil servants in the Tamale metropolis. J Med Biomed Sci 2013;2(3):1-7.

26. Mogre V, Dery M, Gaa PK. Knowledge, attitudes and determinants of exclusive breastfeeding practice among Ghanaian rural lactating mothers. Int Breastfeed J 2016;11(1):12.

27. Mogre V, Gaa PK, Abukari RNS. Overweight, obesity and thinness and associated factors among school-aged children (5-14 years) in Tamale, Northern Ghana. Euro Sci J 2013;9(20). 
28. Mogre V, Johnson NA, Tzelepis F, Shaw J, Paul C. Adherence to self-care behaviours and associated barriers in type 2 diabetes patients of low-and middle-income countries: a systematic review protocol. Syst Rev 2017;6(1):39.

29. Mogre V, Mwinlenaa P, Oladele J, Amalba A. Impact of physical activity levels and diet on central obesity among civil servants in Tamale metropolis. J Med Biomed Sci 2012;1(2).

20. Mogre V, Mwinlenna PP, Oladele J. Distorted self-perceived weight status and its associated factors among civil servants in Tamale, Ghana: a cross-sectional study. Arch Public Health 2013;71(1):30.

31. Mogre V, Nsoh JA, Wanaba P, Apala P. Demographic factors, weight management behaviours, receipt of healthcare professional's counselling and having knowledge in basic anthropometric measurements associated with underassessment of weight status in overweight and obese type 2 diabetes patients. Obes Res Clin Pract 2016;10(4):381-389.

32. Mogre V, Nyaba R, Aleyira S. Lifestyle risk factors of general and abdominal obesity in students of the school of medicine and health science of the University of Development Studies, Tamale, Ghana. Int Sch Res Notices Obes 2014;2014.

33. Mogre V, Nyaba R, Aleyira S, Sam NB. Demographic, dietary and physical activity predictors of general and abdominal obesity among university students: a crosssectional study. SpringerPlus 2015;4(1):226.

34. Mogre V, Salifu ZS, Abedandi R. Prevalence, components and associated demographic and lifestyle factors of the metabolic syndrome in type 2 diabetes mellitus. J Diabetes Metab Disord 2014;13(1):80.

35. Mogre V, Wanaba P, Apala P, Nsoh JA. Self-reported receipt of healthcare professional's weight management counselling is associated with self-reported weight management behaviours of type 2 diabetes mellitus patients. SpringerPlus 2016;5(1):379.

36. Mogre V, Yakubu A, Fuseini M, Amalba A, Aguree S. Nurses' knowledge and attitudes regarding malnutrition in children and its management in Ghana. Curationis 2017;40(1):8 pages.

37. Opare-Asamoah K, Majeed S, Quaye L, et al. Assessing the prevalence of hypertension and obesity among diabetics in the Tamale Metropolis, Ghana. Br J Med Med Res 2017;2 
